Supporting Information

\title{
Robust titanium phenolate-modified microspheres as reusable affinity material for selectively capturing phosphopeptides from complicated biosamples
}

\author{
Shujuan $\mathrm{Ma}^{\mathrm{a}}$, Ruizhi Tang ${ }^{\mathrm{a}}$, Yang Yu ${ }^{a, c}$, Luwei Zhang ${ }^{a}$, Haiyang Zhang ${ }^{a}$, \\ Wenyong Ding ${ }^{b *}$, Junjie $\mathrm{Ou}^{a, c *}$
}

${ }^{a}$ Key Laboratory of Separation Science for Analytical Chemistry, Dalian Institute of Chemical Physics, Chinese Academy of Sciences (CAS), Dalian, 116023, China

${ }^{b}$ Biochemistry and Molecular Biology Department of College of Basic Medical Sciences, Dalian Medical University, Dalian 116044, China

${ }^{c}$ University of Chinese Academy of Sciences, Beijing 100049, China

To whom correspondence should be addressed:

(W. Ding)Tel:+86-411-86110312. Fax: +86-411-86110307.

E-mail: dingwy@dmu.edu.cn

(J. Ou) Tel: +86-411-84379576. Fax: +86-411-84379620.

E-mail: junjieou@dicp.ac.cn

Number of Pages: 150

Number of Figures: 5

Number of Tables: 4 
The preparation protocol of MAR microspheres

Cell culture and lysis

Tryptic digestion of proteins

Fig. S1 Enrichment protocol of phosphopeptides by IMAC strategy.

Fig. S2 Representative optical micrograph of MAR.

Fig. S3 Full XPS spectra of (a) pristine MAR, (b) ethylenediamine-modified MAR, (c) pyrogallol-modified MAR, (d) $\mathrm{Ti}^{4+}$-MAR and (e) $\mathrm{Zr}^{4+}$-MAR.

Fig. S4 Structural formula of pyridoxal 5'-phosphate.

Fig. S5 Venn diagrams of identified unique (a) phosphopeptides and (b) phosphoproteins from tryptic digest of HeLa cell proteins with commercial SPE-Ti-IMAC material as the IMAC sorbent by cLC-MS/MS.

Table S1 Kinetic parameters of pyridoxal 5-phosphate adsorption on $\mathrm{Ti}^{4+}$ - MAR by pseudo-first-order and pseudo-second-order models.

Table S2 Adsorption isotherm parameters by Langmuir and Freundlich models of pyridoxal 5-phosphate on $\mathrm{Ti}^{4+}$ - MAR.

Table S3 Enriched phosphopeptides from the tryptic digest of Hela cell proteins with $\mathrm{Ti}^{4+}-\mathrm{MAR}$ as the IMAC sorbent by cLC-MS/MS.

Table S4 Enriched phosphopeptides from the tryptic digest of Hela cell proteins with commercial SPE-Ti-IMAC material as the IMAC sorbent by cLC-MS/MS. 


\section{The preparation protocol of MAR microspheres}

First, $1.0 \mathrm{~g}$ of PVP was dissolved in $120 \mathrm{~mL}$ of water in a three-necked round-bottom flask. Second, a toluene solution containing $15 \mathrm{~mL}$ of EDMA, $20 \mathrm{~mL}$ of GMA and $0.12 \mathrm{~g}$ of BPO was added to the flask. Next, the mixture was stirred at 130 $\mathrm{rpm}$ in the protection of nitrogen stream at $70{ }^{\circ} \mathrm{C}$ for $24 \mathrm{~h}$ to form microspheres. The microspheres were then washed three times with ethanol and dried in a vacuum oven at $60^{\circ} \mathrm{C}$ to obtain polydisperse microspheres. 


\section{Cell culture and lysis}

Human cervical cancer cells (Hela cells) were cultured in RPMI 1640 medium, added $1 \%$ streptomycin/penicillin and $10 \%(\mathrm{w} \%)$ new born bovine serum at $37{ }^{\circ} \mathrm{C}$ in $5 \% \mathrm{CO}_{2}$ atmosphere. The cells were harvested when they grew to about $80 \%$ density. The resulting Hela cells were firstly rinsed twice with the cold phosphate buffered solution (PBS, $0.01 \mathrm{~mol} / \mathrm{L}$ phosphate, $0.15 \mathrm{~mol} / \mathrm{L}$ sodium chloride, $\mathrm{pH}$ 7.4). Then the dish was scraped in ice-cold lysis buffer containing 0.5 mM EGTA, 1 mM EDTA, 65 mmol/L DTT, $8 \mathrm{~mol} / \mathrm{L}$ urea, $50 \mathrm{mmol} / \mathrm{L}$ Tris- $\mathrm{HCl}$ (pH 7.4), phosphatase inhibitor, $2 \%$ protease inhibitor cocktail and $1 \%$ Triton $\mathrm{X}-100$. The scraped cell solution was transferred into a centrifuge tube and sonicated for 25 min following by centrifuging at $25,000 \mathrm{~g}$ at $4{ }^{\circ} \mathrm{C}$ for $35 \mathrm{~min}$. The collected supernatant was added five volumes of ice-cold precipitation solution (ethanol/acetone/acetic acid $=50 / 50 / 0.1, \mathrm{v} / \mathrm{v} / \mathrm{v}$ ) and placed at $-20{ }^{\circ} \mathrm{C}$ overnight for precipitating proteins. The precipitated proteins were washed with ice-cold acetone and ethanol, successively. The concentration of proteins was finally measured by BCA assay. 


\section{Tryptic digestion of proteins}

Two milligrams of proteins ( $\beta$-casein/BSA/Hela cell) were firstly dissolved in a denaturing solution containing $8.0 \mathrm{~mol} / \mathrm{L}$ urea and $50 \mathrm{mmol} / \mathrm{L}$ Tris- $\mathrm{HCl}(\mathrm{pH}, 8.2)$ in a centrifuge tube. After adding $20 \mu \mathrm{L}$ of $20 \mathrm{mmol} / \mathrm{L}$ DTT aqueous solution, the mixture was incubated at $37^{\circ} \mathrm{C}$ for $2 \mathrm{~h}$. Then, it was treated in darkness for $30 \mathrm{~min}$ at room temperature for alkylation by introducing $40 \mathrm{mmol} / \mathrm{L}$ IAA. Subsequently, the solution was diluted 8 times with $50 \mathrm{mmol} / \mathrm{L}$ Tris- $\mathrm{HCl}$ buffer and digested at $37^{\circ} \mathrm{C}$ for $16 \mathrm{~h}$ by trypsin with an enzyme-to-protein ratio of 1:25 (w/w). Finally, the resulting digest was lyophilized and stored in a $-20{ }^{\circ} \mathrm{C}$ freezer before use. 


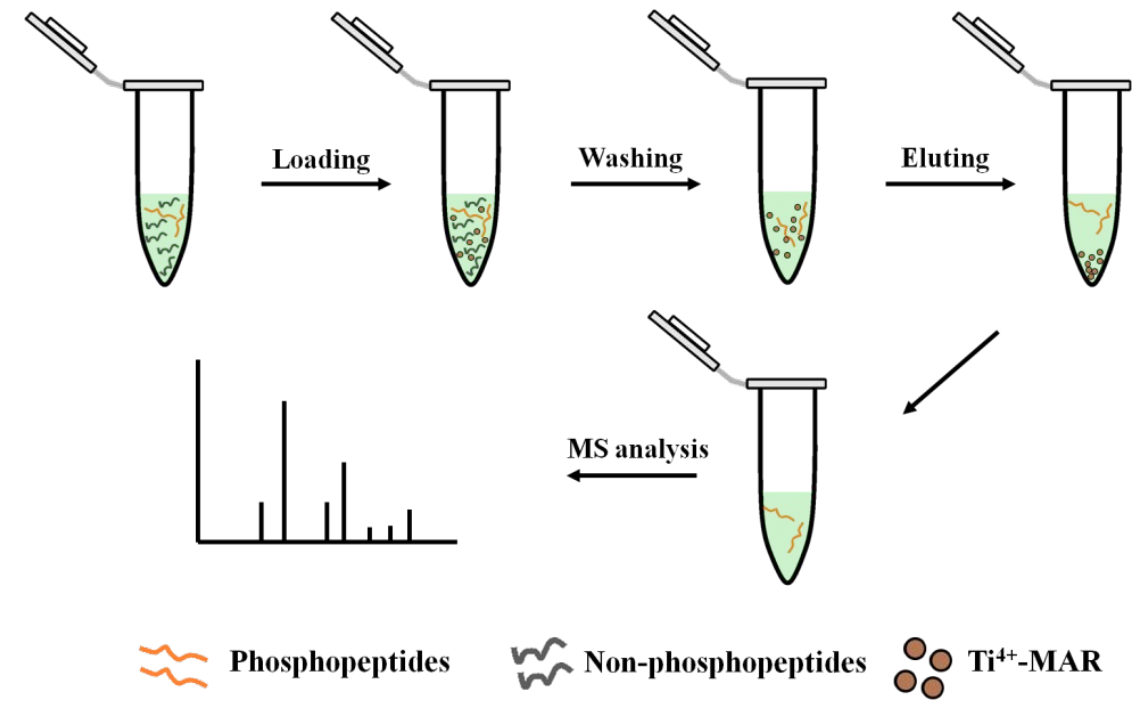

Fig. S1 Enrichment protocol of phosphopeptides by IMAC strategy. 


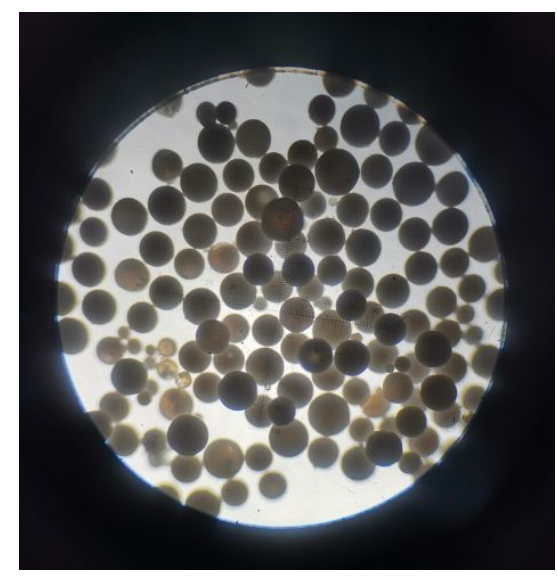

Fig. S2 Representative optical micrograph of MAR. 

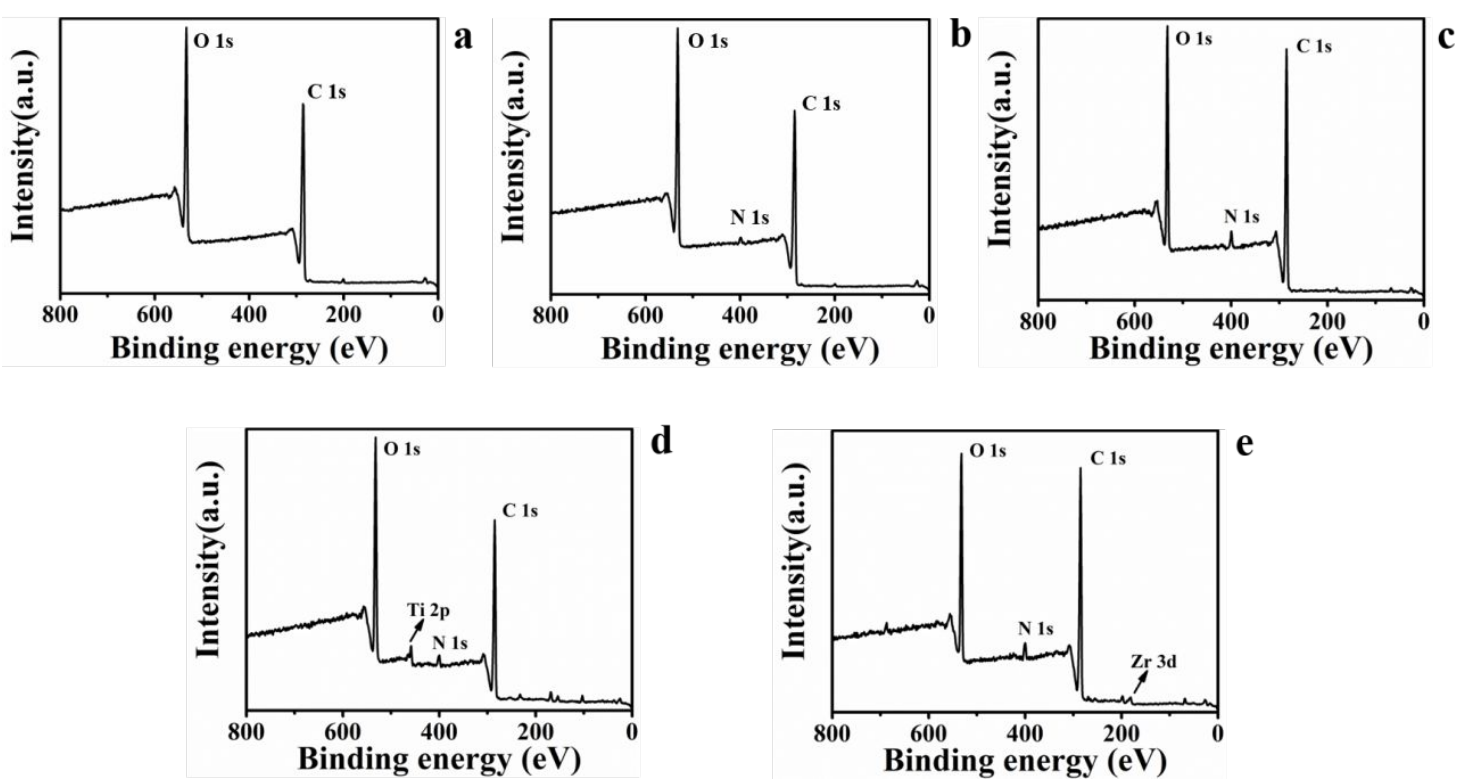

Fig. S3 Full XPS spectra of (a) pristine MAR, (b) ethylenediamine-modified MAR, (c) pyrogallol-modified MAR, (d) $\mathrm{Ti}^{4+}$-MAR and (e) $\mathrm{Zr}^{4+}$-MAR . 


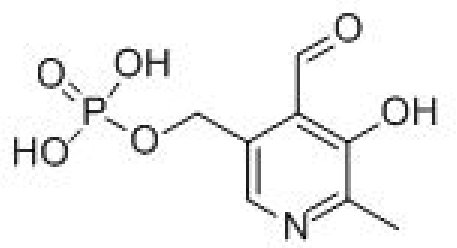

Fig. S4 Structural formula of pyridoxal 5'-phosphate. 


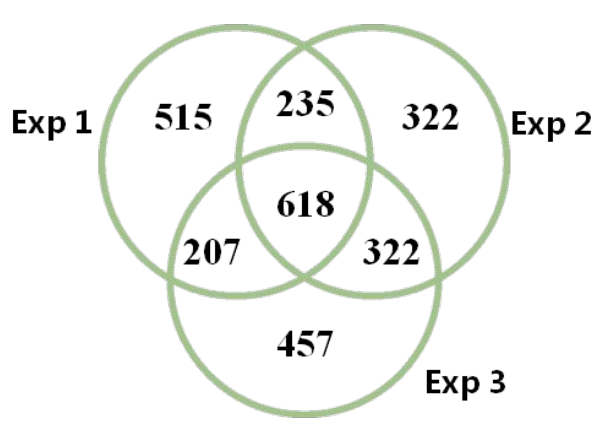

Total 2764 phosphopeptides

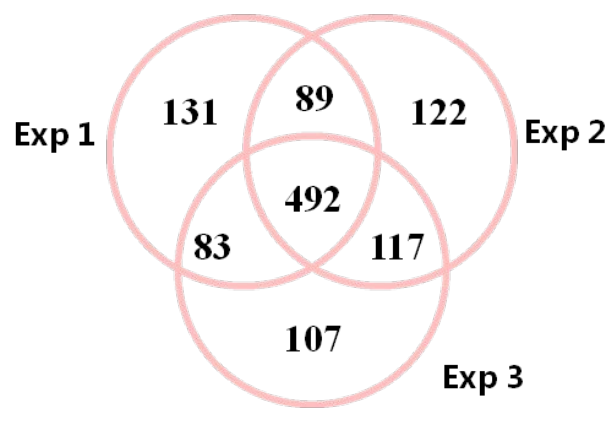

Total 1141 phosphoproteins

Fig. S5 Venn diagrams of identified unique (a) phosphopeptides and (b) phosphoproteins from tryptic digest of Hela cell proteins with commercial SPE-Ti-IMAC material as the IMAC sorbent by cLC-MS/MS. 
Table S1 Kinetic parameters of pyridoxal 5-phosphate adsorption on $\mathrm{Ti}^{4+}$-MAR by pseudo-first-order and pseudo-second-order models.

\begin{tabular}{|c|c|c|c|c|c|c|c|}
\hline \multirow[b]{2}{*}{$\begin{array}{c}\mathrm{C}_{0} \\
(\mathrm{mg} / \mathrm{mL})\end{array}$} & \multirow[b]{2}{*}{$\begin{array}{c}\text { Experimental } \\
Q_{\mathrm{e}}(\mathrm{mg} / \mathrm{g})\end{array}$} & \multicolumn{3}{|c|}{ Pseudo-first-order } & \multicolumn{3}{|c|}{ Pseudo-second-order } \\
\hline & & $\begin{array}{c}k_{1} \\
(1 / \mathrm{min})\end{array}$ & $\begin{array}{c}Q_{1 \text { cal }} \\
(\mathrm{mg} / \mathrm{g})\end{array}$ & $\mathrm{r}_{1}$ & $\begin{array}{c}k_{2} \\
(\mathrm{~g} / \mathrm{mg} \cdot \mathrm{min}) \\
\end{array}$ & $\begin{array}{c}Q_{2 \mathrm{cal}} \\
(\mathrm{mg} / \mathrm{g})\end{array}$ & $\mathrm{r}_{2}$ \\
\hline 0.50 & 33.97 & 0.0636 & 17.63 & 0.9366 & 0.0178 & 32.47 & 0.9988 \\
\hline
\end{tabular}


Table S2 Adsorption isotherm parameters by Freundlich and Langmuir models of pyridoxal 5-phosphate on $\mathrm{Ti}^{4+}$-MAR.

\begin{tabular}{cccccccc}
\hline \multicolumn{3}{c}{ Freundlich isotherm model } & & \multicolumn{3}{c}{ Langmuir isotherm model } \\
\cline { 1 - 2 } \cline { 6 - 8 }$K_{\mathrm{F}}(\mathrm{mg} / \mathrm{g})$ & $1 / n$ & $\mathrm{R}_{\mathrm{F}}$ & & $\mathrm{Q}_{\mathrm{m}}(\mathrm{mg} / \mathrm{g})$ & $K_{\mathrm{L}}(\mathrm{mL} / \mathrm{g})$ & $\mathrm{R}_{\mathrm{L}}$ \\
\hline 46.12 & 0.4722 & 0.9779 & & 60.24 & 3.6889 & 0.9991 \\
\hline
\end{tabular}


Table S3 Enriched phosphopeptides from the tryptic digest of Hela cell proteins with $\mathrm{Ti}^{4+}-\mathrm{MAR}$ as the IMAC sorbent by cLC-MS/MS.

\begin{tabular}{|c|c|c|}
\hline Protein & Peptide sequence & Variable modification \\
\hline Q09666 & VDVKSPK & Phospho(ST) \\
\hline Q09666 & AEAPLPSPK & Phospho(ST) \\
\hline Q09666 & FGFGAKSPK & Phospho(ST) \\
\hline Q09666 & FGTFGGLGSK & Phospho(ST) \\
\hline Q09666 & MPFLSISSPK & Phospho(ST) \\
\hline Q09666 & DIDISSPEFK & Phospho(ST) \\
\hline Q09666 & FKAEAPLPSPK & Phospho(ST) \\
\hline Q09666 & GGVTGSPEASISGSK & Phospho(ST) \\
\hline Q09666 & VKTPEMIIQKPK & Phospho(ST) \\
\hline Q09666 & GHYEVTGSDDETGK & Phospho(ST) \\
\hline Q09666 & ISAPNVDFNLEGPK & Phospho(ST) \\
\hline Q09666 & GKGGVTGSPEASISGSK & Phospho(ST) \\
\hline Q09666 & LPSGSGAASPTGSAVDIR & Phospho(ST) \\
\hline Q09666 & LPSGSGAASPTGSAVDIR & 2 Phospho(ST) \\
\hline Q09666 & ASLGSLEGEAEAEASSPK & Phospho(ST) \\
\hline Q09666 & ASLGSLEGEAEAEASSPK & 2 Phospho(ST) \\
\hline Q09666 & LKSEDGVEGDLGETQSR & Phospho(ST) \\
\hline Q09666 & DDGVFVQEVTQNSPAAR & Phospho(ST) \\
\hline Q09666 & GPSLDIDTPDVNIEGPEGK & Phospho(ST) \\
\hline Q09666 & GKGGVTGSPEASISGSKGDLK & 2 Phospho(ST) \\
\hline Q09666 & SSKASLGSLEGEAEAEASSPK & 2 Phospho(ST) \\
\hline Q09666 & EFSGPSTPTGTLEFEGGEVSLEGGK & 2 Phospho(ST) \\
\hline Q09666 & SKGHYEVTGSDDETGKLQGSGVSLASK & Phospho(ST) \\
\hline Q9H1E3 & ATVTPSPVK & Phospho(ST) \\
\hline Q9H1E3 & ATVTPSPVK & 2 Phospho(ST) \\
\hline Q9H1E3 & ATVTPSPVKGK & Phospho(ST) \\
\hline Q9H1E3 & EEDEEPESPPEK & Phospho(ST) \\
\hline Q9H1E3 & EEDEEPESPPEKK & Phospho(ST) \\
\hline Q9H1E3 & KDDSHSAEDSEDEK & 2 Phospho(ST) \\
\hline Q9H1E3 & NSQEDSEDSEDKDVK & 2 Phospho(ST) \\
\hline Q9H1E3 & TPSPKEEDEEPESPPEKK & Phospho(ST) \\
\hline Q9H1E3 & SGKNSQEDSEDSEDKDVK & 2 Phospho(ST) \\
\hline Q9H1E3 & TPSPKEEDEEPESPPEKK & 2 Phospho(ST) \\
\hline Q9H1E3 & VVDYSQFQESDDADEDYGR & Phospho(ST) \\
\hline Q9H1E3 & KVVDYSQFQESDDADEDYGR & Phospho(ST) \\
\hline Q9H1E3 & VVDYSQFQESDDADEDYGRDSGPPTK & 2 Phospho(ST) \\
\hline P05387 & EESEESDDDMGFGLFD & 2 Phospho(ST) \\
\hline P05387 & KEESEESDDDMGFGLFD & Phospho(ST) \\
\hline P05387 & KEESEESDDDMGFGLFD & 2 Phospho(ST) \\
\hline P05387 & DEKKEESEESDDDMGFGLFD & 2 Phospho(ST) \\
\hline
\end{tabular}




\begin{tabular}{|c|c|c|}
\hline Protein & Peptide sequence & Variable modification \\
\hline P05386 & EESEESDDDMGFGLFD & 2 Phospho(ST) \\
\hline P05386 & KEESEESDDDMGFGLFD & Phospho(ST) \\
\hline P05386 & KEESEESDDDMGFGLFD & 2 Phospho(ST) \\
\hline Q9UQ35 & SRLSLR & Phospho(ST) \\
\hline Q9UQ35 & SRASPVSR & Phospho(ST) \\
\hline Q9UQ35 & SPIRQDR & Phospho(ST) \\
\hline Q9UQ35 & VPSPTPAPK & Phospho(ST) \\
\hline Q9UQ35 & ELSNSPLR & Phospho(ST) \\
\hline Q9UQ35 & SRTSPITR & Phospho(ST) \\
\hline Q9UQ35 & SRSPLAIR & 2 Phospho(ST) \\
\hline Q9UQ35 & SRTPPVTR & 2 Phospho(ST) \\
\hline Q9UQ35 & DGLPRTPSR & Phospho(ST) \\
\hline Q9UQ35 & SLSGSSPCPK & Phospho(ST) \\
\hline Q9UQ35 & GRSPSPKPR & 2 Phospho(ST) \\
\hline Q9UQ35 & HSCSGSSPPR & Phospho(ST) \\
\hline Q9UQ35 & RPSPQPSPR & 2 Phospho(ST) \\
\hline Q9UQ35 & GQSQTSPDHR & Phospho(ST) \\
\hline Q9UQ35 & SSRSSPELTR & Phospho(ST) \\
\hline Q9UQ35 & AQTPPGPSLSGSK & Phospho(ST) \\
\hline Q9UQ35 & YSHSGSSSPDTK & Phospho(ST) \\
\hline Q9UQ35 & SATRPSPSPER & 2 Phospho(ST) \\
\hline Q9UQ35 & SRTPPSAPSQSR & Phospho(ST) \\
\hline Q9UQ35 & ENSFGSPLEFR & Phospho(ST) \\
\hline Q9UQ35 & QSHSGSISPYPK & Phospho(ST) \\
\hline Q9UQ35 & QSHSESPSLQSK & Phospho(ST) \\
\hline Q9UQ35 & SRSRASPATHR & 3 Phospho(ST) \\
\hline Q9UQ35 & SCFESSPDPELK & Phospho(ST) \\
\hline Q9UQ35 & SRSPSSPELNNK & 2 Phospho(ST) \\
\hline Q9UQ35 & RSSRSSPELTR & 3 Phospho(ST) \\
\hline Q9UQ35 & SRSSSPVTELASR & 3 Phospho(ST) \\
\hline Q9UQ35 & ERSGSESSVDQK & 4 Phospho(ST) \\
\hline Q9UQ35 & SSGHSSSELSPDAVEK & Phospho(ST) \\
\hline Q9UQ35 & SRSVSPCSNVESR & 3 Phospho(ST) \\
\hline Q9UQ35 & SSSPVTELASRSPIR & 2 Phospho(ST) \\
\hline Q9UQ35 & SSGHSSSELSPDAVEK & 2 Phospho(ST) \\
\hline Q9UQ35 & THTTALAGRSPSPASGR & 2 Phospho(ST) \\
\hline Q9UQ35 & NHSGSRTPPVALNSSR & 3 Phospho(ST) \\
\hline Q9UQ35 & SSTPPGESYFGVSSLQLK & Phospho(ST) \\
\hline Q9UQ35 & SGSSQELDVKPSASPQER & Phospho(ST) \\
\hline Q9UQ35 & QGSITSPQANEQSVTPQR & Phospho(ST) \\
\hline Q9UQ35 & SGSSQELDVKPSASPQER & 2 Phospho(ST) \\
\hline Q9UQ35 & QGSITSPQANEQSVTPQR & 2 Phospho(ST) \\
\hline Q9UQ35 & SGSSQELDVKPSASPQER & 3 Phospho(ST) \\
\hline
\end{tabular}




\begin{tabular}{|c|c|c|}
\hline Protein & Peptide sequence & Variable modification \\
\hline Q9UQ35 & AQTPPGPSLSGSKSPCPQEK & 2 Phospho(ST) \\
\hline Q9UQ35 & HASSSPESPKPAPAPGSHR & 4 Phospho(ST) \\
\hline Q9UQ35 & QGSITSPQANEQSVTPQRR & 2 Phospho(ST) \\
\hline Q9UQ35 & HASSSPESPKPAPAPGSHR & 5 Phospho(ST) \\
\hline Q9UQ35 & ELSNSPLRENSFGSPLEFR & 2 Phospho(ST) \\
\hline Q9UQ35 & ELSNSPLRENSFGSPLEFR & 3 Phospho(ST) \\
\hline Q9UQ35 & RGEGDAPFSEPGTTSTQRPSSPETATK & Phospho(ST) \\
\hline Q9UQ35 & HGGSPQPLATTPLSQEPVNPPSEASPTR & 2 Phospho(ST) \\
\hline Q9UQ35 & HGGSPQPLATTPLSQEPVNPPSEASPTR & 3 Phospho(ST) \\
\hline Q9UQ35 & HGGSPQPLATTPLSQEPVNPPSEASPTR & 5 Phospho(ST) \\
\hline P06748 & MQASIEK & Phospho(ST) \\
\hline P06748 & DELHIVEAEAMNYEGSPIK & Phospho(ST) \\
\hline P06748 & $\begin{array}{l}\text { CGSGPVHISGQHLVAVEEDAESEDEEEEDV } \\
\mathrm{K}\end{array}$ & Phospho(ST) \\
\hline P08238 & EISDDEAEEEK & Phospho(ST) \\
\hline P08238 & IEDVGSDEEDDSGK & Phospho(ST) \\
\hline P08238 & EKEISDDEAEEEK & Phospho(ST) \\
\hline P08238 & EISDDEAEEEKGEK & Phospho(ST) \\
\hline P08238 & IEDVGSDEEDDSGKDK & Phospho(ST) \\
\hline P08238 & IEDVGSDEEDDSGKDK & 2 Phospho(ST) \\
\hline P08238 & EKEISDDEAEEEKGEK & Phospho(ST) \\
\hline P08238 & IEDVGSDEEDDSGKDKK & Phospho(ST) \\
\hline P07900 & EVSDDEAEEK & Phospho(ST) \\
\hline P07900 & DKEVSDDEAEEK & Phospho(ST) \\
\hline P07900 & ERDKEVSDDEAEEK & Phospho(ST) \\
\hline P07900 & ESEDKPEIEDVGSDEEEEK & Phospho(ST) \\
\hline P07900 & ESEDKPEIEDVGSDEEEEKK & Phospho(ST) \\
\hline P07900 & ESEDKPEIEDVGSDEEEEKKDGDK & Phospho(ST) \\
\hline Q58FF8 & IEDVGSDEEDDSGK & Phospho(ST) \\
\hline Q58FF8 & IEDVGSDEEDDSGKDK & Phospho(ST) \\
\hline Q58FF8 & IEDVGSDEEDDSGKDK & 2 Phospho(ST) \\
\hline Q58FF8 & IEDVGSDEEDDSGKDKK & Phospho(ST) \\
\hline Q58FF7 & EISDDEAEEEK & Phospho(ST) \\
\hline Q58FF7 & EISDDEAEEEKGEK & Phospho(ST) \\
\hline Q8IYB3 & VSHSPPPK & Phospho(ST) \\
\hline Q8IYB3 & TASPPPPPK & Phospho(ST) \\
\hline Q8IYB3 & RRSPSPR & 2 Phospho(ST) \\
\hline Q8IYB3 & AASPSPQSVR & Phospho(ST) \\
\hline Q8IYB3 & HSPSPRPR & 2 Phospho(ST) \\
\hline Q8IYB3 & RYSPPIQR & Phospho(ST) \\
\hline Q8IYB3 & SRVSVSPGR & 2 Phospho(ST) \\
\hline Q8IYB3 & RSPSPPPTR & 2 Phospho(ST) \\
\hline Q8IYB3 & RRSPSLSSK & 2 Phospho(ST) \\
\hline
\end{tabular}




\begin{tabular}{|c|c|c|}
\hline Protein & Peptide sequence & Variable modification \\
\hline Q8IYB3 & KAASPSPQSVR & Phospho(ST) \\
\hline Q8IYB3 & RRTPSPPPR & 2 Phospho(ST) \\
\hline Q8IYB3 & RVSRTPEPK & 2 Phospho(ST) \\
\hline Q8IYB3 & HSPTPQQSNR & Phospho(ST) \\
\hline Q8IYB3 & RHSPSPRPR & 2 Phospho(ST) \\
\hline Q8IYB3 & RASPSPPPKR & 2 Phospho(ST) \\
\hline Q8IYB3 & KAASPSPQSVR & 2 Phospho(ST) \\
\hline Q8IYB3 & RESPSPAPKPR & Phospho(ST) \\
\hline Q8IYB3 & APQTSSSPPPVR & Phospho(ST) \\
\hline Q8IYB3 & RLSPSASPPR & 3 Phospho(ST) \\
\hline Q8IYB3 & RRSPSPPPTR & 2 Phospho(ST) \\
\hline Q8IYB3 & RRSPSPAPPPR & 2 Phospho(ST) \\
\hline Q8IYB3 & APQTSSSPPPVR & 2 Phospho(ST) \\
\hline Q8IYB3 & HRPSPPATPPPK & 2 Phospho(ST) \\
\hline Q8IYB3 & KVELSESEEDKGGK & Phospho(ST) \\
\hline Q8IYB3 & RHSPSRSASPSPR & 4 Phospho(ST) \\
\hline Q8IYB3 & KETESEAEDNLDDLEK & Phospho(ST) \\
\hline Q8IYB3 & KETESEAEDNLDDLEK & 2 Phospho(ST) \\
\hline Q8IYB3 & RVSSSRSVSGSPEPAAK & 5 Phospho(ST) \\
\hline Q8IYB3 & KPPAPPSPVQSQSPSTNWSPAVPVK & Phospho(ST) \\
\hline Q8IYB3 & KPPAPPSPVQSQSPSTNWSPAVPVK & 2 Phospho(ST) \\
\hline Q8IYB3 & KPPAPPSPVQSQSPSTNWSPAVPVK & 3 Phospho(ST) \\
\hline Q8IYB3 & KPPAPPSPVQSQSPSTNWSPAVPVKK & 2 Phospho(ST) \\
\hline Q8IYB3 & KPPAPPSPVQSQSPSTNWSPAVPVKK & 3 Phospho(ST) \\
\hline P02545 & LSPSPTSQR & Phospho(ST) \\
\hline P02545 & LRLSPSPTSQR & Phospho(ST) \\
\hline P02545 & LRLSPSPTSQR & 2 Phospho(ST) \\
\hline P02545 & SGAQASSTPLSPTR & Phospho(ST) \\
\hline P02545 & ASSHSSQTQGGGSVTK & Phospho(ST) \\
\hline P02545 & SVGGSGGGSFGDNLVTR & Phospho(ST) \\
\hline $\mathrm{P} 02545$ & ASSHSSQTQGGGSVTK & 2 Phospho(ST) \\
\hline P02545 & ASASGSGAQVGGPISSGSSASSVTVTR & 2 Phospho(ST) \\
\hline Q9NYF8 & IDISPSTLR & Phospho(ST) \\
\hline Q9NYF8 & RIDISPSTLR & Phospho(ST) \\
\hline Q9NYF8 & AEGEPQEESPLK & Phospho(ST) \\
\hline Q9NYF8 & DLFDYSPPLHK & Phospho(ST) \\
\hline Q9NYF8 & KAEGEPQEESPLK & Phospho(ST) \\
\hline Q9NYF8 & LKDLFDYSPPLHK & Phospho(ST) \\
\hline Q9NYF8 & YSPSQNSPIHHIPSR & Phospho(ST) \\
\hline Q9NYF8 & FNDSEGDDTEETEDYR & Phospho(ST) \\
\hline Q9NYF8 & AEGEWEDQEALDYFSDK & Phospho(ST) \\
\hline Q9NYF8 & SSATSGDIWPGLSAYDNSPR & Phospho(ST) \\
\hline Q9NYF8 & QKFNDSEGDDTEETEDYR & 2 Phospho(ST) \\
\hline
\end{tabular}




\begin{tabular}{|c|c|c|}
\hline Protein & Peptide sequence & Variable modification \\
\hline Q9NYF8 & AEGEWEDQEALDYFSDKESGK & Phospho(ST) \\
\hline Q9NYF8 & GRAEGEWEDQEALDYFSDKESGK & Phospho(ST) \\
\hline P46821 & SDISPLTPR & Phospho(ST) \\
\hline P46821 & КЕTPPKEVK & Phospho(ST) \\
\hline P46821 & VQSLEGEKLSPK & Phospho(ST) \\
\hline P46821 & AAEAGGAEEQYGFLTTPTK & Phospho(ST) \\
\hline P46821 & GEAEQSEEEADEEDKAEDAR & Phospho(ST) \\
\hline P46821 & FEDEGAGFEESSETGDYEEK & Phospho(ST) \\
\hline P46821 & VLSPLRSPPLIGSESAYESFLSADDK & 2 Phospho(ST) \\
\hline Q8NHW5 & EESEESDEDMGFGLFD & Phospho(ST) \\
\hline Q8NHW5 & EESEESDEDMGFGLFD & 2 Phospho(ST) \\
\hline Q8NHW5 & VEAKEESEESDEDMGFGLFD & 2 Phospho(ST) \\
\hline P21333 & CSGPGLSPGMVR & Phospho(ST) \\
\hline P21333 & AFGPGLQGGSAGSPAR & Phospho(ST) \\
\hline P21333 & APSVANVGSHCDLSLK & Phospho(ST) \\
\hline P21333 & RAPSVANVGSHCDLSLK & Phospho(ST) \\
\hline Q13428 & AASAPAKESPR & Phospho(ST) \\
\hline Q13428 & AALAPAKESPR & Phospho(ST) \\
\hline Q13428 & ASSVSTKESPAR & Phospho(ST) \\
\hline Q13428 & TVANLLSGKSPR & Phospho(ST) \\
\hline Q13428 & LDSSPSVSSTLAAK & Phospho(ST) \\
\hline Q13428 & LGAGEGGEASVSPEK & Phospho(ST) \\
\hline Q13428 & TSQVGAASAPAKESPR & Phospho(ST) \\
\hline Q13428 & SLGNILQAKPTSSPAK & Phospho(ST) \\
\hline Q13428 & LGAGEGGEASVSPEKTSTTSK & Phospho(ST) \\
\hline Q13428 & VSDPISTSESSEEEEEAEAETAK & 5 Phospho(ST) \\
\hline Q13428 & $\begin{array}{l}\text { ASESTARSSSSESEDEDVIPATQCLTPGIRTN } \\
\text { VVTMPTAHPR }\end{array}$ & 5 Phospho(ST) \\
\hline P68363 & SIQFVDWCPTGFK & Phospho(ST) \\
\hline P68363 & TIGGGDDSFNTFFSETGAGK & Phospho(ST) \\
\hline P68366 & SIQFVDWCPTGFK & Phospho(ST) \\
\hline Q71U36 & TIGGGDDSFNTFFSETGAGK & Phospho(ST) \\
\hline Q9BQE3 & TIGGGDDSFNTFFSETGAGK & Phospho(ST) \\
\hline Q13748 & TIGGGDDSFNTFFSETGAGK & Phospho(ST) \\
\hline P29401 & ILATPPQEDAPSVDIANIR & Phospho(ST) \\
\hline Q15185 & DWEDDSDEDMSNFDR & Phospho(ST) \\
\hline P05787 & VGSSNFR & Phospho(ST) \\
\hline P05787 & ISSSSFSR & Phospho(ST) \\
\hline P05787 & SYTSGPGSR & Phospho(ST) \\
\hline P05787 & YEELQSLAGK & Phospho(ST) \\
\hline P05787 & SLDMDSIIAEVK & Phospho(ST) \\
\hline P05787 & SLDMDSIIAEVK & 2 Phospho(ST) \\
\hline Q12888 & SEDPPTTPIR & Phospho(ST) \\
\hline
\end{tabular}




\begin{tabular}{|c|c|c|}
\hline Protein & Peptide sequence & Variable modification \\
\hline Q12888 & NSPEDLGLSLTGDSCK & Phospho(ST) \\
\hline Q12888 & STPFIVPSSPTEQEGR & Phospho(ST) \\
\hline Q12888 & SEALSSVLDQEEAMEIK & 3 Phospho(ST) \\
\hline Q12888 & ETAVPGPLGIEDISPNLSPDDK & Phospho(ST) \\
\hline Q12888 & ETAVPGPLGIEDISPNLSPDDK & 2 Phospho(ST) \\
\hline P35579 & GAGDGSDEEVDGK & Phospho(ST) \\
\hline P35579 & KGAGDGSDEEVDGK & Phospho(ST) \\
\hline P35579 & GAGDGSDEEVDGKADGAEAKPAE & Phospho(ST) \\
\hline P35579 & KGAGDGSDEEVDGKADGAEAKPAE & Phospho(ST) \\
\hline Q8WWI1 & VSASLPR & Phospho(ST) \\
\hline Q8WWI1 & GESLDNLDSPR & Phospho(ST) \\
\hline Q8WWI1 & RGESLDNLDSPR & Phospho(ST) \\
\hline Q8WWI1 & SHSPSASQSGSQLR & Phospho(ST) \\
\hline Q8WWI1 & SRSTTELDDYSTNK & Phospho(ST) \\
\hline Q8WWI1 & EGFESDTDSEFTFK & 2 Phospho(ST) \\
\hline Q8WWI1 & LPSPTSPFSSLSQDQAATSK & Phospho(ST) \\
\hline Q8WWI1 & LPSPTSPFSSLSQDQAATSK & 2 Phospho(ST) \\
\hline Q8WWI1 & EVAATEEDVTRLPSPTSPFSSLSQDQAATSK & 2 Phospho(ST) \\
\hline P24534 & DDDDIDLFGSDDEEESEEAK & Phospho(ST) \\
\hline P24534 & DDDDIDLFGSDDEEESEEAKR & Phospho(ST) \\
\hline P24534 & $\begin{array}{l}\text { YGPADVEDTTGSGATDSKDDDDIDLFGSDD } \\
\text { EEESEEAK }\end{array}$ & Phospho(ST) \\
\hline P24534 & $\begin{array}{l}\text { YGPADVEDTTGSGATDSKDDDDIDLFGSDD } \\
\text { EEESEEAKR }\end{array}$ & Phospho(ST) \\
\hline P24534 & $\begin{array}{l}\text { YGPADVEDTTGSGATDSKDDDDIDLFGSDD } \\
\text { EEESEEAKR }\end{array}$ & 2 Phospho(ST) \\
\hline P42167 & AKTPVTLK & Phospho(ST) \\
\hline P42167 & LREQGTESR & Phospho(ST) \\
\hline P42167 & SSTPLPTISSSAENTR & Phospho(ST) \\
\hline P42167 & SSTPLPTISSSAENTR & 2 Phospho(ST) \\
\hline P42167 & HASPILPITEFSDIPR & Phospho(ST) \\
\hline P42167 & GPPDFSSDEEREPTPVLGSGAAAAGR & Phospho(ST) \\
\hline P42167 & GPPDFSSDEEREPTPVLGSGAAAAGR & 2 Phospho(ST) \\
\hline P42167 & GPPDFSSDEEREPTPVLGSGAAAAGR & 3 Phospho(ST) \\
\hline P42166 & LREQGTESR & Phospho(ST) \\
\hline P42166 & SSTPLPTISSSAENTR & Phospho(ST) \\
\hline P42166 & SSTPLPTISSSAENTR & 2 Phospho(ST) \\
\hline P42166 & GPPDFSSDEEREPTPVLGSGAAAAGR & Phospho(ST) \\
\hline P42166 & GPPDFSSDEEREPTPVLGSGAAAAGR & 2 Phospho(ST) \\
\hline P42166 & GPPDFSSDEEREPTPVLGSGAAAAGR & 3 Phospho(ST) \\
\hline P18583 & SFSISPVR & 2 Phospho(ST) \\
\hline P18583 & RRSFSISPVR & 3 Phospho(ST) \\
\hline $\mathrm{P} 18583$ & ESDQTLAALLSPK & Phospho(ST) \\
\hline
\end{tabular}




\begin{tabular}{|c|c|c|}
\hline Protein & Peptide sequence & Variable modification \\
\hline $\mathrm{P} 18583$ & ASESSSEEKDDYEIFVK & 2 Phospho(ST) \\
\hline Q7Z417 & TSPQVLGSILK & Phospho(ST) \\
\hline Q7Z417 & RTSPQVLGSILK & Phospho(ST) \\
\hline Q7Z417 & GADNDGSGSESGYTTPK & Phospho(ST) \\
\hline Q7Z417 & DYEIESQNPLASPTNTLLGSAK & Phospho(ST) \\
\hline P11388 & SVVSDLEADDVK & Phospho(ST) \\
\hline P11388 & SVVSDLEADDVK & 2 Phospho(ST) \\
\hline P11388 & VPDEEENEESDNEK & Phospho(ST) \\
\hline P11388 & NENTEGSPQEDGVELEGLK & Phospho(ST) \\
\hline P11388 & IKNENTEGSPQEDGVELEGLK & Phospho(ST) \\
\hline P11388 & GSVPLSSSPPATHFPDETEITNPVPK & Phospho(ST) \\
\hline P11388 & $\begin{array}{l}\text { FTMDLDSDEDFSDFDEKTDDEDFVPSDASP } \\
\text { PK }\end{array}$ & 3 Phospho(ST) \\
\hline Q9H6F5 & EPGSPPSVQR & Phospho(ST) \\
\hline Q9H6F5 & AGLGSPERPPK & Phospho(ST) \\
\hline Q9H6F5 & QPEYSPESPR & Phospho(ST) \\
\hline Q9H6F5 & LGGLRPESPESLTSVSR & Phospho(ST) \\
\hline Q9H6F5 & AGLGSPERPPKTSPGSPR & 2 Phospho(ST) \\
\hline Q9H6F5 & AGLGSPERPPKTSPGSPR & 3 Phospho(ST) \\
\hline Q9H6F5 & LQQGAGLESPQGQPEPGAASPQR & Phospho(ST) \\
\hline Q9H6F5 & LQQGAGLESPQGQPEPGAASPQR & 2 Phospho(ST) \\
\hline Q8NE71 & LSVPTSDEEDEVPAPKPR & Phospho(ST) \\
\hline Q8NE71 & KLSVPTSDEEDEVPAPKPR & Phospho(ST) \\
\hline Q8NE71 & KLSVPTSDEEDEVPAPKPR & 2 Phospho(ST) \\
\hline Q8NE71 & QQPPEPEWIGDGESTSPSDK & Phospho(ST) \\
\hline Q8NE71 & AEQGSEEEGEGEEEEEEGGESK & Phospho(ST) \\
\hline Q8NE71 & KAEQGSEEEGEGEEEEEEGGESK & Phospho(ST) \\
\hline Q8NE71 & $\begin{array}{l}\text { GGNVFAALIQDQSEEEEEEEKHPPKPAKPE } \\
\mathrm{K}\end{array}$ & Phospho(ST) \\
\hline P29692 & KPATPAEDDEDDDIDLFGSDNEEEDK & Phospho(ST) \\
\hline P29692 & KPATPAEDDEDDDIDLFGSDNEEEDK & 2 Phospho(ST) \\
\hline P29692 & $\begin{array}{l}\text { KPATPAEDDEDDDIDLFGSDNEEEDKEAAQ } \\
\text { LR }\end{array}$ & Phospho(ST) \\
\hline P29692 & $\begin{array}{l}\text { KPATPAEDDEDDDIDLFGSDNEEEDKEAAQ } \\
\text { LR }\end{array}$ & 2 Phospho(ST) \\
\hline P29692 & $\begin{array}{l}\text { KPATPAEDDEDDDIDLFGSDNEEEDKEAAQ } \\
\text { LREER }\end{array}$ & Phospho(ST) \\
\hline P29692 & $\begin{array}{l}\text { KPATPAEDDEDDDIDLFGSDNEEEDKEAAQ } \\
\text { LREER }\end{array}$ & 2 Phospho(ST) \\
\hline Q15149 & RASFAEK & Phospho(ST) \\
\hline Q15149 & AQLEPVASPAK & Phospho(ST) \\
\hline Q15149 & SDEGQLSPATR & Phospho(ST) \\
\hline Q15149 & TPHVQAVQGPLGSPPK & Phospho(ST) \\
\hline
\end{tabular}




\begin{tabular}{|c|c|c|}
\hline Protein & Peptide sequence & Variable modification \\
\hline Q15149 & SSSVGSSSSYPISPAVSR & Phospho(ST) \\
\hline Q15149 & RTPHVQAVQGPLGSPPK & Phospho(ST) \\
\hline Q15149 & SRSSSVGSSSSYPISPAVSR & 3 Phospho(ST) \\
\hline Q15149 & SRSSSVGSSSSYPISPAVSR & 4 Phospho(ST) \\
\hline P49736 & TDALTSSPGR & Phospho(ST) \\
\hline P49736 & TDALTSSPGR & 2 Phospho(ST) \\
\hline P49736 & GNDPLTSSPGR & Phospho(ST) \\
\hline P49736 & RTDALTSSPGR & Phospho(ST) \\
\hline P49736 & GNDPLTSSPGR & 2 Phospho(ST) \\
\hline P49736 & RTDALTSSPGR & 2 Phospho(ST) \\
\hline P49736 & RTDALTSSPGR & 3 Phospho(ST) \\
\hline P49736 & GLLYDSDEEDEERPAR & Phospho(ST) \\
\hline P49736 & RGLLYDSDEEDEERPAR & Phospho(ST) \\
\hline Q96ST2 & ISDSESEDPPR & 2 Phospho(ST) \\
\hline Q96ST2 & TIASDSEEEAGK & 2 Phospho(ST) \\
\hline Q96ST2 & AAVLSDSEDEEK & 2 Phospho(ST) \\
\hline Q96ST2 & GPASDSETEDASR & 2 Phospho(ST) \\
\hline Q96ST2 & KAAVLSDSEDEEK & 2 Phospho(ST) \\
\hline Q96ST2 & AAVLSDSEDEEKASAK & 2 Phospho(ST) \\
\hline Q96ST2 & VVSDADDSDSDAVSDK & 3 Phospho(ST) \\
\hline Q96ST2 & NQASDSENEELPKPR & 2 Phospho(ST) \\
\hline Q96ST2 & HQASDSENEEPPKPR & 2 Phospho(ST) \\
\hline Q96ST2 & HQASDSENEELPKPR & 2 Phospho(ST) \\
\hline Q96ST2 & KAAVLSDSEDEEKASAK & 2 Phospho(ST) \\
\hline P08670 & DGQVINETSQHHDDLE & Phospho(ST) \\
\hline P80723 & AEGAATEEEGTPK & Phospho(ST) \\
\hline P80723 & ETPAATEAPSSTPK & Phospho(ST) \\
\hline P80723 & KAEGAATEEEGTPK & Phospho(ST) \\
\hline Q96JM3 & GGSPDLWK & Phospho(ST) \\
\hline Q96JM3 & KPGPSGPSESPK & Phospho(ST) \\
\hline Q96JM3 & TAPTLSPEHWK & Phospho(ST) \\
\hline Q96JM3 & KTSPASLDFPESQK & Phospho(ST) \\
\hline Q96JM3 & GQESSSDQEQVDVESIDFSK & 2 Phospho(ST) \\
\hline Q96JM3 & GQESSSDQEQVDVESIDFSK & 3 Phospho(ST) \\
\hline Q96JM3 & KPSPSESPEPWKPFPAVSPEPR & Phospho(ST) \\
\hline Q96JM3 & KPSPSESPEPWKPFPAVSPEPR & 2 Phospho(ST) \\
\hline Q02952 & SPPSPVER & Phospho(ST) \\
\hline Q02952 & EGVTPWASFK & 2 Phospho(ST) \\
\hline Q02952 & RPSESDKEDELDK & Phospho(ST) \\
\hline Q02952 & RPSESDKEDELDKVK & Phospho(ST) \\
\hline Q02952 & SAESPTSPVTSETGSTFK & Phospho(ST) \\
\hline Q02952 & RPSESDKEDELDKVK & 2 Phospho(ST) \\
\hline Q02952 & GLAEVQQDGEAEEGATSDGEK & Phospho(ST) \\
\hline
\end{tabular}




\begin{tabular}{|c|c|c|}
\hline Protein & Peptide sequence & Variable modification \\
\hline Q02952 & EVSSLEGSPPPCLGQEEAVCTK & Phospho(ST) \\
\hline O60841 & KWDGSEEDEDNSK & Phospho(ST) \\
\hline O60841 & QSFDDNDSEELEDK & Phospho(ST) \\
\hline O60841 & QSFDDNDSEELEDKDSK & Phospho(ST) \\
\hline O60841 & KQSFDDNDSEELEDKDSK & Phospho(ST) \\
\hline O60841 & VEMYSGSDDDDDFNKLPK & 2 Phospho(ST) \\
\hline O60841 & INSSGESGDESDEFLQSR & 4 Phospho(ST) \\
\hline Q9C0C2 & VNLFPGLSPSALK & Phospho(ST) \\
\hline Q9C0C2 & SQEADVQDWEFR & Phospho(ST) \\
\hline Q9C0C2 & VPSSDEEVVEEPQSR & Phospho(ST) \\
\hline Q9C0C2 & WLDDLLASPPPSGGGAR & Phospho(ST) \\
\hline Q9C0C2 & VPSSDEEVVEEPQSR & 2 Phospho(ST) \\
\hline Q9C0C2 & NRSAEEGELAESKSSQK & Phospho(ST) \\
\hline Q9C0C2 & ASRVPSSDEEVVEEPQSR & 2 Phospho(ST) \\
\hline Q9C0C2 & RDSLGAYASQDANEQGQDLGK & Phospho(ST) \\
\hline Q9C0C2 & TEAQDLCRASPEPPGPESSSR & Phospho(ST) \\
\hline Q9C0C2 & LDSPPPSPITEASEAAEAAEAGNLAVSSR & Phospho(ST) \\
\hline P55081 & IVEPEVVGESDSEVEGDAWR & 2 Phospho(ST) \\
\hline P55081 & EDSSEEEEEEIDDEEIER & 2 Phospho(ST) \\
\hline P55081 & KIVEPEVVGESDSEVEGDAWR & 2 Phospho(ST) \\
\hline P55081 & MEREDSSEEEEEEIDDEEIER & 2 Phospho(ST) \\
\hline P07355 & GLGTDEDSLIEIICSR & Phospho(ST) \\
\hline A6NMY6 & GLGTDEDSLIEIICSR & Phospho(ST) \\
\hline O14974 & TGSYGALAEITASK & Phospho(ST) \\
\hline O14974 & KTGSYGALAEITASK & Phospho(ST) \\
\hline O14974 & RSTQGVTLTDLQEAEK & Phospho(ST) \\
\hline O14974 & STGVSFWTQDSDENEQEQQSDTEEGSNKK & Phospho(ST) \\
\hline Q9BZL4 & TGSSGALGPPER & Phospho(ST) \\
\hline Q9BZL4 & RSTQGVTLTDLKEAEK & Phospho(ST) \\
\hline P07910 & MESEGGADDSAEEGDLLDDDDNEDR & Phospho(ST) \\
\hline P07910 & MESEGGADDSAEEGDLLDDDDNEDR & 2 Phospho(ST) \\
\hline P07910 & $\begin{array}{l}\text { MESEGGADDSAEEGDLLDDDDNEDRGDD } \\
\text { QLELIK }\end{array}$ & Phospho(ST) \\
\hline P07910 & $\begin{array}{l}\text { MESEGGADDSAEEGDLLDDDDNEDRGDD } \\
\text { QLELIKDDEK }\end{array}$ & Phospho(ST) \\
\hline P19338 & VVVSPTK & Phospho(ST) \\
\hline P19338 & VAVATPAK & Phospho(ST) \\
\hline P19338 & AVTTPGKK & Phospho(ST) \\
\hline P19338 & VVVSPTKK & Phospho(ST) \\
\hline P19338 & ALVATPGKK & Phospho(ST) \\
\hline P19338 & KVVVSPTKK & Phospho(ST) \\
\hline P19338 & GFGFVDFNSEEDAK & Phospho(ST) \\
\hline P19338 & EVEEDSEDEEMSEDEEDDSSGEEVVIPQK & 4 Phospho(ST) \\
\hline
\end{tabular}




\begin{tabular}{|c|c|c|}
\hline Protein & Peptide sequence & Variable modification \\
\hline O14950 & ATSNVFAMFDQSQIQEFK & Phospho(ST) \\
\hline P67809 & NEGSESAPEGQAQQR & Phospho(ST) \\
\hline P67809 & NYQQNYQNSESGEKNEGSESAPEGQAQQR & Phospho(ST) \\
\hline P67809 & NYQQNYQNSESGEKNEGSESAPEGQAQQR & 2 Phospho(ST) \\
\hline Q13442 & SLDSDESEDEEDDYQQK & 2 Phospho(ST) \\
\hline Q13442 & SLDSDESEDEEDDYQQK & 3 Phospho(ST) \\
\hline Q13442 & KSLDSDESEDEEDDYQQK & 2 Phospho(ST) \\
\hline Q13442 & SLDSDESEDEEDDYQQKR & 2 Phospho(ST) \\
\hline Q13442 & KSLDSDESEDEEDDYQQKR & 2 Phospho(ST) \\
\hline P18858 & EWNGVVSESDSPVK & Phospho(ST) \\
\hline P18858 & EGEDGDQPTTPPKPLK & Phospho(ST) \\
\hline P18858 & VLGSEGEEEDEALSPAK & Phospho(ST) \\
\hline P18858 & TIQEVLEEQSEDEDR & Phospho(ST) \\
\hline P18858 & VLGSEGEEEDEALSPAK & 2 Phospho(ST) \\
\hline P18858 & QSQIQNQQGEDSGSDPEDTY & 2 Phospho(ST) \\
\hline P18858 & KQSQIQNQQGEDSGSDPEDTY & 2 Phospho(ST) \\
\hline Q9Y2W1 & RRSPSPR & 2 Phospho(ST) \\
\hline Q9Y2W1 & ASAVSELSPR & Phospho(ST) \\
\hline Q9Y2W1 & IDISPSTFR & Phospho(ST) \\
\hline Q9Y2W1 & GSFSDTGLGDGK & Phospho(ST) \\
\hline Q9Y2W1 & RIDISPSTFR & Phospho(ST) \\
\hline Q9Y2W1 & ERSPALKSPLQSVVVR & 2 Phospho(ST) \\
\hline Q9Y2W1 & FSGEEGEIEDDESGTENR & Phospho(ST) \\
\hline Q9Y2W1 & FSGEEGEIEDDESGTENREEK & Phospho(ST) \\
\hline Q9Y2W1 & WAHDKFSGEEGEIEDDESGTENR & Phospho(ST) \\
\hline Q9Y2W1 & WAHDKFSGEEGEIEDDESGTENR & 2 Phospho(ST) \\
\hline Q02880 & KASGSENEGDYNPGR & Phospho(ST) \\
\hline Q02880 & KASGSENEGDYNPGR & 2 Phospho(ST) \\
\hline Q02880 & KVVEAVNSDSDSEFGIPK & 2 Phospho(ST) \\
\hline Q02880 & KTSFDQDSDVDIFPSDFPTEPPSLPR & Phospho(ST) \\
\hline Q02880 & NPWSDDESKSESDLEETEPVVIPR & 4 Phospho(ST) \\
\hline Q02880 & ASPITNDGEDEFVPSDGLDKDEYTFSPGK & Phospho(ST) \\
\hline Q02880 & YTFDFSEEEDDDADDDDDDNNDLEELK & Phospho(ST) \\
\hline Q02880 & $\begin{array}{l}\text { VKASPITNDGEDEFVPSDGLDKDEYTFSPG } \\
\mathrm{K}\end{array}$ & 2 Phospho(ST) \\
\hline P27816 & ASPSKPASAPASR & Phospho(ST) \\
\hline P27816 & RASPSKPASAPASR & Phospho(ST) \\
\hline P27816 & DVTPPPETEVVLIK & Phospho(ST) \\
\hline P27816 & VGSLDNVGHLPAGGAVK & Phospho(ST) \\
\hline Q13283 & SSSPAPADIAQTVQEDLR & Phospho(ST) \\
\hline Q13283 & SSSPAPADIAQTVQEDLR & 2 Phospho(ST) \\
\hline Q13283 & YQDEVFGGFVTEPQEESEEEVEEPEER & Phospho(ST) \\
\hline O75475 & QSNASSDVEVEEK & Phospho(ST) \\
\hline
\end{tabular}




\begin{tabular}{|c|c|c|}
\hline Protein & Peptide sequence & Variable modification \\
\hline O75475 & TGVTSTSDSEEEGDDQEGEK & 2 Phospho(ST) \\
\hline O75475 & TGVTSTSDSEEEGDDQEGEK & 3 Phospho(ST) \\
\hline O75475 & TGVTSTSDSEEEGDDQEGEKK & 2 Phospho(ST) \\
\hline O75475 & ETSVSKEDTDHEEKASNEDVTK & Phospho(ST) \\
\hline P18615 & SGAHSSASPPR & Phospho(ST) \\
\hline P18615 & SVWGSLAVQNSPK & Phospho(ST) \\
\hline P18615 & SISADDDLQESSR & Phospho(ST) \\
\hline P27824 & AEEDEILNRSPR & Phospho(ST) \\
\hline P27824 & SDAEEDGGTVSQEEEDR & Phospho(ST) \\
\hline P27824 & QKSDAEEDGGTVSQEEEDR & Phospho(ST) \\
\hline P27824 & QKSDAEEDGGTVSQEEEDR & 2 Phospho(ST) \\
\hline P27824 & SDAEEDGGTVSQEEEDRKPK & Phospho(ST) \\
\hline P27824 & QKSDAEEDGGTVSQEEEDRKPK & Phospho(ST) \\
\hline Q5T200 & FVHGPSPR & Phospho(ST) \\
\hline Q5T200 & KEVSPEVVR & Phospho(ST) \\
\hline Q5T200 & SPERPTGDLR & Phospho(ST) \\
\hline Q5T200 & GNIETTSEDGQVFSPK & Phospho(ST) \\
\hline Q5T200 & TPSPPPPIPEDIALGK & 2 Phospho(ST) \\
\hline Q5T200 & SASPYPSHSLSSPQR & 2 Phospho(ST) \\
\hline Q5T200 & SKGDSDISDEEAAQQSK & 2 Phospho(ST) \\
\hline Q5T200 & KEDTAFSDWSDEDVPDR & 2 Phospho(ST) \\
\hline Q5T200 & $\begin{array}{l}\text { LDDAHSLGSGAGEGYEPISDDELDEILAGD } \\
\text { AEK }\end{array}$ & 3 Phospho(ST) \\
\hline Q6PD62 & KGSGSEQEGEDEEGGER & 2 Phospho(ST) \\
\hline Q6PD62 & AIISSSDDSSDEDKLK & 5 Phospho(ST) \\
\hline Q6PD62 & GGEFDEFVNDDTDDDLPISK & Phospho(ST) \\
\hline Q6PD62 & KGGEFDEFVNDDTDDDLPISK & Phospho(ST) \\
\hline P17096 & KQPPVSPGTALVGSQK & Phospho(ST) \\
\hline P17096 & EEEEGISQESSEEEQ & 2 Phospho(ST) \\
\hline P17096 & EEEEGISQESSEEEQ & 3 Phospho(ST) \\
\hline P17096 & LEKEEEEGISQESSEEEQ & 2 Phospho(ST) \\
\hline P17096 & KLEKEEEEGISQESSEEEQ & Phospho(ST) \\
\hline P17096 & KLEKEEEEGISQESSEEEQ & 2 Phospho(ST) \\
\hline P17096 & KLEKEEEEGISQESSEEEQ & 3 Phospho(ST) \\
\hline P17096 & KQPPVSPGTALVGSQKEPSEVPTPK & Phospho(ST) \\
\hline P17096 & KQPPVSPGTALVGSQKEPSEVPTPK & 2 Phospho(ST) \\
\hline Q9UPQ0 & SDSLSPPR & Phospho(ST) \\
\hline Q9UPQ0 & QTPSPDVVLR & Phospho(ST) \\
\hline Q9UPQ0 & SINHQIESPSER & Phospho(ST) \\
\hline Q9UPQ0 & SRQTPSPDVVLR & 2 Phospho(ST) \\
\hline Q9UPQ0 & SPEPEATLTFPFLDK & Phospho(ST) \\
\hline Q9UPQ0 & GSSDGRGSDSESDLPHR & Phospho(ST) \\
\hline Q9UPQ0 & GSSDGRGSDSESDLPHR & 2 Phospho(ST) \\
\hline
\end{tabular}




\begin{tabular}{|c|c|c|}
\hline Protein & Peptide sequence & Variable modification \\
\hline Q9UPQ0 & CSPTVAFVEFPSSPQLK & Phospho(ST) \\
\hline Q9UPQ0 & VVKPKSPEPEATLTFPFLDK & Phospho(ST) \\
\hline Q9Y6G9 & SVSSNVASVSPIPAGSK & Phospho(ST) \\
\hline Q9Y6G9 & KPVTVSPTTPTSPTEGEAS & Phospho(ST) \\
\hline Q9Y6G9 & DFQEYVEPGEDFPASPQR & Phospho(ST) \\
\hline O00567 & EELMSSDLEETAGSTSIPK & Phospho(ST) \\
\hline P51858 & AGDLLEDSPK & Phospho(ST) \\
\hline P51858 & RAGDLLEDSPK & Phospho(ST) \\
\hline P51858 & AGDLLEDSPKRPK & Phospho(ST) \\
\hline P51858 & RAGDLLEDSPKRPK & Phospho(ST) \\
\hline P51858 & GNAEGSSDEEGKLVIDEPAK & Phospho(ST) \\
\hline P51858 & GNAEGSSDEEGKLVIDEPAK & 2 Phospho(ST) \\
\hline P51858 & KGNAEGSSDEEGKLVIDEPAK & 2 Phospho(ST) \\
\hline P51858 & GNAEGSSDEEGKLVIDEPAKEK & 2 Phospho(ST) \\
\hline P51858 & $\begin{array}{l}\text { SCVEEPEPEPEAAEGDGDKKGNAEGSSDEE } \\
\text { GK }\end{array}$ & 2 Phospho(ST) \\
\hline Q76FK4 & NSISDDDTDSEDELR & 3 Phospho(ST) \\
\hline Q76FK4 & FLETDSEEEQEEVNEK & 2 Phospho(ST) \\
\hline Q76FK4 & LQDSSSEEEDVTEETDHR & 3 Phospho(ST) \\
\hline P47712 & CSVSLSNVEAR & Phospho(ST) \\
\hline P47712 & HIVSNDSSDSDDESHEPK & Phospho(ST) \\
\hline P47712 & HIVSNDSSDSDDESHEPK & 2 Phospho(ST) \\
\hline P47712 & HIVSNDSSDSDDESHEPK & 3 Phospho(ST) \\
\hline Q13895 & MPQDGSDDEDEEWPTLEK & Phospho(ST) \\
\hline O00193 & SASPDDDLGSSNWEAADLGNEER & Phospho(ST) \\
\hline O00193 & SASPDDDLGSSNWEAADLGNEERK & Phospho(ST) \\
\hline O00193 & RSASPDDDLGSSNWEAADLGNEER & Phospho(ST) \\
\hline Q13085 & FIIGSVSEDNSEDEISNLVK & Phospho(ST) \\
\hline Q13085 & FIIGSVSEDNSEDEISNLVK & 2 Phospho(ST) \\
\hline Q16637 & SDNIKPK & Phospho(ST) \\
\hline Q16637 & GTGQSDDSDIWDDTALIK & 2 Phospho(ST) \\
\hline Q16637 & RGTGQSDDSDIWDDTALIK & Phospho(ST) \\
\hline Q16637 & RGTGQSDDSDIWDDTALIK & 2 Phospho(ST) \\
\hline Q16637 & RGTGQSDDSDIWDDTALIK & 3 Phospho(ST) \\
\hline Q9Y6E2 & FVEWLQNAEEESESEGEEN & 2 Phospho(ST) \\
\hline Q9Y6E2 & KFVEWLQNAEEESESEGEEN & 2 Phospho(ST) \\
\hline Q6PKG0 & SLPTTVPESPNYR & Phospho(ST) \\
\hline Q6PKG0 & SLPTTVPESPNYR & 2 Phospho(ST) \\
\hline Q6PKG0 & AVTPVPTKTEEVSNLK & Phospho(ST) \\
\hline Q6PKG0 & NTFTAWSDEESDYEIDDR & Phospho(ST) \\
\hline Q6PKG0 & KNTFTAWSDEESDYEIDDR & 2 Phospho(ST) \\
\hline Q9Y2X3 & EEPLSEEEPCTSTAIASPEK & Phospho(ST) \\
\hline Q9Y2X3 & HIKEEPLSEEEPCTSTAIASPEK & Phospho(ST) \\
\hline
\end{tabular}




\begin{tabular}{|c|c|c|}
\hline Protein & Peptide sequence & Variable modification \\
\hline Q9Y2X3 & HIKEEPLSEEEPCTSTAIASPEK & 2 Phospho(ST) \\
\hline Q9Y2X3 & HIKEEPLSEEEPCTSTAIASPEKK & 2 Phospho(ST) \\
\hline O43719 & DLDEEGSEK & Phospho(ST) \\
\hline O43719 & VLDEEGSER & Phospho(ST) \\
\hline O43719 & EFDEDSDEKEEEEDTYEK & Phospho(ST) \\
\hline O43719 & LFDEEEDSSEKLFDDSDER & 2 Phospho(ST) \\
\hline O43719 & LFEDDDSNEKLFDEEEDSSEK & Phospho(ST) \\
\hline O43719 & LFEESDDKEDEDADGKEVEDADEK & Phospho(ST) \\
\hline O43719 & VLDEEGSEREFDEDSDEKEEEEDTYEK & 2 Phospho(ST) \\
\hline O43719 & LFEDDDSNEKLFDEEEDSSEKLFDDSDER & 3 Phospho(ST) \\
\hline P35221 & SRTSVQTEDDQLIAGQSAR & 2 Phospho(ST) \\
\hline P35221 & TPEELDDSDFETEDFDVR & Phospho(ST) \\
\hline P26232 & SRTSVQTEDDQLIAGQSAR & 2 Phospho(ST) \\
\hline P29966 & LSGFSFK & Phospho(ST) \\
\hline P29966 & AEDGATPSPSNETPK & Phospho(ST) \\
\hline P29966 & GEAAAERPGEAAVASSPSK & Phospho(ST) \\
\hline P29966 & $\begin{array}{l}\text { EAPAEGEAAEPGSPTAAEGEAASAASSTSSP } \\
\mathrm{K}\end{array}$ & Phospho(ST) \\
\hline P29966 & $\begin{array}{l}\text { EAPAEGEAAEPGSPTAAEGEAASAASSTSSP } \\
\mathrm{K}\end{array}$ & 2 Phospho(ST) \\
\hline Q9NTJ3 & TESPATAAETASEELDNR & Phospho(ST) \\
\hline Q9NTJ3 & EEGPPPPSPDGASSDAEPEPPSGR & Phospho(ST) \\
\hline Q9NTJ3 & REEGPPPPSPDGASSDAEPEPPSGR & Phospho(ST) \\
\hline Q9NTJ3 & RREEGPPPPSPDGASSDAEPEPPSGR & 2 Phospho(ST) \\
\hline Q9Y4F1 & LGAPENSGISTLER & Phospho(ST) \\
\hline Q9Y4F1 & VSAGEPGSHPSPAPR & Phospho(ST) \\
\hline Q9Y4F1 & SPDEATAADQESEDDLSASR & Phospho(ST) \\
\hline Q9Y4F1 & SPDEATAADQESEDDLSASRTSLER & Phospho(ST) \\
\hline Q99543 & NASASFQELEDK & Phospho(ST) \\
\hline Q99543 & NASASFQELEDK & 2 Phospho(ST) \\
\hline Q99543 & NASASFQELEDKK & 2 Phospho(ST) \\
\hline Q99543 & KELSEESEDEELQLEEFPMLK & 2 Phospho(ST) \\
\hline Q13435 & SSLGQSASETEEDTVSVSK & 2 Phospho(ST) \\
\hline Q13435 & SSLGQSASETEEDTVSVSK & 3 Phospho(ST) \\
\hline Q13435 & SSLGQSASETEEDTVSVSKK & 2 Phospho(ST) \\
\hline Q13435 & GFEEEHKDSDDDSSDDEQEKKPEAPK & 3 Phospho(ST) \\
\hline P17812 & SGSSSPDSEITELK & Phospho(ST) \\
\hline P17812 & SGSSSPDSEITELK & 2 Phospho(ST) \\
\hline P17812 & SGSSSPDSEITELK & 3 Phospho(ST) \\
\hline P17812 & SGSSSPDSEITELKFPSINHD & Phospho(ST) \\
\hline P17812 & SGSSSPDSEITELKFPSINHD & 2 Phospho(ST) \\
\hline P17812 & DTYSDRSGSSSPDSEITELK & 3 Phospho(ST) \\
\hline $\mathrm{P} 17812$ & DTYSDRSGSSSPDSEITELKFPSINHD & Phospho(ST) \\
\hline
\end{tabular}




\begin{tabular}{|c|c|c|}
\hline Protein & Peptide sequence & Variable modification \\
\hline P54105 & EPVADEEEEDSDDDVEPITEFR & Phospho(ST) \\
\hline P54105 & FEEESKEPVADEEEEDSDDDVEPITEFR & Phospho(ST) \\
\hline O75122 & SRSDIDVNAAAGAK & Phospho(ST) \\
\hline O75122 & IPRPSVSQGCSR & 2 Phospho(ST) \\
\hline O75122 & VLNTGSDVEEAVADALK & Phospho(ST) \\
\hline P61978 & GSYGDLGGPIITTQVTIPK & Phospho(ST) \\
\hline O00264 & EGEEPTVYSDEEEPK & Phospho(ST) \\
\hline O00264 & GDQPAASGDSDDDEPPPLPR & Phospho(ST) \\
\hline O00264 & EGEEPTVYSDEEEPKDESAR & Phospho(ST) \\
\hline O00264 & IVRGDQPAASGDSDDDEPPPLPR & Phospho(ST) \\
\hline Q0ZGT2 & KESLSPGK & Phospho(ST) \\
\hline Q0ZGT2 & EMLASDDEEDVSSK & Phospho(ST) \\
\hline Q99590 & TEELIESPK & Phospho(ST) \\
\hline Q99590 & KRPQSPSPR & 2 Phospho(ST) \\
\hline Q99590 & FHSPSTTWSPNKDTPQEK & 2 Phospho(ST) \\
\hline Q99590 & SSSNDSVDEETAESDTSPVLEK & Phospho(ST) \\
\hline Q99590 & SPISDNSGCDAPGNSNPSLSVPSSAESEK & Phospho(ST) \\
\hline Q99590 & $\begin{array}{l}\text { AETASQSQRSPISDNSGCDAPGNSNPSLSVP } \\
\text { SSAESEK }\end{array}$ & Phospho(ST) \\
\hline Q9Y3T9 & DLFDLNSSEEDDTEGFSER & 2 Phospho(ST) \\
\hline Q9Y3T9 & QFKDLFDLNSSEEDDTEGFSER & 2 Phospho(ST) \\
\hline O43166 & TLSDESIYNSQR & Phospho(ST) \\
\hline O43166 & NSPSNLSSSSDTGSVGGTYR & Phospho(ST) \\
\hline Q8N163 & SVASNQSEMEFSSLQDMPK & 3 Phospho(ST) \\
\hline Q7Z460 & SRSDIDVNAAASAK & Phospho(ST) \\
\hline Q7Z460 & RQSSGSATNVASTPDNR & Phospho(ST) \\
\hline P41236 & IQEQESSGEEDSDLSPEER & Phospho(ST) \\
\hline P41236 & IQEQESSGEEDSDLSPEER & 2 Phospho(ST) \\
\hline P22626 & GGNFGFGDSR & Phospho(ST) \\
\hline P16402 & KVAGAATPK & Phospho(ST) \\
\hline P16402 & KASGPPVSELITK & Phospho(ST) \\
\hline P16403 & KAAGGATPK & Phospho(ST) \\
\hline P16403 & KPAAATVTK & Phospho(ST) \\
\hline P16403 & KASGPPVSELITK & Phospho(ST) \\
\hline P10412 & KASGPPVSELITK & Phospho(ST) \\
\hline Q5JTV8 & SRDSDESGDK & Phospho(ST) \\
\hline Q5JTV8 & SELGNQSPSTSSR & Phospho(ST) \\
\hline Q5JTV8 & LQQQHSEQPPLQPSPVMTR & Phospho(ST) \\
\hline Q5JTV8 & DSHSSEEDEASSQTDLSQTISK & 2 Phospho(ST) \\
\hline Q5JTV8 & VNFSEEGETEEDDQDSSHSSVTTVK & 2 Phospho(ST) \\
\hline Q14247 & LPSSPVYEDAASFK & Phospho(ST) \\
\hline Q14247 & TQTPPVSPAPQPTEER & Phospho(ST) \\
\hline Q14247 & TQTPPVSPAPQPTEER & 2 Phospho(ST) \\
\hline
\end{tabular}




\begin{tabular}{|c|c|c|}
\hline Protein & Peptide sequence & Variable modification \\
\hline Q14247 & AKTQTPPVSPAPQPTEER & Phospho(ST) \\
\hline Q14247 & AKTQTPPVSPAPQPTEER & 2 Phospho(ST) \\
\hline Q8NHQ9 & TVDLGISDLEDDC & Phospho(ST) \\
\hline Q8NHQ9 & EEGSDIEDEDMEELLNDTR & Phospho(ST) \\
\hline Q9UK76 & NSSEASSGDFLDLK & Phospho(ST) \\
\hline Q9UK76 & SSGGREDLESSGLQR & Phospho(ST) \\
\hline Q9UK76 & RNSSEASSGDFLDLK & Phospho(ST) \\
\hline P13861 & GDSESEEDEDLEVPVPSR & 2 Phospho(ST) \\
\hline P13861 & RVSVCAETYNPDEEEEDTDPR & Phospho(ST) \\
\hline P13861 & VADAKGDSESEEDEDLEVPVPSR & 2 Phospho(ST) \\
\hline Q8WVC0 & KLTSDEEGEPSGK & Phospho(ST) \\
\hline Q8WVC0 & KLTSDEEGEPSGK & 2 Phospho(ST) \\
\hline Q8WVC0 & QNSDDEEQPQLSDEEK & 2 Phospho(ST) \\
\hline Q8WVC0 & AQGSDEDKLQNSDDDEK & 2 Phospho(ST) \\
\hline Q8WVC0 & EDKSDQSDDEKIQNSDDEER & 3 Phospho(ST) \\
\hline Q13459 & RTSFSTSDVSK & Phospho(ST) \\
\hline Q13459 & GSDEENLDSETSASTESLLEER & Phospho(ST) \\
\hline Q9BVS4 & AEVYGSENESER & Phospho(ST) \\
\hline Q9BVS4 & EGSEFSFSDGEVAEK & 3 Phospho(ST) \\
\hline Q9H7N4 & REVLYDSEGLSGEER & 2 Phospho(ST) \\
\hline Q9H7N4 & QRSPSPAPAPAPAAAAGPPTR & 2 Phospho(ST) \\
\hline Q9H7N4 & EAASSSSGTQPAPPAPASPWDSK & Phospho(ST) \\
\hline Q9H7N4 & FDIYDPFHPTDEAYSPPPAPEQK & Phospho(ST) \\
\hline Q5JSH3 & VGNESPVQELK & Phospho(ST) \\
\hline Q5JSH3 & LTQTSSTEQLNVLETETEVLNK & Phospho(ST) \\
\hline P20700 & LKLSPSPSSR & Phospho(ST) \\
\hline P20700 & TTIPEEEEEEEEAAGVVVEEELFHQQGTPR & Phospho(ST) \\
\hline Q03252 & LKLSPSPSSR & Phospho(ST) \\
\hline O43815 & FLESAAADFSDEDEDDDVDGR & Phospho(ST) \\
\hline Q15276 & AQSTDSLGTSGSLQSK & Phospho(ST) \\
\hline Q15276 & RAQSTDSLGTSGSLQSK & 2 Phospho(ST) \\
\hline P05783 & LLEDGEDFNLGDALDSSNSMQTIQK & Phospho(ST) \\
\hline P46087 & GPQPPTVSPIR & Phospho(ST) \\
\hline P46087 & GTDTQTPAVLSPSK & Phospho(ST) \\
\hline P46087 & EAAAGIQWSEEETEDEEEEK & 2 Phospho(ST) \\
\hline P46087 & EAAAGIQWSEEETEDEEEEKEVTPESGPPK & 2 Phospho(ST) \\
\hline O15173 & GLGAGAGAGEESPATSLPR & Phospho(ST) \\
\hline O15173 & LLKPGEEPSEYTDEEDTK & Phospho(ST) \\
\hline O15173 & LLKPGEEPSEYTDEEDTKDHNKQD & Phospho(ST) \\
\hline Q7Z4V5 & ARGDSEALDEES & Phospho(ST) \\
\hline Q7Z4V5 & EGPDLDRPGSDR & Phospho(ST) \\
\hline Q7Z4V5 & GEAERGSGGSSGDELR & 3 Phospho(ST) \\
\hline Q7Z4V5 & GRGPPSSSDSEPEAELER & 4 Phospho(ST) \\
\hline
\end{tabular}




\begin{tabular}{|c|c|c|}
\hline Protein & Peptide sequence & Variable modification \\
\hline Q7Z4V5 & GEAERGSGGSSGDELREDDEPVK & 3 Phospho(ST) \\
\hline P49792 & YSLSPSK & Phospho(ST) \\
\hline P49792 & SALSPSKSPAK & Phospho(ST) \\
\hline P49792 & FESPATGILSPR & Phospho(ST) \\
\hline P49792 & LHDSSGSQVGTGFK & Phospho(ST) \\
\hline P49792 & LNQSGTSVGTDEESDVTQEEER & Phospho(ST) \\
\hline P49792 & EDALDDSVSSSSVHASPLASSPVRK & 3 Phospho(ST) \\
\hline P49792 & ELVGPPLAETVFTPKTSPENVQDR & 2 Phospho(ST) \\
\hline P49792 & $\begin{array}{l}\text { NHETDGGSAHGDDDDDGPHFEPVVPLPDKI } \\
\text { EVK }\end{array}$ & Phospho(ST) \\
\hline P49792 & $\begin{array}{l}\text { VGEDEDGSDEEVVHNEDIHFEPIVSLPEVEV } \\
\mathrm{K}\end{array}$ & Phospho(ST) \\
\hline Q7Z3J3 & YSLSPSK & Phospho(ST) \\
\hline Q7Z3J3 & SALSPSKSPAK & Phospho(ST) \\
\hline Q7Z3J3 & LNQSGTSVGTDEESDVTQEEER & Phospho(ST) \\
\hline Q7Z3J3 & EDALDDSVSSSSVHASPLASSPVRK & 3 Phospho(ST) \\
\hline A6NKT7 & YSLSPSK & Phospho(ST) \\
\hline A6NKT7 & LNQSGTSVGTDEESDVTQEEER & Phospho(ST) \\
\hline A6NKT7 & EDALDDSVSSSSVHASPLASSPVRK & 3 Phospho(ST) \\
\hline P0DJD0 & YSLSPSK & Phospho(ST) \\
\hline P0DJD0 & LNQSGTSVGTDEESDVTQEEER & Phospho(ST) \\
\hline O14715 & YSLSPSK & Phospho(ST) \\
\hline O14715 & EDALDDSVSSSSVHASPLASSPVRK & 3 Phospho(ST) \\
\hline Q9NR30 & KKEEPSQNDISPK & Phospho(ST) \\
\hline Q9NR30 & NEEPSEEEIDAPKPK & Phospho(ST) \\
\hline P25205 & SEDESETEDEEEK & Phospho(ST) \\
\hline P25205 & SEDESETEDEEEKSQEDQEQK & 2 Phospho(ST) \\
\hline P25205 & DGDSYDPYDFSDTEEEMPQVHTPK & Phospho(ST) \\
\hline P25205 & DGDSYDPYDFSDTEEEMPQVHTPK & 2 Phospho(ST) \\
\hline O95817 & SSTPLHSPSPIR & 2 Phospho(ST) \\
\hline O95817 & SQSPAASDCSSSSSSASLPSSGR & Phospho(ST) \\
\hline O95817 & VPPAPVPCPPPSPGPSAVPSSPK & 2 Phospho(ST) \\
\hline P46013 & SGASEANLIVAK & Phospho(ST) \\
\hline P46013 & LDLTENLTGSK & Phospho(ST) \\
\hline P46013 & IPCKSSPELEDTATSSK & Phospho(ST) \\
\hline P46013 & ILCKSPQSDPADTPTNTK & 2 Phospho(ST) \\
\hline P46013 & SGGSGHAVAEPASPEQELDQNK & Phospho(ST) \\
\hline Q04726 & ESSANNSVSPSESLR & Phospho(ST) \\
\hline Q04726 & DAPTSPASVASSSSTPSSK & Phospho(ST) \\
\hline Q12802 & SGSLDSELSVSPK & Phospho(ST) \\
\hline Q12802 & FLSHSTDSLNK & 2 Phospho(ST) \\
\hline Q12802 & VDSLVSLSEEDLESDQR & 2 Phospho(ST) \\
\hline Q96CX2 & SPSGGAAGPLLTPSQSLDGSR & Phospho(ST) \\
\hline
\end{tabular}




\begin{tabular}{|c|c|c|}
\hline Protein & Peptide sequence & Variable modification \\
\hline P63104 & TAFDEAIAELDTLSEESYK & Phospho(ST) \\
\hline Q8NFC6 & TDTGIVTVEQSPSSSK & Phospho(ST) \\
\hline Q8NFC6 & YYSDSDDELTVEQR & 2 Phospho(ST) \\
\hline Q8NFC6 & YYSDSDDELTVEQR & Phospho(ST) \\
\hline Q8NFC6 & KEQESDEEEEEEEEDEPSGATTR & Phospho(ST) \\
\hline P26358 & EADDDEEVDDNIPEMPSPK & Phospho(ST) \\
\hline P20810 & SESELIDELSEDFDR & Phospho(ST) \\
\hline Q15019 & IYHLPDAESDEDEDFK & Phospho(ST) \\
\hline Q15019 & IYHLPDAESDEDEDFKEQTR & Phospho(ST) \\
\hline Q15811 & SAFTPATATGSSPSPVLGQGEK & Phospho(ST) \\
\hline Q8WXI9 & GRLTPSPDIIVLSDNEASSPR & 3 Phospho(ST) \\
\hline Q8WXI9 & GRLTPSPDIIVLSDNEASSPR & 4 Phospho(ST) \\
\hline P46100 & ETFSSAEGTVDK & Phospho(ST) \\
\hline P46100 & EVIEIEDASPTK & Phospho(ST) \\
\hline P46100 & KSSTSGSDFDTK & 2 Phospho(ST) \\
\hline P46100 & RPTETNPVTSNSDEECNETVK & Phospho(ST) \\
\hline P46100 & RPTETNPVTSNSDEECNETVK & 3 Phospho(ST) \\
\hline Q16512 & LNLGTDSDSSPQK & Phospho(ST) \\
\hline Q16512 & LNLGTDSDSSPQK & 2 Phospho(ST) \\
\hline Q16512 & TDVSNFDEEFTGEAPTLSPPR & Phospho(ST) \\
\hline O60343 & LGSVDSFER & Phospho(ST) \\
\hline O60343 & SLTSSLENIFSR & Phospho(ST) \\
\hline O60343 & TSSTCSNESLSVGGTSVTPR & 3 Phospho(ST) \\
\hline Q96D46 & DSAIPVESDTDDEGAPR & Phospho(ST) \\
\hline Q96D46 & DSAIPVESDTDDEGAPR & 2 Phospho(ST) \\
\hline P35659 & KESESEDSSDDEPLIK & 4 Phospho(ST) \\
\hline P35659 & KESESEDSSDDEPLIKK & 4 Phospho(ST) \\
\hline P35659 & EESEEEEDEDDEEEEEEEK & Phospho(ST) \\
\hline Q9UN86 & STTPPPAEPVSLPQEPPK & Phospho(ST) \\
\hline Q9UN86 & YEDEVFGDSEPELDEESEDEVEEEQEER & Phospho(ST) \\
\hline Q9UN86 & YEDEVFGDSEPELDEESEDEVEEEQEER & 2 Phospho(ST) \\
\hline O94979 & AQGEPVAGHESPK & Phospho(ST) \\
\hline O94979 & AQGEPVAGHESPKIPYEK & Phospho(ST) \\
\hline O94979 & DSDQVAQSDGEESPAAEEQLLGEHIK & Phospho(ST) \\
\hline O94979 & DSDQVAQSDGEESPAAEEQLLGEHIKEEK & Phospho(ST) \\
\hline P35269 & GNSRPGTPSAEGGSTSSTLR & 2 Phospho(ST) \\
\hline P35269 & LDTGPQSLSGKSTPQPPSGK & 2 Phospho(ST) \\
\hline P35269 & GVDEQSDSSEESEEEKPPEEDKEEEEEK & 4 Phospho(ST) \\
\hline Q15154 & QAESLSLTR & Phospho(ST) \\
\hline Q15154 & WVSELSYVEEK & Phospho(ST) \\
\hline Q15154 & QGLAETASPVAVSLR & 2 Phospho(ST) \\
\hline Q15154 & VTNDISPESSPGVGR & 3 Phospho(ST) \\
\hline Q15154 & KDEETEESEYDSEHENSEPVTNIR & 2 Phospho(ST) \\
\hline
\end{tabular}




\begin{tabular}{|c|c|c|}
\hline Protein & Peptide sequence & Variable modification \\
\hline Q15154 & NVRSDISDQEEDEESEGCPVSINLSK & 3 Phospho(ST) \\
\hline Q9H3N1 & SLGPSLATDK & Phospho(ST) \\
\hline Q9H3N1 & VEEEQEADEEDVSEEEAESK & Phospho(ST) \\
\hline P23193 & EPAITSQNSPEAR & Phospho(ST) \\
\hline P23193 & KEPAITSQNSPEAR & Phospho(ST) \\
\hline P23193 & KKEPAITSQNSPEAR & Phospho(ST) \\
\hline Q8N3X1 & ALEEGDGSVSGSSPR & Phospho(ST) \\
\hline Q8NC51 & FDQLFDDESDPFEVLK & Phospho(ST) \\
\hline P45973 & TADSSSSEDEEEYVVEK & 4 Phospho(ST) \\
\hline P45973 & RTADSSSSEDEEEYVVEK & 4 Phospho(ST) \\
\hline Q6NZI2 & RGSSPDVHALLEITEESDAVLVDKSDSD & 4 Phospho(ST) \\
\hline Q6NZI2 & $\begin{array}{l}\text { EGEELGEGERPEEDAAALELSSDEAVEVEE } \\
\text { VIEESR }\end{array}$ & 2 Phospho(ST) \\
\hline Q6NZI2 & $\begin{array}{l}\text { ESEALPEKEGEELGEGERPEEDAAALELSSD } \\
\text { EAVEVEEVIEESR }\end{array}$ & 2 Phospho(ST) \\
\hline P46379 & ENASPAPGTTAEEAMSR & Phospho(ST) \\
\hline P46379 & $\begin{array}{l}\text { APPQTHLPSGASSGTGSASATHGGGSPPGT } \\
\mathrm{R}\end{array}$ & Phospho(ST) \\
\hline Q96KC8 & DFDIAEQNESSDEESLR & 2 Phospho(ST) \\
\hline Q96KC8 & DFDIAEQNESSDEESLRK & 2 Phospho(ST) \\
\hline Q96KC8 & EDAEGVAAEEEQEGDSGEQETGATDARPR & Phospho(ST) \\
\hline Q15459 & EKQSDDEVYAPGLDIESSLK & Phospho(ST) \\
\hline Q15459 & FGESEEVEMEVESDEEDDKQEK & Phospho(ST) \\
\hline P16401 & AVKKTPK & Phospho(ST) \\
\hline P16401 & SETAPAETATPAPVEKSPAK & Phospho(ST) \\
\hline Q04695 & QFTSSSSIK & Phospho(ST) \\
\hline Q04695 & LSGGLGAGSCR & Phospho(ST) \\
\hline Q04695 & TSCRLSGGLGAGSCR & Phospho(ST) \\
\hline O43823 & VDSEGDFSENDDAAGDFR & Phospho(ST) \\
\hline $\mathrm{O} 43823$ & VDSEGDFSENDDAAGDFR & 2 Phospho(ST) \\
\hline Q96ST3 & GDLSDVEEEEEEEMDVDEATGAVK & Phospho(ST) \\
\hline Q12873 & METEADAPSPAPSLGER & 2 Phospho(ST) \\
\hline Q12873 & ELQGDGPPSSPTNDPTVK & Phospho(ST) \\
\hline Q8IY81 & ALDISLSSGEEDEGDEEDSTAGTTK & 2 Phospho(ST) \\
\hline Q8IY81 & ALDISLSSGEEDEGDEEDSTAGTTK & 3 Phospho(ST) \\
\hline Q04721 & AEDEALLSEEDDPIDR & Phospho(ST) \\
\hline P62258 & AAFDDAIAELDTLSEESYK & Phospho(ST) \\
\hline Q92625 & SESLSNCSIGK & Phospho(ST) \\
\hline Q92625 & SPSFASEWDEIEK & Phospho(ST) \\
\hline Q9NVU0 & EAANEAGDSSQDEAEDDVK & 2 Phospho(ST) \\
\hline P08729 & SLDLDGIIAEVK & Phospho(ST) \\
\hline Q9H2P0 & LMHNASDSEVDQDDVVEWK & 2 Phospho(ST) \\
\hline Q92538 & AASSSSPGSPVASSPSR & Phospho(ST) \\
\hline
\end{tabular}




\begin{tabular}{|c|c|c|}
\hline Protein & Peptide sequence & Variable modification \\
\hline Q92538 & ADAPDAGAQSDSELPSYHQNDVSLDR & Phospho(ST) \\
\hline Q9HAU0 & GVISYQTLPR & Phospho(ST) \\
\hline Q9HAU0 & GLNVIGASDQSPLQSPSNLR & 2 Phospho(ST) \\
\hline Q9HAU0 & KGLNVIGASDQSPLQSPSNLR & 2 Phospho(ST) \\
\hline Q86WB0 & SQDATFSPGSEQAEK & Phospho(ST) \\
\hline P49023 & FIHQQPQSSSPVYGSSAK & Phospho(ST) \\
\hline P49023 & TGSSSPPGGPPKPGSQLDSMLGSLQSDLNK & Phospho(ST) \\
\hline Q96K21 & LPDSDDDEDEETAIQR & Phospho(ST) \\
\hline Q8NC44 & QALDSEEEEEDVAAK & Phospho(ST) \\
\hline Q8NC44 & $\begin{array}{l}\text { NAPPGGDEPLAETESESEAELAGFSPVVDV } \\
\mathrm{K}\end{array}$ & 2 Phospho(ST) \\
\hline Q9GZR7 & KAQAVSEEEEEEEGK & Phospho(ST) \\
\hline Q9GZR7 & AQAVSEEEEEEEGKSSSPK & 2 Phospho(ST) \\
\hline Q9GZR7 & $\begin{array}{l}\text { SPGKAEAESDALPDDTVIESEALPSDIAAEA } \\
\mathrm{R}\end{array}$ & Phospho(ST) \\
\hline P38432 & AFQLEEGEETEPDCK & Phospho(ST) \\
\hline Q96EZ8 & GDQVLNFSDAEDLIDDSK & Phospho(ST) \\
\hline Q1KMD3 & SKSPLPPEEEAK & Phospho(ST) \\
\hline Q1KMD3 & SGDETPGSEVPGDK & Phospho(ST) \\
\hline Q1KMD3 & EEDEPEERSGDETPGSEVPGDK & Phospho(ST) \\
\hline Q96N67 & SPSGSAFGSQENLR & Phospho(ST) \\
\hline P35251 & NKPLSPIK & Phospho(ST) \\
\hline P35251 & ESVSPEDSEK & Phospho(ST) \\
\hline P35251 & IIYDSDSESEETLQVK & 2 Phospho(ST) \\
\hline Q8WWM7 & STSTPTSPGPR & Phospho(ST) \\
\hline Q8WWM7 & EIESSPQYR & Phospho(ST) \\
\hline Q8WWM7 & LQPSSSPENSLDPFPPR & Phospho(ST) \\
\hline Q5XUX1 & TWDDDSDPESETDPDAQAK & Phospho(ST) \\
\hline Q92597 & SHTSEGTR & Phospho(ST) \\
\hline Q92597 & TASGSSVTSLDGTR & 2 Phospho(ST) \\
\hline Q92597 & TASGSSVTSLDGTR & 3 Phospho(ST) \\
\hline Q92597 & SRTASGSSVTSLDGTR & 4 Phospho(ST) \\
\hline Q92597 & SHTSEGAHLDITPNSGAAGNSAGPK & Phospho(ST) \\
\hline Q7Z2W4 & TVFSPTLPAAR & Phospho(ST) \\
\hline Q7Z2W4 & ASLEDAPVDDLTR & Phospho(ST) \\
\hline Q9Y2D5 & QVLQSTQSPR & Phospho(ST) \\
\hline Q9Y2D5 & DALGDSLQVPVSPSSTTSSR & Phospho(ST) \\
\hline Q9Y2D5 & GQKSPGALETPSAAGSQGNTASQGK & Phospho(ST) \\
\hline Q9BTC0 & TAAPSPSLLYK & Phospho(ST) \\
\hline Q9BTC0 & VKGGDDHDDTSDSDSDGLTLK & 3 Phospho(ST) \\
\hline Q9BTC0 & QEAIPDLEDSPPVSDSEEQQESAR & 2 Phospho(ST) \\
\hline Q9BTC0 & $\begin{array}{l}\text { KPSKYPLCSADAAVSTTPPGSPPPPPPLPEPP } \\
\text { VLK }\end{array}$ & 2 Phospho(ST) \\
\hline
\end{tabular}




\begin{tabular}{|c|c|c|}
\hline Protein & Peptide sequence & Variable modification \\
\hline P16949 & ESVPEFPLSPPK & Phospho(ST) \\
\hline P16949 & SKESVPEFPLSPPK & Phospho(ST) \\
\hline P16949 & ELEKRASGQAFELILSPR & 2 Phospho(ST) \\
\hline P04792 & GPSWDPFR & Phospho(ST) \\
\hline P04792 & QLSSGVSEIR & Phospho(ST) \\
\hline P04792 & AQLGGPEAAKSDETAAK & Phospho(ST) \\
\hline P23396 & DEILPTTPISEQK & Phospho(ST) \\
\hline Q96B36 & LNTSDFQK & Phospho(ST) \\
\hline Q96B36 & SLPVSVPVWGFK & Phospho(ST) \\
\hline Q96B36 & TEARSSDEENGPPSSPDLDR & 3 Phospho(ST) \\
\hline Q96B36 & AATAARPPAPPPAPQPPSPTPSPPRPTLAR & 2 Phospho(ST) \\
\hline Q99442 & KEETPGTPK & Phospho(ST) \\
\hline Q99442 & AESGKEEDKK & Phospho(ST) \\
\hline Q99442 & EELEQQTDGDCEEDEEEENDGETPK & Phospho(ST) \\
\hline O94876 & FGSADNIPNLK & Phospho(ST) \\
\hline O94876 & ALGVISNFQSSPK & Phospho(ST) \\
\hline O95425 & YQTQPVTLGEVEQVQSGK & Phospho(ST) \\
\hline Q9Y520 & STTPTSSPFR & Phospho(ST) \\
\hline Q9Y520 & LPDLSPVENK & Phospho(ST) \\
\hline Q9Y520 & $\begin{array}{l}\text { AAGSPSSSDQDEKLPGQDESTAGTSEQNDIL } \\
\mathrm{K}\end{array}$ & Phospho(ST) \\
\hline Q96EV2 & DIKEESDEEEEDDEESGR & Phospho(ST) \\
\hline Q96EV2 & AADEDWDSELEDDLLGEDLLSGK & Phospho(ST) \\
\hline Q9Y4E1 & RTPSDDEEDNLFAPPK & Phospho(ST) \\
\hline Q9Y4E1 & ERRTPSDDEEDNLFAPPK & 2 Phospho(ST) \\
\hline Q9Y4E1 & GLFSDEEDSEDLFSSQSASNLK & Phospho(ST) \\
\hline Q641Q2 & RTPSDDEEDNLFAPPK & Phospho(ST) \\
\hline Q641Q2 & GLFSDEEDSEDLFSSQSASK & Phospho(ST) \\
\hline Q641Q2 & ERRTPSDDEEDNLFAPPK & 2 Phospho(ST) \\
\hline Q641Q2 & ASALLFSSDEEDQWNIPASQTHLASDSR & 2 Phospho(ST) \\
\hline Q9BZE4 & TAAGEYDSVSESEDEEMLEIR & 3 Phospho(ST) \\
\hline Q8NHV4 & LVTSGAESGNLNTSPSSNQTR & Phospho(ST) \\
\hline Q8WX93 & SRDSGDENEPIQER & Phospho(ST) \\
\hline Q8WX93 & SRSRDSGDENEPIQER & 3 Phospho(ST) \\
\hline Q9NY27 & NHSDSSTSESEVSSVSPLK & Phospho(ST) \\
\hline Q7Z5L9 & KPSPEPEGEVGPPK & Phospho(ST) \\
\hline Q7Z5L9 & RKPSPEPEGEVGPPK & Phospho(ST) \\
\hline Q9Y6X9 & SPPLPAVIR & Phospho(ST) \\
\hline Q9Y6X9 & KDSNELSDSAGEEDSADLK & 2 Phospho(ST) \\
\hline Q14103 & NEEDEGHSNSSPR & Phospho(ST) \\
\hline Q14103 & IDASKNEEDEGHSNSSPR & Phospho(ST) \\
\hline Q13610 & EKLQEEGGGSDEEETGSPSEDGMQSAR & Phospho(ST) \\
\hline Q9UKM9 & GRLSPVPVPR & Phospho(ST) \\
\hline
\end{tabular}




\begin{tabular}{|c|c|c|}
\hline Protein & Peptide sequence & Variable modification \\
\hline Q9UKM9 & $\begin{array}{l}\text { DDGDEEGLLTHSEEELEHSQDTDADDGAL } \\
\text { Q }\end{array}$ & Phospho(ST) \\
\hline Q9UKM9 & $\begin{array}{l}\text { TRDDGDEEGLLTHSEEELEHSQDTDADDG } \\
\text { ALQ }\end{array}$ & Phospho(ST) \\
\hline Q9UKM9 & $\begin{array}{l}\text { TRDDGDEEGLLTHSEEELEHSQDTDADDG } \\
\text { ALQ }\end{array}$ & 2 Phospho(ST) \\
\hline Q14978 & RASSPFR & Phospho(ST) \\
\hline Q14978 & VADNSFDAK & Phospho(ST) \\
\hline Q14978 & LQTPNTFPK & Phospho(ST) \\
\hline Q14978 & GKGSPRPQAPK & Phospho(ST) \\
\hline Q14978 & AAKNSEEEEEEK & Phospho(ST) \\
\hline Q14978 & KKASSSDSEDSSEEEEEVQGPPAK & 6 Phospho(ST) \\
\hline Q5VZL5 & VTQHESDNENEIQIQNK & Phospho(ST) \\
\hline Q08J23 & AGEPNSPDAEEANSPDVTAGCDPAGVHPPR & Phospho(ST) \\
\hline Q08J23 & AGEPNSPDAEEANSPDVTAGCDPAGVHPPR & 2 Phospho(ST) \\
\hline P22466 & LLDLPAAASSEDIERS & Phospho(ST) \\
\hline O15027 & QIDSSPVGGETDETTVSQNYR & Phospho(ST) \\
\hline Q4KMP7 & ALAAGADSPK & Phospho(ST) \\
\hline Q4KMP7 & AAGGAPSPPPPVR & Phospho(ST) \\
\hline Q4KMP7 & QQPPLGPSSSLLSLPGLK & 2 Phospho(ST) \\
\hline Q4KMP7 & RQQPPLGPSSSLLSLPGLK & 2 Phospho(ST) \\
\hline P30050 & IGPLGLSPK & Phospho(ST) \\
\hline P55209 & EQSELDQDLDDVEEVEEEETGEETK & Phospho(ST) \\
\hline Q7Z6E9 & LEVTEIVKPSPK & Phospho(ST) \\
\hline Q7Z6E9 & WDKDDFESEEEDVK & Phospho(ST) \\
\hline Q5VSL9 & AASPPASASDLIEQQQK & Phospho(ST) \\
\hline P35611 & TPSFLKK & Phospho(ST) \\
\hline P35611 & SPGSPVGEGTGSPPK & Phospho(ST) \\
\hline P35611 & YSDVEVPASVTGYSFASDGDSGTCSPLR & Phospho(Y) \\
\hline P35612 & TPSFLKK & Phospho(ST) \\
\hline Q6KC79 & AITSLLGGGSPK & Phospho(ST) \\
\hline Q6KC79 & GSRPPLILQSQSLPCSSPR & Phospho(ST) \\
\hline Q6KC79 & YAEISSDEDNDSDEAFESSR & 2 Phospho(ST) \\
\hline Q8N7R7 & SFSADNFIGIQR & Phospho(ST) \\
\hline Q92508 & TASELLLDR & Phospho(ST) \\
\hline Q92508 & SGSEEAVTDPGER & Phospho(ST) \\
\hline Q14244 & LFVTPPEGSSR & Phospho(ST) \\
\hline Q14244 & LQLSPWESSVVNR & Phospho(ST) \\
\hline Q86VR2 & GQTPLTEGSEDLDGHSDPEESFAR & 4 Phospho(ST) \\
\hline Q86VR2 & AMDNHSDSEEELAAFCPQLDDSTVAR & 2 Phospho(ST) \\
\hline Q86VR2 & $\begin{array}{l}\text { SPSSDLDTDAEGDDFELLDQSELSQLDPASS } \\
\mathrm{R}\end{array}$ & 2 Phospho(ST) \\
\hline P51608 & AETSEGSGSAPAVPEASASPK & Phospho(ST) \\
\hline
\end{tabular}




\begin{tabular}{|c|c|c|}
\hline Protein & Peptide sequence & Variable modification \\
\hline Q9UNX4 & GSSPGIQDTLEAEDGAFETDEAPEDR & Phospho(ST) \\
\hline Q9UNX4 & IKGSSPGIQDTLEAEDGAFETDEAPEDR & Phospho(ST) \\
\hline Q99549 & GAEAFGDSEEDGEDVFEVEK & Phospho(ST) \\
\hline Q99549 & GYTSDDDTWEPEIHLEDCK & Phospho(ST) \\
\hline Q01831 & VIKDEALSDGDDLR & Phospho(ST) \\
\hline Q01831 & SEAAAPHTDAGGGLSSDEEEGTSSQAEAAR & 2 Phospho(ST) \\
\hline Q9Y618 & SLIGSPGR & Phospho(ST) \\
\hline Q9Y618 & SGLEPASSPSK & Phospho(ST) \\
\hline Q9Y618 & EGTPPPPPPSR & Phospho(ST) \\
\hline Q9Y618 & ANASPQKPLDLK & Phospho(ST) \\
\hline Q9Y618 & LEPVSPPSPPHTDPELELVPPR & 2 Phospho(ST) \\
\hline Q9Y618 & SLGYHGSSYSPEGVEPVSPVSSPSLTHDK & 2 Phospho(ST) \\
\hline O95361 & ETEEQDSDSAEQGDPAGEGK & Phospho(ST) \\
\hline Q13263 & $\begin{array}{l}\text { STAPSAAASASASAAASSPAGGGAEALELL } \\
\text { EHCGVCR }\end{array}$ & Phospho(ST) \\
\hline Q13263 & $\begin{array}{l}\text { LASPSGSTSSGLEVVAPEGTSAPGGGPGTLD } \\
\text { DSATICR }\end{array}$ & Phospho(ST) \\
\hline P14618 & LDIDSPPITAR & Phospho(ST) \\
\hline Q16643 & LSSPVLHR & Phospho(ST) \\
\hline Q16643 & SPSDSSTASTPVAEQIER & Phospho(ST) \\
\hline Q16643 & MAPTPIPTRSPSDSSTASTPVAEQIER & Phospho(ST) \\
\hline Q8NAV1 & VPSPDHR & Phospho(ST) \\
\hline Q8NAV1 & RRSPSPR & 2 Phospho(ST) \\
\hline Q8NAV1 & VSALEEDMDDVESSEEEEEEDEKLER & 2 Phospho(ST) \\
\hline P21291 & GFGFGQGAGALVHSE & Phospho(ST) \\
\hline Q14676 & LLLAEDSEEEVDFLSER & Phospho(ST) \\
\hline Q14676 & AQPFGFIDSDTDAEEER & 2 Phospho(ST) \\
\hline Q96T58 & LNTVASPK & Phospho(ST) \\
\hline Q96T58 & NVDAAVSPR & Phospho(ST) \\
\hline Q96T58 & RPQSPGASPSQAER & 2 Phospho(ST) \\
\hline Q96T58 & SESPQKEDGLSSQLK & Phospho(ST) \\
\hline Q96T58 & DLEPGEVPSDSDEDGEHK & 2 Phospho(ST) \\
\hline Q96T58 & EVSGFRGGGGGPAYGPPPSLHAR & Phospho(ST) \\
\hline Q96T58 & SLVHEVGKPPQDVTDDSPPSK & Phospho(ST) \\
\hline Q96T58 & ESGVVAVSPEKSESPQKEDGLSSQLK & 2 Phospho(ST) \\
\hline Q9H0D6 & AEDSDSEPEPEDNVR & 2 Phospho(ST) \\
\hline Q9H0D6 & KAEDSDSEPEPEDNVR & 2 Phospho(ST) \\
\hline P49006 & LSGLSFK & Phospho(ST) \\
\hline P49006 & AAATPESQEPQAK & Phospho(ST) \\
\hline P49006 & GDVTAEEAAGASPAK & Phospho(ST) \\
\hline P12270 & EGVQGPLNVSLSEEGK & Phospho(ST) \\
\hline P12270 & TDGFAEAIHSPQVAGVPR & Phospho(ST) \\
\hline O00499 & SPSPPDGSPAATPEIR & Phospho(ST) \\
\hline
\end{tabular}




\begin{tabular}{|c|c|c|}
\hline Protein & Peptide sequence & Variable modification \\
\hline O00499 & GNKSPSPPDGSPAATPEIR & Phospho(ST) \\
\hline O00499 & VNHEPEPAGGATPGATLPKSPSQLR & Phospho(ST) \\
\hline Q08945 & IKSDHPGISITDLSK & 2 Phospho(ST) \\
\hline Q08945 & SKEFVSSDESSSGENK & 4 Phospho(ST) \\
\hline Q08945 & EGMNPSYDEYADSDEDQHDAYLER & Phospho(ST) \\
\hline Q8TBB5 & SEDEDSLEEAGSPAPGPCPR & 2 Phospho(ST) \\
\hline Q8TBB5 & $\begin{array}{l}\text { QVLTAPGSAGQPRSEDEDSLEEAGSPAPGP } \\
\text { CPR }\end{array}$ & 2 Phospho(ST) \\
\hline Q1ED39 & YSVLNNDDYFADVSPLR & Phospho(ST) \\
\hline Q13112 & TQDPSSPGTTPPQAR & Phospho(ST) \\
\hline Q13112 & GSSPGPRPVEGTPASR & Phospho(ST) \\
\hline O14639 & QSLGESPR & Phospho(ST) \\
\hline O14639 & TLSPTPSAEGYQDVR & Phospho(ST) \\
\hline Q9Y232 & TAVDGFQSESPEK & Phospho(ST) \\
\hline Q9Y232 & $\begin{array}{l}\text { TAVDGFQSESPEKLDPVEQGQEDTVAPEVA } \\
\text { AEKPVGALLGPGAER }\end{array}$ & Phospho(ST) \\
\hline Q13425 & GLGPPSPPAPPR & Phospho(ST) \\
\hline Q13425 & GPAGEAGASPPVR & Phospho(ST) \\
\hline Q13425 & GPAGEAGASPPVRR & Phospho(ST) \\
\hline Q9BUH6 & LAAAEETAVSPR & Phospho(ST) \\
\hline Q9NPQ8 & GLMAGGRPEGQYSEDEDTDTDEYK & 2 Phospho(ST) \\
\hline P78316 & HNDIVDSDSDAEDR & 2 Phospho(ST) \\
\hline Q2KHR2 & NLSGSTLYPVSNIPR & Phospho(ST) \\
\hline Q13247 & LIVENLSSR & Phospho(ST) \\
\hline Q13247 & SNSPLPVPPSK & Phospho(ST) \\
\hline Q13247 & ARSVSPPPKR & 2 Phospho(ST) \\
\hline Q13243 & SKSPASVDR & Phospho(ST) \\
\hline Q13243 & LIVENLSSR & Phospho(ST) \\
\hline Q08170 & SLRQSR & Phospho(ST) \\
\hline Q08170 & LIVENLSSR & Phospho(ST) \\
\hline P10644 & EDEISPPPPNPVVK & Phospho(ST) \\
\hline P10644 & TDSREDEISPPPPNPVVK & Phospho(ST) \\
\hline Q16513 & ASSLGEIDESSELR & Phospho(ST) \\
\hline Q16513 & ATSVALPGWSPSETR & Phospho(ST) \\
\hline Q9NX40 & LENSPLGEALR & Phospho(ST) \\
\hline Q9NX40 & KLENSPLGEALR & Phospho(ST) \\
\hline P52292 & NVSSFPDDATSPLQENR & Phospho(ST) \\
\hline P11717 & LVSFHDDSDEDLLHI & Phospho(ST) \\
\hline P11717 & ALSSLHGDDQDSEDEVLTIPEVK & Phospho(ST) \\
\hline Q9Y446 & LSSGFDDIDLPSAVK & Phospho(ST) \\
\hline Q9UH62 & YNDWSDDDDDSNESK & Phospho(ST) \\
\hline O43294 & KRPSLPSSPSPGLPK & Phospho(ST) \\
\hline O43294 & KRPSLPSSPSPGLPK & 2 Phospho(ST) \\
\hline
\end{tabular}




\begin{tabular}{|c|c|c|}
\hline Protein & Peptide sequence & Variable modification \\
\hline O43294 & SPKPAAPAAPPFSSSSGVLGTGLCELDR & Phospho(ST) \\
\hline Q969X1 & AVSDSFGPGEWDDR & Phospho(ST) \\
\hline P62805 & RISGLIYEETR & Phospho(ST) \\
\hline Q9UDY2 & GSYGSDAEEEEYR & Phospho(ST) \\
\hline P22059 & MLAESDESGDEESVSQTDK & 2 Phospho(ST) \\
\hline P22059 & GDMSDEDDENEFFDAPEIITMPENLGHK & Phospho(ST) \\
\hline Q9ULF5 & LNETELTDLEGQQESPPK & Phospho(ST) \\
\hline Q9BW71 & LGSTSGEESDLER & 3 Phospho(ST) \\
\hline Q9BW71 & LGSTSGEESDLER & 4 Phospho(ST) \\
\hline Q9BW71 & NGVAAEVSPAKEENPR & Phospho(ST) \\
\hline Q9BW71 & SLKESEQESEEEILAQK & 2 Phospho(ST) \\
\hline Q9BW71 & RLSGSSEDEEDSGKGEPTAK & 3 Phospho(ST) \\
\hline O95365 & GGAPDPSPGATATPGAPAQPSSPDAR & Phospho(ST) \\
\hline O14745 & EALAEAALESPRPALVR & Phospho(ST) \\
\hline O14745 & SASSDTSEELNSQDSPPK & Phospho(ST) \\
\hline P21796 & LTFDSSFSPNTGK & Phospho(ST) \\
\hline Q9H0G5 & VEENPDADSDFDAKSSADDEIEETR & 3 Phospho(ST) \\
\hline Q9H0G5 & $\begin{array}{l}\text { KTQQLHPVLQKPSVFGNDSDDDDETSVSES } \\
\text { LQR }\end{array}$ & Phospho(ST) \\
\hline Q5JTH9 & GDSIEEILADSEDEEDNEEEER & Phospho(ST) \\
\hline O60583 & QGQSQAASSSSVTSPIK & Phospho(ST) \\
\hline Q86VM9 & RPNTSPDR & Phospho(ST) \\
\hline Q86VM9 & ASDLEDEESAAR & Phospho(ST) \\
\hline Q8WUF5 & DNLTSATLPR & Phospho(ST) \\
\hline Q9H788 & TLSSSAQEDIIR & Phospho(ST) \\
\hline O15541 & YGVYEDENYEVGSDDEEIPFK & Phospho(ST) \\
\hline O15541 & AAYGDLSSEEEEENEPESLGVVYK & 2 Phospho(ST) \\
\hline O15541 & AAYGDLSSEEEEENEPESLGVVYK & Phospho(ST) \\
\hline Q9BXB4 & SFSLASSSNSPISQR & Phospho(ST) \\
\hline Q9BXB4 & SFSLASSSNSPISQR & 2 Phospho(ST) \\
\hline Q66PJ3 & SAGEEEDGPVLTDEQK & Phospho(ST) \\
\hline Q5H9R7 & IQQFDDGGSDEEDIWEEK & Phospho(ST) \\
\hline Q9H0H5 & SIGSAVDQGNESIVAK & Phospho(ST) \\
\hline Q9H0H5 & SIGSAVDQGNESIVAK & 2 Phospho(ST) \\
\hline O43432 & RSPVPAQIAITVPK & Phospho(ST) \\
\hline $\mathrm{O} 43432$ & LDFIESDSPCSSEALSK & Phospho(ST) \\
\hline Q8TAQ2 & KRSPSPSPTPEAK & 2 Phospho(ST) \\
\hline Q8TAQ2 & TLTDEVNSPDSDRR & Phospho(ST) \\
\hline Q8WWQ0 & VLSDSEDEEKDADVPGTSTR & 2 Phospho(ST) \\
\hline Q8WWQ0 & KVLSDSEDEEKDADVPGTSTR & 2 Phospho(ST) \\
\hline O60763 & DLGHPVEEEDELESGDQEDEDDESEDPGK & Phospho(ST) \\
\hline O60763 & $\begin{array}{l}\text { DLGHPVEEEDELESGDQEDEDDESEDPGKD } \\
\text { LDHI }\end{array}$ & Phospho(ST) \\
\hline
\end{tabular}




\begin{tabular}{|c|c|c|}
\hline Protein & Peptide sequence & Variable modification \\
\hline Q14684 & GSPTGGAQLLK & Phospho(ST) \\
\hline Q14684 & VGDGDLSAEEIPENEVSLR & Phospho(ST) \\
\hline P53396 & AKPAMPQDSVPSPR & Phospho(ST) \\
\hline Q9BPX3 & TLHCEGTEINSDDEQESK & Phospho(ST) \\
\hline Q00587 & RSDSLLSFR & Phospho(ST) \\
\hline Q00587 & ASWESLDEEWR & Phospho(ST) \\
\hline Q00341 & VATLNSEEESDPPTYK & Phospho(ST) \\
\hline Q7L2J0 & TLNAETPKSSPLPAK & Phospho(ST) \\
\hline Q7L2J0 & TLNAETPKSSPLPAK & 2 Phospho(ST) \\
\hline Q7L2J0 & ESPGAAATSSSGPQAQQHR & Phospho(ST) \\
\hline Q7L2J0 & $\begin{array}{l}\text { DEVVSPLPSALQGPSGSLSAPPAASVISAPPS } \\
\text { SSSR }\end{array}$ & Phospho(ST) \\
\hline Q13620 & SATDGNTSTTPPTSAK & Phospho(ST) \\
\hline Q9NRY4 & TSFSVGSDDELGPIR & Phospho(ST) \\
\hline A2RRP1 & AGEEDEGEEDSDSDYEISAK & Phospho(ST) \\
\hline Q9Y388 & EVQAEQPSSSSPR & Phospho(ST) \\
\hline Q15477 & ASSLEDLVLK & Phospho(ST) \\
\hline O60341 & EMDESLANLSEDEYYSEEER & Phospho(ST) \\
\hline Q96E09 & HGLLLPASPVR & Phospho(ST) \\
\hline Q96E09 & IDFIPVSPAPSPTR & 2 Phospho(ST) \\
\hline Q96E09 & SNSAPLIHGLSDTSPVFQAEAPSAR & Phospho(ST) \\
\hline O15446 & QEQINTEPLEDTVLSPTK & Phospho(ST) \\
\hline Q9HCN4 & GTLDEEDEEADSDTDDIDHR & Phospho(ST) \\
\hline O60504 & LCDDGPQLPTSPR & Phospho(ST) \\
\hline Q9NXG2 & FTDKDQQPSGSEGEDDDAEAALK & 2 Phospho(ST) \\
\hline Q9NXG2 & FTDKDQQPSGSEGEDDDAEAALKK & 2 Phospho(ST) \\
\hline Q9NYB0 & YLLGDAPVSPSSQK & Phospho(ST) \\
\hline Q6P158 & DLQEQDADAGSER & Phospho(ST) \\
\hline Q6P158 & GLSGEEEDDEPDCCNDER & Phospho(ST) \\
\hline Q9NTI5 & TPSPSQPK & Phospho(ST) \\
\hline Q9NTI5 & LKEDILENEDEQNSPPK & Phospho(ST) \\
\hline Q9NTI5 & LKEDILENEDEQNSPPKK & Phospho(ST) \\
\hline Q96F86 & SQDVAVSPQQQQCSK & Phospho(ST) \\
\hline Q12789 & NSSTDQGSDEEGSLQK & Phospho(ST) \\
\hline Q8N7H5 & DKEEIFGSDADSEDDADSDDEDR & 3 Phospho(ST) \\
\hline P49321 & KPEEESPR & Phospho(ST) \\
\hline P49321 & LVPSQEETK & Phospho(ST) \\
\hline P49321 & VAQGATEKSPEDK & Phospho(ST) \\
\hline P49321 & EGEETEGSEEDDKENDKTEEMPNDSVLENK & 2 Phospho(ST) \\
\hline P34947 & DVLDIEQFSTVK & Phospho(ST) \\
\hline Q96QR8 & RGGGSGGGEESEGEEVDED & Phospho(ST) \\
\hline O60293 & SVVVTLNDSDDSESDGEASK & 3 Phospho(ST) \\
\hline O60293 & KPISDNSFSSDEEQSTGPIK & 2 Phospho(ST) \\
\hline
\end{tabular}




\begin{tabular}{|c|c|c|}
\hline Protein & Peptide sequence & Variable modification \\
\hline O60293 & EEGELEDGEISDDDNNSQIR & Phospho(ST) \\
\hline O94906 & LSQVSDSVSGQTVVDPK & Phospho(ST) \\
\hline Q8IVT2 & ALSSDSILSPAPDAR & 2 Phospho(ST) \\
\hline Q8IVT2 & RALSSDSILSPAPDAR & Phospho(ST) \\
\hline Q8IVT2 & ASTPDWVSEGPQPGLR & Phospho(ST) \\
\hline Q53H80 & TLDFDPLLSPASPK & Phospho(ST) \\
\hline Q3MHD2 & TETPPPLASLNVSK & Phospho(ST) \\
\hline Q13547 & IACEEEFSDSEEEGEGGR & 2 Phospho(ST) \\
\hline Q13547 & IACEEEFSDSEEEGEGGRK & 2 Phospho(ST) \\
\hline O15534 & GLDSSSTAPSALGER & Phospho(ST) \\
\hline O75970 & DADLSPVNASIIK & Phospho(ST) \\
\hline O00566 & KSPVFSDEDSDLDFDISK & 3 Phospho(ST) \\
\hline O00566 & SDLRKSPVFSDEDSDLDFDISK & 3 Phospho(ST) \\
\hline Q13823 & DDAEESSSEPEEENVGNDTK & 2 Phospho(ST) \\
\hline Q13823 & DDAEESSSEPEEENVGNDTK & 3 Phospho(ST) \\
\hline P05114 & TEESPASDEAGEK & Phospho(ST) \\
\hline Q3KQU3 & LSASTASELSPK & Phospho(ST) \\
\hline Q3KQU3 & SSQPSPTAVPASDSPPTK & Phospho(ST) \\
\hline Q3KQU3 & ESAAPASPAPSPAPSPTPAPPQK & Phospho(ST) \\
\hline Q3KQU3 & ESAAPASPAPSPAPSPTPAPPQK & 3 Phospho(ST) \\
\hline P60174 & KQSLGELIGTLNAAK & Phospho(ST) \\
\hline Q8TEA8 & SASSGAEGDVSSEREP & Phospho(ST) \\
\hline Q8TEA8 & SASSGAEGDVSSEREP & 2 Phospho(ST) \\
\hline Q53EL6 & FVSEGDGGR & Phospho(ST) \\
\hline Q53EL6 & SGLTVPTSPK & Phospho(ST) \\
\hline Q53EL6 & DSGRGDSVSDSGSDALR & 2 Phospho(ST) \\
\hline Q9Y383 & SRSHQR & Phospho(ST) \\
\hline Q9Y383 & DQDLASCDR & Phospho(ST) \\
\hline Q9Y383 & AMLDQLMGTSR & Phospho(ST) \\
\hline Q9Y383 & FRDQDLASCDR & Phospho(ST) \\
\hline P59510 & KDSHQR & Phospho(ST) \\
\hline Q9UK13 & RSSHQR & Phospho(ST) \\
\hline Q86WC4 & SSTSFANIQENSN & Phospho(ST) \\
\hline Q9Y2F5 & SCSSPAVSAVSQLPLSPK & 2 Phospho(ST) \\
\hline O00505 & NVPQEESLEDSDVDADFK & Phospho(ST) \\
\hline Q9UHD8 & SFEVEEVETPNSTPPR & Phospho(ST) \\
\hline Q9UER7 & TSVATQCDPEEIIVLSDSD & 2 Phospho(ST) \\
\hline O43399 & NSATFKSFEDR & Phospho(ST) \\
\hline O43399 & GLLSDSMTDVPVDTGVAAR & Phospho(ST) \\
\hline Q13523 & KSPIINESR & Phospho(ST) \\
\hline Q13523 & KKSPIINESR & Phospho(ST) \\
\hline Q13523 & VQSGMGLILQGYESGSEEEGEIHEK & 2 Phospho(ST) \\
\hline Q13523 & VEQESSSDDNLEDFDVEEEDEEALIEQR & 3 Phospho(ST) \\
\hline
\end{tabular}




\begin{tabular}{|c|c|c|}
\hline Protein & Peptide sequence & Variable modification \\
\hline P35606 & STAQQELDGKPASPTPVIVASHTANK & Phospho(ST) \\
\hline P35606 & STAQQELDGKPASPTPVIVASHTANKEEK & Phospho(ST) \\
\hline $\mathrm{O} 43237$ & DFQDYMEPEEGCQGSPQR & Phospho(ST) \\
\hline Q8N556 & TGSNAAQYK & Phospho(ST) \\
\hline Q8N556 & EAYSGCSGPVDSECPPPPSSPVHK & Phospho(ST) \\
\hline Q01850 & SSSETILSSLAGSDIVK & Phospho(ST) \\
\hline Q92922 & KHSPSPPPPTPTESR & 2 Phospho(ST) \\
\hline Q13045 & NAEAVLQSPGLSGK & Phospho(ST) \\
\hline Q14432 & ISPLSSPCSSPLQGTPASSLVSK & Phospho(ST) \\
\hline Q14432 & VNPVTSLSENYTCSDSEESSEK & Phospho(ST) \\
\hline Q14432 & VNPVTSLSENYTCSDSEESSEKDK & Phospho(ST) \\
\hline Q8IXM2 & KVYEDSGIPLPAESPK & Phospho(ST) \\
\hline Q9NWV8 & SEGEGEAASADDGSLNTSGAGPK & Phospho(ST) \\
\hline Q14160 & MKSLEQDALR & Phospho(ST) \\
\hline Q14160 & LPLLPPESPGPLR & Phospho(ST) \\
\hline Q14160 & RSEACPCQPDSGSPLPAEEEK & Phospho(ST) \\
\hline Q14160 & $\begin{array}{l}\text { LAEAPSPAPTPSPTPVEDLGPQTSTSPGRLSP } \\
\text { DFAEELR }\end{array}$ & 2 Phospho(ST) \\
\hline Q96G74 & $\begin{array}{l}\text { QAPGVGAVGGGSPEREEVGAGYNSEDEYE } \\
\text { AAAAR }\end{array}$ & 2 Phospho(ST) \\
\hline O94880 & NSADDEELTNDSLTLSQSK & 2 Phospho(ST) \\
\hline Q15527 & DLGSTEDGDGTDDFLTDKEDEK & 2 Phospho(ST) \\
\hline Q15527 & KDLGSTEDGDGTDDFLTDKEDEK & 2 Phospho(ST) \\
\hline Q96PK6 & TRLSPPR & Phospho(ST) \\
\hline Q96PK6 & QPTPPFFGR & Phospho(ST) \\
\hline Q96PK6 & LSESQLSFR & Phospho(ST) \\
\hline Q96PK6 & AQPSASLGVGYR & Phospho(ST) \\
\hline B5ME19 & QNPEQSADEDAEK & Phospho(ST) \\
\hline B5ME19 & QPLLLSEDEEDTK & Phospho(ST) \\
\hline O00418 & LEGVDGGQSPR & Phospho(ST) \\
\hline O00418 & KYESDEDSLGSSGR & Phospho(ST) \\
\hline P00558 & ALESPERPFLAILGGAK & Phospho(ST) \\
\hline Q6NXT2 & STELLIRK & Phospho(ST) \\
\hline P33527 & QLSSSSSYSGDISR & 2 Phospho(ST) \\
\hline P50502 & ADEPSSEESDLEIDK & 2 Phospho(ST) \\
\hline P50502 & KVEEDLKADEPSSEESDLEIDK & 3 Phospho(ST) \\
\hline Q8IZP2 & ADEPSSEESDLEIDK & 2 Phospho(ST) \\
\hline Q8N1G4 & EEGSLSDTEADAVSGQLPDPTTNPSAGK & Phospho(ST) \\
\hline Q8N1G4 & $\begin{array}{l}\text { YTLENKEEGSLSDTEADAVSGQLPDPTTNP } \\
\text { SAGK }\end{array}$ & Phospho(ST) \\
\hline Q68EM7 & SPSPPTQHTGQPPGQPSAPSQLSAPR & Phospho(ST) \\
\hline Q68EM7 & SPSPPTQHTGQPPGQPSAPSQLSAPR & 2 Phospho(ST) \\
\hline Q68EM7 & AESSSGGGTVPSSAGILEQGPSPGDGSPPKP & Phospho(ST) \\
\hline
\end{tabular}




\begin{tabular}{|c|c|c|}
\hline Protein & Peptide sequence & Variable modification \\
\hline & $\mathrm{K}$ & \\
\hline Q9Y6D5 & GQSQLSNPTDDSWK & Phospho(ST) \\
\hline Q9Y6D5 & ELEKPIQSKPQSPVIQAAAVSPK & Phospho(ST) \\
\hline Q00526 & IGEGTYGVVYK & Phospho(ST) \\
\hline O95218 & EVEDKESEGEEEDEDEDLSK & Phospho(ST) \\
\hline O95218 & ENVEYIEREESDGEYDEFGR & Phospho(ST) \\
\hline O95218 & YKLDEDEDEDDADLSKYNLDASEEEDSNK & Phospho(ST) \\
\hline Q92522 & AGGSAALSPSK & Phospho(ST) \\
\hline Q29RF7 & AAVGQESPGGLEAGNAK & Phospho(ST) \\
\hline Q96AP0 & ELILGSETPSSPR & Phospho(ST) \\
\hline P52701 & SEEDNEIESEEEVQPK & Phospho(ST) \\
\hline O60832 & AKEVELVSE & Phospho(ST) \\
\hline O60832 & AGLESGAEPGDGDSDTTKK & Phospho(ST) \\
\hline O60832 & KRESESESDETPPAAPQLIK & 2 Phospho(ST) \\
\hline O60832 & KRESESESDETPPAAPQLIK & 3 Phospho(ST) \\
\hline P49916 & LTTTGQVTSPVK & Phospho(ST) \\
\hline P49840 & GEPNVSYICSR & Phospho(Y) \\
\hline Q96K76 & STETSDFENIESPLNER & Phospho(ST) \\
\hline Q9H3Z4 & SLSTSGESLYHVLGLDK & Phospho(ST) \\
\hline Q8TAD8 & QERLSPEVAPPAHR & Phospho(ST) \\
\hline Q8TAD8 & DDEDEEEEEEVSDS & Phospho(ST) \\
\hline Q8TAD8 & KDDEDEEEEEEVSDS & Phospho(ST) \\
\hline Q8TAD8 & RPDHSGGSPSPPTSEPAR & Phospho(ST) \\
\hline Q5UIP0 & ASQGLLSSIENSESDSSEAK & Phospho(ST) \\
\hline Q12906 & $\begin{array}{l}\text { DSSKGEDSAEETEAKPAVVAPAPVVEAVST } \\
\text { PSAAFPSDATAEQGPILTK }\end{array}$ & Phospho(ST) \\
\hline Q6Y7W6 & SQSWEER & Phospho(ST) \\
\hline Q6Y7W6 & ALSSGGSITSPPLSPALPK & Phospho(ST) \\
\hline Q9Y5X1 & SGNWESSEGWGAQPEGAGAQR & Phospho(ST) \\
\hline Q13439 & EENPESDGEPVVEDGTSVK & Phospho(ST) \\
\hline O75175 & STDSEVSQSPAK & Phospho(ST) \\
\hline Q14669 & AQTAPTKTSPR & Phospho(ST) \\
\hline Q14669 & DDSLDLSPQGR & Phospho(ST) \\
\hline Q14669 & SESPPAELPSLR & Phospho(ST) \\
\hline Q8N122 & VLDTSSLTQSAPASPTNK & 2 Phospho(ST) \\
\hline Q9BRD0 & HGTPDPSPR & 2 Phospho(ST) \\
\hline Q9BRD0 & VHNNSPDTSR & Phospho(ST) \\
\hline Q15434 & GYGFVDFDSPSAAQK & Phospho(ST) \\
\hline Q8NEF9 & EYFDDSTEER & Phospho(ST) \\
\hline Q8NEF9 & AVTIANSPSKPSEK & Phospho(ST) \\
\hline Q13098 & EGSQGELTPANSQSR & Phospho(ST) \\
\hline Q96S94 & GLLPGGTQVLDGTSGFSPAPK & Phospho(ST) \\
\hline Q9BUH8 & AGGDLSLSPGR & Phospho(ST) \\
\hline
\end{tabular}




\begin{tabular}{|c|c|c|}
\hline Protein & Peptide sequence & Variable modification \\
\hline Q9BUH8 & DRRPSVDAPVTDVGFLR & Phospho(ST) \\
\hline O75153 & SEDPPGQEAGSEEEGSSASGLAK & Phospho(ST) \\
\hline Q8TCJ2 & ENPPVEDSSDEDDKR & Phospho(ST) \\
\hline Q8TCJ2 & ENPPVEDSSDEDDKR & 2 Phospho(ST) \\
\hline Q9NQS1 & $\begin{array}{l}\text { REPGGWGAGASAPVEDDSDAETYGEENDE } \\
\text { QGNYSK }\end{array}$ & Phospho(ST) \\
\hline P17612 & TWTLCGTPEYLAPEIILSK & Phospho(ST) \\
\hline Q9H8Y8 & VGDSTPVSEKPVSAAVDANASESP & Phospho(ST) \\
\hline Q86YP4 & RPPSPDVIVLSDNEQPSSPR & 2 Phospho(ST) \\
\hline Q86YP4 & RPPSPDVIVLSDNEQPSSPR & 3 Phospho(ST) \\
\hline Q86VQ1 & SASWGSADQLK & Phospho(ST) \\
\hline Q86VQ1 & DKDSGSSSPLPK & 2 Phospho(ST) \\
\hline Q86VQ1 & SIDTQTPSVQER & 2 Phospho(ST) \\
\hline Q9UGU0 & AGSSPAQGAQNEPPR & Phospho(ST) \\
\hline P49815 & SSSSPELQTLQDILGDPGDK & Phospho(ST) \\
\hline P60468 & PGPTPSGTNVGSSGRSPSK & Phospho(ST) \\
\hline P60468 & PGPTPSGTNVGSSGRSPSK & 2 Phospho(ST) \\
\hline Q9Y3X0 & EGAASPAPETPQPTSPETSPK & Phospho(ST) \\
\hline Q9Y3X0 & EGAASPAPETPQPTSPETSPK & 2 Phospho(ST) \\
\hline P58107 & QVSASELHTSGILGPETLR & Phospho(ST) \\
\hline P58107 & RQVSASELHTSGILGPETLR & Phospho(ST) \\
\hline Q96T37 & DGWSLDR & Phospho(ST) \\
\hline Q96T37 & DRTPPLLYR & Phospho(ST) \\
\hline Q96T37 & LLLERPSPIR & Phospho(ST) \\
\hline Q96T37 & SLSPGGAALGYR & 2 Phospho(ST) \\
\hline Q96T37 & SRSPLDKDTYPPSASVVGASVGGHR & Phospho(ST) \\
\hline Q96T37 & SRSPLDKDTYPPSASVVGASVGGHR & 2 Phospho(ST) \\
\hline Q9UQE7 & GSGSQSSVPSVDQFTGVGIR & Phospho(ST) \\
\hline Q9UQE7 & KGDVEGSQSQDEGEGSGESER & Phospho(ST) \\
\hline Q6SPF0 & GATPPAPPR & Phospho(ST) \\
\hline Q6SPF0 & AAAAAATAPPSPGPAQPGPR & Phospho(ST) \\
\hline Q96GN5 & EVADIFNAPSDDEEFVGFR & Phospho(ST) \\
\hline Q96IZ0 & RSTGVVNIPAAECLDEYEDDEAGQK & Phospho(ST) \\
\hline Q07666 & SGSMDPSGAHPSVR & Phospho(ST) \\
\hline Q9Y2D8 & VDDSTGTVISDVEEDAGELSR & Phospho(ST) \\
\hline O75128 & VSLGSQIDLQK & Phospho(ST) \\
\hline P31943 & HTGPNSPDTANDGFVR & Phospho(ST) \\
\hline Q96NB3 & KEEENADSDDEGELQDLLSQDWR & Phospho(ST) \\
\hline Q13177 & YLSFTPPEK & Phospho(ST) \\
\hline O95747 & TAQALSSGSGSQETK & Phospho(ST) \\
\hline O95747 & TEDGGWEWSDDEFDEESEEGK & Phospho(ST) \\
\hline Q96IF1 & SSFASSSASDASKPSSPR & Phospho(ST) \\
\hline Q9Y2V2 & GNVVPSPLPTR & Phospho(ST) \\
\hline
\end{tabular}




\begin{tabular}{|c|c|c|}
\hline Protein & Peptide sequence & Variable modification \\
\hline Q9Y2V2 & ERSPSPLRGNVVPSPLPTR & 3 Phospho(ST) \\
\hline Q16555 & GLYDGPVCEVSVTPK & Phospho(ST) \\
\hline Q99733 & LDNVPHTPSSYIETLPK & Phospho(ST) \\
\hline Q99733 & EFITGDVEPTDAESEWHSENEEEEK & Phospho(ST) \\
\hline O75151 & DSDYVYPSLESDEDNPIFK & Phospho(ST) \\
\hline Q9UKV3 & SLSPGVSR & Phospho(ST) \\
\hline Q9UKV3 & STPVRDR & 2 Phospho(ST) \\
\hline Q9UKV3 & TAQVPSPPR & Phospho(ST) \\
\hline Q9UKV3 & SSSISEEKGDSDDEKPR & Phospho(ST) \\
\hline Q9UKV3 & ASLVALPEQTASEEETPPPLLTK & Phospho(ST) \\
\hline Q9UKV3 & ASLVALPEQTASEEETPPPLLTK & 2 Phospho(ST) \\
\hline O75694 & AAPQSPSVPK & Phospho(ST) \\
\hline P49454 & LALSPLSLGK & Phospho(ST) \\
\hline P49454 & LQLQGLDLSSR & Phospho(ST) \\
\hline Q9H5H4 & TQSPEFEAQSSK & Phospho(ST) \\
\hline Q9H5H4 & SPGLVPPSPEFAPR & 2 Phospho(ST) \\
\hline Q01105 & RQSPLPPQK & Phospho(ST) \\
\hline Q01105 & RQSPLPPQKK & Phospho(ST) \\
\hline O60271 & SSTLSQLPGDK & Phospho(ST) \\
\hline O60271 & SASQSSLDKLDQELK & Phospho(ST) \\
\hline O60271 & SASQSSLDKLDQELK & 2 Phospho(ST) \\
\hline O60271 & LHQLSGSDQLESTAHSR & Phospho(ST) \\
\hline Q14C86 & SSDIVSSVR & Phospho(ST) \\
\hline Q14C86 & SRSSDIVSSVR & Phospho(ST) \\
\hline Q14C86 & EVSSRPSTPGLSVVSGISATSEDIPNK & 2 Phospho(ST) \\
\hline P52948 & SLVGGLLQSK & Phospho(ST) \\
\hline P52948 & YGLQDSDEEEEEHPSK & Phospho(ST) \\
\hline O95239 & TFSLTEVR & Phospho(ST) \\
\hline Q06265 & APIDTSDVEEK & Phospho(ST) \\
\hline Q9UPT8 & TGSGSPFAGNSPAR & Phospho(ST) \\
\hline Q9UPT8 & AAKPGPAEAPSPTASPSGDASPPATAPYDPR & Phospho(ST) \\
\hline Q12982 & KGSITEYTAAEEK & Phospho(ST) \\
\hline O95248 & LGLGTLSSSLSR & Phospho(ST) \\
\hline O75367 & GKLEAIITPPPAK & Phospho(ST) \\
\hline Q8IU81 & SPGPPALK & Phospho(ST) \\
\hline Q8IU81 & NVAEALGHSPK & Phospho(ST) \\
\hline Q8IU81 & AGGASPAASSTAQPPTQHR & Phospho(ST) \\
\hline O75351 & GNDSDGEGESDDPEKK & Phospho(ST) \\
\hline O75351 & EGQPSPADEKGNDSDGEGESDDPEKK & Phospho(ST) \\
\hline P17029 & EATGLSPQAAQEK & Phospho(ST) \\
\hline Q9UPU5 & VSDQNSPVLPK & Phospho(ST) \\
\hline Q9UPU5 & TLLSESSSQSSKSPSLSSK & Phospho(ST) \\
\hline Q9ULX3 & KDDSDDDGGGWITPSNIK & Phospho(ST) \\
\hline
\end{tabular}




\begin{tabular}{|c|c|c|}
\hline Protein & Peptide sequence & Variable modification \\
\hline Q15649 & DDDDSIADFLNSDEEEDR & Phospho(ST) \\
\hline Q8NEY1 & APEAAVSEDGKSDDELLSSK & Phospho(ST) \\
\hline P51532 & KIPDPDSDDVSEVDAR & Phospho(ST) \\
\hline P51532 & AKPVVSDDDSEEEQEEDR & 2 Phospho(ST) \\
\hline Q9BWW4 & NSPNNISGISNPPGTPR & Phospho(ST) \\
\hline Q96QT6 & TTSPSSDTDLLDR & Phospho(ST) \\
\hline O95155 & NNESQWKDSPLATR & Phospho(ST) \\
\hline O95155 & SQSSEGVSSLSSSPSNSLETQSQSLSR & Phospho(ST) \\
\hline Q13541 & NSPVTKTPPR & Phospho(ST) \\
\hline Q13541 & NSPVTKTPPR & 2 Phospho(ST) \\
\hline Q13541 & $\begin{array}{l}\text { VVLGDGVQLPPGDYSTTPGGTLFSTTPGGT } \\
\text { R }\end{array}$ & Phospho(ST) \\
\hline Q13541 & $\begin{array}{l}\text { VVLGDGVQLPPGDYSTTPGGTLFSTTPGGT } \\
\text { R }\end{array}$ & 2 Phospho(ST) \\
\hline Q13541 & $\begin{array}{l}\text { RVVLGDGVQLPPGDYSTTPGGTLFSTTPGG } \\
\text { TR }\end{array}$ & 2 Phospho(ST) \\
\hline Q9UPN7 & SGGSTDSEDEEEEDEEEEEDEEGIGCAAR & 3 Phospho(ST) \\
\hline Q14694 & NHSVNEEEQEEQGEGSEDEWEQVGPR & Phospho(ST) \\
\hline Q9UBQ5 & IDFDSVSSIMASSQ & Phospho(ST) \\
\hline Q8TDD1 & GSDSEDGEFEIQAEDDAR & 2 Phospho(ST) \\
\hline Q4G0J3 & DIEISTEEEKDTGDLK & 2 Phospho(ST) \\
\hline Q4G0J3 & SRPTSEGSDIESTEPQK & 2 Phospho(ST) \\
\hline P27708 & IHRASDPGLPAEEPK & Phospho(ST) \\
\hline P62995 & SKEDSRR & Phospho(ST) \\
\hline P62995 & SRSKEDSR & Phospho(ST) \\
\hline P62995 & RSPSPYYSR & 2 Phospho(ST) \\
\hline P62995 & RRSPSPYYSR & 2 Phospho(ST) \\
\hline P62995 & RRSPSPYYSR & Phospho(ST) \\
\hline Q13595 & SYTPEYR & Phospho(ST) \\
\hline Q13595 & RSPSPYYSR & 2 Phospho(ST) \\
\hline Q13595 & RRSPSPYYSR & 2 Phospho(ST) \\
\hline Q13595 & RRSPSPYYSR & Phospho(ST) \\
\hline O75909 & KPSPQPSSPR & Phospho(ST) \\
\hline Q9UIG0 & SLSGSPLK & Phospho(ST) \\
\hline Q9UIG0 & $\begin{array}{l}\text { SDVQEESEGSDTDDNKDSAAFEDNEVQDEF } \\
\text { LEK }\end{array}$ & 2 Phospho(ST) \\
\hline Q9UIG0 & $\begin{array}{l}\text { RSDVQEESEGSDTDDNKDSAAFEDNEVQD } \\
\text { EFLEK }\end{array}$ & 2 Phospho(ST) \\
\hline Q14980 & LGNSLLR & Phospho(ST) \\
\hline Q14980 & VSLEPHQGPGTPESK & Phospho(ST) \\
\hline Q14980 & APVPSTCSSTFPEELSPPSHQAK & Phospho(ST) \\
\hline Q9P258 & CSSSSGGGSSGDEDGLELDGAPGGGK & 2 Phospho(ST) \\
\hline Q9P258 & CSSSSGGGSSGDEDGLELDGAPGGGKR & 2 Phospho(ST) \\
\hline
\end{tabular}




\begin{tabular}{|c|c|c|}
\hline Protein & Peptide sequence & Variable modification \\
\hline Q9P258 & $\begin{array}{l}\text { ERPERCSSSSGGGSSGDEDGLELDGAPGGG } \\
\mathrm{K}\end{array}$ & 6 Phospho(ST) \\
\hline Q01082 & RPPSPEPSTK & Phospho(ST) \\
\hline Q01082 & ESSPIPSPTSDR & 2 Phospho(ST) \\
\hline Q96T60 & TPESQPDTPPGTPLVSQDEK & Phospho(ST) \\
\hline Q9BWG4 & SSPGAVAGLSNAPGTPR & Phospho(ST) \\
\hline P50552 & NSTTLPR & Phospho(ST) \\
\hline Q13200 & DKAPVQPQQSPAAAPGGTDEKPSGK & Phospho(ST) \\
\hline O75821 & VTNLSEDTR & Phospho(ST) \\
\hline O75821 & GIPLATGDTSPEPELLPGAPLPPPK & Phospho(ST) \\
\hline O75821 & GIPLATGDTSPEPELLPGAPLPPPK & 2 Phospho(ST) \\
\hline Q96C19 & ADLNQGIGEPQSPSR & Phospho(ST) \\
\hline Q9BQG0 & EIPSATQSPISK & Phospho(ST) \\
\hline Q9BQG0 & EIPSATQSPISK & 2 Phospho(ST) \\
\hline Q9BXP5 & HELSPPQK & Phospho(ST) \\
\hline Q9BXP5 & HELSPPQKR & Phospho(ST) \\
\hline Q9C086 & AWLDEDSNLSPSPLR & 2 Phospho(ST) \\
\hline Q13501 & LTPVSPESSSTEEK & Phospho(ST) \\
\hline Q13501 & $\begin{array}{l}\text { EVDPSTGELQSLQMPESEGPSSLDPSQEGPT } \\
\text { GLK }\end{array}$ & Phospho(ST) \\
\hline Q9Y608 & RGSGDTSSLIDPDTSLSELR & Phospho(ST) \\
\hline Q8ND76 & SASADNLTLPR & Phospho(ST) \\
\hline Q86UE4 & LSSQISAGEEK & Phospho(ST) \\
\hline Q9Y2X7 & HGSGADSDYENTQSGDPLLGLEGK & 2 Phospho(ST) \\
\hline Q9Y2X7 & SQSDLDDQHDYDSVASDEDTDQEPLR & 2 Phospho(ST) \\
\hline Q9BZ23 & LGSYSGPTSVSR & Phospho(ST) \\
\hline P12268 & LVGIISSR & Phospho(ST) \\
\hline Q96T23 & KPDSPPK & Phospho(ST) \\
\hline Q96T23 & DAQRLSPIPEEVPK & Phospho(ST) \\
\hline Q96T23 & STLESEKPGSPEAAETSPPSNIIDHCEK & Phospho(ST) \\
\hline O76021 & AAESETPGKSPEK & Phospho(ST) \\
\hline O76021 & KSPAKSPNPSTPR & 2 Phospho(ST) \\
\hline O76021 & ATNESEDEIPQLVPIGK & Phospho(ST) \\
\hline Q96125 & RPDPDSDEDEDYER & Phospho(ST) \\
\hline Q96BT3 & AASPESASSTPESLQAR & Phospho(ST) \\
\hline Q8TC07 & NDSPTQIPVSSDVCR & Phospho(ST) \\
\hline Q69YN4 & SFLSEPSSPGR & Phospho(ST) \\
\hline Q69YN4 & EDYFEPISPDR & Phospho(ST) \\
\hline Q14689 & IQQLLNTLK & Phospho(ST) \\
\hline P08648 & LLESSLSSSEGEEPVEYK & Phospho(ST) \\
\hline Q9C0I1 & QLSLPLTQSK & Phospho(ST) \\
\hline Q96HC4 & EVVKPVPITSPAVSK & Phospho(ST) \\
\hline Q5T4S7 & HVTLPSSPR & Phospho(ST) \\
\hline
\end{tabular}




\begin{tabular}{|c|c|c|}
\hline Protein & Peptide sequence & Variable modification \\
\hline Q5T4S7 & AAPPPPPPPPPLESSPR & Phospho(ST) \\
\hline Q05209 & NLSFEIK & Phospho(ST) \\
\hline Q05209 & DVDVSEDSPPPLPER & Phospho(ST) \\
\hline Q86W92 & SSSLGNLK & Phospho(ST) \\
\hline Q9H7L9 & GRESDEDTEDASETDLAK & 3 Phospho(ST) \\
\hline Q9H7L9 & RPASPSSPEHLPATPAESPAQR & Phospho(ST) \\
\hline Q9H7L9 & RPASPSSPEHLPATPAESPAQR & 2 Phospho(ST) \\
\hline P40818 & SYSSPDITQAIQEEEKR & Phospho(ST) \\
\hline Q147X3 & SPAGGESATVAAK & Phospho(ST) \\
\hline Q9BZC7 & VSEEDQSLENSEADVK & 2 Phospho(ST) \\
\hline O15014 & LVEPHSPSPSSK & 2 Phospho(ST) \\
\hline Q8IWS0 & TAHNSEADLEESFNEHELEPSSPK & Phospho(ST) \\
\hline Q7KZI7 & STFHAGQLR & Phospho(ST) \\
\hline Q7KZI7 & SRNSPLLER & Phospho(ST) \\
\hline Q7KZI7 & VPASPLPGLER & Phospho(ST) \\
\hline Q9NS91 & NDLQDTEISPR & Phospho(ST) \\
\hline Q92619 & AGSPSPQPSGELPR & Phospho(ST) \\
\hline Q8ND56 & SPVSTRPLPSASQK & Phospho(ST) \\
\hline P08559 & YHGHSMSDPGVSYR & Phospho(ST) \\
\hline P08559 & YHGHSMSDPGVSYR & 2 Phospho(ST) \\
\hline O95453 & NNSFTAPSTVGK & Phospho(ST) \\
\hline Q9NUQ6 & SQGSGNEAEPLGK & Phospho(ST) \\
\hline Q9UQ88 & DLLSDLQDISDSER & 2 Phospho(ST) \\
\hline Q9UQ88 & GTSPRPPEGGLGYSQLGDDDLK & Phospho(ST) \\
\hline P28290 & SLASIEAK & Phospho(ST) \\
\hline P28290 & SKTPLVAR & Phospho(ST) \\
\hline P28290 & TGVPSTASVGK & Phospho(ST) \\
\hline P28290 & SQSLPTTLLSPVR & 2 Phospho(ST) \\
\hline P28290 & TGSVQTPPDLESSEEVDAAEGAPEVVGPK & Phospho(ST) \\
\hline Q01130 & SKSPPKSPEEEGAVSS & 2 Phospho(ST) \\
\hline Q01130 & SKSPPKSPEEEGAVSS & 3 Phospho(ST) \\
\hline Q01130 & SKSPPKSPEEEGAVSS & 4 Phospho(ST) \\
\hline Q9NQ55 & VGGSDEEASGIPSR & Phospho(ST) \\
\hline Q9NQ55 & $\begin{array}{l}\text { LQDISELLATGAGLSESEAEPDGDHNITELP } \\
\text { QAVAGR }\end{array}$ & 2 Phospho(ST) \\
\hline Q08AD1 & ADVPVEKYDGESDKEQFDDDQK & Phospho(ST) \\
\hline Q96ER3 & NPSPPPPGR & Phospho(ST) \\
\hline Q96ER3 & SSSDDEEQLTELDEEMENEICR & Phospho(ST) \\
\hline P15408 & QEPLEEDSPSSSSAGLDK & Phospho(ST) \\
\hline Q9H2Y7 & SLSESSVIMDR & Phospho(ST) \\
\hline Q9H2Y7 & EPHSPADQPEQQAESTLTSAETR & Phospho(ST) \\
\hline O75400 & SDSPESDAER & Phospho(ST) \\
\hline Q86XP3 & QQFHSKPVDSDSDDDPLEAFMAEVEDQAA & 2 Phospho(ST) \\
\hline
\end{tabular}




\begin{tabular}{|c|c|c|}
\hline Protein & Peptide sequence & Variable modification \\
\hline & $\mathrm{R}$ & \\
\hline Q9BTU6 & VAAAAGSGPSPPGSPGHDR & Phospho(ST) \\
\hline Q9BTU6 & VAAAAGSGPSPPGSPGHDR & 2 Phospho(ST) \\
\hline O94875 & RKSEPAVGPPR & Phospho(ST) \\
\hline O94875 & DASSPVPPPHVPPPVPPLRPR & Phospho(ST) \\
\hline Q96MU7 & GISPIVFDR & Phospho(ST) \\
\hline Q7L4I2 & EQSEVSVSPR & Phospho(ST) \\
\hline Q7L4I2 & KEQSEVSVSPR & Phospho(ST) \\
\hline Q7L4I2 & DGLAPEKTSPDRDK & Phospho(ST) \\
\hline P45880 & LTFDTTFSPNTGK & Phospho(ST) \\
\hline Q14157 & KASLTSK & Phospho(ST) \\
\hline Q14157 & RYPSSISSSPQK & Phospho(ST) \\
\hline Q01804 & VQRPKEESSEDENEVSNILR & 2 Phospho(ST) \\
\hline Q9H4G0 & SLSPIIGK & Phospho(ST) \\
\hline Q9H4G0 & RLPSSPASPSPK & Phospho(ST) \\
\hline Q9H4G0 & SLDGAEFSRPASVSENHDAGPDGDKR & 2 Phospho(ST) \\
\hline Q9H792 & ANTLSPVR & Phospho(ST) \\
\hline Q9H792 & SHSSPSQIPK & Phospho(ST) \\
\hline Q9P2B4 & VSSPLSPLSPGIKSPTIPR & Phospho(ST) \\
\hline Q9P2B4 & FQSQADQDQQASGLQSPPSR & Phospho(ST) \\
\hline Q9UKS6 & DLHQGIEAASDEEDLR & Phospho(ST) \\
\hline Q9UKS6 & DGTAPPPQSPGSPGTGQDEEWSDEESPR & Phospho(ST) \\
\hline Q9UKS6 & DGTAPPPQSPGSPGTGQDEEWSDEESPRK & 2 Phospho(ST) \\
\hline O75925 & GILSLPHQASPVSR & Phospho(ST) \\
\hline O75925 & KVEVIDLTIDSSSDEEEEEPSAK & 3 Phospho(ST) \\
\hline Q9H307 & EIAIVHSDAEK & Phospho(ST) \\
\hline Q9H307 & QESDPEDDDVKKPALQSSVVATSK & Phospho(ST) \\
\hline Q9P289 & LADFGVAGQLTDTQIK & Phospho(ST) \\
\hline Q9UQN3 & ATISDEEIER & Phospho(ST) \\
\hline Q9H8Y5 & EALGQNEESPK & Phospho(ST) \\
\hline O95400 & KGPGQPSSPQR & Phospho(ST) \\
\hline P24928 & YSPTSPTYSPTSPK & Phospho(ST) \\
\hline P24928 & YSPTSPTYSPTSPVYTPTSPK & Phospho(ST) \\
\hline Q6ZW31 & DFLSGPDYDHVTGSDSEDEDEEVGEPR & 2 Phospho(ST) \\
\hline Q15361 & YLSADSGDADDSDADLGSAVK & Phospho(ST) \\
\hline P09651 & SESPKEPEQLR & Phospho(ST) \\
\hline Q9H3Q1 & AGPDLPSLPSHALEDEGWAAAAPSPGSAR & Phospho(ST) \\
\hline Q9H3Q1 & AGPDLPSLPSHALEDEGWAAAAPSPGSAR & 2 Phospho(ST) \\
\hline Q6UN15 & AEFTSPPSLFK & Phospho(ST) \\
\hline Q6UN15 & DHSPTPSVFNSDEER & 2 Phospho(ST) \\
\hline Q6UN15 & VTETEDDSDSDSDDDEDDVHVTIGDIK & 3 Phospho(ST) \\
\hline P17544 & TDSVIIADQTPTPTR & Phospho(ST) \\
\hline Q15059 & SESPPPLSDPK & Phospho(ST) \\
\hline
\end{tabular}




\begin{tabular}{|c|c|c|}
\hline Protein & Peptide sequence & Variable modification \\
\hline Q8IWA0 & VQDTSNTGLGEDIIHQLSK & Phospho(ST) \\
\hline Q86V48 & EKPDSDDDLDIASLVTAK & Phospho(ST) \\
\hline P82094 & SATPVNCEQPDILVSSTPINEGQTVLDK & Phospho(ST) \\
\hline Q9HCK8 & VSPSDTTPLVSR & Phospho(ST) \\
\hline Q9HCK8 & TASPLPLRPDAPVEK & Phospho(ST) \\
\hline Q9HCK8 & APGYPSSPVTTASGTTLR & Phospho(ST) \\
\hline Q9HCK8 & DDDLVEFSDLESEDDERPR & 2 Phospho(ST) \\
\hline Q9HCK8 & $\begin{array}{l}\text { TASPLPLRPDAPVEKSPEETATQVPSLESLT } \\
\text { LK }\end{array}$ & 2 Phospho(ST) \\
\hline P51114 & RGPNYTSGYGTNSELSNPSETESER & Phospho(ST) \\
\hline P0DJ93 & ELVGDTGSQEGDHEPSGSETEEDTSSSPHR & 2 Phospho(ST) \\
\hline P98082 & SSPNPFVGSPPK & Phospho(ST) \\
\hline P98082 & SSPNPFVGSPPK & 2 Phospho(ST) \\
\hline P16383 & AADSSDSDGAEESPAEPGAPR & 3 Phospho(ST) \\
\hline Q7RTP6 & GPSQATSPIR & Phospho(ST) \\
\hline Q5BKZ1 & ISLSKSPTK & Phospho(ST) \\
\hline O15234 & STVTGERQSGDGQESTEPVENK & Phospho(ST) \\
\hline P78347 & ESTSSKSPPR & Phospho(ST) \\
\hline P78347 & SILSPGGSCGPIK & Phospho(ST) \\
\hline P78347 & TNTPVKEDWNVR & Phospho(ST) \\
\hline P42684 & VPVLISPTLK & Phospho(ST) \\
\hline Q9NRG9 & FSPVLGR & Phospho(ST) \\
\hline Q9BX95 & RNSLTGEEGQLAR & Phospho(ST) \\
\hline Q04637 & KAASLTEDR & Phospho(ST) \\
\hline Q04637 & SFSKEVEER & Phospho(ST) \\
\hline Q96B97 & SIEVENDFLPVEK & Phospho(ST) \\
\hline Q9NY61 & YLVDGTKPNAGSEEISSEDDELVEEK & 2 Phospho(ST) \\
\hline P46937 & QASTDAGTAGALTPQHVR & Phospho(ST) \\
\hline Q9Y2U8 & VLLGFSSDESDVEASPR & 3 Phospho(ST) \\
\hline Q9Y2U8 & ENYSDSEEEDDDDVASSR & Phospho(ST) \\
\hline Q5T1M5 & SSLSGDEEDELFK & 2 Phospho(ST) \\
\hline Q8IWZ3 & LNLTSPK & Phospho(ST) \\
\hline Q8IWZ3 & TVSLPLSSPNIK & Phospho(ST) \\
\hline Q9H6X2 & EVPPPPAEESEEEDDDGLPK & Phospho(ST) \\
\hline Q92769 & IACDEEFSDSEDEGEGGR & 2 Phospho(ST) \\
\hline Q92769 & RIACDEEFSDSEDEGEGGR & 2 Phospho(ST) \\
\hline O00512 & AVTPVSQGSNSSSADPK & Phospho(ST) \\
\hline Q8N8A6 & RVNDAEPGSPEAPQGK & Phospho(ST) \\
\hline Q6ZRS2 & AGAPVGGSPGLAK & Phospho(ST) \\
\hline Q99961 & IAASSSFR & Phospho(ST) \\
\hline O95831 & SLSAIDR & Phospho(ST) \\
\hline P98175 & LASDDRPSPPR & Phospho(ST) \\
\hline Q86UU1 & ALSPLPTR & Phospho(ST) \\
\hline
\end{tabular}




\begin{tabular}{|c|c|c|}
\hline Protein & Peptide sequence & Variable modification \\
\hline Q86UU1 & TFSDGLATR & Phospho(ST) \\
\hline Q86UU1 & ELPPLSPSLSR & Phospho(ST) \\
\hline Q86UU1 & KNSITEISDNEDDLLEYHR & 2 Phospho(ST) \\
\hline P49790 & NTSLPPLWSPEAER & Phospho(ST) \\
\hline Q6WCQ1 & SKSNPDFLKK & Phospho(ST) \\
\hline Q08499 & DSGSQVEEDTSCSDSK & Phospho(ST) \\
\hline Q08499 & EWYQSTIPQSPSPAPDDPEEGR & Phospho(ST) \\
\hline Q9Y5S2 & HSTPSNSSNPSGPPSPNSPHR & Phospho(ST) \\
\hline Q8IVF2 & IKLPSFR & Phospho(ST) \\
\hline Q8IVF2 & LGFSSSPTK & Phospho(ST) \\
\hline Q8IVF2 & IPSLGWSPSK & Phospho(ST) \\
\hline Q9NYV4 & GSPVFLPR & Phospho(ST) \\
\hline Q9NYV4 & HLLTDLPLPPELPGGDLSPPDSPEPK & 2 Phospho(ST) \\
\hline Q9BUQ8 & KSSPSKDR & Phospho(ST) \\
\hline Q9P2D1 & NIPSPGQLDPDTR & Phospho(ST) \\
\hline Q9BUA3 & AESPSPAPPPGLR & Phospho(ST) \\
\hline Q9NUQ3 & NLVSPAYCTQESR & Phospho(ST) \\
\hline Q14004 & SLSPLGGR & 2 Phospho(ST) \\
\hline Q14004 & IEHAPSPSSGGTLK & Phospho(ST) \\
\hline Q14004 & ILELTPEPDRPR & Phospho(ST) \\
\hline Q14004 & TNTPQGVLPSSQLK & Phospho(ST) \\
\hline O43491 & GSQPPPAAESQSSLRR & Phospho(ST) \\
\hline Q9BRQ0 & GGGTPDANSLAPPGK & Phospho(ST) \\
\hline O15318 & GDGEKSDEENEEK & Phospho(ST) \\
\hline P48681 & QEASTGQSPEDHASLAPPLSPDHSSLEAK & Phospho(ST) \\
\hline Q9NSK0 & VSSPTVNTTLR & Phospho(ST) \\
\hline Q9ULM3 & VQSPKPITGGLGAFTK & Phospho(ST) \\
\hline Q8N684 & SSSTEPPPPVR & Phospho(ST) \\
\hline Q8N684 & DSSDSADGRATPSENLVPSSAR & Phospho(ST) \\
\hline O66678 & $\begin{array}{l}\text { GAVENEEDLPELSDSGDEAAWEDEDDADL } \\
\text { PHGK }\end{array}$ & 2 Phospho(ST) \\
\hline Q9UHR5 & GGLVSDAYGEDDFSR & Phospho(ST) \\
\hline P35658 & ITPPAAKPGSPQAK & Phospho(ST) \\
\hline P35658 & SPGSTPTTPTSSQAPQK & 2 Phospho(ST) \\
\hline Q9ULU4 & EKETSAEKSK & Phospho(ST) \\
\hline Q9ULU4 & TGQAGSLSGSPKPFSPQLSAPITTK & Phospho(ST) \\
\hline P55010 & EAEEESSGGEEEDEDENIEVVYSK & 2 Phospho(ST) \\
\hline Q9NZ63 & VGDTEKPEPERSPPNR & Phospho(ST) \\
\hline Q9NZ63 & RRGDSESEEDEQDSEEVR & 2 Phospho(ST) \\
\hline Q96FS4 & SGSDAGEARPPTPASPR & Phospho(ST) \\
\hline Q15942 & GPPASSPAPAPK & Phospho(ST) \\
\hline Q8NEY8 & KDSPHSR & Phospho(ST) \\
\hline Q8NEY8 & DNTFFRESPVGR & Phospho(ST) \\
\hline
\end{tabular}




\begin{tabular}{|c|c|c|}
\hline Protein & Peptide sequence & Variable modification \\
\hline Q5VTL8 & SQSIEQESQEK & 2 Phospho(ST) \\
\hline Q5VTL8 & RRSQSIEQESQEK & 2 Phospho(ST) \\
\hline Q9Y463 & IYQYIQSR & Phospho(Y) \\
\hline Q12929 & QNSSSSDSGGSIVR & 2 Phospho(ST) \\
\hline Q8TDM6 & VGPCSSPPAAR & Phospho(ST) \\
\hline Q9Y613 & SLEGGGCPAR & Phospho(ST) \\
\hline P54727 & QEKPAEKPAETPVATSPTATDSTSGDSSR & Phospho(ST) \\
\hline Q14155 & EDLSKSPK & Phospho(ST) \\
\hline Q9BRJ6 & ELDEEGSDPPLPGR & Phospho(ST) \\
\hline Q13111 & ATLTASPLGAS & Phospho(ST) \\
\hline Q13111 & SCPELTSGPR & Phospho(ST) \\
\hline Q5THJ4 & NSSSESAIVPK & Phospho(ST) \\
\hline Q9P2N5 & LQLGTPPPLLAAR & Phospho(ST) \\
\hline O60292 & TLSDESLCSGR & Phospho(ST) \\
\hline Q9BSJ6 & GSGSPTHSLSQK & Phospho(ST) \\
\hline O00443 & ETLRQR & Phospho(ST) \\
\hline O00443 & SQSLNIR & Phospho(ST) \\
\hline Q8TF01 & SNRNSIER & Phospho(ST) \\
\hline Q9UNN5 & TPSGEFLER & Phospho(ST) \\
\hline Q9BUJ2 & APQQQPPPQQPPPPQPPPQQPPPPPSYSPAR & Phospho(ST) \\
\hline Q96G23 & LVEDERSDR & Phospho(ST) \\
\hline Q96G23 & LVEDERSDREETESSEGEEAAAGGGAK & 4 Phospho(ST) \\
\hline P16989 & AGEAPTENPAPPTQQSSAE & Phospho(ST) \\
\hline P16989 & NYAGEEEEEGSGSSEGFDPPATDR & Phospho(ST) \\
\hline P16989 & $\begin{array}{l}\text { SPVGSGAPQAAAPAPAAHVAGNPGGDAAP } \\
\text { AATGTAAAASLATAAGSEDAEK }\end{array}$ & Phospho(ST) \\
\hline Q9UDT6 & IGFPSTSPAK & Phospho(ST) \\
\hline Q9H019 & NASVPNLR & Phospho(ST) \\
\hline Q13136 & RSSDGSLSHEEDLAK & 2 Phospho(ST) \\
\hline Q5SZK8 & SRAGIAISAFNLK & Phospho(ST) \\
\hline P48634 & ERSDSGGSSSEPFDR & 2 Phospho(ST) \\
\hline P48634 & GSETGSETHESDLAPSDK & Phospho(ST) \\
\hline P48634 & TASETRSEGSEYEEIPK & 2 Phospho(ST) \\
\hline P48634 & LKFSDEEDGRDSDEEGAEGHR & 2 Phospho(ST) \\
\hline P0DP91 & $\begin{array}{l}\text { KVPVQEIDDDFFPSSGEEAEAASVGEGGGG } \\
\text { GR }\end{array}$ & 2 Phospho(ST) \\
\hline Q6R327 & STELLLGVK & Phospho(ST) \\
\hline Q7Z6Z7 & DGGSGNSTIIVSR & Phospho(ST) \\
\hline O75494 & SFDYNYR & Phospho(ST) \\
\hline P14859 & INPPSSGGTSSSPIK & Phospho(ST) \\
\hline P08621 & YDERPGPSPLPHR & Phospho(ST) \\
\hline P12694 & SVDEVNYWDK & Phospho(ST) \\
\hline P53999 & ELVSSSSSGSDSDSEVDKK & Phospho(ST) \\
\hline
\end{tabular}




\begin{tabular}{|c|c|c|}
\hline Protein & Peptide sequence & Variable modification \\
\hline P17275 & DATPPVSPINMEDQER & 2 Phospho(ST) \\
\hline P17275 & SRDATPPVSPINMEDQER & 3 Phospho(ST) \\
\hline P47914 & RTQAPTKASE & Phospho(ST) \\
\hline O60885 & IHSPIIR & Phospho(ST) \\
\hline O00629 & NVPHEDICEDSDIDGDYR & Phospho(ST) \\
\hline $\mathrm{O} 15042$ & RVKSPSPK & 2 Phospho(ST) \\
\hline $\mathrm{O} 15042$ & LYSILQGDSPTK & Phospho(ST) \\
\hline Q3YEC7 & RSIISR & Phospho(ST) \\
\hline Q3YEC7 & DDPSDVTDEDEGPAEPPPPPK & 2 Phospho(ST) \\
\hline Q3YEC7 & DDPSDVTDEDEGPAEPPPPPKLPLPAFR & 2 Phospho(ST) \\
\hline Q3YEC7 & $\begin{array}{l}\text { AAQQDSDSDGEALGGNPMVAGFQDDVDL } \\
\text { EDQPR }\end{array}$ & 2 Phospho(ST) \\
\hline Q07955 & VDGPRSPSYGR & Phospho(ST) \\
\hline Q07955 & VDGPRSPSYGR & 2 Phospho(ST) \\
\hline Q07955 & VKVDGPRSPSYGR & 2 Phospho(ST) \\
\hline Q9ULT0 & LPNSIASR & Phospho(ST) \\
\hline Q8WYP5 & EVSPSDVR & Phospho(ST) \\
\hline Q9BYX2 & AVSEGCASEDEVEGEA & Phospho(ST) \\
\hline Q2M2I8 & VGSLTPPSSPK & 2 Phospho(ST) \\
\hline Q9BY89 & TSSPLFNK & Phospho(ST) \\
\hline Q5VZ89 & STSLSALVR & Phospho(ST) \\
\hline Q15036 & VTSSVPLPSGSTSSPGR & Phospho(ST) \\
\hline Q96F63 & LLQQQEEEEACLEEEEEEEDSDEEDQR & Phospho(ST) \\
\hline Q15758 & GPAGDATVASEK & Phospho(ST) \\
\hline Q15758 & STEPELIQVK & Phospho(ST) \\
\hline Q6P4Q7 & SASLSYPDR & Phospho(ST) \\
\hline Q8NHG8 & DRPVGGSPGGPR & Phospho(ST) \\
\hline Q00839 & $\begin{array}{l}\text { AKSPQPPVEEEDEHFDDTVVCLDTYNCDLH } \\
\text { FK }\end{array}$ & Phospho(ST) \\
\hline P54725 & EDKSPSEESAPTTSPESVSGSVPSSGSSGR & Phospho(ST) \\
\hline Q9ULX6 & $\begin{array}{l}\text { ATRTDCSDNSDSDNDEGTEGEATEGLEGTE } \\
\text { AVEK }\end{array}$ & 4 Phospho(ST) \\
\hline Q9ULX6 & $\begin{array}{l}\text { ATRTDCSDNSDSDNDEGTEGEATEGLEGTE } \\
\text { AVEK }\end{array}$ & 5 Phospho(ST) \\
\hline Q9UH99 & RGTGGSESSRASGLVGR & 4 Phospho(ST) \\
\hline Q5SXM2 & VGSESEDEDLLSELELADR & 2 Phospho(ST) \\
\hline Q5SXM2 & VGSESEDEDLLSELELADR & 3 Phospho(ST) \\
\hline Q92945 & VQISPDSGGLPER & Phospho(ST) \\
\hline Q5T5U3 & AQPSSSEDELDNVFFK & 2 Phospho(ST) \\
\hline Q5T5U3 & SELISEGRPVETDSESEFPVFPTALTSER & 2 Phospho(ST) \\
\hline O76094 & TVSSPPTSPRPGSAATVSASTSNIIPPR & 2 Phospho(ST) \\
\hline Q9UJX2 & RVSPLNLSSVTP & Phospho(ST) \\
\hline P29375 & DLDLEPLSDLEEGLEETR & Phospho(ST) \\
\hline
\end{tabular}




\begin{tabular}{|c|c|c|}
\hline Protein & Peptide sequence & Variable modification \\
\hline Q92466 & SRSPLELEPEAK & 2 Phospho(ST) \\
\hline P18887 & TKPTQAAGPSSPQKPPTPEETK & Phospho(ST) \\
\hline Q15311 & TEGYAAFQEDSSGDEAESPSK & 2 Phospho(ST) \\
\hline Q7Z5K2 & VEEESTGDPFGFDSDDESLPVSSK & Phospho(ST) \\
\hline Q7Z5K2 & YFGFDDLSESEDDEDDDCQVER & 2 Phospho(ST) \\
\hline O75376 & EPAPLLSAQYETLSDSDD & 2 Phospho(ST) \\
\hline O75376 & HEAPSSPISGQPCGDDQNASPSK & 2 Phospho(ST) \\
\hline Q9NPF5 & DTIIDVVGAPLTPNSR & Phospho(ST) \\
\hline Q9NQW6 & GNIWSAEK & Phospho(ST) \\
\hline Q9NQW6 & TQSLPVTEK & Phospho(ST) \\
\hline O00273 & SISASKASPPGDLQNPK & Phospho(ST) \\
\hline Q00613 & VKEEPPSPPQSPR & 2 Phospho(ST) \\
\hline Q00613 & GHTDTEGRPPSPPPTSTPEK & Phospho(ST) \\
\hline Q86TB9 & STSPIIGSPPVR & Phospho(ST) \\
\hline Q58WW2 & DSALQDTDDSDDDPVLIPGAR & 2 Phospho(ST) \\
\hline P07814 & EYIPGQPPLSQSSDSSPTR & Phospho(ST) \\
\hline O15164 & SEWLDPSQKSPLHVGETR & Phospho(ST) \\
\hline Q7Z569 & VLKETCDQK & Phospho(ST) \\
\hline Q7Z569 & ECINAAPDSPSK & Phospho(ST) \\
\hline Q66K74 & LSLSPLR & Phospho(ST) \\
\hline Q66K74 & LSLSPLR & 2 Phospho(ST) \\
\hline Q66K74 & AVPMAPAPASPGSSNDSSAR & Phospho(ST) \\
\hline Q13017 & GGIDNPAITSDQELDDKK & Phospho(ST) \\
\hline Q8WX92 & KPSPAQAAETPALELPLPSVPAPAPL & Phospho(ST) \\
\hline O60524 & DELNEELIQEESSEDEGEYEEVRK & 2 Phospho(ST) \\
\hline Q86X95 & NLTANDPSQEYVASEGEEDPEVEFLK & Phospho(ST) \\
\hline Q14680 & VFGSLER & Phospho(ST) \\
\hline Q9Y5J1 & VQEHEDSGDSEVENEAK & 2 Phospho(ST) \\
\hline Q9Y5J1 & KTSSDDESEEDEDDLLQR & 2 Phospho(ST) \\
\hline E9PAV3 & $\begin{array}{l}\text { VQGEAVSNIQENTQTPTVQEESEEEEVDET } \\
\text { GVEVK }\end{array}$ & Phospho(ST) \\
\hline Q9BW61 & TDSPDMHEDT & Phospho(ST) \\
\hline P00519 & AGGKPSQSPSQEAAGEAVLGAK & Phospho(ST) \\
\hline P00519 & GQGESDPLDHEPAVSPLLPR & Phospho(ST) \\
\hline O75448 & LLSSNEDDANILSSPTDR & Phospho(ST) \\
\hline Q15424 & SVVSFDK & Phospho(ST) \\
\hline Q15424 & SVVSFDKVK & 2 Phospho(ST) \\
\hline Q15424 & SVVSFDKVKEPR & Phospho(ST) \\
\hline Q15424 & ETINNLDTSSSDFTILQEIEEPSLEPENEK & Phospho(ST) \\
\hline P26641 & KDTPRK & Phospho(ST) \\
\hline P26641 & TPEFLR & Phospho(ST) \\
\hline Q07960 & SSSPELVTHLK & Phospho(ST) \\
\hline Q8IZ73 & VELSPGPPKPAGR & Phospho(ST) \\
\hline
\end{tabular}




\begin{tabular}{|c|c|c|}
\hline Protein & Peptide sequence & Variable modification \\
\hline Q7Z422 & KSKSPPK & Phospho(ST) \\
\hline Q7Z422 & SKSPPKVPIVIQDDSLPAGPPPQIR & Phospho(ST) \\
\hline Q9H6Z4 & SAGGSSPEGGEDSDREDGNYCPPVK & 2 Phospho(ST) \\
\hline Q86Y82 & AGSRLSAEER & Phospho(ST) \\
\hline Q96B23 & RDSSESQLASTESDKPTTGR & 2 Phospho(ST) \\
\hline Q86SQ0 & SGAASMPSSPK & Phospho(ST) \\
\hline Q9NZH5 & LFQLGPPSPVK & Phospho(ST) \\
\hline Q5T0N5 & TISDGTISASK & Phospho(ST) \\
\hline Q9ULW0 & SSDQPLTVPVSPK & Phospho(ST) \\
\hline P30622 & TASESISNLSEAGSIK & 3 Phospho(ST) \\
\hline A0FGR8 & EPTPSIASDISLPIATQELR & 2 Phospho(ST) \\
\hline A0FGR8 & EPTPSIASDISLPIATQELR & 3 Phospho(ST) \\
\hline Q8NFG4 & AHSPAEGASVESSSPGPK & 2 Phospho(ST) \\
\hline Q01167 & EGSPAPLEPEPGAAQPK & Phospho(ST) \\
\hline Q12797 & SSGNSSSSGSGSGSTSAGSSSPGAR & Phospho(ST) \\
\hline P53985 & EEETSIDVAGKPNEVTK & Phospho(ST) \\
\hline P53985 & KESKEEETSIDVAGKPNEVTK & Phospho(ST) \\
\hline Q9UJX6 & $\begin{array}{l}\text { TDPASLETGQDSEDDSGEPEDWVPDPVDA } \\
\text { DPGK }\end{array}$ & Phospho(ST) \\
\hline P16615 & EFDELNPSAQR & Phospho(ST) \\
\hline P26599 & TDSSPNQAR & Phospho(ST) \\
\hline Q5TDH0 & QPPGTQQSHSSPGEITSSPQGLDNPALLR & Phospho(ST) \\
\hline Q13769 & ALFKPPEDSQDDESDSDAEEEQTTK & 2 Phospho(ST) \\
\hline O75592 & SKSDSYTLDPDTLR & Phospho(ST) \\
\hline P49768 & AAVQELSSSILAGEDPEER & Phospho(ST) \\
\hline Q8WUM0 & RSSLSSR & Phospho(ST) \\
\hline Q96T17 & RPSSPVISK & Phospho(ST) \\
\hline Q8NFH5 & SVMESSDR & Phospho(ST) \\
\hline Q8NFH5 & SPLLAGGSPPQPVVPAHK & Phospho(ST) \\
\hline P51116 & TDGSISGDRQPVTVADYISR & 2 Phospho(ST) \\
\hline Q52LA3 & SPITSSPPK & Phospho(ST) \\
\hline Q8NFJ5 & AHAWPSPYK & Phospho(ST) \\
\hline Q567U6 & QSELSAEESPEK & Phospho(ST) \\
\hline P28066 & GVNTFSPEGR & Phospho(ST) \\
\hline P17535 & $\begin{array}{l}\text { LAALKDEPQTVPDVPSFGESPPLSPIDMDTQ } \\
\text { ER }\end{array}$ & 2 Phospho(ST) \\
\hline O95835 & QITTSPITVR & Phospho(ST) \\
\hline O94806 & SVLPTAIPAVLPAASPCSSPK & 2 Phospho(ST) \\
\hline P78563 & NLDNVSPK & Phospho(ST) \\
\hline Q15061 & LQAKESPQR & Phospho(ST) \\
\hline Q99504 & LSSGDPSTSPSLSQTTPSK & Phospho(ST) \\
\hline Q9BQA1 & KETPPPLVPPAAR & Phospho(ST) \\
\hline Q15418 & KLPSTTL & Phospho(ST) \\
\hline
\end{tabular}




\begin{tabular}{|c|c|c|}
\hline Protein & Peptide sequence & Variable modification \\
\hline Q3V6T2 & TGSPGSEVVTLQQFLEESNK & Phospho(ST) \\
\hline Q9P227 & SAEALGPGALVSPR & Phospho(ST) \\
\hline Q93100 & QTLCSLLPR & Phospho(ST) \\
\hline Q93100 & RQSSTPSAPELGQQPDVNISEWK & Phospho(ST) \\
\hline Q12874 & TGEEREEEEEEQISESESEDEENEIIYNPK & 3 Phospho(ST) \\
\hline Q14498 & YRSPYSGPK & Phospho(ST) \\
\hline Q14498 & DKSPVREPIDNLTPEER & Phospho(ST) \\
\hline Q5VV41 & GLNTSQESDDDILDESSSPEGTQK & Phospho(ST) \\
\hline Q8NC56 & WTKPSSFSDSER & Phospho(ST) \\
\hline O43583 & LTVENSPKQEAGISEGQGTAGEEEEK & Phospho(ST) \\
\hline Q5H9L2 & GTDDSPKDSQEDLQER & Phospho(ST) \\
\hline Q13427 & DQSPFSK & Phospho(ST) \\
\hline Q13427 & IRSSVEK & Phospho(ST) \\
\hline Q13427 & KSPPKADEK & Phospho(ST) \\
\hline Q13427 & NVSESPNRK & Phospho(ST) \\
\hline Q8NI27 & IDTHPSPSHSSTVK & Phospho(ST) \\
\hline Q7Z3C6 & HPEPVPEEGSEDELPPQVHKV & Phospho(ST) \\
\hline P39880 & AYQQKPYPSPK & Phospho(ST) \\
\hline O43290 & RVSEVEEEKEPVPQPLPSDDTR & Phospho(ST) \\
\hline P42858 & SGSIVELIAGGGSSCSPVLSR & Phospho(ST) \\
\hline P42858 & SGSIVELIAGGGSSCSPVLSR & 2 Phospho(ST) \\
\hline P49761 & YRSPEPDPYLSYR & Phospho(ST) \\
\hline O75781 & APAPSPAKEER & Phospho(ST) \\
\hline Q494U1 & EGSPEPWLPLTDGR & Phospho(ST) \\
\hline Q05655 & ASTFCGTPDYIAPEILQGLK & Phospho(ST) \\
\hline Q01814 & SATSSSPGSPIHSLETSL & Phospho(ST) \\
\hline O75794 & $\begin{array}{l}\text { DDPPTHSQPDSDDEAEEIQWSDDENTATLT } \\
\text { APEFPEFATK }\end{array}$ & 2 Phospho(ST) \\
\hline Q9Y5B0 & ENSPAAFPDR & Phospho(ST) \\
\hline Q86TC9 & TPVDESDDEIQHDEIPTGK & Phospho(ST) \\
\hline O60333 & SGLSLEELR & Phospho(ST) \\
\hline P19021 & GSGGLNLGNFFASR & Phospho(ST) \\
\hline Q99741 & LVLNTAVPDRLPAR & Phospho(ST) \\
\hline Q99741 & LEPTNVQTVTCSPR & Phospho(ST) \\
\hline Q16204 & SNSPDKFK & Phospho(ST) \\
\hline Q16204 & LDQPVSAPPSPR & 2 Phospho(ST) \\
\hline $\mathrm{O} 75533$ & WDQTADQTPGATPK & 2 Phospho(ST) \\
\hline $\mathrm{O} 75533$ & LSSWDQAETPGHTPSLR & 2 Phospho(ST) \\
\hline O75533 & KLSSWDQAETPGHTPSLR & 2 Phospho(ST) \\
\hline P25440 & KADTTTPTPTAILAPGSPASPPGSLEPK & 2 Phospho(ST) \\
\hline O75152 & $\begin{array}{l}\text { VESSENVPSPTHPPVVINAADDDEDDDDQF } \\
\text { SEEGDETK }\end{array}$ & Phospho(ST) \\
\hline $\mathrm{O} 75674$ & EATNTTSEPSAPSQDLLDLSPSPR & Phospho(ST) \\
\hline
\end{tabular}




\begin{tabular}{|c|c|c|}
\hline Protein & Peptide sequence & Variable modification \\
\hline Q9H6H4 & SFSMQDLR & Phospho(ST) \\
\hline Q9H6H4 & AGGLQDSDTEDECWSDTEAVPR & 3 Phospho(ST) \\
\hline Q13596 & $\begin{array}{l}\text { LPPPFPGLEPESEGAAGGSEPEAGDSDTEGE } \\
\text { DIFTGAAVVSK }\end{array}$ & 3 Phospho(ST) \\
\hline O14545 & LDSQPQETSPELPR & Phospho(ST) \\
\hline P85037 & SGGLQTPECLSR & Phospho(ST) \\
\hline P85037 & SGGLQTPECLSR & 2 Phospho(ST) \\
\hline P85037 & EGSPIPHDPEFGSK & Phospho(ST) \\
\hline P85037 & EEAPASPLRPLYPQISPLK & 2 Phospho(ST) \\
\hline Q9HC35 & RPSPAEK & Phospho(ST) \\
\hline Q09472 & TEIKEEEDQPSTSATQSSPAPGQSK & Phospho(ST) \\
\hline P0C1Z6 & ETLSPPR & Phospho(ST) \\
\hline P0C1Z6 & LLPYPTLASPASD & Phospho(ST) \\
\hline O60934 & IPNYQLSPTKLPSINK & Phospho(ST) \\
\hline Q9P260 & ENSPNSFPR & Phospho(ST) \\
\hline Q9HB19 & SQSYIPTSGCR & Phospho(ST) \\
\hline Q8N7B1 & KVSEPVK & Phospho(ST) \\
\hline Q9NWB6 & ASSPPDRIDIFGR & Phospho(ST) \\
\hline Q9H0B6 & RASSLNFLNK & Phospho(ST) \\
\hline Q9BY44 & SDKSPDLAPTPAPQSTPR & Phospho(ST) \\
\hline Q8IWW6 & ATTPPNQGRPDSPVYANLQELK & 2 Phospho(ST) \\
\hline Q5JSZ5 & EAGSPAQEFK & Phospho(ST) \\
\hline Q5JSZ5 & LKFSDDEEEEEVVK & Phospho(ST) \\
\hline Q07157 & DDISEIQSLASDHSGR & 2 Phospho(ST) \\
\hline Q07157 & VQIPVSRPDPEPVSDNEEDSYDEEIHDPR & 2 Phospho(ST) \\
\hline P24394 & SSPPTTPLR & Phospho(ST) \\
\hline Q96AY2 & QLSPEDSSSPVK & Phospho(ST) \\
\hline Q8WUI4 & KESAPPSLR & Phospho(ST) \\
\hline Q96JG6 & SPSVSPSKQPVSTSSK & 2 Phospho(ST) \\
\hline Q9NQS7 & VLAPILPDNFSTPTGSR & Phospho(ST) \\
\hline Q5SSJ5 & TVNSTRETPPK & Phospho(ST) \\
\hline O00571 & FSGGFGAR & Phospho(ST) \\
\hline P09874 & GGSDDSSKDPIDVNYEK & Phospho(ST) \\
\hline Q8WWY3 & SSGTASSVAFTPLQGLEIVNPQAAEK & 2 Phospho(ST) \\
\hline Q8WWY3 & SSGTASSVAFTPLQGLEIVNPQAAEKK & 2 Phospho(ST) \\
\hline Q96G46 & QENCGAQQVPAGPGTSTPPSSPVR & 2 Phospho(ST) \\
\hline Q86TN4 & KPLSLAGDEETECQSSPK & Phospho(ST) \\
\hline Q8N201 & LSSTPPLSALGR & Phospho(ST) \\
\hline Q9C0D5 & GVSMSLPSSPLLPR & 2 Phospho(ST) \\
\hline Q53GS9 & EVDEDSEPER & Phospho(ST) \\
\hline Q15287 & RFSPPR & Phospho(ST) \\
\hline Q13185 & RKSLSDSESDDSK & 2 Phospho(ST) \\
\hline Q8N9T8 & TASSSDSEEDPEALEK & 4 Phospho(ST) \\
\hline
\end{tabular}




\begin{tabular}{|c|c|c|}
\hline Protein & Peptide sequence & Variable modification \\
\hline Q96IZ7 & SHSYDR & Phospho(ST) \\
\hline P17252 & STLNPQWNESFTFK & Phospho(ST) \\
\hline Q99700 & GISPVVSEHR & Phospho(ST) \\
\hline Q9P206 & KPSVGVPPPASPSYPR & Phospho(ST) \\
\hline Q9UQ80 & ALLQSSASR & Phospho(ST) \\
\hline Q6QNY0 & VAGEAAETDSEPEPEPEPTAAPR & 2 Phospho(ST) \\
\hline P18206 & SFLDSGYR & Phospho(ST) \\
\hline Q9HCD5 & DPYGFGDSR & Phospho(ST) \\
\hline Q9HCD5 & SSTDSLPGPISR & 2 Phospho(ST) \\
\hline Q9Y2U5 & DRSSPPPGYIPDELHQVAR & Phospho(ST) \\
\hline O95684 & GPTTGEGALDLSDVHSPPKSPEGK & 2 Phospho(ST) \\
\hline Q14934 & FGLGSPLPSPR & 2 Phospho(ST) \\
\hline P18754 & SPPADAIPK & Phospho(ST) \\
\hline P18754 & RSPPADAIPK & Phospho(ST) \\
\hline P18754 & RRSPPADAIPK & Phospho(ST) \\
\hline Q86U90 & LFRPPSPAPAAPGAR & Phospho(ST) \\
\hline Q13242 & STSYGYSR & Phospho(ST) \\
\hline Q13242 & GSPHYFSPFRPY & Phospho(ST) \\
\hline Q9Y519 & TLLLSSDDEF & Phospho(ST) \\
\hline Q9BZZ5 & ASEDTTSGSPPKK & Phospho(ST) \\
\hline Q9NRZ9 & ILENSEDSSPECLF & Phospho(ST) \\
\hline Q99460 & TSSAFVGKTPEASPEPK & 2 Phospho(ST) \\
\hline P52594 & GTPSQSPVVGR & 2 Phospho(ST) \\
\hline Q8NI08 & LSSSSPGATVSPSSSDAEYDKLPDADLAR & 2 Phospho(ST) \\
\hline Q8NDI1 & DLSTSPKPSPIPSPVLGR & 3 Phospho(ST) \\
\hline P42677 & DLLHPSPEEEKR & Phospho(ST) \\
\hline P32519 & YADSPGASSPEQPK & Phospho(ST) \\
\hline Q6VMQ6 & HEHPPNPPVSPGK & Phospho(ST) \\
\hline Q6VMQ6 & NKQDDDLNCEPLSPHNITPEPVSK & Phospho(ST) \\
\hline O00159 & DVESPSWR & Phospho(ST) \\
\hline Q8WUZ0 & $\begin{array}{l}\text { GTEPSPGGTPQPSRPVSPAGPPEGVPEEAQP } \\
\text { PR }\end{array}$ & 4 Phospho(ST) \\
\hline Q9UPS8 & TLFEDRDSDSQDEVVVESLPTTSIK & 2 Phospho(ST) \\
\hline P50613 & SFGSPNR & Phospho(ST) \\
\hline P54259 & TEQELPRPQSPSDLDSLDGR & 2 Phospho(ST) \\
\hline Q8N3F8 & SLHPWYGITPTSSPK & 2 Phospho(ST) \\
\hline P49674 & IQPAGNTSPR & Phospho(ST) \\
\hline Q86U06 & EKSPVREPVDNLSPEER & Phospho(ST) \\
\hline Q92504 & EKQSSEEEEKETR & 2 Phospho(ST) \\
\hline Q15717 & NVALLSQLYHSPAR & Phospho(ST) \\
\hline Q8N1F8 & RASISEPSDTDPEPR & 3 Phospho(ST) \\
\hline Q9UHB6 & TVSPPIR & Phospho(ST) \\
\hline Q9UHB6 & ETPHSPGVEDAPIAK & Phospho(ST) \\
\hline
\end{tabular}




\begin{tabular}{|c|c|c|}
\hline Protein & Peptide sequence & Variable modification \\
\hline Q9UHB6 & SEVQQPVHPKPLSPDSR & Phospho(ST) \\
\hline Q92974 & LQDSSDPDTGSEEEGSSRLSPPHSPR & 2 Phospho(ST) \\
\hline Q9NQG5 & LSMEDSKSPPPK & Phospho(ST) \\
\hline Q9NQG5 & $\begin{array}{l}\text { TFQQIQEEEDDDYPGSYSPQDPSAGPLLTEE } \\
\text { LIK }\end{array}$ & Phospho(ST) \\
\hline Q5JRA6 & DEPPPASQSTSQDCSQALKQSP & Phospho(ST) \\
\hline Q9NYV6 & EGDVDVSDSDDEDDNLPANFDTCHR & 2 Phospho(ST) \\
\hline Q6PJT7 & DLVQPDKPASPK & Phospho(ST) \\
\hline Q8WUB8 & DDNEDNSNDGTQPSKR & Phospho(ST) \\
\hline O75379 & NLLEDDSDEEEDFFLR & Phospho(ST) \\
\hline Q9Y4H2 & HSSETFSSTTTVTPVSPSFAHNPK & 2 Phospho(ST) \\
\hline Q92581 & RGVGSSPR & 2 Phospho(ST) \\
\hline O43426 & TSPCQSPTISEGPVPSLPIRPSR & 2 Phospho(ST) \\
\hline Q9Y2W2 & EVEEFSEDDDEDDSDDSEAEK & 3 Phospho(ST) \\
\hline Q96CW6 & EFGYDSPHDLDSD & 2 Phospho(ST) \\
\hline Q15637 & TGDLGIPPNPEDRSPSPEPIYNSEGK & 2 Phospho(ST) \\
\hline Q96RK0 & TQSLSALPK & Phospho(ST) \\
\hline Q96CP2 & ASQEPSPKPGTEVIPAAPR & Phospho(ST) \\
\hline O60716 & GSLASLDSLR & 2 Phospho(ST) \\
\hline O60716 & TVQPVAMGPDGLPVDASSVSNNYIQTLGR & Phospho(ST) \\
\hline P35613 & KPEDVLDDDDAGSAPLK & Phospho(ST) \\
\hline P35613 & RKPEDVLDDDDAGSAPLK & Phospho(ST) \\
\hline Q8N4C8 & SQSLQDQPTR & Phospho(ST) \\
\hline Q9H4A3 & DVDDGSGSPHSPHQLSSK & 2 Phospho(ST) \\
\hline O14686 & ALSPVIPLIPR & Phospho(ST) \\
\hline Q71RC2 & DGLNQTTIPVSPPSTTKPSR & Phospho(ST) \\
\hline Q6F5E8 & TDLETSPGAAPR & Phospho(ST) \\
\hline Q9ULH0 & YQKLPSDEDESGTEESDNTPLLK & 2 Phospho(ST) \\
\hline C9JH25 & $\begin{array}{l}\text { NFSGLSPPGETTSTSSASGVSGSLGFLGTTLS } \\
\text { LPPYSLERK }\end{array}$ & 7 Phospho(ST) \\
\hline Q15910 & ESSIIAPAPAEDVDTPPR & Phospho(ST) \\
\hline Q92614 & YSHSYLSDSDTEAK & 2 Phospho(ST) \\
\hline O60231 & LLEDSEESSEETVSR & 3 Phospho(ST) \\
\hline Q9Y3B9 & DWDKESDGPDDSRPESASDSDT & 4 Phospho(ST) \\
\hline Q3B726 & HQEVQDQDPVFQGSDSSGYQSDHK & Phospho(ST) \\
\hline Q05519 & KLSRSPSPR & 3 Phospho(ST) \\
\hline Q14181 & AISTPETPLTK & 2 Phospho(ST) \\
\hline Q8TE77 & SPPGSGASTPVGPWDQAVQR & Phospho(ST) \\
\hline P83369 & SAGAGSPARPPSPR & 2 Phospho(ST) \\
\hline P27540 & SDDEQSSADKER & Phospho(ST) \\
\hline Q9H9J4 & HRPSSPR & 2 Phospho(ST) \\
\hline Q9H9J4 & HQQDSDLSAACSDADLHR & 3 Phospho(ST) \\
\hline Q86U86 & ATSPSSSVSGDFDDGHHSVSTPGPSR & 2 Phospho(ST) \\
\hline
\end{tabular}




\begin{tabular}{|c|c|c|}
\hline Protein & Peptide sequence & Variable modification \\
\hline Q9HCD6 & QIASDSPHASPK & 2 Phospho(ST) \\
\hline Q9HCD6 & YQQETSVSQLPGRPKSPLSK & Phospho(ST) \\
\hline P01106 & KFELLPTPPLSPSR & 2 Phospho(ST) \\
\hline P01106 & KFELLPTPPLSPSRR & 2 Phospho(ST) \\
\hline Q5T5C0 & SSSVTSIDKESR & Phospho(ST) \\
\hline Q15084 & DGELPVEDDIDLSDVELDDLGKDEL & Phospho(ST) \\
\hline Q96BY6 & SQTLPIIR & Phospho(ST) \\
\hline O15357 & DASDGEDEKPPLPPR & Phospho(ST) \\
\hline Q8NCF5 & KTEFLDLDNSPLSPPSPR & Phospho(ST) \\
\hline Q9Y5K3 & ELNVSFINEK & Phospho(ST) \\
\hline P51636 & ADVQLFMDDDSYSHHSGLEYADPEK & 2 Phospho(ST) \\
\hline P35580 & QLHLEGASLELSDDDTESK & Phospho(ST) \\
\hline Q7Z695 & TEMAMLVTQAR & 2 Phospho(ST) \\
\hline Q13123 & LLMTPRAAPTSAPPSK & Phospho(ST) \\
\hline Q92994 & $\begin{array}{l}\text { DGSTEDTASSLCGEEDTEDEELEAAASHLN } \\
\mathrm{K}\end{array}$ & 2 Phospho(ST) \\
\hline P23588 & AFGSGYR & Phospho(Y) \\
\hline P23588 & AASIFGGAKPVDTAAR & Phospho(ST) \\
\hline P23588 & SLENETLNKEEDCHSPTSKPPKPDQPLK & Phospho(ST) \\
\hline Q92621 & LQDSFASETNLDFR & 2 Phospho(ST) \\
\hline Q5VT52 & SATPEPVTDNR & Phospho(ST) \\
\hline Q92785 & ILEPDDFLDDLDDEDYEEDTPK & Phospho(Y) \\
\hline Q9UGV2 & SVTSNQSDGTQESCESPDVLDR & Phospho(ST) \\
\hline Q13614 & SASVVSSDSISTSADNFSPDLR & Phospho(ST) \\
\hline Q12893 & VDSPLPSDKAPTPPGK & Phospho(ST) \\
\hline P02765 & CDSSPDSAEDVRK & Phospho(ST) \\
\hline Q09666 & SSKASLGSLEGEAEAEASSPK & 3 Phospho(ST) \\
\hline Q9H1E3 & DDSHSAEDSEDEKEDHK & 2 Phospho(ST) \\
\hline Q9H1E3 & SGKNSQEDSEDSEDKDVK & 3 Phospho(ST) \\
\hline Q9UQ35 & SRTSQR & Phospho(ST) \\
\hline Q9UQ35 & SRTARR & Phospho(ST) \\
\hline Q9UQ35 & VKPETPPR & Phospho(ST) \\
\hline Q9UQ35 & DTLRTPPR & Phospho(ST) \\
\hline Q9UQ35 & SGMSPEQSR & Phospho(ST) \\
\hline Q9UQ35 & ALPQTPRPR & Phospho(ST) \\
\hline Q9UQ35 & RVPSPTPAPK & Phospho(ST) \\
\hline Q9UQ35 & SRASPATHR & 2 Phospho(ST) \\
\hline Q9UQ35 & TVARTPLGQR & Phospho(ST) \\
\hline Q9UQ35 & SLTRSPPAIR & 3 Phospho(ST) \\
\hline Q9UQ35 & SRSRTPLISR & 3 Phospho(ST) \\
\hline Q9UQ35 & SRTPPSAPSQSR & 2 Phospho(ST) \\
\hline Q9UQ35 & ENSFGSPLEFR & 2 Phospho(ST) \\
\hline Q9UQ35 & RSRSVSPCSNVESR & 3 Phospho(ST) \\
\hline
\end{tabular}




\begin{tabular}{|c|c|c|}
\hline Protein & Peptide sequence & Variable modification \\
\hline Q9UQ35 & LPQSSSSESSPPSPQPTK & Phospho(ST) \\
\hline Q9UQ35 & AGMSSNQSISSPVLDAVPR & Phospho(ST) \\
\hline Q9UQ35 & QGSITSPQANEQSVTPQRR & Phospho(ST) \\
\hline Q9UQ35 & GQSQTSPDHRSDTSSPEVR & 4 Phospho(ST) \\
\hline Q9UQ35 & RGEGDAPFSEPGTTSTQRPSSPETATK & 2 Phospho(ST) \\
\hline Q9UQ35 & HGGSPQPLATTPLSQEPVNPPSEASPTR & 4 Phospho(ST) \\
\hline P62736 & CPETLFQPSFIGMESAGIHETTYNSIMK & 2 Phospho(ST) \\
\hline P07900 & EEKESEDKPEIEDVGSDEEEEK & Phospho(ST) \\
\hline Q9NYF8 & FNDSEGDDTEETEDYR & 2 Phospho(ST) \\
\hline Q9NYF8 & QKFNDSEGDDTEETEDYR & Phospho(ST) \\
\hline Q9NYF8 & AEGEWEDQEALDYFSDKESGK & 2 Phospho(ST) \\
\hline Q9NYF8 & SQEEPKDTFEHDPSESIDEFNK & Phospho(ST) \\
\hline Q9NYF8 & GRAEGEWEDQEALDYFSDKESGK & Phospho(Y) \\
\hline Q9NYF8 & AEGEWEDQEALDYFSDKESGKQK & 2 Phospho(ST) \\
\hline Q13428 & AASAPAKGTPGK & Phospho(ST) \\
\hline Q13428 & VGPATPSAQVGK & Phospho(ST) \\
\hline Q13428 & SPAGPAATPAQAQAASTPR & Phospho(ST) \\
\hline Q13428 & KLGAGEGGEASVSPEKTSTTSK & Phospho(ST) \\
\hline Q13428 & AEKQEDSESSEEESDSEEAAASPAQVK & 4 Phospho(ST) \\
\hline Q13428 & AEKQEDSESSEEESDSEEAAASPAQVK & 5 Phospho(ST) \\
\hline Q8IYB3 & YSPPIQR & Phospho(ST) \\
\hline Q8IYB3 & QRSSPVTK & Phospho(ST) \\
\hline Q8IYB3 & TASPPPPPK & 2 Phospho(ST) \\
\hline Q8IYB3 & LSPSASPPRR & Phospho(ST) \\
\hline Q8IYB3 & AASPSPQSVR & 2 Phospho(ST) \\
\hline Q8IYB3 & RYSPSPPPK & 2 Phospho(ST) \\
\hline Q8IYB3 & TASPPPPPKR & 2 Phospho(ST) \\
\hline Q8IYB3 & RSPSPAPPPR & 2 Phospho(ST) \\
\hline Q8IYB3 & RLSPSASPPR & 2 Phospho(ST) \\
\hline Q8IYB3 & KVELSESEEDKGGK & 2 Phospho(ST) \\
\hline Q9Y2W1 & SSSPPPR & Phospho(ST) \\
\hline Q9Y2W1 & SSFSITR & Phospho(ST) \\
\hline Q9Y2W1 & ERSPALK & Phospho(ST) \\
\hline Q9Y2W1 & SYSPAHNR & 2 Phospho(ST) \\
\hline Q9Y2W1 & NKKSPEIHR & Phospho(ST) \\
\hline Q9Y2W1 & TDSEKPFRGSQSPK & 2 Phospho(ST) \\
\hline Q9Y2W1 & WAHDKFSGEEGEIEDDESGTENREEK & 2 Phospho(ST) \\
\hline Q5VUA4 & RRSPSPR & 2 Phospho(ST) \\
\hline P35579 & DELADEIANSSGK & Phospho(ST) \\
\hline P42167 & LREQGTESRSSTPLPTISSSAENTR & 3 Phospho(ST) \\
\hline P42166 & LREQGTESRSSTPLPTISSSAENTR & 3 Phospho(ST) \\
\hline P06748 & GPSSVEDIK & Phospho(ST) \\
\hline P24534 & YGPADVEDTTGSGATDSKDDDDIDLFGSDD & 2 Phospho(ST) \\
\hline
\end{tabular}




\begin{tabular}{|c|c|c|}
\hline Protein & Peptide sequence & Variable modification \\
\hline & EEESEEAK & \\
\hline Q8WWI1 & ATLSSTSGLDLMSESGEGEISPQR & Phospho(ST) \\
\hline Q8WWI1 & TPNNVVSTPAPSPDASQLASSLSSQK & 2 Phospho(ST) \\
\hline P18858 & AETPTESVSEPEVATK & Phospho(ST) \\
\hline Q96ST2 & EAEDSDSDDNIKR & 2 Phospho(ST) \\
\hline Q96ST2 & TIASDSEEEAGKELSDKK & 2 Phospho(ST) \\
\hline Q96ST2 & GHHVTDSENDEPLNLNASDSESEELHR & 4 Phospho(ST) \\
\hline P55081 & SLAALDALNTDDENDEEEYEAWK & Phospho(ST) \\
\hline P55081 & RPDYAPMESSDEEDEEFQFIK & 2 Phospho(ST) \\
\hline P67809 & NEGSESAPEGQAQQR & 2 Phospho(ST) \\
\hline P67809 & NYQQNYQNSESGEK & Phospho(ST) \\
\hline P67809 & AADPPAENSSAPEAEQGGAE & Phospho(ST) \\
\hline Q9NTI5 & GHTASESDEQQWPEEK & Phospho(ST) \\
\hline Q9NTI5 & AESPESSAIESTQSTPQK & Phospho(ST) \\
\hline Q9NTI5 & AESPESSAIESTQSTPQK & 2 Phospho(ST) \\
\hline P51858 & NSTPSEPGSGR & Phospho(ST) \\
\hline Q9H6F5 & LGGLRPESPESLTSVSR & 2 Phospho(ST) \\
\hline P29692 & ATAPQTQHVSPMR & Phospho(ST) \\
\hline P68363 & DYEEVGVDSVEGEGEEEGEEY & Phospho(ST) \\
\hline Q71U36 & DYEEVGVDSVEGEGEEEGEEY & Phospho(ST) \\
\hline P11388 & YLEESDEDDLF & Phospho(ST) \\
\hline P11388 & KPSTSDDSDSNFEK & 4 Phospho(ST) \\
\hline P11388 & ELKPQKSVVSDLEADDVK & 2 Phospho(ST) \\
\hline Q16637 & GTGQSDDSDIWDDTALIK & Phospho(ST) \\
\hline Q9NTJ3 & RREEGPPPPSPDGASSDAEPEPPSGR & Phospho(ST) \\
\hline Q9UPQ0 & VHGSPLELK & Phospho(ST) \\
\hline Q96JM3 & KPSGSPDLWK & Phospho(ST) \\
\hline Q96JM3 & DNQESSDAELSSSEYIK & 2 Phospho(ST) \\
\hline Q96JM3 & KPSPSESPEPWKPFPAVSPEPR & 3 Phospho(ST) \\
\hline O60841 & VEMYSGSDDDDDFNKLPK & Phospho(ST) \\
\hline O60841 & KQSFDDNDSEELEDKDSK & 2 Phospho(ST) \\
\hline $\mathrm{O} 43823$ & SGDEEFKGEDELCDSGR & Phospho(ST) \\
\hline O00567 & SFSKEELMSSDLEETAGSTSIPK & Phospho(ST) \\
\hline O00567 & SFSKEELMSSDLEETAGSTSIPK & 2 Phospho(ST) \\
\hline P10412 & KAPKSPAK & Phospho(ST) \\
\hline P10412 & SETAPAAPAAPAPAEKTPVK & Phospho(ST) \\
\hline P16403 & VAKSPKK & Phospho(ST) \\
\hline P16401 & VAKSPKK & Phospho(ST) \\
\hline P16401 & ATKSPAKPK & Phospho(ST) \\
\hline O43719 & LFEESDDKEDEDADGK & Phospho(ST) \\
\hline O43719 & LFEDDDSNEKLFDEEEDSSEKLFDDSDER & 2 Phospho(ST) \\
\hline Q12888 & GGPGKLSPR & Phospho(ST) \\
\hline Q12888 & SDSPEIPFQAAAGPSDGLDASSPGNSFVGLR & Phospho(ST) \\
\hline
\end{tabular}




\begin{tabular}{|c|c|c|}
\hline Protein & Peptide sequence & Variable modification \\
\hline Q12888 & SDSPEIPFQAAAGPSDGLDASSPGNSFVGLR & 2 Phospho(ST) \\
\hline Q12888 & $\begin{array}{l}\text { TVSSDGCSTPSREEGGCSLASTPATTLHLLQ } \\
\text { LSGQR }\end{array}$ & 5 Phospho(ST) \\
\hline P27816 & SPSTLLPK & Phospho(ST) \\
\hline P27816 & SKVGSTENIK & Phospho(ST) \\
\hline P27816 & LATNTSAPDLK & Phospho(ST) \\
\hline P27816 & DMESPTKLDVTLAK & Phospho(ST) \\
\hline Q9Y3T9 & DLFDLNSSEEDDTEGFSER & Phospho(ST) \\
\hline P20810 & DTSQSDKDLDDALDK & Phospho(ST) \\
\hline P20810 & $\begin{array}{l}\text { EGITGPPADSSKPIGPDDAIDALSSDFTCGSP } \\
\text { TAAGK }\end{array}$ & Phospho(ST) \\
\hline Q5T200 & LISDSVER & Phospho(ST) \\
\hline Q5T200 & RSSPESDR & Phospho(ST) \\
\hline Q5T200 & GDSDISDEEAAQQSK & 2 Phospho(ST) \\
\hline Q5T200 & GPRTPSPPPPIPEDIALGK & 2 Phospho(ST) \\
\hline Q5T200 & KGPRTPSPPPPIPEDIALGK & 2 Phospho(ST) \\
\hline Q5T200 & ERTPESEEENVEWETNR & 2 Phospho(ST) \\
\hline Q5JTV8 & SRDSDESGDKTTR & 2 Phospho(ST) \\
\hline Q5JTV8 & DSHSSEEDEASSQTDLSQTISKK & 2 Phospho(ST) \\
\hline Q5JTV8 & RGLRDSHSSEEDEASSQTDLSQTISK & 3 Phospho(ST) \\
\hline P0DMV8 & GGSGSGPTIEEVD & Phospho(ST) \\
\hline O00566 & KDDNDEEEEDIDFFEDIDSDEDEGGLFGSK & Phospho(ST) \\
\hline P80723 & SDGAPASDSKPGSSEAAPSSK & Phospho(ST) \\
\hline P49736 & RGNDPLTSSPGR & 2 Phospho(ST) \\
\hline Q8WVC0 & YVISDEEEEDDD & Phospho(ST) \\
\hline Q8WVC0 & IYSSDSDEGSEEDKAQR & 3 Phospho(ST) \\
\hline P27824 & QKSDAEEDGGTVSQEEEDRKPK & 2 Phospho(ST) \\
\hline P46821 & VASPKKK & Phospho(ST) \\
\hline P46821 & ESSPLYSPTFSDSTSAVK & Phospho(ST) \\
\hline P46821 & EVPSKEEPSPVKAEVAEK & Phospho(ST) \\
\hline P46821 & KLGDVSPTQIDVSQFGSFK & Phospho(ST) \\
\hline P46821 & DSISAVSSEKVSPSKSPSLSPSPPSPLEK & 3 Phospho(ST) \\
\hline P46821 & VSAEAEVAPVSPEVTQEVVEEHCASPEDK & Phospho(ST) \\
\hline P46821 & DSISAVSSEKVSPSKSPSLSPSPPSPLEK & 4 Phospho(ST) \\
\hline Q8WXI9 & GRLTPSPDIIVLSDNEASSPR & 2 Phospho(ST) \\
\hline P49792 & EDALDDSVSSSSVHASPLASSPVRK & Phospho(ST) \\
\hline Q7Z3J3 & EDALDDSVSSSSVHASPLASSPVRK & Phospho(ST) \\
\hline A6NKT7 & EDALDDSVSSSSVHASPLASSPVRK & Phospho(ST) \\
\hline O14715 & EDALDDSVSSSSVHASPLASSPVRK & Phospho(ST) \\
\hline P35221 & SRTSVQTEDDQLIAGQSAR & 3 Phospho(ST) \\
\hline P26232 & SRTSVQTEDDQLIAGQSAR & 3 Phospho(ST) \\
\hline P11717 & LVSFHDDSDEDLLHI & 2 Phospho(ST) \\
\hline P11717 & ALSSLHGDDQDSEDEVLTIPEVK & 2 Phospho(ST) \\
\hline
\end{tabular}




\begin{tabular}{|c|c|c|}
\hline Protein & Peptide sequence & Variable modification \\
\hline Q15149 & SLQEEHVAVAQLR & Phospho(ST) \\
\hline Q15149 & GYYSPYSVSGSGSTAGSR & Phospho(ST) \\
\hline Q15149 & SSSVGSSSSYPISPAVSR & 2 Phospho(ST) \\
\hline P52756 & GLVAAYSGDSDNEEELVER & Phospho(ST) \\
\hline P22059 & TGSNISGASSDISLDEQYK & Phospho(ST) \\
\hline Q14103 & HSEAATAQR & Phospho(ST) \\
\hline Q14103 & IDASKNEEDEGHSNSSPR & 2 Phospho(ST) \\
\hline Q1KMD3 & EEDEPEERSGDETPGSEVPGDK & 2 Phospho(ST) \\
\hline Q1KMD3 & REEDEPEERSGDETPGSEVPGDK & Phospho(ST) \\
\hline Q99590 & RPQSPSPR & Phospho(ST) \\
\hline Q99590 & SSSNDSVDEETAESDTSPVLEK & 2 Phospho(ST) \\
\hline Q02952 & RPSESDKEDELDK & 2 Phospho(ST) \\
\hline Q02952 & $\begin{array}{l}\text { LSAEYEKVELPSEEQVSGSQGPSEEKPAPLA } \\
\text { TEVFDEK }\end{array}$ & Phospho(ST) \\
\hline Q9H3N1 & KVEEEQEADEEDVSEEEAESK & Phospho(ST) \\
\hline Q6PKG0 & KNTFTAWSDEESDYEIDDR & Phospho(ST) \\
\hline Q6PKG0 & $\begin{array}{l}\text { ESPRPLQLPGAEGPAISDGEEGGGEPGAGG } \\
\text { GAAGAAGAGR }\end{array}$ & Phospho(ST) \\
\hline P19338 & AAVTPGKK & Phospho(ST) \\
\hline P19338 & EVEEDSEDEEMSEDEEDDSSGEEVVIPQKK & 3 Phospho(ST) \\
\hline Q15276 & AQSTDSLGTSGSLQSK & 2 Phospho(ST) \\
\hline P29966 & LSGFSFKK & Phospho(ST) \\
\hline P29966 & AEDGATPSPSNETPKK & Phospho(ST) \\
\hline P17812 & DTYSDRSGSSSPDSEITELK & 2 Phospho(ST) \\
\hline P17812 & DTYSDRSGSSSPDSEITELKFPSINHD & 2 Phospho(ST) \\
\hline Q9Y2X3 & EEPLSEEEPCTSTAIASPEK & 2 Phospho(ST) \\
\hline Q13435 & GFEEEHKDSDDDSSDDEQEK & 3 Phospho(ST) \\
\hline Q9C0C2 & GWSQEGPVK & Phospho(ST) \\
\hline Q9C0C2 & GSGGLFSPSTAHVPDGALGQR & Phospho(ST) \\
\hline Q9C0C2 & LDSPPPSPITEASEAAEAAEAGNLAVSSR & 2 Phospho(ST) \\
\hline Q9UQ88 & DLLSDLQDISDSER & Phospho(ST) \\
\hline Q14676 & SQTTTERDSDTDVEEEELPVENR & 2 Phospho(ST) \\
\hline Q14676 & SQPPGEDSDTDVDDDSRPPGRPAEVHLER & 2 Phospho(ST) \\
\hline Q9Y6X9 & SVAVSDEEEVEEEAER & Phospho(ST) \\
\hline Q9UQE7 & GDVEGSQSQDEGEGSGESER & Phospho(ST) \\
\hline Q12789 & KNSSTDQGSDEEGSLQK & 2 Phospho(ST) \\
\hline Q9BPX3 & LNLAQFLNEDLS & Phospho(ST) \\
\hline Q04726 & YDSDGDKSDDLVVDVSNEDPATPR & 2 Phospho(ST) \\
\hline Q76FK4 & NSSPGEASLLEK & Phospho(ST) \\
\hline Q7Z460 & NSSNTSVGSPSNTIGR & Phospho(ST) \\
\hline P07910 & NDKSEEEQSSSSVK & Phospho(ST) \\
\hline Q96CX2 & SPSGGAAGPLLTPSQSLDGSRR & Phospho(ST) \\
\hline Q5VT52 & GNEPGSDRSPSPSK & 2 Phospho(ST) \\
\hline
\end{tabular}




\begin{tabular}{|c|c|c|}
\hline Protein & Peptide sequence & Variable modification \\
\hline Q5VT52 & SFNYSPNSSTSEVSSTSASK & Phospho(ST) \\
\hline Q9BWU0 & NWEDEDFYDSDDDTFLDR & Phospho(ST) \\
\hline P35269 & KAPTPQEK & Phospho(ST) \\
\hline Q8IVT2 & NALFPEVFSPTPDENSDQNSR & Phospho(ST) \\
\hline Q4KMP7 & TEEARPSPAPGPGTPTGTPTR & 2 Phospho(ST) \\
\hline Q9BW71 & EVSDSEAGGGPQGER & 2 Phospho(ST) \\
\hline Q9BW71 & EESEESEAEPVQR & 2 Phospho(ST) \\
\hline Q96N67 & SLSNSNPDISGTPTSPDDEVR & Phospho(ST) \\
\hline O60343 & LGSVDSFER & 2 Phospho(ST) \\
\hline O60343 & SLTSSLENIFSR & 2 Phospho(ST) \\
\hline O60343 & TSSTCSNESLSVGGTSVTPR & 2 Phospho(ST) \\
\hline O60343 & KRTSSTCSNESLSVGGTSVTPR & 4 Phospho(ST) \\
\hline P46013 & RPQTPK & Phospho(ST) \\
\hline P46013 & AFKESPK & Phospho(ST) \\
\hline P46013 & DINTFVGTPVEK & Phospho(ST) \\
\hline P20700 & VTVSRASSSR & 2 Phospho(ST) \\
\hline P20700 & LKLSPSPSSR & 2 Phospho(ST) \\
\hline P20700 & AGGPTTPLSPTR & Phospho(ST) \\
\hline P20700 & AGGPTTPLSPTR & 2 Phospho(ST) \\
\hline Q03252 & LKLSPSPSSR & 2 Phospho(ST) \\
\hline O60716 & GSLASLDSLR & Phospho(ST) \\
\hline O60716 & GSLASLDSLRK & 2 Phospho(ST) \\
\hline O60716 & HYEDGYPGGSDNYGSLSR & Phospho(ST) \\
\hline Q08J23 & ESTQLSPADLTEGKPTDPSK & Phospho(ST) \\
\hline Q9H4G0 & LPSSPASPSPK & Phospho(ST) \\
\hline Q9H4G0 & GAPSQDDESGGIEDSPDR & Phospho(ST) \\
\hline Q9H4G0 & DEDGESGGQRSEAEEGEVR & 2 Phospho(ST) \\
\hline Q96T58 & SPSGSGSRSR & Phospho(ST) \\
\hline Q96T58 & SQSPVHLR & 2 Phospho(ST) \\
\hline Q96T58 & DSELKTPPSVGPPSVTVVTLESAPSALEK & 2 Phospho(ST) \\
\hline Q14978 & LQTPNTFPK & 2 Phospho(ST) \\
\hline Q14978 & VAGGAAPSKPASAK & Phospho(ST) \\
\hline Q14978 & AASSSSSSSSSSSSDDSEEEK & Phospho(ST) \\
\hline Q14978 & KASSSDSEDSSEEEEEVQGPPAK & 6 Phospho(ST) \\
\hline Q96B36 & SSDEENGPPSSPDLDR & 2 Phospho(ST) \\
\hline O95218 & YNLDASEEEDSNK & Phospho(ST) \\
\hline O95218 & ESEGEEEDEDEDLSK & Phospho(ST) \\
\hline O95218 & ENVEYIEREESDGEYDEFGRK & Phospho(ST) \\
\hline O75122 & LSVARSSR & Phospho(ST) \\
\hline Q7Z5L9 & RPASVSSSAAVEHEQR & Phospho(ST) \\
\hline Q6NXS1 & IQEQESSGEEDSDLSPEER & 2 Phospho(ST) \\
\hline Q13283 & TFSWASVTSK & Phospho(ST) \\
\hline Q96T23 & IESDEEEDFENVGK & Phospho(ST) \\
\hline
\end{tabular}




\begin{tabular}{|c|c|c|}
\hline Protein & Peptide sequence & Variable modification \\
\hline Q96T23 & TFLDKDAQRLSPIPEEVPK & Phospho(ST) \\
\hline Q96T23 & IETDEEESCDNAHGDANQPAR & Phospho(ST) \\
\hline Q96T23 & GRSTDEYSEADEEEEEEEGKPSR & 3 Phospho(ST) \\
\hline Q7Z4V5 & GSGGSSGDELREDDEPVK & 3 Phospho(ST) \\
\hline O43815 & KTWNPK & Phospho(ST) \\
\hline O75592 & VNSGDTEVGSSLLR & Phospho(ST) \\
\hline O75592 & SLSPNHNTLQTLK & Phospho(ST) \\
\hline Q13425 & KPSLVSDLPWEGAAPQSPSFSGSEDSGSPK & Phospho(ST) \\
\hline Q9NZ63 & RGDSESEEDEQDSEEVR & 2 Phospho(ST) \\
\hline P04075 & GILAADESTGSIAK & Phospho(ST) \\
\hline P13639 & AGETRFTDTR & Phospho(ST) \\
\hline Q02880 & ASGSENEGDYNPGR & Phospho(ST) \\
\hline Q02880 & FDSNEEDSASVFSPSFGLK & 2 Phospho(ST) \\
\hline Q13185 & KSLSDSESDDSK & Phospho(ST) \\
\hline Q13185 & LTWHSCPEDEAQ & Phospho(ST) \\
\hline Q9UHB6 & QWTSLSLR & Phospho(ST) \\
\hline Q7Z422 & SPPKVPIVIQDDSLPAGPPPQIR & Phospho(ST) \\
\hline P28715 & NAPAAVDEGSISPR & Phospho(ST) \\
\hline O94804 & QVAEQGGDLSPAANR & Phospho(ST) \\
\hline O94804 & ASQSRPNSSALETLGGEK & 2 Phospho(ST) \\
\hline Q02241 & SSTVAPAQPDGAESEWTDVETR & Phospho(ST) \\
\hline Q9P2B4 & DLSPTLIDNSAAK & Phospho(ST) \\
\hline Q8WVB6 & GDAASSPAPAASVGSSQGGAR & Phospho(ST) \\
\hline Q8WVB6 & VENSPQVDGSPPGLEGLLGGIGEK & Phospho(ST) \\
\hline P16949 & SHEAEVLK & Phospho(ST) \\
\hline P16949 & ASGQAFELILSPR & Phospho(ST) \\
\hline P52948 & NLNNSNLFSPVNR & 2 Phospho(ST) \\
\hline Q9BVS4 & SSGDPEQIKEDSLSEESADAR & 3 Phospho(ST) \\
\hline Q13523 & KPIKSPSK & Phospho(ST) \\
\hline Q13523 & ARSPTDDK & Phospho(ST) \\
\hline Q13523 & RSKSQDQAR & Phospho(ST) \\
\hline Q5JTD0 & GSPEEELPLPAFEK & Phospho(ST) \\
\hline Q5JTD0 & KDSLTQAQEQGNLLN & Phospho(ST) \\
\hline Q8N9T8 & AFVEDSEDEDGAGEGGSSLLQK & Phospho(ST) \\
\hline Q9Y2X7 & SQSDLDDQHDYDSVASDEDTDQEPLR & 3 Phospho(ST) \\
\hline Q9BVG4 & RADSGEEENTK & Phospho(ST) \\
\hline Q9BVG4 & GADSGEEKEEGINR & Phospho(ST) \\
\hline Q53F19 & AEAPAGPALGLPSPEAESGVDR & Phospho(ST) \\
\hline Q53F19 & $\begin{array}{l}\text { DKQEDSSDDDEAEEGEVEDENSSDVELDTL } \\
\text { SQVEEESLLR }\end{array}$ & 3 Phospho(ST) \\
\hline Q14157 & NPSDSAVHSPFTK & Phospho(ST) \\
\hline Q14157 & SPAVATSTAAPPPPSSPLPSK & Phospho(ST) \\
\hline P49959 & GVDFESSEDDDDDPFMNTSSLR & 2 Phospho(ST) \\
\hline
\end{tabular}




\begin{tabular}{|c|c|c|}
\hline Protein & Peptide sequence & Variable modification \\
\hline Q641Q2 & TPSDDEEDNLFAPPK & Phospho(ST) \\
\hline Q9Y4E1 & TPSDDEEDNLFAPPK & Phospho(ST) \\
\hline O76021 & AAESETPGKSPEKKPK & Phospho(ST) \\
\hline Q99733 & REFITGDVEPTDAESEWHSENEEEEK & Phospho(ST) \\
\hline Q9C0C9 & LIHGEDSDSEGEEEGR & 2 Phospho(ST) \\
\hline P02765 & CDSSPDSAEDVR & Phospho(ST) \\
\hline P51991 & SSGSPYGGGYGSGGGSGGYGSR & Phospho(ST) \\
\hline Q9NWH9 & ERASLDK & Phospho(ST) \\
\hline Q9NWH9 & DGQDAIAQSPEKESK & Phospho(ST) \\
\hline Q9Y5J1 & KTSSDDESEEDEDDLLQR & 3 Phospho(ST) \\
\hline B5ME19 & QTALASR & Phospho(ST) \\
\hline B5ME19 & QPLLLSEDEEDTKR & Phospho(ST) \\
\hline Q92522 & AGGSAALSPSKK & Phospho(ST) \\
\hline Q15154 & KDEETEESEYDSEHENSEPVTNIR & 3 Phospho(ST) \\
\hline Q9UMZ2 & QLSLEGSGLGVEDLK & Phospho(ST) \\
\hline P23588 & AFGSGYR & Phospho(ST) \\
\hline P23588 & ESEKSLENETLNK & Phospho(ST) \\
\hline P23588 & YAALSVDGEDENEGEDYAE & Phospho(ST) \\
\hline O60293 & LDSSPVSSPR & Phospho(ST) \\
\hline Q9Y2U8 & VLLGFSSDESDVEASPR & Phospho(ST) \\
\hline Q9Y2U8 & VLLGFSSDESDVEASPR & 2 Phospho(ST) \\
\hline Q9Y2U8 & ENYSDSEEEDDDDVASSR & 2 Phospho(ST) \\
\hline Q96C19 & RADLNQGIGEPQSPSR & Phospho(ST) \\
\hline P53396 & TASFSESR & Phospho(ST) \\
\hline P34947 & DVLDIEQFSTVK & 2 Phospho(ST) \\
\hline P25205 & RSEDESETEDEEEKSQEDQEQK & 2 Phospho(ST) \\
\hline Q6KC79 & SRPDSPR & Phospho(ST) \\
\hline Q99549 & LSLNNDIFEANSDSDQQSETK & 2 Phospho(ST) \\
\hline Q5QJE6 & QILIACSPVSSVR & Phospho(ST) \\
\hline Q3YEC7 & ADDFPVRDDPSDVTDEDEGPAEPPPPPK & 2 Phospho(ST) \\
\hline Q3YEC7 & RADDFPVRDDPSDVTDEDEGPAEPPPPPK & 2 Phospho(ST) \\
\hline Q5T8D3 & AESSDSGAESEEEEAQEEVK & 2 Phospho(ST) \\
\hline Q8IY81 & GTEASSGTEAATGLEGEEK & Phospho(ST) \\
\hline O75400 & QETVADFTPK & Phospho(ST) \\
\hline O75400 & DSGNWDTSGSELSEGELEK & 4 Phospho(ST) \\
\hline Q8WX93 & GVTPAGFPK & Phospho(ST) \\
\hline Q8WX93 & SPSGHPHVR & Phospho(ST) \\
\hline Q92769 & IACDEEFSDSEDEGEGGRR & 2 Phospho(ST) \\
\hline Q9UKV3 & ISEDETER & Phospho(ST) \\
\hline Q9UKV3 & LQPERGSPK & Phospho(ST) \\
\hline Q9UKV3 & AESPAEKVPEESVLPLVQK & Phospho(ST) \\
\hline Q01082 & GEQVSQNGLPAEQGSPR & Phospho(ST) \\
\hline Q13112 & TDTPPSSVPTSVISTPSTEEIQSETPGDAQGSP & Phospho(ST) \\
\hline
\end{tabular}




\begin{tabular}{|c|c|c|}
\hline Protein & Peptide sequence & Variable modification \\
\hline & PELK & \\
\hline P51532 & IEKEDDSEGEESEEEEEGEEEGSESESR & 3 Phospho(ST) \\
\hline Q9NYM9 & AQSPGAVEEILDR & Phospho(ST) \\
\hline Q6L8Q7 & $\begin{array}{l}\text { EAKPGAAEPEVGVPSSLSPSSPSSSWTETDV } \\
\text { EER }\end{array}$ & Phospho(ST) \\
\hline Q92688 & KRETDDEGEDD & Phospho(ST) \\
\hline P12270 & AADSQNSGEGNTGAAESSFSQEVSR & Phospho(ST) \\
\hline O14974 & STGVSFWTQDSDENEQEQQSDTEEGSNKK & 2 Phospho(ST) \\
\hline Q8IU81 & KASPEPEGEAAGK & Phospho(ST) \\
\hline Q9HCD6 & DCSYGAVTSPTSTLESR & Phospho(ST) \\
\hline Q9Y5K6 & SVDFDSLTVR & Phospho(ST) \\
\hline Q8NEY8 & DTSPSSGSAVSSSK & Phospho(ST) \\
\hline Q15651 & AEEAQKTESVDNEGE & Phospho(ST) \\
\hline Q9UIG0 & LAEDEGDSEPEAVGQSR & Phospho(ST) \\
\hline Q13573 & GPPSPPAPVMHSPSR & 2 Phospho(ST) \\
\hline P51531 & GRPPAEKLSPNPPK & Phospho(ST) \\
\hline P51531 & AKPVVSDFDSDEEQDER & Phospho(ST) \\
\hline P51531 & AKPVVSDFDSDEEQDER & 2 Phospho(ST) \\
\hline Q9H8G2 & NCSIEEIKK & Phospho(ST) \\
\hline Q9H8G2 & STDSSSVSGSLQQETK & Phospho(ST) \\
\hline O95251 & SQQQPTPVTPK & Phospho(ST) \\
\hline O95251 & LSQSSQDSSPVR & Phospho(ST) \\
\hline O95251 & SSGSETEQVVDFSDR & Phospho(ST) \\
\hline Q9BXY0 & $\begin{array}{l}\text { ALEQQEAESDSSDTEEKDDDDDDEEDVGK } \\
\mathrm{R}\end{array}$ & 2 Phospho(ST) \\
\hline Q9BXY0 & $\begin{array}{l}\text { ALEQQEAESDSSDTEEKDDDDDDEEDVGK } \\
\mathrm{R}\end{array}$ & 3 Phospho(ST) \\
\hline Q96BT3 & ALLETASPR & Phospho(ST) \\
\hline Q86VM9 & LGVSVSPSR & Phospho(ST) \\
\hline Q86VM9 & LGVSVSPSR & 2 Phospho(ST) \\
\hline Q86VM9 & $\begin{array}{l}\text { GPSQEEEDNHSDEEDRASEPKSQDQDSEVN } \\
\text { ELSR }\end{array}$ & 3 Phospho(ST) \\
\hline O15027 & AQQELVPPQQQASPPQLPK & Phospho(ST) \\
\hline O15027 & GSVSQPSTPSPPKPTGIFQTSANSSFEPVK & 4 Phospho(ST) \\
\hline Q07666 & SRGGGGGSRGGAR & Phospho(ST) \\
\hline Q66K74 & SPAEGSERLSLSPLR & 2 Phospho(ST) \\
\hline Q66K74 & ALELPLAASSIPRPRTPSPESHR & 2 Phospho(ST) \\
\hline Q7KZ85 & TRTPASINATPANINLADLTR & 2 Phospho(ST) \\
\hline Q7KZ85 & $\begin{array}{l}\text { GFINDDDDEDEGEEDEGSDSGDSEDDVGH } \\
\mathrm{K}\end{array}$ & 3 Phospho(ST) \\
\hline P50502 & ADEPSSEESDLEIDK & 3 Phospho(ST) \\
\hline Q8IZP2 & ADEPSSEESDLEIDK & 3 Phospho(ST) \\
\hline P98175 & HRHSPTGPPGFPR & Phospho(ST) \\
\hline
\end{tabular}




\begin{tabular}{|c|c|c|}
\hline Protein & Peptide sequence & Variable modification \\
\hline P98175 & GLVAAYSGESDSEEEQER & 2 Phospho(ST) \\
\hline Q86VQ1 & GPSPSSPTPPAAAAPAEQAPR & 2 Phospho(ST) \\
\hline Q06210 & VDSTTCLFPVEEK & Phospho(ST) \\
\hline Q96QU8 & $\begin{array}{l}\text { HSVTAATPPPSPTSGESGDLLSNLLQSPSSA } \\
\mathrm{K}\end{array}$ & 2 Phospho(ST) \\
\hline Q53H80 & TLDFDPLLSPASPK & 2 Phospho(ST) \\
\hline Q29RF7 & EQSSEAAETGVSENEENPVR & Phospho(ST) \\
\hline Q8NHG8 & SLGGAVGSVASGAR & Phospho(ST) \\
\hline Q9H9C1 & TRPGSFQSLSDALSDTPAK & Phospho(ST) \\
\hline Q6NZI2 & GSSPDVHALLEITEESDAVLVDKSDSD & 3 Phospho(ST) \\
\hline O95453 & KELSPAGSISK & Phospho(ST) \\
\hline P48730 & GAPVNISSSDLTGR & Phospho(ST) \\
\hline P07814 & EYIPGQPPLSQSSDSSPTR & 2 Phospho(ST) \\
\hline P49327 & ADEASELACPTPK & Phospho(ST) \\
\hline Q8NEY1 & SVSSLSNR & Phospho(ST) \\
\hline Q8TAQ2 & KRSPSPSPTPEAK & 3 Phospho(ST) \\
\hline Q9UBC2 & STPSHGSVSSLNSTGSLSPK & Phospho(ST) \\
\hline O14497 & SNSVGIQDAFNDGSDSTFQK & Phospho(ST) \\
\hline Q99081 & GNAAGSSQTGDALGK & Phospho(ST) \\
\hline Q05682 & GNVFSSPTAAGTPNK & Phospho(ST) \\
\hline Q05682 & GNVFSSPTAAGTPNKETAGLK & 2 Phospho(ST) \\
\hline Q05682 & TPDGNKSPAPKPSDLRPGDVSSK & Phospho(ST) \\
\hline Q7Z6E9 & DFTPNR & Phospho(ST) \\
\hline Q86YR5 & APSSDEECFFDLLTK & Phospho(ST) \\
\hline Q03701 & SQLDDHPESDDEENFIDANDDEDMEK & Phospho(ST) \\
\hline Q96NU1 & GPTPGQAPAGGAGAEGK & Phospho(ST) \\
\hline Q96NU1 & LWAQDGSEDEPPKDSDGEDPETAAVGCR & Phospho(ST) \\
\hline Q7Z5K2 & RPESPSEISPIK & 2 Phospho(ST) \\
\hline Q99569 & IGSVTSR & Phospho(ST) \\
\hline Q99569 & VGSPLTLTDAQTR & Phospho(ST) \\
\hline Q9GZR7 & AQAVSEEEEEEEGK & Phospho(ST) \\
\hline Q13501 & SRLTPVSPESSSTEEK & Phospho(ST) \\
\hline Q9H3Q1 & RSQSVTR & Phospho(ST) \\
\hline Q8NEJ9 & LSSEDEEEDEAEDDQSEASGK & 2 Phospho(ST) \\
\hline P16989 & $\begin{array}{l}\text { SPVGSGAPQAAAPAPAAHVAGNPGGDAAP } \\
\text { AATGTAAAASLATAAGSEDAEKK }\end{array}$ & Phospho(ST) \\
\hline Q9UKS6 & DGTAPPPQSPGSPGTGQDEEWSDEESPRK & Phospho(ST) \\
\hline Q96NB3 & EEENADSDDEGELQDLLSQDWR & Phospho(ST) \\
\hline Q96K76 & ETWDTAEEDSGTDSEYDESGK & 2 Phospho(ST) \\
\hline Q7L4I2 & EQSEVSVSPR & 2 Phospho(ST) \\
\hline Q6PK04 & VQAGPGSPR & Phospho(ST) \\
\hline Q6XE24 & GYGFVDFDSPAAAQK & Phospho(ST) \\
\hline Q14315 & LGSFGSITR & Phospho(ST) \\
\hline
\end{tabular}




\begin{tabular}{|c|c|c|}
\hline Protein & Peptide sequence & Variable modification \\
\hline Q15637 & TGDLGIPPNPEDRSPSPEPIYNSEGK & Phospho(ST) \\
\hline Q15637 & TGDLGIPPNPEDRSPSPEPIYNSEGKR & 2 Phospho(ST) \\
\hline Q9H1H9 & TGSCSELDACPSK & Phospho(ST) \\
\hline O95400 & GPGQPSSPQR & Phospho(ST) \\
\hline P20839 & LVGIVTSR & Phospho(ST) \\
\hline Q9Y232 & ILVPKSPVK & Phospho(ST) \\
\hline Q9Y232 & TNRTSPNNAR & Phospho(ST) \\
\hline Q8NDT2 & TFLEGDWTSPSK & Phospho(ST) \\
\hline Q96RK0 & KNSTDLDSAPEDPTSPK & 2 Phospho(ST) \\
\hline Q02447 & $\begin{array}{l}\text { IGPPSPGDDEEEAAAAAGAPAAAGATGDLA } \\
\text { SAQLGGAPNR }\end{array}$ & Phospho(ST) \\
\hline Q5T1M5 & DSGSDGHSVSSR & Phospho(ST) \\
\hline Q5T1M5 & RPSQEQSASASSGQPQAPLNR & Phospho(ST) \\
\hline Q5VTR2 & ALVVPEPEPDSDSNQER & Phospho(ST) \\
\hline P49790 & SGIDITDFQAK & Phospho(ST) \\
\hline Q92766 & ANSGGVDLDSSGEFASIEK & 2 Phospho(ST) \\
\hline Q13541 & $\begin{array}{l}\text { RVVLGDGVQLPPGDYSTTPGGTLFSTTPGG } \\
\text { TR }\end{array}$ & Phospho(ST) \\
\hline Q13541 & $\begin{array}{l}\text { NSPVTKTPPRDLPTIPGVTSPSSDEPPMEAS } \\
\text { QSHLR }\end{array}$ & 2 Phospho(ST) \\
\hline Q96IZ0 & STTSVSEEDVSSR & Phospho(ST) \\
\hline O75179 & NSPLDCGSASPNK & Phospho(ST) \\
\hline O75179 & EHYPVSSPSSPSPPAQPGGVSR & 2 Phospho(ST) \\
\hline Q99958 & VETLSPESALQGSPR & 2 Phospho(ST) \\
\hline Q86TC9 & TPEPSSPVKEPPPVLAKPK & Phospho(ST) \\
\hline Q86TC9 & $\begin{array}{l}\text { EAEQAASEAAGGDTTPGSSPSSLYYEEPLG } \\
\text { QPPR }\end{array}$ & Phospho(ST) \\
\hline Q96CB8 & LEKPETQSSPITVQSSK & Phospho(ST) \\
\hline P01106 & KDSGSPNPAR & Phospho(ST) \\
\hline P01106 & PLNVSFTNR & Phospho(ST) \\
\hline Q96TC7 & SQSLPNSLDYTQTSDPGR & Phospho(ST) \\
\hline P49321 & $\begin{array}{l}\text { GGAAPEGPNEAEVTSGKPEQEVPDAEEEKS } \\
\text { VSGTDVQEECR }\end{array}$ & Phospho(ST) \\
\hline O43159 & ALEAASLSQHPPSLCISDSEEEEEER & 2 Phospho(ST) \\
\hline Q8N3E9 & ALSDREEEEEDDEEEEEEVEAAAQR & Phospho(ST) \\
\hline P0C1Z6 & LLPYPTLASPASD & 2 Phospho(ST) \\
\hline O43395 & GDDDEESDEEAVKK & Phospho(ST) \\
\hline O43395 & WDEQTSNTKGDDDEESDEEAVKK & Phospho(ST) \\
\hline Q13247 & SNSPLPVPPSK & 2 Phospho(ST) \\
\hline Q14137 & IGDEYAEDSSDEEDIR & 2 Phospho(ST) \\
\hline Q92882 & TLSNAEDYLDDEDSD & Phospho(ST) \\
\hline Q5SW79 & SDSEATISR & Phospho(ST) \\
\hline Q5SW79 & SIKSDVPVYLK & 2 Phospho(ST) \\
\hline
\end{tabular}




\begin{tabular}{|c|c|c|}
\hline Protein & Peptide sequence & Variable modification \\
\hline Q5SW79 & TEEDSKSIKSDVPVYLK & 2 Phospho(ST) \\
\hline Q8WUF5 & GRPSSPR & Phospho(ST) \\
\hline P28290 & MPSPFR & Phospho(ST) \\
\hline P28290 & ASVALTPTAPSR & Phospho(ST) \\
\hline P28290 & SQSLPTTLLSPVR & Phospho(ST) \\
\hline Q9H501 & ALAEEASEEELPSDVDLNDPYFAEEVK & 2 Phospho(ST) \\
\hline Q9H501 & GNIETSSEDEDDTADLFPEESGFEHAWR & 3 Phospho(ST) \\
\hline Q9H501 & GKGNIETSSEDEDDTADLFPEESGFEHAWR & 3 Phospho(ST) \\
\hline O75376 & RESPPIR & Phospho(ST) \\
\hline Q9H0D6 & RKAEDSDSEPEPEDNVR & 2 Phospho(ST) \\
\hline P18206 & SLLDASEEAIK & Phospho(ST) \\
\hline P18206 & GWLRDPSASPGDAGEQAIR & Phospho(ST) \\
\hline Q13443 & HVSPVTPPR & Phospho(ST) \\
\hline Q7Z4S6 & KLSSSDAPAQDTGSSAAAVETDASR & Phospho(ST) \\
\hline Q9BUQ8 & RSSLSPGR & 2 Phospho(ST) \\
\hline Q9BUQ8 & DASPSKEER & Phospho(ST) \\
\hline P40222 & $\begin{array}{l}\text { SSPGQPEAGPEGAQERPSQAAPAVEAEGPG } \\
\text { SSQAPR }\end{array}$ & Phospho(ST) \\
\hline Q15361 & YLSADSGDADDSDADLGSAVK & 2 Phospho(ST) \\
\hline Q7Z4H7 & AVLSDSPQLSEGK & Phospho(ST) \\
\hline P29590 & KASPEAASTPR & Phospho(ST) \\
\hline Q96PE2 & SPSFGAGEGLLR & Phospho(ST) \\
\hline Q14160 & ALSPAELR & Phospho(ST) \\
\hline Q14160 & NSLESISSIDR & 3 Phospho(ST) \\
\hline Q6P996 & GSDALSETSSVSHIEDLEK & 2 Phospho(ST) \\
\hline Q2KHR3 & NLEHLSSFSSDEDDPGYSQDAYK & 2 Phospho(ST) \\
\hline Q2KHR3 & GQDTVAIEGFTDEEDTESGGEGQYR & 2 Phospho(ST) \\
\hline Q15836 & ADALQAGASQFETSAAK & Phospho(ST) \\
\hline Q6P1N0 & KQNSPVAPTAQPK & Phospho(ST) \\
\hline O60264 & TPTSPLK & Phospho(ST) \\
\hline Q01167 & FAQSAPGSPLSSQPVLITVQR & Phospho(ST) \\
\hline Q01167 & FAQSAPGSPLSSQPVLITVQR & 2 Phospho(ST) \\
\hline Q96T37 & LKSPSQK & Phospho(ST) \\
\hline P50613 & AYTHQVVTR & Phospho(ST) \\
\hline Q8WXE1 & LAAPSVSHVSPR & Phospho(ST) \\
\hline Q86UE4 & SQEPIPDDQKVSDDDKEK & Phospho(ST) \\
\hline O15231 & GGQGDPAVPAQQPADPSTPER & Phospho(ST) \\
\hline P07948 & GKDSLSDDGVDLK & Phospho(ST) \\
\hline $\mathrm{O} 75533$ & WDETPGR & Phospho(ST) \\
\hline Q96KR1 & RDSDGVDGFEAEGK & Phospho(ST) \\
\hline Q96KR1 & RRDSDGVDGFEAEGK & Phospho(ST) \\
\hline Q15021 & YQPLASTASDNDFVTPEPR & Phospho(ST) \\
\hline Q99547 & HKAEEVEDETVELDVSDEEMAR & Phospho(ST) \\
\hline
\end{tabular}




\begin{tabular}{|c|c|c|}
\hline Protein & Peptide sequence & Variable modification \\
\hline Q9Y2K1 & VTDKDCNESTDNDELEDEPEEPFYR & 2 Phospho(ST) \\
\hline O00512 & IPVEGPLSPSR & Phospho(ST) \\
\hline P78536 & SFEDLTDHPVTR & Phospho(ST) \\
\hline Q9Y2F5 & SSGIEYTK & Phospho(ST) \\
\hline Q6P2E9 & DSQDASAEQSDHDDEVASLASASGGFGTK & Phospho(ST) \\
\hline P51116 & TDGSISGDRQPVTVADYISR & Phospho(ST) \\
\hline Q8IVL1 & YPDVASPTLR & Phospho(ST) \\
\hline Q9UIF9 & GRESPAAGPR & Phospho(ST) \\
\hline Q96Q15 & RDQSTIPR & Phospho(ST) \\
\hline Q96Q15 & NLATSADTPPSTVPGTGK & 2 Phospho(ST) \\
\hline O00559 & KLSGDQITLPTTVDYSSVPK & Phospho(ST) \\
\hline Q8TBZ6 & NELDSPHEEK & Phospho(ST) \\
\hline Q07065 & GGHGAASPSEK & Phospho(ST) \\
\hline Q13242 & GRDSPYQSR & Phospho(ST) \\
\hline P13051 & KAPAGQEEPGTPPSSPLSAEQLDR & 2 Phospho(ST) \\
\hline P52272 & GNFGGSFAGSFGGAGGHAPGVAR & Phospho(ST) \\
\hline P08621 & $\begin{array}{l}\text { GGGGDMAEPSEAGDAPPDDGPPGELGPDG } \\
\text { PDGPEEK }\end{array}$ & Phospho(ST) \\
\hline Q9BTA9 & DAGDPSPPNK & Phospho(ST) \\
\hline Q53GS9 & EREVDEDSEPER & Phospho(ST) \\
\hline Q13177 & YLSFTPPEKDGFPSGTPALNAK & Phospho(ST) \\
\hline Q9NQW6 & TPISPLK & Phospho(ST) \\
\hline Q9NQW6 & SCTKPSPSK & Phospho(ST) \\
\hline O95684 & EKGPTTGEGALDLSDVHSPPKSPEGK & 2 Phospho(ST) \\
\hline P18887 & TKPTQAAGPSSPQKPPTPEETK & 2 Phospho(ST) \\
\hline P46100 & LTPVSLSNSPIK & 2 Phospho(ST) \\
\hline P46100 & $\begin{array}{l}\text { ATSSSNPSSPAPDWYKDFVTDADAEVLEHS } \\
\text { GK }\end{array}$ & 2 Phospho(ST) \\
\hline Q66K14 & $\begin{array}{l}\text { KASVVDPSTESSPAPQEGSEQPASPASPLSS } \\
\mathrm{R}\end{array}$ & Phospho(ST) \\
\hline Q9UPN4 & KEEAVSSLR & Phospho(ST) \\
\hline Q13131 & TSCGSPNYAAPEVISGR & Phospho(ST) \\
\hline Q9Y2K7 & YSLTELNMAGCNK & Phospho(Y) \\
\hline Q9Y2K7 & $\begin{array}{l}\text { TPQRGDEEGLGGEEEEEEEEEEEDDSAEEG } \\
\text { GAAR }\end{array}$ & Phospho(ST) \\
\hline P35658 & SPGSTPTTPTSSQAPQK & Phospho(ST) \\
\hline Q6UN15 & AESPDLR & Phospho(ST) \\
\hline Q6UN15 & ERDHSPTPSVFNSDEER & 2 Phospho(ST) \\
\hline Q5PRF9 & QGSDEWGGPAELGPGEAGPGWQDKPPR & Phospho(ST) \\
\hline Q6ZNJ1 & $\begin{array}{l}\text { SQSVPASTGLGWGSGLVAPLQEGSIDSTLA } \\
\text { GTQDTR }\end{array}$ & Phospho(ST) \\
\hline Q9UHF7 & GSSERGSPIEK & Phospho(ST) \\
\hline Q9UHF7 & ATEETGQAQSGQANCQGLSPVSVASK & Phospho(ST) \\
\hline
\end{tabular}




\begin{tabular}{|c|c|c|}
\hline Protein & Peptide sequence & Variable modification \\
\hline Q14181 & SPHQLLSPSSFSPSATPSQK & Phospho(ST) \\
\hline Q7L1Q6 & NAEEESESEAEEGD & Phospho(ST) \\
\hline O14979 & VFVGGLSPDTSEEQIK & Phospho(ST) \\
\hline Q01130 & SRSPPPVSK & 2 Phospho(ST) \\
\hline O00470 & EGGSKSDSEDITR & Phospho(ST) \\
\hline Q9UBK8 & SSRGQEEISGALPVASPASSR & Phospho(ST) \\
\hline P01891 & KGGSYSQAASSDSAQGSDVSLTACKV & 2 Phospho(ST) \\
\hline Q13200 & APVQPQQSPAAAPGGTDEKPSGK & Phospho(ST) \\
\hline Q9H9L4 & ILDEDSWSDGEQEPITVDQTWR & 2 Phospho(ST) \\
\hline P15056 & SASEPSLNR & Phospho(ST) \\
\hline $\mathrm{P} 15056$ & RDSSDDWEIPDGQITVGQR & Phospho(ST) \\
\hline Q8TBF4 & STYFSDEEELSD & 2 Phospho(ST) \\
\hline Q00536 & RLSLPADIR & Phospho(ST) \\
\hline Q00536 & RVSLSEIGFGK & Phospho(ST) \\
\hline Q00537 & RLSLPADIR & Phospho(ST) \\
\hline Q9NYL2 & NFSSLHLNSR & Phospho(ST) \\
\hline Q9NYL2 & YQQITPVNQSR & Phospho(ST) \\
\hline Q9HCE3 & KSPSPVK & Phospho(ST) \\
\hline Q9HCE3 & AADKSPESQNLIDGTK & Phospho(ST) \\
\hline Q13464 & STANQSFR & Phospho(ST) \\
\hline Q9BXP5 & ERFSPPR & Phospho(ST) \\
\hline Q9Y3D3 & TDAEATDTEATET & Phospho(ST) \\
\hline Q96SB4 & $\begin{array}{l}\text { GSAPHSESDLPEQEEEILGSDDDEQEDPNDY } \\
\text { CK }\end{array}$ & 3 Phospho(ST) \\
\hline A6NKF1 & TLEEVVMAEEEDEGTDRPGSPA & Phospho(ST) \\
\hline Q8IVT5 & ALHSFITPPTTPQLR & 2 Phospho(ST) \\
\hline Q8IVT5 & $\begin{array}{l}\text { ADVLEAHEAEAEEPEAGKSEAEDDEDEVD } \\
\text { DLPSSR }\end{array}$ & Phospho(ST) \\
\hline Q9BQ67 & MHNLHGTKPPPSEGSDEEEEEEDEEDEEER & 2 Phospho(ST) \\
\hline Q6IQ49 & VVNTDHGSPEQLQIPVTDSGR & Phospho(ST) \\
\hline P04049 & SASEPSLHR & Phospho(ST) \\
\hline O94875 & TSPGRVDLPGSSTTLTK & Phospho(ST) \\
\hline P41227 & GLAAEDSGGDSK & Phospho(ST) \\
\hline Q01804 & TPSQIIR & Phospho(ST) \\
\hline P78317 & RLPQDHADSCVVSSDDEELSR & 2 Phospho(ST) \\
\hline P31629 & RDLSPR & Phospho(ST) \\
\hline Q6W2J9 & DGSSPPLLEK & Phospho(ST) \\
\hline O60292 & DLRSPR & Phospho(ST) \\
\hline O60292 & ASLRDLRSPR & 2 Phospho(ST) \\
\hline Q9NV56 & VLTANSNPSSPSAAK & 2 Phospho(ST) \\
\hline Q9UDY2 & SRSWEDSPER & 2 Phospho(ST) \\
\hline Q96JG6 & SAYQEYDSDSDVPEELKR & Phospho(ST) \\
\hline P15311 & DKYKTLR & Phospho(ST) \\
\hline
\end{tabular}




\begin{tabular}{|c|c|c|}
\hline Protein & Peptide sequence & Variable modification \\
\hline Q8NFG4 & AHSPAEGASVESSSPGPK & Phospho(ST) \\
\hline Q6U841 & SFADIGK & Phospho(ST) \\
\hline Q8IV63 & LSLFSDGDSSESEDTLSSSER & 2 Phospho(ST) \\
\hline P17706 & EDLSPAFDHSPNK & Phospho(ST) \\
\hline Q9HCD5 & DRSPIR & Phospho(ST) \\
\hline Q16181 & ILEQQNSSRTLEK & Phospho(ST) \\
\hline Q9UHY1 & TPTPEPAEVETR & Phospho(ST) \\
\hline Q5T4S7 & HVTLPSSPR & 2 Phospho(ST) \\
\hline O00418 & $\begin{array}{l}\text { AGHDGDSDGDSDDEEGYFICPITDDPSSNQ } \\
\text { NVNSK }\end{array}$ & 2 Phospho(ST) \\
\hline O15439 & DNEESEQPPVPGTPTLR & Phospho(ST) \\
\hline Q92538 & VPTASYDLDLFTMTWGPTIAALSYVFDK & 3 Phospho(ST) \\
\hline Q8WU90 & AENGERSDLEEDNER & Phospho(ST) \\
\hline O75167 & ASIANSDGPTAGSQTPPFK & Phospho(ST) \\
\hline P31350 & VPLAPITDPQQLQLSPLK & Phospho(ST) \\
\hline Q96PU4 & RPISDDDCPSASK & Phospho(ST) \\
\hline Q86TB9 & RSTSPIIGSPPVR & 2 Phospho(ST) \\
\hline Q56P03 & YYDDIYFDSDSEDEDR & 2 Phospho(ST) \\
\hline Q9H307 & DTSGLER & Phospho(ST) \\
\hline P51812 & NQSPVLEPVGR & Phospho(ST) \\
\hline Q8N488 & DKEISPSVTK & Phospho(ST) \\
\hline Q6IAA8 & LLLDPSSPPTK & Phospho(ST) \\
\hline Q9NQG5 & $\begin{array}{l}\text { TFQQIQEEEDDDYPGSYSPQDPSAGPLLTEE } \\
\text { LIK }\end{array}$ & Phospho(Y) \\
\hline Q9BRD0 & HDTPDPSPR & 2 Phospho(ST) \\
\hline P46937 & ISQSAPVK & Phospho(ST) \\
\hline P46937 & GDSETDLEALFNAVMNPK & Phospho(ST) \\
\hline Q9NPG3 & DRICSDEEEDEEK & Phospho(ST) \\
\hline Q13144 & GGSPQMDDIK & Phospho(ST) \\
\hline Q14980 & SQAPLESSLDSLGDVFLDSGRK & 2 Phospho(ST) \\
\hline Q99700 & RGPEVTSQGVQTSSPACK & Phospho(ST) \\
\hline Q9NYF3 & SLSVPVDLSR & Phospho(ST) \\
\hline Q9NYF3 & RFSLSPSLGPQASR & 2 Phospho(ST) \\
\hline Q5T8P6 & LNHSPPQSSSR & Phospho(ST) \\
\hline Q9NYV6 & MAAPLLHTRLPGDAAASSSAVKK & 2 Phospho(ST) \\
\hline Q9UPT8 & TGSGSPFAGNSPAR & 2 Phospho(ST) \\
\hline P38159 & DVYLSPR & Phospho(ST) \\
\hline P38159 & DYGHSSSR & Phospho(ST) \\
\hline Q96E39 & DVYLSPR & Phospho(ST) \\
\hline Q96E39 & DYGHSSSR & Phospho(ST) \\
\hline Q9NV70 & LTGSTSSLNK & 2 Phospho(ST) \\
\hline Q96F86 & HPNQATPK & Phospho(ST) \\
\hline Q9Y580 & SFSSPENFQR & Phospho(ST) \\
\hline
\end{tabular}




\begin{tabular}{|c|c|c|}
\hline Protein & Peptide sequence & Variable modification \\
\hline Q9BUR4 & VFPEPTESGDEGEELGLPLLSTR & Phospho(ST) \\
\hline Q8TAP8 & APVPEPGLDLSLSPRPDSPQPR & Phospho(ST) \\
\hline Q13286 & RFSDSEGEETVPEPR & Phospho(ST) \\
\hline Q07157 & SREDLSAQPVQTK & Phospho(ST) \\
\hline Q07955 & VKVDGPRSPSYGR & Phospho(ST) \\
\hline Q9BRS2 & VVPGQFDDADSSDSENR & Phospho(ST) \\
\hline Q8IWX8 & SRSPTPPSSAGLGSNSAPPIPDSR & 3 Phospho(ST) \\
\hline P82094 & SVSEINSDDELSGK & Phospho(ST) \\
\hline A0FGR8 & QRGAGAGLSRPGSAR & Phospho(ST) \\
\hline Q92785 & ILEPDDFLDDLDDEDYEEDTPK & Phospho(ST) \\
\hline O95677 & NNPSPPPDSDLER & Phospho(ST) \\
\hline Q9UKJ3 & KGSSPKPK & Phospho(ST) \\
\hline Q9UKJ3 & RSSRSSHR & Phospho(ST) \\
\hline Q9UKJ3 & GPKPEPPGSGSPAPPR & Phospho(ST) \\
\hline O75116 & $\begin{array}{l}\text { SQLQALHIGLDSSSIGSGPGDAEADDGFPES } \\
\mathrm{R}\end{array}$ & 2 Phospho(ST) \\
\hline P51787 & AAASSPPR & Phospho(ST) \\
\hline Q9UMS6 & AQSPTPSLPASWK & 2 Phospho(ST) \\
\hline Q12834 & VQTTPSKPGGDR & Phospho(ST) \\
\hline Q86UU1 & VLTTSPSR & Phospho(ST) \\
\hline Q86UU1 & TRSPSPTLGESLAPHK & 2 Phospho(ST) \\
\hline Q92613 & APYQENDGYCPDLELSDSEAESDGNKEK & 2 Phospho(ST) \\
\hline Q14135 & TGPPPISPSKR & Phospho(ST) \\
\hline Q92974 & QELGSPEER & Phospho(ST) \\
\hline Q92974 & LQDSSDPDTGSEEEGSSRLSPPHSPR & Phospho(ST) \\
\hline Q9BYW2 & RQEIESDSESDGELQDR & 3 Phospho(ST) \\
\hline Q9UHB7 & SSSPGKPQAVSSLNSSHSR & Phospho(ST) \\
\hline P43243 & RDSFDDRGPSLNPVLDYDHGSR & Phospho(ST) \\
\hline P25054 & SELSPVAR & Phospho(ST) \\
\hline P25054 & SKTPPPPPQTAQTK & Phospho(ST) \\
\hline Q9H4A3 & DRPVSQPSLVGSK & Phospho(ST) \\
\hline Q86YD1 & SRSWPASPR & Phospho(ST) \\
\hline Q14684 & KPLTTTPR & Phospho(ST) \\
\hline O95671 & HDSIPAADTFEDLSDVEGGGSEPTQR & Phospho(ST) \\
\hline P41743 & EGLRPGDTTSTFCGTPNYIAPEILR & Phospho(ST) \\
\hline Q16629 & YFQSPSR & 2 Phospho(ST) \\
\hline Q9HBL0 & VATTPGSPSLGR & Phospho(ST) \\
\hline P17480 & GKLPESPK & Phospho(ST) \\
\hline Q08AD1 & SISNEGLTLNNSHVSK & Phospho(ST) \\
\hline Q08AD1 & SSSMSYVDGFIGTWPK & Phospho(ST) \\
\hline Q9Y2K6 & AVPIAVADEGESESEDDDLKPR & 2 Phospho(ST) \\
\hline Q14161 & $\begin{array}{l}\text { TINNQHSVESQDNDQPDYDSVASDEDTDLE } \\
\text { TTASK }\end{array}$ & 2 Phospho(ST) \\
\hline
\end{tabular}




\begin{tabular}{|c|c|c|}
\hline Protein & Peptide sequence & Variable modification \\
\hline P83916 & KADSDSEDKGEESKPK & Phospho(ST) \\
\hline Q8N4C8 & GTPKPPGPPAQPPGPPNASSNPDLR & Phospho(ST) \\
\hline Q9H4L7 & KLSSSSEPYEEDEFNDDQSIK & 3 Phospho(ST) \\
\hline O15015 & TETTMSPPR & Phospho(ST) \\
\hline Q02790 & SNTAGSQSQVETEA & Phospho(ST) \\
\hline Q8NHZ8 & EDVEVVGGSDGEGAIGLSSDPK & Phospho(ST) \\
\hline Q52LW3 & LLLASPPNER & Phospho(ST) \\
\hline Q15527 & KDLGSTEDGDGTDDFLTDKEDEK & Phospho(ST) \\
\hline P05455 & SPSKPLPEVTDEYK & Phospho(ST) \\
\hline Q9NVD7 & SPSVPKSPTPKSPPSR & 2 Phospho(ST) \\
\hline Q15417 & DYQYSDQGIDY & Phospho(ST) \\
\hline Q9UHD8 & SFEVEEVETPNSTPPR & 2 Phospho(ST) \\
\hline Q96S55 & AKGPSPPGAK & Phospho(ST) \\
\hline Q96S55 & RLSESSALK & Phospho(ST) \\
\hline Q7L7X3 & TASLVTR & Phospho(ST) \\
\hline Q96AY2 & LLSSESEDEEEFIPLAQR & 3 Phospho(ST) \\
\hline P36578 & ILKSPEIQR & Phospho(ST) \\
\hline Q5VWQ8 & GDSPELKPR & Phospho(ST) \\
\hline Q6ZRS2 & NRSPADAGR & Phospho(ST) \\
\hline Q92733 & IAAPELHKGDSDSEEDEPTK & 2 Phospho(ST) \\
\hline Q8IWS0 & SSPSDTRPK & Phospho(ST) \\
\hline Q8N5F7 & KESSDSSSKESQEEFLENPWK & 6 Phospho(ST) \\
\hline O15085 & TRNSGIWESPELDR & Phospho(ST) \\
\hline Q6WCQ1 & AEEQQLPPPLSPPSPSTPNHR & 2 Phospho(ST) \\
\hline Q6WCQ1 & DFTNEAPPAPLPDASASPLSPHR & Phospho(ST) \\
\hline P20908 & GSSGPTGPK & Phospho(ST) \\
\hline Q8N3V7 & AASPAKPSSLDLVPNLPK & Phospho(ST) \\
\hline Q765P7 & ALSSAGPIPIRPPIVPVK & Phospho(ST) \\
\hline P23528 & KSSTPEEVK & Phospho(ST) \\
\hline P23528 & SKMIYASSKDAIK & Phospho(ST) \\
\hline Q13427 & SETPPHWR & 2 Phospho(ST) \\
\hline O15013 & TKSLIAQDHR & Phospho(ST) \\
\hline Q96PC5 & AFLSPPTLLEGPLR & Phospho(ST) \\
\hline O43182 & SASSILCSSGGGPNGIFASPR & 2 Phospho(ST) \\
\hline Q5T5U3 & AQPSSSEDELDNVFFK & Phospho(ST) \\
\hline Q9H5H4 & YEPQSPGYEPR & Phospho(ST) \\
\hline Q5T3I0 & DLESCSDDDNQGSKSPK & 3 Phospho(ST) \\
\hline Q9UFC0 & ACASPSAQVEGSPVAGSDGSQPAVK & Phospho(ST) \\
\hline P43487 & $\begin{array}{l}\text { DTHEDHDTSTENTDESNHDPQFEPIVSLPEQ } \\
\text { EIK }\end{array}$ & 2 Phospho(ST) \\
\hline Q9BXB5 & SSPGSVAASPSGGGGR & Phospho(ST) \\
\hline P11474 & AEPASPDSPKGSSETETEPPVALAPGPAPTR & 3 Phospho(ST) \\
\hline Q92614 & YSHSYLSDSDTEAK & Phospho(ST) \\
\hline
\end{tabular}




\begin{tabular}{|c|c|c|}
\hline Protein & Peptide sequence & Variable modification \\
\hline Q6UUV7 & LTQYHGGSLPNVSQLR & Phospho(ST) \\
\hline Q6UUV7 & LFSLSNPSLSTTNLSGPSR & Phospho(ST) \\
\hline Q15424 & ETINNLDTSSSDFTILQEIEEPSLEPENEK & 2 Phospho(ST) \\
\hline O15226 & SSQCHTGSSPR & Phospho(ST) \\
\hline Q69YN4 & VISHDRDSPPPPPPPPPPPQPQPSLK & Phospho(ST) \\
\hline P39880 & QDESEQSR & Phospho(ST) \\
\hline P61073 & GGHSSVSTESESSSFHSS & Phospho(ST) \\
\hline P06454 & KQKTDEDD & Phospho(ST) \\
\hline Q5F1R6 & $\begin{array}{l}\text { QQLEEEEENFSRPQIDENPLDDNSEEEMED } \\
\text { APK }\end{array}$ & Phospho(ST) \\
\hline Q96P48 & LFPEFDDSDYDEVPEEGPGAPAR & Phospho(Y) \\
\hline P63208 & GKTPEEIR & Phospho(ST) \\
\hline Q9Y6D6 & TNFIEADK & Phospho(ST) \\
\hline Q8IZW8 & ASSPHGLGSPLVASPR & Phospho(ST) \\
\hline Q9Y487 & KDSEEEVSLLGSQDIEEGNHQVEDGCR & Phospho(ST) \\
\hline Q8IZ21 & KDTLAMK & Phospho(ST) \\
\hline O75907 & TGSRPSSHGGGGPAAAEEEVR & Phospho(ST) \\
\hline O14647 & KAQTNDSDSDTESKR & 2 Phospho(ST) \\
\hline Q9P2I0 & EADIDSSDESDIEEDIDQPSAHK & 3 Phospho(ST) \\
\hline Q9NRF8 & LSSSDRYSDASDDSFSEPR & Phospho(ST) \\
\hline Q8IY67 & EALGLGPPAAQLTPPPAPVGLR & Phospho(ST) \\
\hline Q68CZ2 & HPPFSPPEPPLSSPASQHK & Phospho(ST) \\
\hline Q3B726 & HQEVQDQDPVFQGSDSSGYQSDHK & Phospho(Y) \\
\hline Q9H330 & GESAPTLSTSPSPSSPSPTSPSPTLGR & Phospho(ST) \\
\hline Q9P275 & DVVLSTSPK & Phospho(ST) \\
\hline Q9P275 & GSSSSSPEHSASSDSTK & Phospho(ST) \\
\hline Q13371 & NSATCHSEDSDLEID & Phospho(ST) \\
\hline Q13371 & LQYYYSSSEDEDSDHEDKDR & 3 Phospho(ST) \\
\hline O15417 & ALSPGLEESGLGLLAR & Phospho(ST) \\
\hline O15164 & NESEDNKFSDDSDDDFVQPR & 3 Phospho(ST) \\
\hline Q9UKY1 & AESISSDEEVHESVDSDNQQNK & 2 Phospho(ST) \\
\hline Q96N66 & AGGGPTLQCPPPSSPEK & Phospho(ST) \\
\hline Q96QE2 & GSNYHLSDNDASDVE & 2 Phospho(ST) \\
\hline Q9UPU7 & DTSPDKGELVSDEEEDT & Phospho(ST) \\
\hline Q09666 & SNSFSDER & Phospho(ST) \\
\hline Q09666 & ISMQDVDLSLGSPK & Phospho(ST) \\
\hline P06748 & GQESFKK & Phospho(ST) \\
\hline P06748 & SIRDTPAK & Phospho(ST) \\
\hline Q9H1E3 & SGKNSQEDSEDSEDKDVK & Phospho(ST) \\
\hline Q9H1E3 & DDSHSAEDSEDEKEDHK & 3 Phospho(ST) \\
\hline Q9H1E3 & EKTPSPKEEDEEPESPPEK & Phospho(ST) \\
\hline Q9H1E3 & EKTPSPKEEDEEPESPPEK & 3 Phospho(ST) \\
\hline Q9UQ35 & SRSRTPR & Phospho(ST) \\
\hline
\end{tabular}




\begin{tabular}{|c|c|c|}
\hline Protein & Peptide sequence & Variable modification \\
\hline Q9UQ35 & SRTSPVTR & Phospho(ST) \\
\hline Q9UQ35 & SDTSSPEVR & Phospho(ST) \\
\hline Q9UQ35 & SRSPQWR & 2 Phospho(ST) \\
\hline Q9UQ35 & GEFSASPMLK & Phospho(ST) \\
\hline Q9UQ35 & SLSYSPVER & 2 Phospho(ST) \\
\hline Q9UQ35 & DGLPRTPSRR & Phospho(ST) \\
\hline Q9UQ35 & KPIDSLRDSR & Phospho(ST) \\
\hline Q9UQ35 & SSSPVTELASR & 2 Phospho(ST) \\
\hline Q9UQ35 & SSTPPRQSPSR & 2 Phospho(ST) \\
\hline Q9UQ35 & SVSPCSNVESR & 2 Phospho(ST) \\
\hline Q9UQ35 & QSHSGSISPYPK & 2 Phospho(ST) \\
\hline Q9UQ35 & SCFESSPDPELK & 2 Phospho(ST) \\
\hline Q9UQ35 & AQTPPGPSLSGSKSPCPQEK & Phospho(ST) \\
\hline Q9UQ35 & SRSGSSQELDVKPSASPQER & 5 Phospho(ST) \\
\hline P02545 & SGAQASSTPLSPTR & 2 Phospho(ST) \\
\hline P02545 & ASASGSGAQVGGPISSGSSASSVTVTR & Phospho(ST) \\
\hline Q8IYB3 & EARSPQPNK & Phospho(ST) \\
\hline Q8IYB3 & RRTPTPPPR & 2 Phospho(ST) \\
\hline Q8IYB3 & TRHSPTPQQSNR & Phospho(ST) \\
\hline Q8IYB3 & KEKTPELPEPSVK & Phospho(ST) \\
\hline Q8IYB3 & VPKPEPIPEPKEPSPEK & Phospho(ST) \\
\hline P08238 & IEDVGSDEEDDSGKDKK & 2 Phospho(ST) \\
\hline Q58FF8 & IEDVGSDEEDDSGKDKK & 2 Phospho(ST) \\
\hline Q13428 & ASSVSTKESPARK & Phospho(ST) \\
\hline Q13428 & TQPSSGVDSAVGTLPATSPQSTSVQAK & Phospho(ST) \\
\hline O00193 & SASPDDDLGSSNWEAADLGNEER & 2 Phospho(ST) \\
\hline Q9BQE3 & DYEEVGADSADGEDEGEEY & Phospho(ST) \\
\hline P55081 & MEREDSSEEEEEEIDDEEIERR & 2 Phospho(ST) \\
\hline Q12888 & $\begin{array}{l}\text { RSDSPEIPFQAAAGPSDGLDASSPGNSFVGL } \\
\mathrm{R}\end{array}$ & Phospho(ST) \\
\hline Q9H6F5 & ALVEFESNPEETREPGSPPSVQR & Phospho(ST) \\
\hline Q15149 & SSSVGSSSSYPISPAVSR & 3 Phospho(ST) \\
\hline Q15149 & $\begin{array}{l}\text { DTHDQLSEPSEVRSYVDPSTDERLSYTQLL } \\
\mathrm{R}\end{array}$ & 4 Phospho(ST) \\
\hline O14974 & SGSYSYLEER & Phospho(ST) \\
\hline O43719 & LFDDSDER & Phospho(ST) \\
\hline O43719 & LFEDDDSNEK & Phospho(ST) \\
\hline O43719 & VFDDESDEKEDEEYADEK & Phospho(ST) \\
\hline O43719 & ELHENVLDKELEENDSENSEFEDDGSEK & 3 Phospho(ST) \\
\hline Q96ST2 & VVSDADDSDSDAVSDK & 4 Phospho(ST) \\
\hline Q96ST2 & TIASDSEEEAGKELSDK & 2 Phospho(ST) \\
\hline Q96ST2 & GHHVTDSENDEPLNLNASDSESEELHR & 5 Phospho(ST) \\
\hline P07910 & AVVPSKR & Phospho(ST) \\
\hline
\end{tabular}




\begin{tabular}{|c|c|c|}
\hline Protein & Peptide sequence & Variable modification \\
\hline P51858 & GNAEGSSDEEGKLVIDEPAKEK & Phospho(ST) \\
\hline Q9Y2W1 & QAYSPR & Phospho(ST) \\
\hline P42167 & VETSEHFR & Phospho(ST) \\
\hline O60841 & KQSFDDNDSEELEDK & Phospho(ST) \\
\hline O60841 & NKPGPNIESGNEDDDASFK & Phospho(ST) \\
\hline P11388 & VPDEEENEESDNEKETEK & Phospho(ST) \\
\hline Q02880 & VVEAVNSDSDSEFGIPK & 2 Phospho(ST) \\
\hline Q02880 & FDSNEEDSASVFSPSFGLK & Phospho(ST) \\
\hline Q02880 & SEDDSAKFDSNEEDSASVFSPSFGLK & Phospho(ST) \\
\hline Q02880 & KTSFDQDSDVDIFPSDFPTEPPSLPR & 2 Phospho(ST) \\
\hline Q02880 & $\begin{array}{l}\text { VKASPITNDGEDEFVPSDGLDKDEYTFSPG } \\
\mathrm{K}\end{array}$ & 3 Phospho(ST) \\
\hline Q02952 & RGSSSDEEGGPK & Phospho(ST) \\
\hline Q8NE71 & KLSVPTSDEEDEVPAPKPR & 3 Phospho(ST) \\
\hline P08670 & LRSSVPGVR & Phospho(ST) \\
\hline P08670 & ETNLDSLPLVDTHSK & Phospho(ST) \\
\hline Q13442 & SLDSDESEDEEDDYQQK & Phospho(ST) \\
\hline Q9NTI5 & EDILENEDEQNSPPK & Phospho(ST) \\
\hline Q9Y3T9 & EAARSPDKPGGSPSASR & 2 Phospho(ST) \\
\hline Q13459 & SPLEHSSPEK & Phospho(ST) \\
\hline Q8WWI1 & EGFESDTDSEFTFK & Phospho(ST) \\
\hline P52756 & GLVAAYSGDSDNEEELVER & 2 Phospho(ST) \\
\hline Q96JM3 & KPGPPLSPEIRSPAGSPELR & 3 Phospho(ST) \\
\hline O14950 & ATSNVFAMFDQSQIQEFK & 2 Phospho(ST) \\
\hline P46100 & LTVSDGESGEEK & 2 Phospho(ST) \\
\hline P46100 & ATSSSNPSSPAPDWYK & 2 Phospho(ST) \\
\hline O95218 & EESDGEYDEFGR & Phospho(ST) \\
\hline O95218 & YNLDASEEEDSNKK & Phospho(ST) \\
\hline O95218 & ENVEYIEREESDGEYDEFGR & Phospho(Y) \\
\hline Q9BWU0 & KPALPVSPAAR & Phospho(ST) \\
\hline P18583 & RSRTPSR & 2 Phospho(ST) \\
\hline P18583 & SFSISPVR & 3 Phospho(ST) \\
\hline P18583 & RRSFSISPSR & 3 Phospho(ST) \\
\hline P19338 & KVVVSPTK & Phospho(ST) \\
\hline P19338 & $\begin{array}{l}\text { KEDSDEEEDDDSEEDEEDDEDEDEDEDEIE } \\
\text { PAAMK }\end{array}$ & 2 Phospho(ST) \\
\hline P22626 & GGGGNFGPGPGSNFR & Phospho(ST) \\
\hline P22626 & GFGDGYNGYGGGPGGGNFGGSPGYGGGR & Phospho(ST) \\
\hline O00566 & SPVFSDEDSDLDFDISK & 3 Phospho(ST) \\
\hline O00566 & KSPVFSDEDSDLDFDISKLEQQSK & 3 Phospho(ST) \\
\hline O15173 & LLKPGEEPSEYTDEEDTKDHNKQD & Phospho(Y) \\
\hline P16401 & KPAGATPK & Phospho(ST) \\
\hline P16401 & SETAPAETATPAPVEKSPAKK & Phospho(ST) \\
\hline
\end{tabular}




\begin{tabular}{|c|c|c|}
\hline Protein & Peptide sequence & Variable modification \\
\hline P27816 & ETERASPIK & Phospho(ST) \\
\hline P27816 & KCSLPAEEDSVLEK & Phospho(ST) \\
\hline Q99590 & VKDSSPGEK & Phospho(ST) \\
\hline Q99590 & VETVSQPSESPKDTIDK & Phospho(ST) \\
\hline Q9Y4F1 & SSSPAPEFLASSPPDNK & Phospho(ST) \\
\hline P20810 & LSDSLGQR & Phospho(ST) \\
\hline Q9Y2X3 & HIKEEPLSEEEPCTSTAIASPEKK & Phospho(ST) \\
\hline P46013 & LKISLGK & Phospho(ST) \\
\hline P46013 & LDQPGNLPGSNR & Phospho(ST) \\
\hline P46013 & SSPELEDTATSSK & Phospho(ST) \\
\hline Q7Z460 & RQSSGSATNVASTPDNR & 2 Phospho(ST) \\
\hline Q9H7N4 & SPFLKPDER & Phospho(ST) \\
\hline Q9H7N4 & GAEETSWSGEER & Phospho(ST) \\
\hline Q01831 & DEALSDGDDLR & Phospho(ST) \\
\hline O95817 & SSTPLHSPSPIR & Phospho(ST) \\
\hline O60716 & SQSSHSYDDSTLPLIDR & Phospho(ST) \\
\hline Q5T200 & DTKESR & Phospho(ST) \\
\hline Q5T200 & SLSPSHLTEDR & Phospho(ST) \\
\hline Q5T200 & KTSAVSSPLLDQQR & Phospho(ST) \\
\hline P46087 & GTDTQTPAVLSPSKTQATLKPK & Phospho(ST) \\
\hline P46087 & EAAAGIQWSEEETEDEEEEKEVTPESGPPK & 3 Phospho(ST) \\
\hline O75475 & AVDITTPK & Phospho(ST) \\
\hline Q14684 & SILVSPTGPSR & Phospho(ST) \\
\hline Q14684 & TPTSSPASSPLVAK & Phospho(ST) \\
\hline P05783 & STFSTNYR & Phospho(ST) \\
\hline P09651 & NQGGYGGSSSSSSYGSGR & Phospho(ST) \\
\hline P28715 & FDSSLLSSDDETK & Phospho(ST) \\
\hline O60832 & AGLESGAEPGDGDSDTTK & Phospho(ST) \\
\hline Q86WB0 & LPLVPESPR & Phospho(ST) \\
\hline Q86WB0 & SWDSSSPVDRPEPEAASPTTR & 2 Phospho(ST) \\
\hline Q86WB0 & SQDATFSPGSEQAEKSPGPIVSR & 2 Phospho(ST) \\
\hline Q8IU81 & NVAEALGHSPKDPGGGGGPVR & Phospho(ST) \\
\hline Q92597 & TASGSSVTSLDGTR & Phospho(ST) \\
\hline Q92597 & SRTASGSSVTSLDGTR & 5 Phospho(ST) \\
\hline P35659 & SLIVEGK & Phospho(ST) \\
\hline O60524 & NPYLLSEEEDDDVDGDVNVEK & Phospho(ST) \\
\hline O60524 & DELNEELIQEESSEDEGEYEEVR & 2 Phospho(ST) \\
\hline Q15648 & SQTPPGVATPPIPK & Phospho(ST) \\
\hline Q15648 & LSSSDSIGPDVTDILSDIAEEASK & 2 Phospho(ST) \\
\hline Q13185 & SLSDSESDDSK & Phospho(ST) \\
\hline Q9P035 & WLDESDAEMELR & Phospho(ST) \\
\hline P10412 & KATGAATPK & Phospho(ST) \\
\hline P10412 & SETAPAAPAAPAPAEKTPVKK & Phospho(ST) \\
\hline
\end{tabular}




\begin{tabular}{|c|c|c|}
\hline Protein & Peptide sequence & Variable modification \\
\hline Q96E09 & RIDFIPVSPAPSPTR & 2 Phospho(ST) \\
\hline Q96QR8 & RGGGSGGGEESEGEEVDED & 2 Phospho(ST) \\
\hline Q9NX63 & YSGAYGASVSDEELK & Phospho(ST) \\
\hline Q9UDY2 & DNSPPPAFKPEPPK & Phospho(ST) \\
\hline O15027 & GSVSQPSTPSPPKPTGIFQTSANSSFEPVK & 3 Phospho(ST) \\
\hline P08559 & YGMGTSVER & Phospho(ST) \\
\hline P29803 & YHGHSMSDPGVSYR & 2 Phospho(ST) \\
\hline Q8WVC0 & KYVISDEEEEDDD & Phospho(ST) \\
\hline Q8WVC0 & MQNTDDEERPQLSDDER & 2 Phospho(ST) \\
\hline Q12948 & GSPQSAAAELSSGLLASAAASSR & Phospho(ST) \\
\hline Q9GZR7 & $\begin{array}{l}\text { SPGKAEAESDALPDDTVIESEALPSDIAAEA } \\
\mathrm{R}\end{array}$ & 2 Phospho(ST) \\
\hline P49023 & ISASSATR & Phospho(ST) \\
\hline Q9UH62 & SIVWYPPWAR & Phospho(ST) \\
\hline Q9HAU0 & GLNVIGASDQSPLQSPSNLR & Phospho(ST) \\
\hline Q14244 & AAPAQVRPPSPGNIRPVK & Phospho(ST) \\
\hline O75376 & SPSPSVR & Phospho(ST) \\
\hline O75376 & AQLSPGIYDDTSAR & Phospho(ST) \\
\hline O75376 & IWEREPAPLLSAQYETLSDSDD & 2 Phospho(ST) \\
\hline Q6UN15 & DHSPTPSVFNSDEER & Phospho(ST) \\
\hline P51532 & AKPVVSDDDSEEEQEEDRSGSGSEED & 5 Phospho(ST) \\
\hline P51532 & QKIEKEDDSEGEESEEEEEGEEEGSESESR & 3 Phospho(ST) \\
\hline Q15154 & NVRSDISDQEEDEESEGCPVSINLSK & 2 Phospho(ST) \\
\hline Q14160 & $\begin{array}{l}\text { LAEAPSPAPTPSPTPVEDLGPQTSTSPGRLSP } \\
\text { DFAEELR }\end{array}$ & Phospho(ST) \\
\hline Q8WUI4 & AQSSPAAPASLSAPEPASQAR & Phospho(ST) \\
\hline Q13247 & SVSPPPKR & 2 Phospho(ST) \\
\hline Q13247 & ARSVSPPPK & 2 Phospho(ST) \\
\hline Q8NC51 & TDKSSASAPDVDDPEAFPALA & Phospho(ST) \\
\hline P05114 & TEESPASDEAGEK & 2 Phospho(ST) \\
\hline Q9Y4H2 & VASPTSGVK & Phospho(ST) \\
\hline Q9Y4H2 & TYSLTTPAR & Phospho(ST) \\
\hline Q9Y4H2 & TDSLAATPPAAK & Phospho(ST) \\
\hline Q9Y4H2 & HNSASVENVSLR & Phospho(ST) \\
\hline Q9Y4H2 & SKSQSSGSSATHPISVPGAR & Phospho(ST) \\
\hline Q9BTC0 & GGDDHDDTSDSDSDGLTLK & 3 Phospho(ST) \\
\hline O43583 & LTVENSPK & Phospho(ST) \\
\hline Q08499 & QHEVEIPSPTQK & Phospho(ST) \\
\hline Q14978 & KKASSSDSEDSSEEEEEVQGPPAK & 5 Phospho(ST) \\
\hline Q01804 & EESSEDENEVSNILR & 2 Phospho(ST) \\
\hline Q9NR30 & SDAGLESDTAMK & Phospho(ST) \\
\hline Q9NR30 & $\begin{array}{l}\text { NGFPHPEPDCNPSEAASEESNSEIEQEIPVEQ } \\
\mathrm{K}\end{array}$ & 3 Phospho(ST) \\
\hline
\end{tabular}




\begin{tabular}{|c|c|c|}
\hline Protein & Peptide sequence & Variable modification \\
\hline Q8WWQ0 & KVLSDSEDEEK & 2 Phospho(ST) \\
\hline Q9NYL9 & DLDEDELLGNLSETELK & Phospho(ST) \\
\hline Q13523 & DASPINRWSPTR & 2 Phospho(ST) \\
\hline Q8N9T8 & YVDEENSDGETSNHR & Phospho(ST) \\
\hline Q96T23 & STLESEKPGSPEAAETSPPSNIIDHCEK & 2 Phospho(ST) \\
\hline Q08945 & QLSESFK & Phospho(ST) \\
\hline Q08945 & EFVSSDESSSGENK & 4 Phospho(ST) \\
\hline P24928 & YSPTSPTYSPTTPK & Phospho(ST) \\
\hline O15344 & ASVSGPNSPSETR & 2 Phospho(ST) \\
\hline O43432 & SPVPAQIAITVPK & Phospho(ST) \\
\hline Q5VTL8 & SQSIEQESQEK & Phospho(ST) \\
\hline P18887 & AIGSTSKPQESPK & Phospho(ST) \\
\hline Q4G0J3 & SRPTSEGSDIESTEPQK & Phospho(ST) \\
\hline Q7Z333 & GQVIIISDSDDDDDER & 2 Phospho(ST) \\
\hline P08651 & NWTEDMEGGISSPVKK & Phospho(ST) \\
\hline Q9NQZ2 & TSAAACAVTDLSDDSDFDEK & 2 Phospho(ST) \\
\hline Q9NWW5 & HGSVSADEAAR & Phospho(ST) \\
\hline Q9BW71 & RPPTPCSDPER & 2 Phospho(ST) \\
\hline Q86VM9 & DRQSPPPAK & Phospho(ST) \\
\hline Q86VM9 & GPTSSPCEEEGDEGEEDRTSDLR & 2 Phospho(ST) \\
\hline Q8WUX9 & ISDAELEAELEK & Phospho(ST) \\
\hline P29590 & AVSPPHLDGPPSPR & 2 Phospho(ST) \\
\hline Q6SPF0 & RGATPPAPPR & Phospho(ST) \\
\hline P53621 & NLSPGAVESDVR & Phospho(ST) \\
\hline Q9Y2X7 & HGSGADSDYENTQSGDPLLGLEGK & Phospho(ST) \\
\hline Q9NQQ7 & GLGSSPDLELLLR & Phospho(ST) \\
\hline Q53H80 & EQPLFTLR & Phospho(ST) \\
\hline Q8WWM7 & QGSGRESPSLASR & Phospho(ST) \\
\hline Q8WX93 & SLPTPAVLLSPTK & Phospho(ST) \\
\hline Q8WX93 & SRDSGDENEPIQER & 2 Phospho(ST) \\
\hline O43765 & SRTPSASNDDQQE & Phospho(ST) \\
\hline O95429 & SSGNSPTPVSR & Phospho(ST) \\
\hline Q9P206 & FSSVSSPQPR & Phospho(ST) \\
\hline Q9P206 & RSTVLGLPQHVQK & Phospho(ST) \\
\hline Q9P206 & LQLERPVSPETQADLQR & Phospho(ST) \\
\hline Q96T37 & HCAPSPDRSPELSSSR & 2 Phospho(ST) \\
\hline Q9UKV3 & SKSPSPPR & 2 Phospho(ST) \\
\hline Q9UKV3 & RLSQPESAEK & Phospho(ST) \\
\hline Q9UKV3 & AAKLSEGSQPAEEEEDQETPSR & 2 Phospho(ST) \\
\hline Q7Z4S6 & IPEPSPVTR & Phospho(ST) \\
\hline Q7Z4S6 & KIPEPSPVTR & Phospho(ST) \\
\hline Q7Z4S6 & EKELSPPPGLPSK & Phospho(ST) \\
\hline P23528 & ASGVAVSDGVIK & Phospho(ST) \\
\hline
\end{tabular}




\begin{tabular}{|c|c|c|}
\hline Protein & Peptide sequence & Variable modification \\
\hline O95297 & SESVVYADIR & Phospho(Y) \\
\hline Q96AE4 & $\begin{array}{l}\text { ADYSTVPPPSSGSAGGGGGGGGGGGVNDA } \\
\text { FKDALQR }\end{array}$ & 2 Phospho(ST) \\
\hline O14545 & ALPSLNTGSSSPR & Phospho(ST) \\
\hline Q96SB4 & $\begin{array}{l}\text { GSAPHSESDLPEQEEEILGSDDDEQEDPNDY } \\
\mathrm{CK}\end{array}$ & Phospho(ST) \\
\hline Q8N556 & SGTSSPQSPVFR & Phospho(ST) \\
\hline Q86TC9 & $\begin{array}{l}\text { EAEQAASEAAGGDTTPGSSPSSLYYEEPLG } \\
\text { QPPR }\end{array}$ & 2 Phospho(ST) \\
\hline P12694 & IGHHSTSDDSSAYR & Phospho(ST) \\
\hline Q4LE39 & DIEVLSEDTDYEEDEVTK & 2 Phospho(ST) \\
\hline Q12894 & SSAQADSGSSDDEAASEAR & 3 Phospho(ST) \\
\hline Q9BX95 & NSLTGEEGQLAR & Phospho(ST) \\
\hline Q14157 & STSAPQMSPGSSDNQSSSPQPAQQK & Phospho(ST) \\
\hline O75909 & KPSPQPSSPR & 2 Phospho(ST) \\
\hline O75396 & NLGSINTELQDVQR & Phospho(ST) \\
\hline P48681 & TSLSFQDPK & Phospho(ST) \\
\hline P48681 & $\begin{array}{l}\text { DGESDGFADEEESGEEGEEDQEEGREPGAG } \\
\mathrm{R}\end{array}$ & 2 Phospho(ST) \\
\hline P29375 & VVSSVSSSPR & Phospho(ST) \\
\hline Q9NQW6 & QPLSEASNQQPLSGGEEK & Phospho(ST) \\
\hline Q12996 & RPNEDSDEDEEK & Phospho(ST) \\
\hline P30419 & GGLSPANDTGAK & Phospho(ST) \\
\hline Q9C0B5 & SSSLKSAQGTGFELGQLQSIR & 2 Phospho(ST) \\
\hline Q15287 & APSPTKR & Phospho(ST) \\
\hline P54259 & SEEISESESEETNAPK & 2 Phospho(ST) \\
\hline Q9NYJ8 & AIGNNSATSPR & Phospho(ST) \\
\hline Q9Y383 & SEDRRSSEER & 2 Phospho(ST) \\
\hline Q8NFH5 & TLGTPTQPGSTPR & Phospho(ST) \\
\hline P53999 & EQISDIDDAVR & Phospho(ST) \\
\hline O14737 & KVMDSDEDDDY & Phospho(ST) \\
\hline Q9NPI6 & ASSPSPLTIGTPESQR & Phospho(ST) \\
\hline Q9NQS7 & IAQVSPGPR & Phospho(ST) \\
\hline Q9NQS7 & DSPAFPDSPWR & Phospho(ST) \\
\hline Q9NQS7 & TLSPTPASATAPTSQGIPTSDEESTPKK & 3 Phospho(ST) \\
\hline O00712 & KPEKPLFSSASPQDSSPR & Phospho(ST) \\
\hline Q5T4S7 & HASTSSPADK & Phospho(ST) \\
\hline Q9Y520 & SESSDFEVVPK & 2 Phospho(ST) \\
\hline Q9H6Y2 & TCEERPAEDGSDEEDPDSMEAPTR & Phospho(ST) \\
\hline Q86YP4 & RPPSPDVIVLSDNEQPSSPR & Phospho(ST) \\
\hline Q9NYL2 & VSQSALNPHQSPDFK & Phospho(ST) \\
\hline Q969E4 & GTDDSPKDSQEDLQER & Phospho(ST) \\
\hline Q969E4 & NEGNLENEGKPEDEVEPDDEGKSDEEEKPD & Phospho(ST) \\
\hline
\end{tabular}




\begin{tabular}{|c|c|c|}
\hline Protein & Peptide sequence & Variable modification \\
\hline & VEGK & \\
\hline Q96QT4 & VSSLAGFTDCHR & Phospho(ST) \\
\hline Q96QT4 & FFVSTPSQPSCK & Phospho(ST) \\
\hline Q71DI3 & KSAPATGGVK & Phospho(ST) \\
\hline Q96EU6 & QLVAGNSPK & Phospho(ST) \\
\hline Q9H019 & IGTNLPLKPCARASFETLPNISDLCLR & 2 Phospho(ST) \\
\hline Q9NYF3 & GSGGGGGPQVPHQSPPKR & Phospho(ST) \\
\hline Q9BQG0 & ESRDPAQPMSPGEATQSGARPADR & Phospho(ST) \\
\hline Q9Y6D5 & GQSQLSNPTDDSWK & 2 Phospho(ST) \\
\hline Q9Y6D5 & ELEKPIQSKPQSPVIQAAAVSPK & 2 Phospho(ST) \\
\hline Q8WW12 & NIGRDTPTSAGPNSFNK & Phospho(ST) \\
\hline Q6U841 & ENSTVDFSK & Phospho(ST) \\
\hline Q9BVJ6 & DSGSQEVLSELR & Phospho(ST) \\
\hline O94875 & SFTSSSPSSPSR & 2 Phospho(ST) \\
\hline Q8TEK3 & ARSTPSPVLQPR & 2 Phospho(ST) \\
\hline Q9Y606 & VPSPLEGSEGDGDTD & Phospho(ST) \\
\hline Q01664 & FIQELSGSSPK & Phospho(ST) \\
\hline Q8WUF5 & SLGSAGPSGTLPR & Phospho(ST) \\
\hline P49450 & RRSPSPTPTPGPSR & 2 Phospho(ST) \\
\hline P40222 & RPEGPGAQAPSSPR & Phospho(ST) \\
\hline Q9HD67 & FDTDDELSYR & Phospho(ST) \\
\hline Q9NZN4 & GPDEAMEDGEEGSDDEAEWVVTK & Phospho(ST) \\
\hline Q9UEG4 & GAESENEEESPR & Phospho(ST) \\
\hline Q9H8V3 & ANTPELK & Phospho(ST) \\
\hline Q8IWA0 & EIPEDVDMEEEKESEDSDEENDFTEK & 2 Phospho(ST) \\
\hline Q13393 & SLQTGVGELHGETR & Phospho(ST) \\
\hline Q8IWS0 & DRSPHR & Phospho(ST) \\
\hline Q96AG3 & SFSTGSDLGHWVTTPPDIPGSR & Phospho(ST) \\
\hline Q8IXM2 & VYEDSGIPLPAESPK & Phospho(ST) \\
\hline Q5TAQ9 & ANRDQDSSDDER & Phospho(ST) \\
\hline P33527 & QLSSSSSYSGDISR & Phospho(ST) \\
\hline P78332 & KEESPPPPK & Phospho(ST) \\
\hline P78332 & LQSFDSPER & Phospho(ST) \\
\hline Q9P2G1 & GVAPADSPEAPR & Phospho(ST) \\
\hline Q86UP2 & QPTPPSEAAASK & Phospho(ST) \\
\hline P51531 & IAKEEESEDESNEEEEEEDEEESESEAK & 3 Phospho(ST) \\
\hline P18669 & FSGWYDADLSPAGHEEAK & Phospho(ST) \\
\hline Q86YS7 & QSSSSDTDLSLTPK & Phospho(ST) \\
\hline Q53EP0 & LNSPPSSIYK & Phospho(ST) \\
\hline P49454 & GSPLLGPVVPGPSPIPSVTEK & 2 Phospho(ST) \\
\hline Q7Z5K2 & YFGFDDLSESEDDEDDDCQVER & Phospho(ST) \\
\hline Q3KQU3 & QSLPASPR & Phospho(ST) \\
\hline Q3KQU3 & RPEASPVQK & Phospho(ST) \\
\hline
\end{tabular}




\begin{tabular}{|c|c|c|}
\hline Protein & Peptide sequence & Variable modification \\
\hline P23588 & SQSSDTEQQSPTSGGGK & Phospho(ST) \\
\hline Q96CP6 & GHVTPNLSR & 2 Phospho(ST) \\
\hline Q96CP6 & EVGDVIALSDITSSGAADRSQEPSPVGSR & 2 Phospho(ST) \\
\hline O43768 & KSSLVTSK & Phospho(ST) \\
\hline Q9UNF1 & ATEVSKTPEAR & Phospho(ST) \\
\hline O43847 & GSLSNAGDPEIVK & Phospho(ST) \\
\hline O43847 & RGSLSNAGDPEIVK & Phospho(ST) \\
\hline Q9ULM3 & FLESPSR & Phospho(ST) \\
\hline P10636 & VAVVRTPPKSPSSAK & 2 Phospho(ST) \\
\hline Q6ZU35 & HSLDGSK & Phospho(ST) \\
\hline Q6ZU35 & HSSTGDSADAGPPAAGSAR & Phospho(ST) \\
\hline Q7RTP6 & LGSPLAVDEALR & Phospho(ST) \\
\hline Q5T3I0 & DLESCSDDDNQGSK & 2 Phospho(ST) \\
\hline Q9NYZ3 & LVDVSPDR & Phospho(ST) \\
\hline Q9H6Z4 & VLSPPKLNEVSSDANR & Phospho(ST) \\
\hline Q9H6Z4 & SAGGSSPEGGEDSDREDGNYCPPVK & 3 Phospho(ST) \\
\hline Q9H6Z4 & SAGGSSPEGGEDSDREDGNYCPPVKR & 3 Phospho(ST) \\
\hline Q92733 & IAAPELHKGDSDSEEDEPTKK & 2 Phospho(ST) \\
\hline P18054 & RSTSLPSR & Phospho(ST) \\
\hline P56524 & AQSSPASATFPVSVQEPPTKPR & Phospho(ST) \\
\hline Q9Y4B4 & LATPPAAQESSR & Phospho(ST) \\
\hline Q7Z2E3 & SGNSDSIER & Phospho(ST) \\
\hline Q5UIP0 & VEEPSQCLASGTAISELIIEDNNASPQK & Phospho(ST) \\
\hline Q6IQ22 & AGGGGGLGAGSPALSGGQGR & Phospho(ST) \\
\hline Q8WU03 & MGYTVPK & Phospho(ST) \\
\hline Q9NVI1 & TIETSPSLSR & Phospho(ST) \\
\hline Q9P2D3 & QWTALIR & Phospho(ST) \\
\hline Q9P2D3 & VSDSPSHIATK & Phospho(ST) \\
\hline Q03188 & SWITIPR & Phospho(ST) \\
\hline Q8N9B5 & GAASPVLQEDHCDSLPSVLQVEEK & Phospho(ST) \\
\hline P36507 & LNQPGTPTR & Phospho(ST) \\
\hline Q3B7J2 & AATSVDAFR & Phospho(ST) \\
\hline Q7Z6Z7 & DGGSGNSTIIVSR & 2 Phospho(ST) \\
\hline Q7Z6Z7 & $\begin{array}{l}\text { REESPMDVDQPSPSAQDTQSIASDGTPQGE } \\
\mathrm{K}\end{array}$ & Phospho(ST) \\
\hline P42684 & TVSTSSQPEENVDR & 2 Phospho(ST) \\
\hline Q86VR2 & $\begin{array}{l}\text { SPSSDLDTDAEGDDFELLDQSELSQLDPASS } \\
\mathrm{R}\end{array}$ & Phospho(ST) \\
\hline Q9UKZ1 & MPGGGASAASGR & Phospho(ST) \\
\hline Q9NP56 & LLSFQR & Phospho(ST) \\
\hline Q9HB75 & VIFPPGATEEPR & Phospho(ST) \\
\hline Q9H307 & SSSSSSSSTSGSSSRDSSSSTSSSSESR & 13 Phospho(ST) \\
\hline A0MZ66 & SMPVLGSVSSVTK & Phospho(ST) \\
\hline
\end{tabular}




\begin{tabular}{|c|c|c|}
\hline Protein & Peptide sequence & Variable modification \\
\hline Q9NZ53 & RDPEDSDVFEEDTHL & Phospho(ST) \\
\hline O14929 & LISPYK & Phospho(ST) \\
\hline Q5VZ03 & YNVTAIPK & Phospho(ST) \\
\hline Q9HCH0 & SPSPGGPQLSPQLPR & Phospho(ST) \\
\hline Q9NR19 & SWSPPPEVSR & Phospho(ST) \\
\hline Q9NR19 & KIAQNDHDLGDMSTVADPSVISHLFSHR & 2 Phospho(ST) \\
\hline P53355 & LFGHSGSGK & Phospho(ST) \\
\hline Q7Z422 & ILGSASPEEEQEKPILDRPTR & Phospho(ST) \\
\hline Q04656 & VSITSEVESTSNSPSSSSLQK & Phospho(ST) \\
\hline Q6WCQ1 & TKDQPDGSSLSPAQSPSQSQPPAASSLR & Phospho(ST) \\
\hline Q6NZY4 & SSSHSSPGSPK & Phospho(ST) \\
\hline Q2NKX8 & NDISPPGR & Phospho(ST) \\
\hline A2RU67 & SPDLGEYDPLTQADSDESEDDLVLNLQK & 2 Phospho(ST) \\
\hline Q969R8 & DTGSPPASEGPTDGSR & Phospho(ST) \\
\hline Q9C0E4 & EGSTLGLTISGGTDKDGKPR & 3 Phospho(ST) \\
\hline Q9ULE0 & SDSDSSTLPR & Phospho(ST) \\
\hline O75976 & SLLSHEFQDETDTEEETLYSSK & 2 Phospho(ST) \\
\hline Q6ZW76 & DVTSPINER & Phospho(ST) \\
\hline Q05193 & RSPTSSPTPQR & 2 Phospho(ST) \\
\hline Q14527 & TAVQYIESSDSEEIETSELPQK & 3 Phospho(ST) \\
\hline Q5SSJ5 & KEPDDSRDEDEDEDESSEEDSEDEEPPPK & 3 Phospho(ST) \\
\hline Q8N3F8 & KPSPAASPATK & 2 Phospho(ST) \\
\hline O75940 & SIFASPESVTGK & Phospho(ST) \\
\hline Q9H501 & FKIDSNISPK & Phospho(ST) \\
\hline P85037 & EEAPASPLRPLYPQISPLK & Phospho(ST) \\
\hline P20020 & IEDSEPHIPLIDDTDAEDDAPTKR & Phospho(ST) \\
\hline Q8N5B7 & VSKDDRSDVESSSEEEDVTTCTK & 4 Phospho(ST) \\
\hline O75533 & AKGSETPGATPGSK & 2 Phospho(ST) \\
\hline Q6GQQ9 & STPESGDSDKESVGSSSTSNEGGR & 2 Phospho(ST) \\
\hline O43663 & TPRTGRHGANK & Phospho(ST) \\
\hline Q9UJF2 & QNSTGQAQIR & Phospho(ST) \\
\hline Q53GS9 & SKRESR & Phospho(ST) \\
\hline O94888 & SESLIDASEDSQLEAAIR & Phospho(ST) \\
\hline Q14568 & ESKDKPEIEDVGSDEEEEK & Phospho(ST) \\
\hline Q8NE01 & ESGSEAER & Phospho(ST) \\
\hline Q92900 & AYQHGGVTGLSQY & Phospho(ST) \\
\hline Q9BXB5 & HLSVGAPGVVTITHHKSPAAAR & Phospho(ST) \\
\hline Q96QE2 & GSNYHLSDNDASDVE & Phospho(ST) \\
\hline Q14980 & EVAEKQEATR & Phospho(ST) \\
\hline Q9UKT5 & SGTNSPPPPFSDWGR & Phospho(ST) \\
\hline Q8TEV9 & SDSQASLTVPLSPQVVR & 3 Phospho(ST) \\
\hline Q8N9E0 & SSQSSTHESESESK & Phospho(ST) \\
\hline $\mathrm{O} 15042$ & RHSTSPSPSRSSSGR & 4 Phospho(ST) \\
\hline
\end{tabular}




\begin{tabular}{lll}
\hline Protein & Peptide sequence & Variable modification \\
\hline P78344 & TQTPPLGQTPQLGLK & Phospho(ST) \\
Q69YQ0 & TSSASRPASLPR & 2 Phospho(ST) \\
Q96GV9 & SLDYLNLDK & Phospho(ST) \\
Q96GV9 & STLTTLPFPGAEANSPGK & Phospho(ST) \\
Q9UNZ2 & KKSPNELVDDLFK & Phospho(ST) \\
Q9BVC5 & KSPSGPVKSPPLSPVGTTPVK & Phospho(ST) \\
O43474 & TTPTLGLEEVLSSR & Phospho(ST) \\
\hline
\end{tabular}


Table S4 Enriched phosphopeptides from the tryptic digest of Hela cell proteins with commercial SPE-Ti-IMAC material as the IMAC sorbent by cLC-MS/MS.

\begin{tabular}{|c|c|c|}
\hline Protein & Peptide sequence & Variable modification \\
\hline P06748 & MQASIEK & Phospho(ST) \\
\hline P06748 & DELHIVEAEAMNYEGSPIK & Phospho(ST) \\
\hline P06748 & DELHIVEAEAMNYEGSPIK & $\begin{array}{l}\text { Oxidation(M); } \\
\text { Phospho(ST) }\end{array}$ \\
\hline P06748 & $\begin{array}{l}\text { CGSGPVHISGQHLVAVEEDAESEDEEEEDV } \\
\mathrm{K}\end{array}$ & Phospho(ST) \\
\hline P06748 & $\begin{array}{l}\text { TVSLGAGAKDELHIVEAEAMNYEGSPIKVT } \\
\text { LATLK }\end{array}$ & Phospho(ST) \\
\hline Q09666 & ADIDVSGPK & Phospho(ST) \\
\hline Q09666 & SNSFSDER & Phospho(ST) \\
\hline Q09666 & FGTFGGLGSK & Phospho(ST) \\
\hline Q09666 & MPFLSISSPK & Phospho(ST) \\
\hline Q09666 & VKTPEMIIQKPK & Phospho(ST) \\
\hline Q09666 & ISAPNVDFNLEGPK & Phospho(ST) \\
\hline Q09666 & VSMPDVELNLKSPK & Phospho(ST) \\
\hline Q09666 & SPQISMSDIDLNLK & Phospho(ST) \\
\hline Q09666 & LPSGSGAASPTGSAVDIR & Phospho(ST) \\
\hline Q09666 & ASLGSLEGEAEAEASSPK & Phospho(ST) \\
\hline Q09666 & ASLGSLEGEAEAEASSPK & 2 Phospho(ST) \\
\hline Q09666 & LKSEDGVEGDLGETQSR & Phospho(ST) \\
\hline Q09666 & VDINTPDVDVHGPDWHLK & Phospho(ST) \\
\hline Q09666 & SSKASLGSLEGEAEAEASSPK & 2 Phospho(ST) \\
\hline Q09666 & EFSGPSTPTGTLEFEGGEVSLEGGK & Phospho(ST) \\
\hline P29401 & ILATPPQEDAPSVDIANIR & Phospho(ST) \\
\hline P05387 & KEESEESDDDMGFGLFD & Phospho(ST) \\
\hline P05387 & KEESEESDDDMGFGLFD & $\begin{array}{r}\text { Oxidation(M); } \\
\text { Phospho(ST) }\end{array}$ \\
\hline P05387 & KEESEESDDDMGFGLFD & 2 Phospho(ST) \\
\hline P05387 & KEESEESDDDMGFGLFD & $\begin{array}{l}\text { Oxidation(M); } \\
2 \text { Phospho(ST) }\end{array}$ \\
\hline P05387 & DEKKEESEESDDDMGFGLFD & 2 Phospho(ST) \\
\hline P05386 & KEESEESDDDMGFGLFD & Phospho(ST) \\
\hline P05386 & KEESEESDDDMGFGLFD & $\begin{array}{l}\text { Oxidation(M); } \\
\text { Phospho(ST) }\end{array}$ \\
\hline P05386 & KEESEESDDDMGFGLFD & 2 Phospho(ST) \\
\hline P05386 & KEESEESDDDMGFGLFD & $\begin{array}{l}\text { Oxidation(M); } \\
2 \text { Phospho(ST) }\end{array}$ \\
\hline P35579 & DELADEIANSSGK & Phospho(ST) \\
\hline P35579 & KGAGDGSDEEVDGK & Phospho(ST) \\
\hline P35579 & GAGDGSDEEVDGKADGAEAKPAE & Phospho(ST) \\
\hline
\end{tabular}




\begin{tabular}{|c|c|c|}
\hline Protein & Peptide sequence & Variable modification \\
\hline P35579 & KGAGDGSDEEVDGKADGAEAKPAE & Phospho(ST) \\
\hline P08238 & EISDDEAEEEK & Phospho(ST) \\
\hline P08238 & IEDVGSDEEDDSGK & Phospho(ST) \\
\hline P08238 & EKEISDDEAEEEK & Phospho(ST) \\
\hline P08238 & IEDVGSDEEDDSGKDK & Phospho(ST) \\
\hline P08238 & IEDVGSDEEDDSGKDK & 2 Phospho(ST) \\
\hline P08238 & EKEISDDEAEEEKGEK & Phospho(ST) \\
\hline P08238 & IEDVGSDEEDDSGKDKK & Phospho(ST) \\
\hline P07900 & DKEVSDDEAEEK & Phospho(ST) \\
\hline P07900 & ERDKEVSDDEAEEK & Phospho(ST) \\
\hline P07900 & ESEDKPEIEDVGSDEEEEKK & Phospho(ST) \\
\hline P07900 & EEKESEDKPEIEDVGSDEEEEKK & Phospho(ST) \\
\hline P07900 & ESEDKPEIEDVGSDEEEEKKDGDK & Phospho(ST) \\
\hline Q58FF8 & IEDVGSDEEDDSGK & Phospho(ST) \\
\hline Q58FF8 & IEDVGSDEEDDSGKDK & Phospho(ST) \\
\hline Q58FF8 & IEDVGSDEEDDSGKDK & 2 Phospho(ST) \\
\hline Q58FF8 & IEDVGSDEEDDSGKDKK & Phospho(ST) \\
\hline Q58FF7 & EISDDEAEEEK & Phospho(ST) \\
\hline P80723 & ETPAATEAPSSTPK & Phospho(ST) \\
\hline P80723 & KAEGAATEEEGTPK & Phospho(ST) \\
\hline P80723 & AEGAATEEEGTPKESEPQAAAEPAEAK & Phospho(ST) \\
\hline P80723 & AQGPAASAEEPKPVEAPAANSDQTVTVKE & Phospho(ST) \\
\hline P61978 & DYDDMSPR & Phospho(ST) \\
\hline \multirow[t]{2}{*}{ P61978 } & DYDDMSPR & Oxidation(M); \\
\hline & & Phospho(ST) \\
\hline P61978 & IILDLISESPIK & Phospho(ST) \\
\hline P61978 & GSYGDLGGPIITTQVTIPK & Phospho(ST) \\
\hline Q8IYB3 & RRSSPVR & Phospho(ST) \\
\hline Q8IYB3 & RSSPVRR & Phospho(ST) \\
\hline Q8IYB3 & YSPPIQR & Phospho(ST) \\
\hline Q8IYB3 & RYSPPIQR & Phospho(ST) \\
\hline Q8IYB3 & RRSPSLSSK & 2 Phospho(ST) \\
\hline Q8IYB3 & RYSPSPPPK & 2 Phospho(ST) \\
\hline Q8IYB3 & KAASPSPQSVR & Phospho(ST) \\
\hline Q8IYB3 & RRTPSPPPR & 2 Phospho(ST) \\
\hline Q8IYB3 & RRTPTPPPR & 2 Phospho(ST) \\
\hline Q8IYB3 & RESPSPAPKPR & Phospho(ST) \\
\hline Q8IYB3 & APQTSSSPPPVR & Phospho(ST) \\
\hline Q8IYB3 & RLSPSASPPR & 3 Phospho(ST) \\
\hline Q8IYB3 & RRSPSPPPTR & 2 Phospho(ST) \\
\hline Q8IYB3 & RRSPSPAPPPR & 2 Phospho(ST) \\
\hline Q8IYB3 & EKTPELPEPSVK & Phospho(ST) \\
\hline Q8IYB3 & HRPSPPATPPPK & 2 Phospho(ST) \\
\hline
\end{tabular}




\begin{tabular}{|c|c|c|}
\hline Protein & Peptide sequence & Variable modification \\
\hline Q8IYB3 & KVELSESEEDK & 2 Phospho(ST) \\
\hline Q8IYB3 & TRHSPTPQQSNR & Phospho(ST) \\
\hline Q8IYB3 & RQSPSPSTRPIR & 2 Phospho(ST) \\
\hline Q8IYB3 & KEKTPELPEPSVK & Phospho(ST) \\
\hline Q8IYB3 & KVELSESEEDKGGK & Phospho(ST) \\
\hline Q8IYB3 & KETESEAEDNLDDLEK & Phospho(ST) \\
\hline Q8IYB3 & VPKPEPIPEPKEPSPEK & Phospho(ST) \\
\hline Q8IYB3 & KETESEAEDNLDDLEK & 2 Phospho(ST) \\
\hline Q8IYB3 & KPPAPPSPVQSQSPSTNWSPAVPVK & Phospho(ST) \\
\hline Q8IYB3 & KPPAPPSPVQSQSPSTNWSPAVPVK & 2 Phospho(ST) \\
\hline Q8IYB3 & KPPAPPSPVQSQSPSTNWSPAVPVKK & 2 Phospho(ST) \\
\hline P02545 & LRLSPSPTSQR & Phospho(ST) \\
\hline P02545 & SGAQASSTPLSPTR & Phospho(ST) \\
\hline P02545 & SGAQASSTPLSPTR & 2 Phospho(ST) \\
\hline P02545 & ASSHSSQTQGGGSVTK & Phospho(ST) \\
\hline Q9H1E3 & ATVTPSPVK & Phospho(ST) \\
\hline Q9H1E3 & ATVTPSPVKGK & 2 Phospho(ST) \\
\hline Q9H1E3 & EEDEEPESPPEK & Phospho(ST) \\
\hline Q9H1E3 & EEDEEPESPPEKK & Phospho(ST) \\
\hline Q9H1E3 & TPSPKEEDEEPESPPEKK & Phospho(ST) \\
\hline Q9H1E3 & VVDYSQFQESDDADEDYGR & Phospho(ST) \\
\hline Q9H1E3 & KDDSHSAEDSEDEKEDHK & 3 Phospho(ST) \\
\hline Q9H1E3 & KVVDYSQFQESDDADEDYGR & Phospho(ST) \\
\hline Q15149 & SDEGQLSPATR & Phospho(ST) \\
\hline Q15149 & SSSVGSSSSYPISPAVSR & Phospho(ST) \\
\hline Q15149 & RTPHVQAVQGPLGSPPK & Phospho(ST) \\
\hline Q15149 & GYYSPYSVSGSGSTAGSR & Phospho(ST) \\
\hline Q15149 & SSSVGSSSSYPISPAVSR & 2 Phospho(ST) \\
\hline Q15149 & ELEEVSPETPVVPATTQR & Phospho(ST) \\
\hline Q15149 & SRSSSVGSSSSYPISPAVSR & 3 Phospho(ST) \\
\hline P58107 & AEAEAGSPRPDPR & Phospho(ST) \\
\hline P58107 & QVSASELHTSGILGPETLR & Phospho(ST) \\
\hline P58107 & RQVSASELHTSGILGPETLR & Phospho(ST) \\
\hline P63104 & TAFDEAIAELDTLSEESYK & Phospho(ST) \\
\hline P68363 & SIQFVDWCPTGFK & Phospho(ST) \\
\hline P68363 & TIGGGDDSFNTFFSETGAGK & Phospho(ST) \\
\hline P68363 & DYEEVGVDSVEGEGEEEGEEY & Phospho(ST) \\
\hline Q9BQE3 & TIGGGDDSFNTFFSETGAGK & Phospho(ST) \\
\hline Q9BQE3 & DYEEVGADSADGEDEGEEY & Phospho(ST) \\
\hline P68366 & SIQFVDWCPTGFK & Phospho(ST) \\
\hline Q71U36 & TIGGGDDSFNTFFSETGAGK & Phospho(ST) \\
\hline Q71U36 & DYEEVGVDSVEGEGEEEGEEY & Phospho(ST) \\
\hline Q13748 & TIGGGDDSFNTFFSETGAGK & Phospho(ST) \\
\hline
\end{tabular}




\begin{tabular}{|c|c|c|}
\hline Protein & Peptide sequence & Variable modification \\
\hline Q9NYF8 & RHSRSPR & 2 Phospho(ST) \\
\hline Q9NYF8 & IDISPSTLR & Phospho(ST) \\
\hline Q9NYF8 & RIDISPSTLR & Phospho(ST) \\
\hline Q9NYF8 & DLFDYSPPLHK & Phospho(ST) \\
\hline Q9NYF8 & KAEGEPQEESPLK & Phospho(ST) \\
\hline Q9NYF8 & LKDLFDYSPPLHK & Phospho(ST) \\
\hline Q9NYF8 & KAEGEPQEESPLKSK & Phospho(ST) \\
\hline Q9NYF8 & YSPSQNSPIHHIPSR & Phospho(ST) \\
\hline Q9NYF8 & FNDSEGDDTEETEDYR & Phospho(ST) \\
\hline Q9NYF8 & FNDSEGDDTEETEDYR & 2 Phospho(ST) \\
\hline Q9NYF8 & AEGEWEDQEALDYFSDK & Phospho(ST) \\
\hline Q9NYF8 & SSATSGDIWPGLSAYDNSPR & Phospho(ST) \\
\hline Q9NYF8 & QKFNDSEGDDTEETEDYR & Phospho(ST) \\
\hline Q9NYF8 & AEGEWEDQEALDYFSDKESGK & Phospho(ST) \\
\hline Q9NYF8 & GRAEGEWEDQEALDYFSDKESGK & Phospho(ST) \\
\hline P51858 & AGDLLEDSPK & Phospho(ST) \\
\hline P51858 & RAGDLLEDSPK & Phospho(ST) \\
\hline P51858 & AGDLLEDSPKRPK & Phospho(ST) \\
\hline P51858 & RAGDLLEDSPKRPK & Phospho(ST) \\
\hline P51858 & GNAEGSSDEEGKLVIDEPAK & 2 Phospho(ST) \\
\hline P51858 & GNAEGSSDEEGKLVIDEPAKEK & 2 Phospho(ST) \\
\hline P24534 & DDDDIDLFGSDDEEESEEAK & Phospho(ST) \\
\hline P24534 & DDDDIDLFGSDDEEESEEAKR & Phospho(ST) \\
\hline P24534 & $\begin{array}{l}\text { YGPADVEDTTGSGATDSKDDDDIDLFGSD } \\
\text { DEEESEEAK }\end{array}$ & Phospho(ST) \\
\hline P24534 & $\begin{array}{l}\text { YGPADVEDTTGSGATDSKDDDDIDLFGSD } \\
\text { DEEESEEAKR }\end{array}$ & Phospho(ST) \\
\hline P29692 & ATAPQTQHVSPMR & Phospho(ST) \\
\hline P29692 & ATAPQTQHVSPMR & $\begin{array}{l}\text { Oxidation(M); } \\
\text { Phospho(ST) }\end{array}$ \\
\hline P29692 & KPATPAEDDEDDDIDLFGSDNEEEDK & Phospho(ST) \\
\hline P29692 & $\begin{array}{l}\text { KPATPAEDDEDDDIDLFGSDNEEEDKEAA } \\
\text { QLR }\end{array}$ & Phospho(ST) \\
\hline P29692 & $\begin{array}{l}\text { KPATPAEDDEDDDIDLFGSDNEEEDKEAA } \\
\text { QLREER }\end{array}$ & Phospho(ST) \\
\hline P29692 & $\begin{array}{l}\text { QVEPPAKKPATPAEDDEDDDIDLFGSDNEE } \\
\text { EDKEAAQLR }\end{array}$ & Phospho(ST) \\
\hline Q13428 & KGAAPTPPGK & Phospho(ST) \\
\hline Q13428 & TVANLLSGKSPR & Phospho(ST) \\
\hline Q13428 & ASSVSTKESPARK & Phospho(ST) \\
\hline Q13428 & LGAGEGGEASVSPEK & Phospho(ST) \\
\hline Q13428 & TSQVGAASAPAKESPR & Phospho(ST) \\
\hline Q13428 & SLGNILQAKPTSSPAK & Phospho(ST) \\
\hline
\end{tabular}




\begin{tabular}{|c|c|c|}
\hline Protein & Peptide sequence & Variable modification \\
\hline Q13428 & SPAGPAATPAQAQAASTPR & Phospho(ST) \\
\hline Q13428 & LGAGEGGEASVSPEKTSTTSK & Phospho(ST) \\
\hline Q13428 & TQPSSGVDSAVGTLPATSPQSTSVQAK & Phospho(ST) \\
\hline Q13428 & AKKPEEESESSEEGSESEEEAPAGTR & 5 Phospho(ST) \\
\hline Q13428 & AEKQEDSESSEEESDSEEAAASPAQVK & 5 Phospho(ST) \\
\hline Q8NE71 & LSVPTSDEEDEVPAPKPR & Phospho(ST) \\
\hline Q8NE71 & KLSVPTSDEEDEVPAPKPR & Phospho(ST) \\
\hline Q8NE71 & QQPPEPEWIGDGESTSPSDK & Phospho(ST) \\
\hline Q8NE71 & KLSVPTSDEEDEVPAPKPR & 3 Phospho(ST) \\
\hline Q8NE71 & AEQGSEEEGEGEEEEEEGGESK & Phospho(ST) \\
\hline Q8NE71 & KAEQGSEEEGEGEEEEEEGGESK & Phospho(ST) \\
\hline P21333 & APSVANVGSHCDLSLK & Phospho(ST) \\
\hline P21333 & RAPSVANVGSHCDLSLK & Phospho(ST) \\
\hline Q14315 & LGSFGSITR & Phospho(ST) \\
\hline P46821 & GAESPFEEK & Phospho(ST) \\
\hline P46821 & SLMSSPEDLTK & $\begin{array}{l}\text { Oxidation(M); } \\
2 \text { Phospho(ST) }\end{array}$ \\
\hline P46821 & VQSLEGEKLSPK & Phospho(ST) \\
\hline P46821 & TTKSPSDSGYSYETIGK & Phospho(ST) \\
\hline P46821 & ESSPLYSPTFSDSTSAVK & Phospho(ST) \\
\hline P46821 & TTRTPEEGGYSYDISEK & Phospho(ST) \\
\hline P46821 & HMDPPPAPVQDRSPSPR & 2 Phospho(ST) \\
\hline P46821 & TTKTPEDGDYSYEIIEK & Phospho(ST) \\
\hline P46821 & ASVSPMDEPVPDSESPIEK & Phospho(ST) \\
\hline P46821 & VLSPLRSPPLIGSESAYESFLSADDK & Phospho(ST) \\
\hline P46821 & VLSPLRSPPLIGSESAYESFLSADDK & 2 Phospho(ST) \\
\hline P46821 & VSAEAEVAPVSPEVTQEVVEEHCASPEDK & Phospho(ST) \\
\hline P46821 & $\begin{array}{l}\text { AEEDMDEAIEKGEAEQSEEEADEEDKAED } \\
\text { AR }\end{array}$ & Phospho(ST) \\
\hline Q15185 & DWEDDSDEDMSNFDR & Phospho(ST) \\
\hline Q15185 & DWEDDSDEDMSNFDR & $\begin{array}{l}\text { Oxidation(M); } \\
\text { Phospho(ST) }\end{array}$ \\
\hline P42166 & SSTPLPTISSSAENTR & Phospho(ST) \\
\hline P42166 & GPPDFSSDEEREPTPVLGSGAAAAGR & Phospho(ST) \\
\hline P42166 & GPPDFSSDEEREPTPVLGSGAAAAGR & 2 Phospho(ST) \\
\hline P42166 & GPPDFSSDEEREPTPVLGSGAAAAGR & 3 Phospho(ST) \\
\hline P42167 & AKTPVTLK & Phospho(ST) \\
\hline P42167 & MEESFSSK & Phospho(ST) \\
\hline P42167 & SSTPLPTISSSAENTR & Phospho(ST) \\
\hline P42167 & HASPILPITEFSDIPR & Phospho(ST) \\
\hline P42167 & GPPDFSSDEEREPTPVLGSGAAAAGR & Phospho(ST) \\
\hline P42167 & GPPDFSSDEEREPTPVLGSGAAAAGR & 2 Phospho(ST) \\
\hline P42167 & GPPDFSSDEEREPTPVLGSGAAAAGR & 3 Phospho(ST) \\
\hline
\end{tabular}




\begin{tabular}{|c|c|c|}
\hline Protein & Peptide sequence & Variable modification \\
\hline Q9UQ35 & SRLSLR & Phospho(ST) \\
\hline Q9UQ35 & VPSPTPAPK & Phospho(ST) \\
\hline Q9UQ35 & ELSNSPLR & Phospho(ST) \\
\hline Q9UQ35 & DTLRTPPR & Phospho(ST) \\
\hline Q9UQ35 & KHRSPTPK & 2 Phospho(ST) \\
\hline Q9UQ35 & ALPQTPRPR & Phospho(ST) \\
\hline Q9UQ35 & GEFSASPMLK & Phospho(ST) \\
\hline Q9UQ35 & TVARTPLGQR & Phospho(ST) \\
\hline Q9UQ35 & SLSYSPVER & 2 Phospho(ST) \\
\hline Q9UQ35 & SSSPVTELASR & Phospho(ST) \\
\hline Q9UQ35 & DGLPRTPSRR & Phospho(ST) \\
\hline Q9UQ35 & RRPSPQPSPR & Phospho(ST) \\
\hline Q9UQ35 & SSSPVTELASR & 2 Phospho(ST) \\
\hline Q9UQ35 & RGRSPSPKPR & 2 Phospho(ST) \\
\hline Q9UQ35 & SRTPPSAPSQSR & Phospho(ST) \\
\hline Q9UQ35 & ENSFGSPLEFR & Phospho(ST) \\
\hline Q9UQ35 & QSHSGSISPYPK & Phospho(ST) \\
\hline Q9UQ35 & VSGRTSPPLLDR & Phospho(ST) \\
\hline Q9UQ35 & QSHSESPSLQSK & Phospho(ST) \\
\hline Q9UQ35 & SRSPSSPELNNK & 2 Phospho(ST) \\
\hline Q9UQ35 & SRSSSPVTELASR & 3 Phospho(ST) \\
\hline Q9UQ35 & CRSPGMLEPLGSSR & Phospho(ST) \\
\hline Q9UQ35 & CRSPGMLEPLGSSR & $\begin{array}{l}\text { Oxidation(M); } \\
\text { Phospho(ST) }\end{array}$ \\
\hline Q9UQ35 & MALPPQEDATASPPR & Phospho(ST) \\
\hline Q9UQ35 & SSGHSSSELSPDAVEK & Phospho(ST) \\
\hline Q9UQ35 & THTTALAGRSPSPASGR & 2 Phospho(ST) \\
\hline Q9UQ35 & SSTPPGESYFGVSSLQLK & Phospho(ST) \\
\hline Q9UQ35 & AGMSSNQSISSPVLDAVPR & Phospho(ST) \\
\hline Q9UQ35 & QGSITSPQANEQSVTPQR & Phospho(ST) \\
\hline Q9UQ35 & AGMSSNQSISSPVLDAVPR & $\begin{array}{l}\text { Oxidation(M); } \\
\text { Phospho(ST) }\end{array}$ \\
\hline Q9UQ35 & QGSITSPQANEQSVTPQR & 2 Phospho(ST) \\
\hline Q9UQ35 & QGSITSPQANEQSVTPQRR & Phospho(ST) \\
\hline Q9UQ35 & AQTPPGPSLSGSKSPCPQEK & 2 Phospho(ST) \\
\hline Q9UQ35 & HASSSPESPKPAPAPGSHR & 4 Phospho(ST) \\
\hline Q9UQ35 & QGSITSPQANEQSVTPQRR & 2 Phospho(ST) \\
\hline Q9UQ35 & AGMSSNQSISSPVLDAVPRTPSR & 2 Phospho(ST) \\
\hline Q9UQ35 & RGEGDAPFSEPGTTSTQRPSSPETATK & Phospho(ST) \\
\hline Q9UQ35 & HGGSPQPLATTPLSQEPVNPPSEASPTR & 3 Phospho(ST) \\
\hline Q9UQ35 & MGQAPSQSLLPPAQDQPRSPVPSAFSDQSR & Phospho(ST) \\
\hline P27824 & AEEDEILNRSPR & Phospho(ST) \\
\hline P27824 & SDAEEDGGTVSQEEEDR & Phospho(ST) \\
\hline
\end{tabular}




\begin{tabular}{|c|c|c|}
\hline Protein & Peptide sequence & Variable modification \\
\hline P27824 & QKSDAEEDGGTVSQEEEDR & Phospho(ST) \\
\hline P27824 & QKSDAEEDGGTVSQEEEDRKPK & Phospho(ST) \\
\hline P27824 & QKSDAEEDGGTVSQEEEDRKPK & 2 Phospho(ST) \\
\hline Q9NTI5 & EDILENEDEQNSPPK & Phospho(ST) \\
\hline Q9NTI5 & METVSNASSSSNPSSPGR & Phospho(ST) \\
\hline \multirow[t]{2}{*}{ Q9NTI5 } & METVSNASSSSNPSSPGR & Oxidation(M); \\
\hline & & Phospho(ST) \\
\hline Q9NTI5 & AESPESSAIESTQSTPQK & Phospho(ST) \\
\hline Q9NTI5 & EDILENEDEQNSPPKK & Phospho(ST) \\
\hline Q9NTI5 & AESPESSAIESTQSTPQK & 2 Phospho(ST) \\
\hline Q9NTI5 & LKEDILENEDEQNSPPK & Phospho(ST) \\
\hline P14618 & LDIDSPPITAR & Phospho(ST) \\
\hline P08670 & LRSSVPGVR & Phospho(ST) \\
\hline P08670 & ETNLDSLPLVDTHSK & Phospho(ST) \\
\hline P29966 & LSGFSFK & Phospho(ST) \\
\hline P29966 & LSGFSFKK & Phospho(ST) \\
\hline P29966 & AEDGATPSPSNETPK & Phospho(ST) \\
\hline P29966 & GEAAAERPGEAAVASSPSK & Phospho(ST) \\
\hline O95218 & EESDGEYDEFGR & Phospho(ST) \\
\hline O95218 & YNLDASEEEDSNKK & Phospho(ST) \\
\hline O95218 & EVEDKESEGEEEDEDEDLSK & Phospho(ST) \\
\hline O95218 & ENVEYIEREESDGEYDEFGRK & Phospho(ST) \\
\hline P49792 & YSLSPSK & Phospho(ST) \\
\hline P49792 & FESPATGILSPR & Phospho(ST) \\
\hline P49792 & ELVGPPLAETVFTPK & Phospho(ST) \\
\hline P49792 & LNQSGTSVGTDEESDVTQEEER & Phospho(ST) \\
\hline P49792 & EDALDDSVSSSSVHASPLASSPVRK & Phospho(ST) \\
\hline \multirow[t]{2}{*}{ P49792 } & VGEDEDGSDEEVVHNEDIHFEPIVSLPEVE & Phospho(ST) \\
\hline & $\mathrm{VK}$ & \\
\hline A6NKT7 & YSLSPSK & Phospho(ST) \\
\hline A6NKT7 & LNQSGTSVGTDEESDVTQEEER & Phospho(ST) \\
\hline A6NKT7 & EDALDDSVSSSSVHASPLASSPVRK & Phospho(ST) \\
\hline P0DJD0 & YSLSPSK & Phospho(ST) \\
\hline P0DJD0 & LNQSGTSVGTDEESDVTQEEER & Phospho(ST) \\
\hline O14715 & YSLSPSK & Phospho(ST) \\
\hline O14715 & EDALDDSVSSSSVHASPLASSPVRK & Phospho(ST) \\
\hline P49736 & GNDPLTSSPGR & Phospho(ST) \\
\hline P49736 & RTDALTSSPGR & Phospho(ST) \\
\hline P49736 & RGNDPLTSSPGR & Phospho(ST) \\
\hline P49736 & GLLYDSDEEDEERPAR & Phospho(ST) \\
\hline P49736 & RGLLYDSDEEDEERPAR & Phospho(ST) \\
\hline O14974 & LASTSDIEEK & Phospho(ST) \\
\hline O14974 & TGSYGALAEITASK & Phospho(ST) \\
\hline
\end{tabular}




\begin{tabular}{|c|c|c|}
\hline Protein & Peptide sequence & Variable modification \\
\hline O14974 & KTGSYGALAEITASK & Phospho(ST) \\
\hline O14974 & RSTQGVTLTDLQEAEK & Phospho(ST) \\
\hline P05787 & VGSSNFR & Phospho(ST) \\
\hline P05787 & ISSSSFSR & Phospho(ST) \\
\hline P05787 & LVSESSDVLPK & Phospho(ST) \\
\hline P05787 & SLDMDSIIAEVK & Phospho(ST) \\
\hline P05787 & SLDMDSIIAEVK & 2 Phospho(ST) \\
\hline P08729 & SLDLDGIIAEVK & Phospho(ST) \\
\hline Q8WWI1 & ISINQTPGK & Phospho(ST) \\
\hline Q8WWI1 & SYTMDDAWK & Phospho(ST) \\
\hline Q8WWI1 & GESLDNLDSPR & Phospho(ST) \\
\hline Q8WWI1 & SHSPSASQSGSQLR & Phospho(ST) \\
\hline Q8WWI1 & MYSFDDVLEEGK & Phospho(ST) \\
\hline Q8WWI1 & SRSTTELDDYSTNK & Phospho(ST) \\
\hline Q8WWI1 & SASVNKEPVSLPGIMR & Phospho(ST) \\
\hline Q8WWI1 & LPSPTSPFSSLSQDQAATSK & Phospho(ST) \\
\hline Q8WWI1 & ATLSSTSGLDLMSESGEGEISPQR & Phospho(ST) \\
\hline P18615 & SVWGSLAVQNSPK & Phospho(ST) \\
\hline P18615 & SLSEQPVMDTATATEQAK & Phospho(ST) \\
\hline Q6PKG0 & SLPTTVPESPNYR & Phospho(ST) \\
\hline Q6PKG0 & ILIVTQTPHYMR & Phospho(ST) \\
\hline Q6PKG0 & SLPTTVPESPNYR & Phospho(ST); Phospho(Y) \\
\hline Q6PKG0 & AVTPVPTKTEEVSNLK & Phospho(ST) \\
\hline Q6PKG0 & GLSASLPDLDSENWIEVK & Phospho(ST) \\
\hline Q6PKG0 & KNTFTAWSDEESDYEIDDR & 2 Phospho(ST) \\
\hline Q6PKG0 & $\begin{array}{l}\text { ESPRPLQLPGAEGPAISDGEEGGGEPGAGG } \\
\text { GAAGAAGAGR }\end{array}$ & Phospho(ST) \\
\hline P07910 & MESEGGADDSAEEGDLLDDDDNEDR & Phospho(ST) \\
\hline P07910 & MESEGGADDSAEEGDLLDDDDNEDR & $\begin{array}{l}\text { Oxidation(M); } \\
\text { Phospho(ST) }\end{array}$ \\
\hline P07910 & MESEGGADDSAEEGDLLDDDDNEDR & 2 Phospho(ST) \\
\hline P07910 & $\begin{array}{l}\text { MESEGGADDSAEEGDLLDDDDNEDRGDD } \\
\text { QLELIK }\end{array}$ & Phospho(ST) \\
\hline P07910 & $\begin{array}{l}\text { MESEGGADDSAEEGDLLDDDDNEDRGDD } \\
\text { QLELIK }\end{array}$ & $\begin{array}{l}\text { Oxidation(M); } \\
\text { Phospho(ST) }\end{array}$ \\
\hline P07910 & $\begin{array}{l}\text { MESEGGADDSAEEGDLLDDDDNEDRGDD } \\
\text { QLELIKDDEK }\end{array}$ & Phospho(ST) \\
\hline P07910 & $\begin{array}{l}\text { MESEGGADDSAEEGDLLDDDDNEDRGDD } \\
\text { QLELIKDDEK }\end{array}$ & $\begin{array}{l}\text { Oxidation(M); } \\
\text { Phospho(ST) }\end{array}$ \\
\hline P07910 & $\begin{array}{l}\text { MESEGGADDSAEEGDLLDDDDNEDRGDD } \\
\text { QLELIKDDEK }\end{array}$ & 2 Phospho(ST) \\
\hline Q9H6F5 & AGLGSPERPPK & Phospho(ST) \\
\hline Q9H6F5 & LGGLRPESPESLTSVSR & Phospho(ST) \\
\hline
\end{tabular}




\begin{tabular}{|c|c|c|}
\hline Protein & Peptide sequence & Variable modification \\
\hline Q9H6F5 & AGLGSPERPPKTSPGSPR & Phospho(ST) \\
\hline Q9H6F5 & LGGLRPESPESLTSVSR & 2 Phospho(ST) \\
\hline Q9H6F5 & AGLGSPERPPKTSPGSPR & 2 Phospho(ST) \\
\hline Q9H6F5 & LQQGAGLESPQGQPEPGAASPQR & Phospho(ST) \\
\hline Q9H6F5 & LQQGAGLESPQGQPEPGAASPQR & 2 Phospho(ST) \\
\hline Q9H6F5 & ALVEFESNPEETREPGSPPSVQR & Phospho(ST) \\
\hline O75821 & GIPLATGDTSPEPELLPGAPLPPPK & Phospho(ST) \\
\hline O43719 & LFDDSDER & Phospho(ST) \\
\hline O43719 & VLDEEGSER & Phospho(ST) \\
\hline O43719 & LFEESDDKEDEDADGK & Phospho(ST) \\
\hline O43719 & VFDDESDEKEDEEYADEK & Phospho(ST) \\
\hline O43719 & EFDEDSDEKEEEEDTYEK & Phospho(ST) \\
\hline O43719 & VLDEEGSEREFDEDSDEKEEEEDTYEK & 2 Phospho(ST) \\
\hline P20700 & LKLSPSPSSR & Phospho(ST) \\
\hline P20700 & AGGPTTPLSPTR & 2 Phospho(ST) \\
\hline Q9Y2W1 & ASAVSELSPR & Phospho(ST) \\
\hline Q9Y2W1 & IDISPSTFR & Phospho(ST) \\
\hline Q9Y2W1 & NKKSPEIHR & Phospho(ST) \\
\hline Q9Y2W1 & GSFSDTGLGDGK & Phospho(ST) \\
\hline Q9Y2W1 & RIDISPSTFR & Phospho(ST) \\
\hline Q9Y2W1 & FSGEEGEIEDDESGTENR & Phospho(ST) \\
\hline P09651 & SESPKEPEQLR & Phospho(ST) \\
\hline P09651 & NQGGYGGSSSSSSYGSGR & Phospho(ST) \\
\hline P11388 & TQMAEVLPSPR & Phospho(ST) \\
\hline P11388 & SVVSDLEADDVK & Phospho(ST) \\
\hline P11388 & YLEESDEDDLF & Phospho(ST) \\
\hline P11388 & NENTEGSPQEDGVELEGLK & Phospho(ST) \\
\hline P11388 & VPDEEENEESDNEKETEK & Phospho(ST) \\
\hline P11388 & IKNENTEGSPQEDGVELEGLK & Phospho(ST) \\
\hline P11388 & GSVPLSSSPPATHFPDETEITNPVPK & Phospho(ST) \\
\hline P11388 & $\begin{array}{l}\text { FTMDLDSDEDFSDFDEKTDDEDFVPSDASP } \\
\text { PK }\end{array}$ & 3 Phospho(ST) \\
\hline O00193 & SASPDDDLGSSNWEAADLGNEER & Phospho(ST) \\
\hline O00193 & RSASPDDDLGSSNWEAADLGNEER & Phospho(ST) \\
\hline Q7Z417 & RTSPQVLGSILK & Phospho(ST) \\
\hline Q7Z417 & NLSSDEATNPISR & Phospho(ST) \\
\hline Q7Z417 & GADNDGSGSESGYTTPK & Phospho(ST) \\
\hline Q7Z417 & DYEIESQNPLASPTNTLLGSAK & Phospho(ST) \\
\hline P55081 & IVEPEVVGESDSEVEGDAWR & 2 Phospho(ST) \\
\hline P55081 & KIVEPEVVGESDSEVEGDAWR & 2 Phospho(ST) \\
\hline P55081 & SLAALDALNTDDENDEEEYEAWK & Phospho(ST) \\
\hline P55081 & RPDYAPMESSDEEDEEFQFIK & 2 Phospho(ST) \\
\hline P55081 & RPDYAPMESSDEEDEEFQFIK & Oxidation(M); \\
\hline
\end{tabular}




\begin{tabular}{|c|c|c|c|}
\hline Protein & Peptide sequence & Variable modification & \\
\hline & & 2 Phospho(ST) & \\
\hline P55081 & MEREDSSEEEEEEIDDEEIERR & 2 Phospho(ST) & \\
\hline O00264 & EGEEPTVYSDEEEPK & Phospho(ST) & \\
\hline O00264 & GDQPAASGDSDDDEPPPLPR & Phospho(ST) & \\
\hline O00264 & EGEEPTVYSDEEEPKDESAR & Phospho(ST) & \\
\hline O00264 & IVRGDQPAASGDSDDDEPPPLPR & Phospho(ST) & \\
\hline P54105 & FEEESKEPVADEEEEDSDDDVEPITEFR & Phospho(ST) & \\
\hline P18583 & RRSFSISPVR & 3 Phospho(ST) & \\
\hline P18583 & ESDQTLAALLSPK & Phospho(ST) & \\
\hline P18583 & EMEHNTVCAAGTSPVGEIGEEK & Phospho(ST) & \\
\hline \multirow[t]{2}{*}{ P18583 } & EMEHNTVCAAGTSPVGEIGEEK & Oxidation(M); & \\
\hline & & Phospho(ST) & \\
\hline Q12888 & GGPGKLSPR & Phospho(ST) & \\
\hline Q12888 & LITSEEERSPAK & Phospho(ST) & \\
\hline Q12888 & NSPEDLGLSLTGDSCK & Phospho(ST) & \\
\hline Q12888 & STPFIVPSSPTEQEGR & Phospho(ST) & \\
\hline \multirow[t]{2}{*}{ Q12888 } & MVIQGPSSPQGEAMVTDVLEDQK & Oxidation(M); & \\
\hline & & Phospho(ST) & \\
\hline Q12888 & IDEDGENTQIEDTEPMSPVLNSK & Phospho(ST) & \\
\hline P46013 & DINTFLGTPVQK & Phospho(ST) & \\
\hline P46013 & ITEVSCKSPQPESFK & Phospho(ST) & \\
\hline P46013 & IPCKSSPELEDTATSSK & Phospho(ST) & \\
\hline P46013 & IPCESPPLEVVDTTASTK & Phospho(ST) & \\
\hline P46013 & MPCESSPPESADTPTSTR & Phospho(ST) & \\
\hline P46013 & SGGSGHAVAEPASPEQELDQNK & Phospho(ST) & \\
\hline Q01105 & RQSPLPPQKK & Phospho(ST) & \\
\hline Q14247 & LPSSPVYEDAASFK & Phospho(ST) & \\
\hline Q14247 & TQTPPVSPAPQPTEER & Phospho(ST) & \\
\hline Q14247 & TQTPPVSPAPQPTEER & 2 Phospho(ST) & \\
\hline Q9BWU0 & MLGEDSDEEEEMDTSER & Phospho(ST) & \\
\hline Q9BWU0 & NWEDEDFYDSDDDTFLDR & Phospho(ST) & \\
\hline P19338 & ALVATPGK & Phospho(ST) & \\
\hline P19338 & KVAVATPAK & Phospho(ST) & \\
\hline P19338 & KVVVSPTKK & Phospho(ST) & \\
\hline \multirow[t]{2}{*}{ P19338 } & EVEEDSEDEEMSEDEEDDSSGEEVVIPQKK & Oxidation(M); & 3 \\
\hline & & Phospho(ST) & \\
\hline \multirow[t]{2}{*}{ P19338 } & EVEEDSEDEEMSEDEEDDSSGEEVVIPQKK & Oxidation(M); & \\
\hline & & 4 Phospho(ST) & \\
\hline P16949 & ESVPEFPLSPPK & Phospho(ST) & \\
\hline P16949 & ASGQAFELILSPR & Phospho(ST) & \\
\hline P16949 & SKESVPEFPLSPPK & Phospho(ST) & \\
\hline P27816 & SPSTLLPK & Phospho(ST) & \\
\hline P27816 & SKVGSTENIK & Phospho(ST) & \\
\hline
\end{tabular}




\begin{tabular}{|c|c|c|}
\hline Protein & Peptide sequence & Variable modification \\
\hline P27816 & RASPSKPASAPASR & Phospho(ST) \\
\hline P27816 & DMSPLSETEMALGK & Phospho(ST) \\
\hline P27816 & DVTPPPETEVVLIK & Phospho(ST) \\
\hline P27816 & DMESPTKLDVTLAK & $\begin{array}{l}\text { Oxidation(M); } \\
\text { Phospho(ST) }\end{array}$ \\
\hline P27816 & DMSPLSETEMALGK & 2 Phospho(ST) \\
\hline P27816 & KCSLPAEEDSVLEK & Phospho(ST) \\
\hline P07355 & GLGTDEDSLIEIICSR & Phospho(ST) \\
\hline A6NMY6 & GLGTDEDSLIEIICSR & Phospho(ST) \\
\hline Q9NYL9 & DLDEDELLGNLSETELK & Phospho(ST) \\
\hline Q9NYL9 & YKDLDEDELLGNLSETELK & Phospho(ST) \\
\hline Q5H9R7 & IQQFDDGGSDEEDIWEEK & Phospho(ST) \\
\hline Q14160 & MKSLEQDALR & Phospho(ST) \\
\hline Q14160 & VQSPEPPAPER & Phospho(ST) \\
\hline Q14160 & NSLESISSIDR & Phospho(ST) \\
\hline Q14160 & LPLLPPESPGPLR & Phospho(ST) \\
\hline Q14160 & QSPASPPPLGGGAPVR & Phospho(ST) \\
\hline Q14160 & RSEACPCQPDSGSPLPAEEEK & Phospho(ST) \\
\hline Q8NHW5 & EESEESDEDMGFGLFD & 2 Phospho(ST) \\
\hline Q8NHW5 & EESEESDEDMGFGLFD & $\begin{array}{l}\text { Oxidation(M); } \\
2 \text { Phospho(ST) }\end{array}$ \\
\hline Q8NHW5 & VEAKEESEESDEDMGFGLFD & 2 Phospho(ST) \\
\hline Q8NHW5 & VEAKEESEESDEDMGFGLFD & $\begin{array}{l}\text { Oxidation(M); } \\
2 \text { Phospho(ST) }\end{array}$ \\
\hline Q9H3N1 & VEEEQEADEEDVSEEEAESK & Phospho(ST) \\
\hline Q9H3N1 & KVEEEQEADEEDVSEEEAESK & Phospho(ST) \\
\hline O75475 & AVDITTPK & Phospho(ST) \\
\hline O75475 & ETSVSKEDTDHEEK & Phospho(ST) \\
\hline O75475 & TGVTSTSDSEEEGDDQEGEK & 2 Phospho(ST) \\
\hline O75475 & TGVTSTSDSEEEGDDQEGEK & 3 Phospho(ST) \\
\hline O75475 & TGVTSTSDSEEEGDDQEGEKK & 2 Phospho(ST) \\
\hline O75475 & QVETEEAGVVTTATASVNLKVSPK & Phospho(ST) \\
\hline Q16637 & GTGQSDDSDIWDDTALIK & 2 Phospho(ST) \\
\hline Q16637 & RGTGQSDDSDIWDDTALIK & Phospho(ST) \\
\hline Q16637 & RGTGQSDDSDIWDDTALIK & 2 Phospho(ST) \\
\hline P67809 & NYQQNYQNSESGEK & Phospho(ST) \\
\hline P67809 & AADPPAENSSAPEAEQGGAE & Phospho(ST) \\
\hline P67809 & NYQQNYQNSESGEKNEGSESAPEGQAQQR & Phospho(ST) \\
\hline O60716 & VGGSSVDLHR & Phospho(ST) \\
\hline O60716 & SQSSHSYDDSTLPLIDR & Phospho(ST) \\
\hline O60716 & HYEDGYPGGSDNYGSLSR & Phospho(ST) \\
\hline O60716 & TVQPVAMGPDGLPVDASSVSNNYIQTLGR & Phospho(ST) \\
\hline Q5T200 & TLTPPLR & Phospho(ST) \\
\hline
\end{tabular}




\begin{tabular}{|c|c|c|}
\hline Protein & Peptide sequence & Variable modification \\
\hline Q5T200 & KEVSPEVVR & Phospho(ST) \\
\hline Q5T200 & LRSPSNDSAHR & Phospho(ST) \\
\hline Q5T200 & KRTPSPSYQR & 2 Phospho(ST) \\
\hline Q5T200 & GNIETTSEDGQVFSPK & Phospho(ST) \\
\hline Q5T200 & SASPYPSHSLSSPQR & $\begin{array}{l}2 \text { Phospho(ST); } \\
\text { Phospho(Y) }\end{array}$ \\
\hline Q5T200 & SKGDSDISDEEAAQQSK & 2 Phospho(ST) \\
\hline Q5T200 & GPRTPSPPPPIPEDIALGK & 2 Phospho(ST) \\
\hline Q1KMD3 & SKSPLPPEEEAK & Phospho(ST) \\
\hline Q1KMD3 & SGDETPGSEVPGDK & Phospho(ST) \\
\hline Q1KMD3 & EEDEPEERSGDETPGSEVPGDK & Phospho(ST) \\
\hline Q1KMD3 & AAEEQGDDQDSEKSKPAGSDGER & Phospho(ST) \\
\hline Q1KMD3 & REEDEPEERSGDETPGSEVPGDK & Phospho(ST) \\
\hline Q1KMD3 & $\begin{array}{l}\text { SKSPLPPEEEAKDEEEDQTLVNLDTYTSDL } \\
\text { HFQVSK }\end{array}$ & Phospho(ST) \\
\hline Q02952 & LSAEYEK & Phospho(ST) \\
\hline Q02952 & EGVTPWASFK & Phospho(ST) \\
\hline Q02952 & VVGQTTPESFEK & Phospho(ST) \\
\hline Q02952 & RPSESDKEDELDKVK & Phospho(ST) \\
\hline Q02952 & GLAEVQQDGEAEEGATSDGEK & Phospho(ST) \\
\hline Q14103 & IDASKNEEDEGHSNSSPR & Phospho(ST) \\
\hline Q9UQ88 & EYGSPLK & Phospho(ST) \\
\hline Q9UQ88 & RDSLEEGELR & Phospho(ST) \\
\hline Q9UQ88 & DLLSDLQDISDSER & Phospho(ST) \\
\hline Q9UQ88 & DLLSDLQDISDSER & 2 Phospho(ST) \\
\hline Q9UQ88 & GTSPRPPEGGLGYSQLGDDDLK & Phospho(ST) \\
\hline Q5SW79 & LGEASDSELADADK & Phospho(ST) \\
\hline Q5SW79 & HDDGTQSDSENAGAHR & Phospho(ST) \\
\hline P18858 & AETPTESVSEPEVATK & Phospho(ST) \\
\hline P18858 & EGEDGDQPTTPPKPLK & Phospho(ST) \\
\hline P18858 & TIQEVLEEQSEDEDR & Phospho(ST) \\
\hline P18858 & VLGSEGEEEDEALSPAK & 2 Phospho(ST) \\
\hline P18858 & TIQEVLEEQSEDEDREAK & Phospho(ST) \\
\hline Q9NR30 & AEPSEVDMNSPK & Phospho(ST) \\
\hline Q9NR30 & KAEPSEVDMNSPK & Phospho(ST) \\
\hline Q9NR30 & KKEEPSQNDISPK & Phospho(ST) \\
\hline Q9NR30 & NEEPSEEEIDAPKPK & Phospho(ST) \\
\hline O60841 & QSFDDNDSEELEDKDSK & Phospho(ST) \\
\hline O60841 & NKPGPNIESGNEDDDASFK & Phospho(ST) \\
\hline O60841 & KQSFDDNDSEELEDKDSK & Phospho(ST) \\
\hline O60841 & VEMYSGSDDDDDFNKLPK & Phospho(ST); Phospho(Y) \\
\hline O60841 & VEMYSGSDDDDDFNKLPK & $\begin{array}{l}\text { Oxidation(M); } \\
2 \text { Phospho(ST) }\end{array}$ \\
\hline
\end{tabular}




\begin{tabular}{|c|c|c|}
\hline Protein & Peptide sequence & Variable modification \\
\hline O60841 & INSSGESGDESDEFLQSR & 4 Phospho(ST) \\
\hline Q9UKV3 & TAQVPSPPR & Phospho(ST) \\
\hline Q9UKV3 & SSSISEEKGDSDDEKPR & Phospho(ST) \\
\hline Q13283 & SSSPAPADIAQTVQEDLR & Phospho(ST) \\
\hline Q13283 & YQDEVFGGFVTEPQEESEEEVEEPEER & Phospho(ST) \\
\hline P12956 & LGSLVDEFK & Phospho(ST) \\
\hline P12956 & IMATPEQVGK & Phospho(ST) \\
\hline Q29RF7 & IISVTPVK & Phospho(ST) \\
\hline Q29RF7 & AAVGQESPGGLEAGNAK & Phospho(ST) \\
\hline Q9Y2D5 & DALGDSLQVPVSPSSTTSSR & Phospho(ST) \\
\hline Q9Y2D5 & SPGALETPSAAGSQGNTASQGK & Phospho(ST) \\
\hline Q9Y2D5 & GQKSPGALETPSAAGSQGNTASQGK & Phospho(ST) \\
\hline Q9Y2D5 & VKPPPSPTTEGPSLQPDLAPEEAAGTQRPK & Phospho(ST) \\
\hline Q8WVC0 & KLTSDEEGEPSGK & Phospho(ST) \\
\hline Q8WVC0 & KYVISDEEEEDDD & Phospho(ST) \\
\hline Q8WVC0 & NAIASDSEADSDTEVPK & 3 Phospho(ST) \\
\hline Q8WVC0 & MQNTDDEERPQLSDDER & 2 Phospho(ST) \\
\hline O60343 & LGSVDSFER & Phospho(ST) \\
\hline O60343 & SLTSSLENIFSR & Phospho(ST) \\
\hline Q9H3Q1 & NAMSLPQLNEK & Phospho(ST) \\
\hline Q9H3Q1 & AGPDLPSLPSHALEDEGWAAAAPSPGSAR & Phospho(ST) \\
\hline Q9H3Q1 & AGPDLPSLPSHALEDEGWAAAAPSPGSAR & 2 Phospho(ST) \\
\hline Q96CX2 & SPSGGAAGPLLTPSQSLDGSR & Phospho(ST) \\
\hline P50502 & ADEPSSEESDLEIDK & 2 Phospho(ST) \\
\hline P50502 & ADEPSSEESDLEIDK & 3 Phospho(ST) \\
\hline P50502 & KVEEDLKADEPSSEESDLEIDK & 3 Phospho(ST) \\
\hline Q8IZP2 & ADEPSSEESDLEIDK & 2 Phospho(ST) \\
\hline Q8IZP2 & ADEPSSEESDLEIDK & 3 Phospho(ST) \\
\hline Q00839 & $\begin{array}{l}\text { AKSPQPPVEEEDEHFDDTVVCLDTYNCDL } \\
\text { HFK }\end{array}$ & Phospho(ST) \\
\hline Q9Y3T9 & EAARSPDKPGGSPSASR & Phospho(ST) \\
\hline Q9Y3T9 & DLFDLNSSEEDDTEGFSER & 2 Phospho(ST) \\
\hline Q9BQG0 & EIPSATQSPISK & Phospho(ST) \\
\hline Q9BQG0 & SPAPGAPTRSPSTPAK & Phospho(ST) \\
\hline Q9BQG0 & $\begin{array}{l}\text { ALGGEDSENEEELGDEAMMALDQSLASLF } \\
\text { AEQK }\end{array}$ & Phospho(ST) \\
\hline Q9BQG0 & $\begin{array}{l}\text { ALGGEDSENEEELGDEAMMALDQSLASLF } \\
\text { AEQK }\end{array}$ & $\begin{array}{l}\text { Oxidation(M); } \\
\text { Phospho(ST) }\end{array}$ \\
\hline O75592 & SYSVVASEYDK & Phospho(ST) \\
\hline O75592 & VNSGDTEVGSSLLR & Phospho(ST) \\
\hline O75592 & SLSPNHNTLQTLK & Phospho(ST) \\
\hline Q9UQE7 & KGDVEGSQSQDEGEGSGESER & Phospho(ST) \\
\hline O95361 & ETEEQDSDSAEQGDPAGEGK & Phospho(ST) \\
\hline
\end{tabular}




\begin{tabular}{|c|c|c|}
\hline Protein & Peptide sequence & Variable modification \\
\hline P22059 & MLAESDESGDEESVSQTDK & 2 Phospho(ST) \\
\hline P22059 & MLAESDESGDEESVSQTDKTELQNTLR & 2 Phospho(ST) \\
\hline P22059 & GDMSDEDDENEFFDAPEIITMPENLGHK & Phospho(ST) \\
\hline \multirow[t]{2}{*}{ P22059 } & GDMSDEDDENEFFDAPEIITMPENLGHK & Oxidation(M); \\
\hline & & Phospho(ST) \\
\hline P05783 & LLEDGEDFNLGDALDSSNSMQTIQK & Phospho(ST) \\
\hline P05783 & RLLEDGEDFNLGDALDSSNSMQTIQK & Phospho(ST) \\
\hline P13861 & GDSESEEDEDLEVPVPSR & 2 Phospho(ST) \\
\hline P13861 & RVSVCAETYNPDEEEEDTDPR & Phospho(ST) \\
\hline P13861 & VADAKGDSESEEDEDLEVPVPSR & 2 Phospho(ST) \\
\hline Q9GZR7 & AQAVSEEEEEEEGK & Phospho(ST) \\
\hline Q9GZR7 & KAQAVSEEEEEEEGK & Phospho(ST) \\
\hline Q14676 & LLLAEDSEEEVDFLSER & Phospho(ST) \\
\hline Q14676 & SQTTTERDSDTDVEEEELPVENR & 2 Phospho(ST) \\
\hline Q14676 & ESEDSETQPFDTHLEAYGPCLSPPR & Phospho(ST) \\
\hline Q14676 & AQPFGFIDSDTDAEEERIPATPVVIPMK & 2 Phospho(ST) \\
\hline P51991 & SSGSPYGGGYGSGGGSGGYGSR & Phospho(ST) \\
\hline P47712 & CSVSLSNVEAR & Phospho(ST) \\
\hline P47712 & HIVSNDSSDSDDESHEPK & Phospho(ST) \\
\hline P47712 & HIVSNDSSDSDDESHEPK & 2 Phospho(ST) \\
\hline P47712 & HIVSNDSSDSDDESHEPK & 3 Phospho(ST) \\
\hline P04792 & GPSWDPFR & Phospho(ST) \\
\hline P04792 & QLSSGVSEIR & Phospho(ST) \\
\hline P04792 & AQLGGPEAAKSDETAAK & Phospho(ST) \\
\hline Q08AD1 & SISNEGLTLNNSHVSK & Phospho(ST) \\
\hline Q08AD1 & SSSMSYVDGFIGTWPK & Phospho(ST) \\
\hline \multirow[t]{2}{*}{ Q08AD1 } & SSSMSYVDGFIGTWPK & Oxidation(M); \\
\hline & & Phospho(ST) \\
\hline Q08AD1 & YDGESDKEQFDDDQK & Phospho(ST) \\
\hline P00558 & ALESPERPFLAILGGAK & Phospho(ST) \\
\hline O00499 & SPSPPDGSPAATPEIR & Phospho(ST) \\
\hline O00499 & GNKSPSPPDGSPAATPEIR & Phospho(ST) \\
\hline O00499 & VNHEPEPAGGATPGATLPKSPSQLR & Phospho(ST) \\
\hline P35606 & STAQQELDGKPASPTPVIVASHTANK & Phospho(ST) \\
\hline P35606 & STAQQELDGKPASPTPVIVASHTANKEEK & Phospho(ST) \\
\hline Q13523 & KPIKSPSK & Phospho(ST) \\
\hline Q13523 & ARSPTDDKVK & Phospho(ST) \\
\hline Q13523 & KKSPIINESR & Phospho(ST) \\
\hline Q13439 & SSSKESLVR & Phospho(ST) \\
\hline Q13439 & TSSFTEQLDEGTPNR & Phospho(ST) \\
\hline Q13439 & EENPESDGEPVVEDGTSVK & Phospho(ST) \\
\hline Q9Y2X3 & EEPLSEEEPCTSTAIASPEK & Phospho(ST) \\
\hline Q9Y2X3 & HIKEEPLSEEEPCTSTAIASPEK & Phospho(ST) \\
\hline
\end{tabular}




\begin{tabular}{|c|c|c|}
\hline Protein & Peptide sequence & Variable modification \\
\hline Q9Y2X3 & HIKEEPLSEEEPCTSTAIASPEK & 2 Phospho(ST) \\
\hline Q9Y2X3 & HIKEEPLSEEEPCTSTAIASPEKK & Phospho(ST) \\
\hline Q9Y2X3 & HIKEEPLSEEEPCTSTAIASPEKK & 2 Phospho(ST) \\
\hline Q86WB0 & LPLVPESPR & Phospho(ST) \\
\hline Q86WB0 & SQDATFSPGSEQAEK & Phospho(ST) \\
\hline Q86WB0 & SMGTGDTPGLEVPSSPLRK & Phospho(ST) \\
\hline Q86WB0 & SWDSSSPVDRPEPEAASPTTR & Phospho(ST) \\
\hline O00566 & KSPVFSDEDSDLDFDISK & 3 Phospho(ST) \\
\hline O00566 & KDDNDEEEEDIDFFEDIDSDEDEGGLFGSK & Phospho(ST) \\
\hline Q8N1G4 & EEGSLSDTEADAVSGQLPDPTTNPSAGK & Phospho(ST) \\
\hline Q8N1G4 & $\begin{array}{l}\text { YTLENKEEGSLSDTEADAVSGQLPDPTTNP } \\
\text { SAGK }\end{array}$ & Phospho(ST) \\
\hline P35658 & VPAKLSPMK & Phospho(ST) \\
\hline P35658 & ITPPAAKPGSPQAK & Phospho(ST) \\
\hline P35658 & SPGSTPTTPTSSQAPQK & Phospho(ST) \\
\hline P35658 & SPGSTPTTPTSSQAPQK & 2 Phospho(ST) \\
\hline P28715 & NAPAAVDEGSISPR & Phospho(ST) \\
\hline P28715 & FDSSLLSSDDETK & Phospho(ST) \\
\hline Q08J23 & ESTQLSPADLTEGKPTDPSK & Phospho(ST) \\
\hline Q08J23 & $\begin{array}{l}\text { AGEPNSPDAEEANSPDVTAGCDPAGVHPP } \\
\mathrm{R}\end{array}$ & Phospho(ST) \\
\hline Q08J23 & $\begin{array}{l}\text { AGEPNSPDAEEANSPDVTAGCDPAGVHPP } \\
\mathrm{R}\end{array}$ & 2 Phospho(ST) \\
\hline P35221 & TPEELDDSDFETEDFDVR & Phospho(ST) \\
\hline Q12873 & FGTEELFK & Phospho(ST) \\
\hline Q12873 & METEADAPSPAPSLGER & Phospho(ST) \\
\hline Q12873 & ELQGDGPPSSPTNDPTVK & Phospho(ST) \\
\hline P53396 & TASFSESR & Phospho(ST) \\
\hline P53396 & AKPAMPQDSVPSPR & Phospho(ST) \\
\hline P04075 & PYQYPALTPEQK & Phospho(ST) \\
\hline Q9Y5J1 & VQEHEDSGDSEVENEAK & 2 Phospho(ST) \\
\hline Q9Y5J1 & KTSSDDESEEDEDDLLQR & 2 Phospho(ST) \\
\hline Q9Y5J1 & KTSSDDESEEDEDDLLQR & 3 Phospho(ST) \\
\hline P25788 & ESLKEEDESDDDNM & $\begin{array}{l}\text { Oxidation(M); } \\
\text { Phospho(ST) }\end{array}$ \\
\hline Q96ST2 & AAVLSDSEDEEK & 2 Phospho(ST) \\
\hline Q96ST2 & KAAVLSDSEDEEK & 2 Phospho(ST) \\
\hline Q96ST2 & AAVLSDSEDEEKASAK & 2 Phospho(ST) \\
\hline Q96ST2 & VVSDADDSDSDAVSDK & 3 Phospho(ST) \\
\hline Q96ST2 & NQASDSENEELPKPR & 2 Phospho(ST) \\
\hline Q96ST2 & HQASDSENEEPPKPR & 2 Phospho(ST) \\
\hline P16401 & AKKPAGATPK & Phospho(ST) \\
\hline P16401 & SETAPAETATPAPVEKSPAK & Phospho(ST) \\
\hline
\end{tabular}




\begin{tabular}{|c|c|c|}
\hline Protein & Peptide sequence & Variable modification \\
\hline P16401 & SETAPAETATPAPVEKSPAKK & Phospho(ST) \\
\hline P24928 & YSPTSPTYSPTSPK & Phospho(ST) \\
\hline P24928 & YSPTSPTYSPTTPK & Phospho(ST) \\
\hline P24928 & YSPTSPTYSPTSPVYTPTSPK & Phospho(ST) \\
\hline P24928 & YTPTSPSYSPSSPEYTPTSPK & Phospho(ST) \\
\hline Q13085 & SSMSGLHLVK & Phospho(ST) \\
\hline Q13085 & FIIGSVSEDNSEDEISNLVK & Phospho(ST) \\
\hline Q13085 & FIIGSVSEDNSEDEISNLVK & 2 Phospho(ST) \\
\hline P52756 & GLVAAYSGDSDNEEELVER & Phospho(ST) \\
\hline O60271 & SASQSSLDKLDQELK & 2 Phospho(ST) \\
\hline Q13425 & GLGPPSPPAPPR & Phospho(ST) \\
\hline Q13425 & GPAGEAGASPPVR & Phospho(ST) \\
\hline Q13425 & GPAGEAGASPPVRR & Phospho(ST) \\
\hline Q13425 & KPSLVSDLPWEGAAPQSPSFSGSEDSGSPK & Phospho(ST) \\
\hline P10644 & EDEISPPPPNPVVK & Phospho(ST) \\
\hline P10644 & TDSREDEISPPPPNPVVK & Phospho(ST) \\
\hline Q96D46 & DSAIPVESDTDDEGAPR & Phospho(ST) \\
\hline Q96D46 & DSAIPVESDTDDEGAPR & 2 Phospho(ST) \\
\hline Q9Y4F1 & LGAPENSGISTLER & Phospho(ST) \\
\hline Q9Y4F1 & VSAGEPGSHPSPAPR & Phospho(ST) \\
\hline Q9Y4F1 & SSSPAPEFLASSPPDNK & Phospho(ST) \\
\hline Q15233 & FGQAATMEGIGAIGGTPPAFNR & Phospho(ST) \\
\hline Q15233 & FGQAATMEGIGAIGGTPPAFNR & $\begin{array}{l}\text { Oxidation(M); } \\
\text { Phospho(ST) }\end{array}$ \\
\hline O60763 & DLGHPVEEEDELESGDQEDEDDESEDPGK & Phospho(ST) \\
\hline O60763 & $\begin{array}{l}\text { DLGHPVEEEDELESGDQEDEDDESEDPGK } \\
\text { DLDHI }\end{array}$ & Phospho(ST) \\
\hline P60174 & KQSLGELIGTLNAAK & Phospho(ST) \\
\hline Q92598 & IESPKLER & Phospho(ST) \\
\hline O00505 & NVPQEESLEDSDVDADFK & Phospho(ST) \\
\hline O95817 & SSTPLHSPSPIR & 2 Phospho(ST) \\
\hline O95817 & SQSPAASDCSSSSSSASLPSSGR & Phospho(ST) \\
\hline O95817 & VPPAPVPCPPPSPGPSAVPSSPK & Phospho(ST) \\
\hline Q9UK76 & RNSSEASSGDFLDLK & Phospho(ST) \\
\hline P17096 & EEEEGISQESSEEEQ & 2 Phospho(ST) \\
\hline P17096 & LEKEEEEGISQESSEEEQ & 2 Phospho(ST) \\
\hline P17096 & KLEKEEEEGISQESSEEEQ & Phospho(ST) \\
\hline P17096 & KLEKEEEEGISQESSEEEQ & 2 Phospho(ST) \\
\hline P17096 & KLEKEEEEGISQESSEEEQ & 3 Phospho(ST) \\
\hline P17096 & KQPPVSPGTALVGSQKEPSEVPTPK & Phospho(ST) \\
\hline Q96T23 & KKPDSPPK & Phospho(ST) \\
\hline Q96T23 & DAQRLSPIPEEVPK & Phospho(ST) \\
\hline Q96T23 & IESDEEEDFENVGK & Phospho(ST) \\
\hline
\end{tabular}




\begin{tabular}{|c|c|c|}
\hline Protein & Peptide sequence & Variable modification \\
\hline Q96T23 & TFLDKDAQRLSPIPEEVPK & Phospho(ST) \\
\hline Q96T23 & STLESEKPGSPEAAETSPPSNIIDHCEK & 2 Phospho(ST) \\
\hline P02765 & CDSSPDSAEDVR & Phospho(ST) \\
\hline Q9NY27 & NHSDSSTSESEVSSVSPLK & Phospho(ST) \\
\hline O95425 & DSSFTEVPRSPK & Phospho(ST) \\
\hline O95425 & YQTQPVTLGEVEQVQSGK & Phospho(ST) \\
\hline Q9Y6X9 & AKASEESLR & Phospho(ST) \\
\hline Q9Y6X9 & SVAVSDEEEVEEEAER & Phospho(ST) \\
\hline Q71DI3 & STELLIRK & Phospho(ST) \\
\hline P68431 & STELLIRK & Phospho(ST) \\
\hline P41236 & IQEQESSGEEDSDLSPEER & Phospho(ST) \\
\hline P41236 & IQEQESSGEEDSDLSPEER & 2 Phospho(ST) \\
\hline P41236 & $\begin{array}{l}\text { IDEPSTPYHSMMGDDEDACSDTEATEAMA } \\
\text { PDILAR }\end{array}$ & Phospho(ST) \\
\hline Q6NXS1 & IQEQESSGEEDSDLSPEER & Phospho(ST) \\
\hline Q6NXS1 & IQEQESSGEEDSDLSPEER & 2 Phospho(ST) \\
\hline O75643 & EEASDDDMEGDEAVVR & Phospho(ST) \\
\hline P25205 & DGDSYDPYDFSDTEEEMPQVHTPK & Phospho(ST) \\
\hline P25205 & DGDSYDPYDFSDTEEEMPQVHTPK & $\begin{array}{l}\text { Oxidation(M); } \\
\text { Phospho(ST) }\end{array}$ \\
\hline P25205 & DGDSYDPYDFSDTEEEMPQVHTPK & 2 Phospho(ST) \\
\hline Q9HAU0 & TNSMQQLEQWIK & Phospho(ST) \\
\hline Q9HAU0 & GLNVIGASDQSPLQSPSNLR & 2 Phospho(ST) \\
\hline Q7Z460 & VLSTSTDLEAAVADALK & Phospho(ST) \\
\hline Q7Z460 & RQSSGSATNVASTPDNR & Phospho(ST) \\
\hline P49006 & LSGLSFK & Phospho(ST) \\
\hline Q04695 & QFTSSSSIK & Phospho(ST) \\
\hline Q04695 & LSGGLGAGSCR & Phospho(ST) \\
\hline Q9C075 & SSMKVSATPKIK & 3 Phospho(ST) \\
\hline P0C0S8 & KTESHHK & Phospho(ST) \\
\hline Q9NZN4 & GPDEAMEDGEEGSDDEAEWVVTK & Phospho(ST) \\
\hline P23588 & SLENETLNKEEDCHSPTSKPPKPDQPLK & Phospho(ST) \\
\hline Q04637 & KAASLTEDR & Phospho(ST) \\
\hline Q04637 & EAEEESDHN & Phospho(ST) \\
\hline Q04637 & EAALPPVSPLK & Phospho(ST) \\
\hline Q96I25 & RPDPDSDEDEDYER & Phospho(ST) \\
\hline P46379 & LQEDPNYSPQR & Phospho(ST) \\
\hline P46379 & ENASPAPGTTAEEAMSR & Phospho(ST) \\
\hline P46379 & ENASPAPGTTAEEAMSR & $\begin{array}{l}\text { Oxidation(M); } \\
\text { Phospho(ST) }\end{array}$ \\
\hline P46379 & $\begin{array}{l}\text { APPQTHLPSGASSGTGSASATHGGGSPPGT } \\
\mathrm{R}\end{array}$ & Phospho(ST) \\
\hline Q15424 & SEPVKEESSELEQPFAQDTSSVGPDRK & Phospho(ST) \\
\hline
\end{tabular}




\begin{tabular}{|c|c|c|}
\hline Protein & Peptide sequence & Variable modification \\
\hline Q14151 & SEPVKEESSELEQPFAQDTSSVGPDRK & Phospho(ST) \\
\hline P35659 & EESEEEEDEDDEEEEEEEK & Phospho(ST) \\
\hline Q01082 & AQTLPTSVVTITSESSPGKR & Phospho(ST) \\
\hline Q9NTJ3 & TESPATAAETASEELDNR & Phospho(ST) \\
\hline Q9NTJ3 & EEGPPPPSPDGASSDAEPEPPSGR & Phospho(ST) \\
\hline Q9NTJ3 & EEGPPPPSPDGASSDAEPEPPSGR & 2 Phospho(ST) \\
\hline O94906 & LSQVSDSVSGQTVVDPK & Phospho(ST) \\
\hline Q9ULF5 & LNETELTDLEGQQESPPK & Phospho(ST) \\
\hline Q9ULF5 & LNETELTDLEGQQESPPKNYLCIEEEK & Phospho(ST) \\
\hline Q9C0C2 & EVLASPDR & Phospho(ST) \\
\hline Q9C0C2 & VPSSDEEVVEEPQSR & 2 Phospho(ST) \\
\hline Q9C0C2 & TEAQDLCRASPEPPGPESSSR & Phospho(ST) \\
\hline Q9C0C2 & SPSQDFSFIEDTEILDSAMYR & Phospho(ST) \\
\hline Q9C0C2 & SFGTRPLSSGFSPEEAQQQDEEFEK & Phospho(ST) \\
\hline Q96T37 & DGWSLDR & Phospho(ST) \\
\hline Q96T37 & DRTPPLLYR & Phospho(ST) \\
\hline Q96T37 & SLSPGGAALGYR & Phospho(ST) \\
\hline Q96T37 & HCAPSPDRSPELSSSR & 2 Phospho(ST) \\
\hline Q96T37 & SRSPLDKDTYPPSASVVGASVGGHR & 2 Phospho(ST) \\
\hline Q69YN4 & SFLSEPSSPGR & Phospho(ST) \\
\hline Q69YN4 & VISHDRDSPPPPPPPPPPPQPQPSLK & Phospho(ST) \\
\hline O95747 & TEDGGWEWSDDEFDEESEEGK & Phospho(ST) \\
\hline P56211 & VTSPEKAEEAK & Phospho(ST) \\
\hline P56211 & YFDSGDYNMAK & Phospho(ST) \\
\hline O43768 & YFDSGDYNMAK & Phospho(ST) \\
\hline Q86TC9 & TPEPSSPVKEPPPVLAKPK & Phospho(ST) \\
\hline Q86TC9 & TPVDESDDEIQHDEIPTGK & Phospho(ST) \\
\hline Q9ULX3 & KDDSDDDGGGWITPSNIK & Phospho(ST) \\
\hline Q14432 & VNPVTSLSENYTCSDSEESSEKDK & Phospho(ST) \\
\hline Q9Y6D5 & GQSQLSNPTDDSWK & Phospho(ST) \\
\hline Q9Y6D5 & ELEKPIQSKPQSPVIQAAAVSPK & Phospho(ST) \\
\hline O75122 & SRSDIDVNAAAGAK & Phospho(ST) \\
\hline Q6P158 & DLQEQDADAGSER & Phospho(ST) \\
\hline \multirow[t]{2}{*}{ Q6P158 } & DCSMVSVYPLVLFGGGQVNVQLQR & Oxidation(M); \\
\hline & & Phospho(Y) \\
\hline Q9Y4H2 & HNSASVENVSLR & Phospho(ST) \\
\hline Q9Y4H2 & GVPGCCYSSLPR & Phospho(ST) \\
\hline Q9Y4H2 & KSSEGGVGVGPGGGDEPPTSPR & Phospho(ST) \\
\hline O15027 & QIDSSPVGGETDETTVSQNYR & Phospho(ST) \\
\hline Q9UQB8 & SSSMAAGLER & Phospho(ST) \\
\hline Q9UQB8 & LSDSYSNTLPVR & Phospho(ST) \\
\hline P10412 & KAPKSPAK & Phospho(ST) \\
\hline P10412 & KASGPPVSELITK & Phospho(ST) \\
\hline
\end{tabular}




\begin{tabular}{|c|c|c|}
\hline Protein & Peptide sequence & Variable modification \\
\hline P10412 & SETAPAAPAAPAPAEKTPVK & Phospho(ST) \\
\hline P16402 & KVKTPQPK & Phospho(ST) \\
\hline P16402 & KASGPPVSELITK & Phospho(ST) \\
\hline Q13501 & LTPVSPESSSTEEK & Phospho(ST) \\
\hline Q13501 & SRLTPVSPESSSTEEK & Phospho(ST) \\
\hline Q13501 & SRLTPVSPESSSTEEK & 2 Phospho(ST) \\
\hline Q13501 & $\begin{array}{l}\text { EVDPSTGELQSLQMPESEGPSSLDPSQEGPT } \\
\text { GLK }\end{array}$ & Phospho(ST) \\
\hline Q13547 & IACEEEFSDSEEEGEGGR & 2 Phospho(ST) \\
\hline Q13547 & IACEEEFSDSEEEGEGGRK & 2 Phospho(ST) \\
\hline Q13547 & $\begin{array}{l}\text { MLPHAPGVQMQAIPEDAIPEESGDEDEDDP } \\
\text { DKR }\end{array}$ & Phospho(ST) \\
\hline Q13547 & $\begin{array}{l}\text { MLPHAPGVQMQAIPEDAIPEESGDEDEDDP } \\
\text { DKR }\end{array}$ & $\begin{array}{l}\text { Oxidation(M); } \\
\text { Phospho(ST) }\end{array}$ \\
\hline Q8WX93 & SPSGHPHVR & Phospho(ST) \\
\hline Q8WX93 & SLPTPAVLLSPTK & Phospho(ST) \\
\hline Q8WX93 & SRSRDSGDENEPIQER & 3 Phospho(ST) \\
\hline Q8WX93 & $\begin{array}{l}\text { QFIAAQNLGPASGHGTPASSPSSSSLPSPMS } \\
\text { PTPR }\end{array}$ & Phospho(ST) \\
\hline Q6PD62 & RKGSGSEQEGEDEEGGER & 2 Phospho(ST) \\
\hline Q6PD62 & KGGEFDEFVNDDTDDDLPISK & Phospho(ST) \\
\hline Q641Q2 & RTPSDDEEDNLFAPPK & Phospho(ST) \\
\hline Q641Q2 & GLFSDEEDSEDLFSSQSASK & Phospho(ST) \\
\hline Q9Y4E1 & RTPSDDEEDNLFAPPK & Phospho(ST) \\
\hline O15534 & GLDSSSTAPSALGER & Phospho(ST) \\
\hline Q8IVT2 & ALSSDSILSPAPDAR & Phospho(ST) \\
\hline Q8IVT2 & RALSSDSILSPAPDAR & Phospho(ST) \\
\hline Q8IVT2 & ASTPDWVSEGPQPGLR & Phospho(ST) \\
\hline P17252 & STLNPQWNESFTFK & Phospho(ST) \\
\hline P17252 & TFCGTPDYIAPEIIAYQPYGK & Phospho(ST) \\
\hline P05129 & TFCGTPDYIAPEIIAYQPYGK & Phospho(ST) \\
\hline P20810 & SESELIDELSEDFDR & Phospho(ST) \\
\hline Q13112 & TQDPSSPGTTPPQAR & Phospho(ST) \\
\hline Q13112 & GSSPGPRPVEGTPASR & Phospho(ST) \\
\hline Q13112 & TQDPSSPGTTPPQAR & 2 Phospho(ST) \\
\hline Q7Z6Z7 & DGGSGNSTIIVSR & 2 Phospho(ST) \\
\hline Q7Z6Z7 & GSGTASDDEFENLR & Phospho(ST) \\
\hline Q7Z6Z7 & LLGPSAAADILQLSSSLPLQSR & Phospho(ST) \\
\hline Q7Z6Z7 & $\begin{array}{l}\text { REESPMDVDQPSPSAQDTQSIASDGTPQGE } \\
\mathrm{K}\end{array}$ & Phospho(ST) \\
\hline Q96EZ8 & GDQVLNFSDAEDLIDDSK & Phospho(ST) \\
\hline Q96EV2 & AADEDWDSELEDDLLGEDLLSGK & Phospho(ST) \\
\hline Q96EV2 & AADEDWDSELEDDLLGEDLLSGKK & Phospho(ST) \\
\hline
\end{tabular}




\begin{tabular}{|c|c|c|}
\hline Protein & Peptide sequence & Variable modification \\
\hline Q9UJU6 & AMSTTSISSPQPGK & Phospho(ST) \\
\hline \multirow[t]{2}{*}{ Q9UJU6 } & AMSTTSISSPQPGK & Oxidation(M); \\
\hline & & Phospho(ST) \\
\hline Q5JTD0 & GSPEEELPLPAFEK & Phospho(ST) \\
\hline Q5JTD0 & KDSLTQAQEQGNLLN & Phospho(ST) \\
\hline P17612 & TWTLCGTPEYLAPEIILSK & Phospho(ST) \\
\hline Q5JSH3 & ELSDQATASPIVAR & Phospho(ST) \\
\hline Q5JSH3 & LTQTSSTEQLNVLETETEVLNK & Phospho(ST) \\
\hline Q9UPQ0 & SDSLSPPR & Phospho(ST) \\
\hline Q9UPQ0 & SINHQIESPSER & Phospho(ST) \\
\hline Q9UPQ0 & GSSDGRGSDSESDLPHR & Phospho(ST) \\
\hline Q9UPQ0 & VVKPKSPEPEATLTFPFLDK & Phospho(ST) \\
\hline Q13610 & EKLQEEGGGSDEEETGSPSEDGMQSAR & Phospho(ST) \\
\hline Q14684 & VGDGDLSAEEIPENEVSLR & Phospho(ST) \\
\hline P52948 & SLVGGLLQSK & Phospho(ST) \\
\hline P52948 & YGLQDSDEEEEEHPSK & Phospho(ST) \\
\hline Q5JTH9 & GDSIEEILADSEDEEDNEEEER & Phospho(ST) \\
\hline Q6KC79 & AITSLLGGGSPK & Phospho(ST) \\
\hline Q6KC79 & AAMYDIISSPSKDSTK & Phospho(ST) \\
\hline Q8WWM7 & GINGGPSRMSPK & Phospho(ST) \\
\hline Q8WWM7 & EVDGLLTSEPMGSPVSSK & Phospho(ST) \\
\hline Q8WWM7 & LQPSSSPENSLDPFPPR & Phospho(ST) \\
\hline Q8WWM7 & EKEVDGLLTSEPMGSPVSSK & Phospho(ST) \\
\hline Q8WWM7 & $\begin{array}{l}\text { EDKPPLAPSGGTEGPEQPPPPCPSQTGSPPV } \\
\text { GLIK }\end{array}$ & Phospho(ST) \\
\hline Q9H2P0 & LMHNASDSEVDQDDVVEWK & 2 Phospho(ST) \\
\hline Q0ZGT2 & TISQEFLTPGK & Phospho(ST) \\
\hline Q0ZGT2 & EMLASDDEEDVSSK & Phospho(ST) \\
\hline Q0ZGT2 & EMLASDDEEDVSSKVEK & Phospho(ST) \\
\hline Q9NVU0 & EAANEAGDSSQDEAEDDVK & 2 Phospho(ST) \\
\hline Q9NVU0 & $\begin{array}{l}\text { IKEEPVSEEGEEDEEQEAEEEPMDTSPSGLH } \\
\text { SK }\end{array}$ & Phospho(ST) \\
\hline Q96TC7 & SQSLPNSLDYTQTSDPGR & Phospho(ST) \\
\hline O14639 & TLSPTPSAEGYQDVR & Phospho(ST) \\
\hline P35611 & TPSFLKK & Phospho(ST) \\
\hline P35611 & SPGSPVGEGTGSPPK & Phospho(ST) \\
\hline Q3KQU3 & LSASTASELSPK & Phospho(ST) \\
\hline Q3KQU3 & SSQPSPTAVPASDSPPTK & Phospho(ST) \\
\hline O43399 & GLLSDSMTDVPVDTGVAAR & Phospho(ST) \\
\hline P07814 & LKAEKSPK & Phospho(ST) \\
\hline P07814 & EYIPGQPPLSQSSDSSPTR & Phospho(ST) \\
\hline P07814 & EYIPGQPPLSQSSDSSPTR & 2 Phospho(ST) \\
\hline Q86UE4 & SETSWESPK & Phospho(ST) \\
\hline
\end{tabular}




\begin{tabular}{|c|c|c|}
\hline Protein & Peptide sequence & Variable modification \\
\hline Q86UE4 & LSSQISAGEEK & Phospho(ST) \\
\hline Q86UE4 & SQEPIPDDQKVSDDDKEK & Phospho(ST) \\
\hline Q15811 & SAFTPATATGSSPSPVLGQGEK & Phospho(ST) \\
\hline Q14692 & LGPQNFIDETSDIENLLK & Phospho(ST) \\
\hline Q96RS0 & GIGLDESELDSEAELMR & Phospho(ST) \\
\hline Q9BXP5 & ERFSPPR & Phospho(ST) \\
\hline Q9BXP5 & HELSPPQKR & Phospho(ST) \\
\hline Q9BXP5 & TQLWASEPGTPPLPTSLPSQNPILK & Phospho(ST) \\
\hline P46937 & GDSETDLEALFNAVMNPK & Phospho(ST) \\
\hline P46937 & $\begin{array}{l}\text { AHSSPASLQLGAVSPGTLTPTGVVSGPAAT } \\
\text { PTAQHLR }\end{array}$ & Phospho(ST) \\
\hline Q96PU5 & SLSSPTVTLSAPLEGAK & Phospho(ST) \\
\hline P28290 & MPSPFR & Phospho(ST) \\
\hline P28290 & SLASIEAK & Phospho(ST) \\
\hline P28290 & ASVALTPTAPSR & Phospho(ST) \\
\hline Q16555 & GLYDGPVCEVSVTPK & Phospho(ST) \\
\hline Q99590 & SRSHSRSPSR & 2 Phospho(ST) \\
\hline Q99590 & SSSNDSVDEETAESDTSPVLEK & Phospho(ST) \\
\hline Q99590 & $\begin{array}{l}\text { AETASQSQRSPISDNSGCDAPGNSNPSLSVP } \\
\text { SSAESEK }\end{array}$ & Phospho(ST) \\
\hline Q8TAQ2 & KRSPSPSPTPEAK & 2 Phospho(ST) \\
\hline Q8TAQ2 & DMDEPSPVPNVEEVTLPK & Phospho(ST) \\
\hline P38432 & LGFSLTPSK & Phospho(ST) \\
\hline P38432 & AFQLEEGEETEPDCK & Phospho(ST) \\
\hline Q9UEW8 & TEDGDWEWSDDEMDEK & Phospho(ST) \\
\hline Q01831 & VIKDEALSDGDDLR & Phospho(ST) \\
\hline Q01831 & $\begin{array}{l}\text { SEAAAPHTDAGGGLSSDEEEGTSSQAEAA } \\
\mathrm{R}\end{array}$ & 2 Phospho(ST) \\
\hline P22466 & LLDLPAAASSEDIERS & Phospho(ST) \\
\hline Q9UMZ2 & QLSLEGSGLGVEDLK & Phospho(ST) \\
\hline P31943 & HTGPNSPDTANDGFVR & Phospho(ST) \\
\hline P98175 & LASDDRPSPPR & Phospho(ST) \\
\hline P98175 & HRHSPTGPPGFPR & Phospho(ST) \\
\hline P98175 & GLVAAYSGESDSEEEQER & 3 Phospho(ST) \\
\hline Q15019 & IYHLPDAESDEDEDFK & Phospho(ST) \\
\hline Q15019 & IYHLPDAESDEDEDFKEQTR & Phospho(ST) \\
\hline Q9UJX5 & IKEEVLSESEAENQQAGAAALAPEIVIK & Phospho(ST) \\
\hline P12270 & EGVQGPLNVSLSEEGK & Phospho(ST) \\
\hline Q53F19 & AEAPAGPALGLPSPEAESGVDR & Phospho(ST) \\
\hline Q9Y5S9 & MREDYDSVEQDGDEPGPQR & Phospho(ST) \\
\hline Q9Y608 & RGSGDTSSLIDPDTSLSELR & Phospho(ST) \\
\hline Q9UNS2 & SMGSQEDDSGNKPSSYS & Phospho(ST) \\
\hline Q99569 & VASPSQGQVGSSSPK & Phospho(ST) \\
\hline
\end{tabular}




\begin{tabular}{|c|c|c|}
\hline Protein & Peptide sequence & Variable modification \\
\hline Q99569 & DGWNQNHFITPVSTLER & Phospho(ST) \\
\hline O14950 & ATSNVFAMFDQSQIQEFK & Phospho(ST) \\
\hline \multirow[t]{2}{*}{ O14950 } & ATSNVFAMFDQSQIQEFK & Oxidation(M); \\
\hline & & Phospho(ST) \\
\hline \multirow[t]{2}{*}{ O14950 } & ATSNVFAMFDQSQIQEFK & Oxidation(M); \\
\hline & & 2 Phospho(ST) \\
\hline Q5XUX1 & TWDDDSDPESETDPDAQAK & Phospho(ST) \\
\hline Q8N3X1 & VQTTPKVEEEQDLK & Phospho(ST) \\
\hline Q8N3X1 & IDENSDKEMEVEESPEK & 2 Phospho(ST) \\
\hline Q8N3X1 & ATGGLCLLGAYADSDDDDNDVSEK & Phospho(ST) \\
\hline Q66K74 & LSLSPLR & Phospho(ST) \\
\hline Q66K74 & AVPMAPAPASPGSSNDSSAR & Phospho(ST) \\
\hline Q66K74 & ALELPLAASSIPRPRTPSPESHR & 2 Phospho(ST) \\
\hline P78344 & TQTPPLGQTPQLGLK & Phospho(ST) \\
\hline O43432 & SPVPAQIAITVPK & Phospho(ST) \\
\hline O43432 & RSPVPAQIAITVPK & Phospho(ST) \\
\hline Q9UNX4 & GSSPGIQDTLEAEDGAFETDEAPEDR & Phospho(ST) \\
\hline P31350 & VPLAPITDPQQLQLSPLK & Phospho(ST) \\
\hline \multirow[t]{2}{*}{ O00567 } & EELMSSDLEETAGSTSIPK & Oxidation(M); \\
\hline & & Phospho(ST) \\
\hline O00567 & SFSKEELMSSDLEETAGSTSIPK & Phospho(ST) \\
\hline \multirow[t]{2}{*}{ O00567 } & SFSKEELMSSDLEETAGSTSIPK & Oxidation(M); \\
\hline & & Phospho(ST) \\
\hline Q86VM9 & LGVSVSPSR & Phospho(ST) \\
\hline P40222 & RPEGPGAQAPSSPR & Phospho(ST) \\
\hline \multirow[t]{2}{*}{ P40222 } & SSPGQPEAGPEGAQERPSQAAPAVEAEGPG & Phospho(ST) \\
\hline & SSQAPR & \\
\hline O14745 & SASSDTSEELNSQDSPPK & Phospho(ST) \\
\hline Q7Z422 & SKSPPKVPIVIQDDSLPAGPPPQIR & Phospho(ST) \\
\hline Q7Z422 & SKSPPKVPIVIQDDSLPAGPPPQIR & 2 Phospho(ST) \\
\hline P11717 & LVSFHDDSDEDLLHI & Phospho(ST) \\
\hline P11717 & ALSSLHGDDQDSEDEVLTIPEVK & Phospho(ST) \\
\hline P51608 & AETSEGSGSAPAVPEASASPK & Phospho(ST) \\
\hline Q9HCN4 & GTLDEEDEEADSDTDDIDHR & Phospho(ST) \\
\hline Q9UHB6 & TVSPPIR & Phospho(ST) \\
\hline Q9UHB6 & ETPHSPGVEDAPIAK & Phospho(ST) \\
\hline Q9UHB6 & SEVQQPVHPKPLSPDSR & Phospho(ST) \\
\hline Q96QR8 & RGGGSGGGEESEGEEVDED & Phospho(ST) \\
\hline \multirow[t]{2}{*}{ Q96QR8 } & DSLGDFIEHYAQLGPSSPEQLAAGAEEGGG & Phospho(ST) \\
\hline & $\mathrm{PR}$ & \\
\hline Q7Z2W4 & TVFSPTLPAAR & Phospho(ST) \\
\hline Q8ND56 & SSPQLDPLR & Phospho(ST) \\
\hline Q8ND56 & RSPVSTRPLPSASQK & Phospho(ST) \\
\hline
\end{tabular}




\begin{tabular}{|c|c|c|}
\hline Protein & Peptide sequence & Variable modification \\
\hline Q8ND56 & SPTMEQAVQTASAHLPAPAAVGR & Phospho(ST) \\
\hline Q8ND56 & KSPTMEQAVQTASAHLPAPAAVGR & Phospho(ST) \\
\hline $\mathrm{O} 15042$ & SSHKDSPR & Phospho(ST) \\
\hline O15042 & SRSSHKDSPR & 2 Phospho(ST) \\
\hline P23193 & KEPAITSQNSPEAR & Phospho(ST) \\
\hline P23193 & KKEPAITSQNSPEAR & Phospho(ST) \\
\hline Q9H4G0 & GAPSQDDESGGIEDSPDR & Phospho(ST) \\
\hline Q6L8Q7 & $\begin{array}{l}\text { EAKPGAAEPEVGVPSSLSPSSPSSSWTETDV } \\
\text { EER }\end{array}$ & Phospho(ST) \\
\hline Q5HYJ3 & ISNLSPEEEQGLWK & Phospho(ST) \\
\hline P20042 & IESDVQEPTEPEDDLDIMLGNK & Phospho(ST) \\
\hline Q8WWQ0 & TAFYNEDDSEEEQR & Phospho(ST) \\
\hline Q9P289 & LADFGVAGQLTDTQIK & Phospho(ST) \\
\hline P08621 & YDERPGPSPLPHR & Phospho(ST) \\
\hline Q9NQG5 & LSMEDSKSPPPK & Phospho(ST) \\
\hline P52701 & VHVQFFDDSPTR & Phospho(ST) \\
\hline P52701 & SEEDNEIESEEEVQPK & Phospho(ST) \\
\hline Q7Z5L9 & KPSPEPEGEVGPPK & Phospho(ST) \\
\hline Q7Z5L9 & RKPSPEPEGEVGPPK & Phospho(ST) \\
\hline Q7Z5L9 & RPASVSSSAAVEHEQR & Phospho(ST) \\
\hline P30414 & LDTPDINIVLK & Phospho(ST) \\
\hline Q7Z5K2 & VEEESTGDPFGFDSDDESLPVSSK & Phospho(ST) \\
\hline Q5JTV8 & LQQQHSEQPPLQPSPVMTR & Phospho(ST) \\
\hline Q5JTV8 & GLRDSHSSEEDEASSQTDLSQTISK & 3 Phospho(ST) \\
\hline Q14980 & QSMAFSILNTPKK & $\begin{array}{l}\text { Oxidation(M); } \\
\text { Phospho(ST) }\end{array}$ \\
\hline Q14980 & VSLEPHQGPGTPESK & Phospho(ST) \\
\hline Q14980 & TQPDGTSVPGEPASPISQR & Phospho(ST) \\
\hline Q9UN86 & STTPPPAEPVSLPQEPPK & Phospho(ST) \\
\hline Q9UN86 & YEDEVFGDSEPELDEESEDEVEEEQEER & Phospho(ST) \\
\hline Q9UN86 & YEDEVFGDSEPELDEESEDEVEEEQEER & 2 Phospho(ST) \\
\hline P17812 & SGSSSPDSEITELK & Phospho(ST) \\
\hline P17812 & SGSSSPDSEITELK & 2 Phospho(ST) \\
\hline P17812 & SGSSSPDSEITELKFPSINHD & Phospho(ST) \\
\hline P17812 & DTYSDRSGSSSPDSEITELK & Phospho(ST); Phospho(Y) \\
\hline P08559 & YGMGTSVER & Phospho(ST) \\
\hline P08559 & YGMGTSVER & $\begin{array}{l}\text { Oxidation(M); } \\
\text { Phospho(ST) }\end{array}$ \\
\hline P08559 & YHGHSMSDPGVSYR & Phospho(ST) \\
\hline P08559 & YHGHSMSDPGVSYR & Phospho(ST); Phospho(Y) \\
\hline P29803 & YHGHSMSDPGVSYR & Phospho(ST) \\
\hline P29803 & YHGHSMSDPGVSYR & Phospho(ST); Phospho(Y) \\
\hline O75153 & SEDPPGQEAGSEEEGSSASGLAK & Phospho(ST) \\
\hline
\end{tabular}




\begin{tabular}{|c|c|c|}
\hline Protein & Peptide sequence & Variable modification \\
\hline Q7Z4H7 & AVLSDSPQLSEGK & Phospho(ST) \\
\hline P35269 & LDTGPQSLSGK & Phospho(ST) \\
\hline P35269 & GNSRPGTPSAEGGSTSSTLR & 2 Phospho(ST) \\
\hline Q9UKN8 & ILLVDSPGMGNADDEQQEEGTSSK & Phospho(ST) \\
\hline Q96B36 & SLPVSVPVWGFK & Phospho(ST) \\
\hline Q96B36 & AATAARPPAPPPAPQPPSPTPSPPRPTLAR & 2 Phospho(ST) \\
\hline Q9NXG2 & FTDKDQQPSGSEGEDDDAEAALK & 2 Phospho(ST) \\
\hline Q9NXG2 & FTDKDQQPSGSEGEDDDAEAALKK & 2 Phospho(ST) \\
\hline Q15276 & AQSTDSLGTSGSLQSK & Phospho(ST) \\
\hline Q9NWW5 & HGSVSADEAAR & Phospho(ST) \\
\hline Q5VT52 & SFNYSPNSSTSEVSSTSASK & Phospho(ST) \\
\hline Q6P2E9 & DSQDASAEQSDHDDEVASLASASGGFGTK & Phospho(ST) \\
\hline Q6P2E9 & TRSPDVISSASTALSQDIPEIASEALSR & Phospho(ST) \\
\hline Q8IVT5 & ALHSFITPPTTPQLR & Phospho(ST) \\
\hline Q8IVT5 & RTESVPSDINNPVDR & Phospho(ST) \\
\hline Q8IVT5 & $\begin{array}{l}\text { ADVLEAHEAEAEEPEAGKSEAEDDEDEVD } \\
\text { DLPSSR }\end{array}$ & Phospho(ST) \\
\hline P62750 & IRTSPTFR & Phospho(ST) \\
\hline Q05519 & DYDEEEQGYDSEKEK & Phospho(ST) \\
\hline Q5VSL9 & AASPPASASDLIEQQQK & Phospho(ST) \\
\hline Q02790 & SNTAGSQSQVETEA & Phospho(ST) \\
\hline P17275 & DATPPVSPINMEDQER & 2 Phospho(ST) \\
\hline P17275 & SRDATPPVSPINMEDQER & 3 Phospho(ST) \\
\hline Q13895 & MPQDGSDDEDEEWPTLEK & Phospho(ST) \\
\hline Q9P206 & KPSVGVPPPASPSYPR & Phospho(ST) \\
\hline O96028 & IQDPTEDAEAEDTPR & Phospho(ST) \\
\hline O43237 & DFQDYMEPEEGCQGSPQR & Phospho(ST) \\
\hline Q8N163 & SVASNQSEMEFSSLQDMPK & 3 Phospho(ST) \\
\hline Q9UK58 & AEEKSPISINVK & Phospho(ST) \\
\hline Q9UK58 & GLNPDGTPALSTLGGFSPASKPSSPR & 3 Phospho(ST) \\
\hline Q4KMP7 & AAGGAPSPPPPVR & Phospho(ST) \\
\hline Q4KMP7 & QQPPLGPSSSLLSLPGLK & 2 Phospho(ST) \\
\hline Q4KMP7 & TEEARPSPAPGPGTPTGTPTR & Phospho(ST) \\
\hline P85037 & SGGLQTPECLSR & 2 Phospho(ST) \\
\hline P85037 & EGSPIPHDPEFGSK & Phospho(ST) \\
\hline P85037 & SAPASPTHPGLMSPR & 2 Phospho(ST) \\
\hline P06493 & IGEGTYGVVYK & Phospho(Y) \\
\hline P06493 & IGEGTYGVVYK & Phospho(ST) \\
\hline P24941 & IGEGTYGVVYK & Phospho(Y) \\
\hline P24941 & IGEGTYGVVYK & Phospho(ST) \\
\hline Q05655 & ASTFCGTPDYIAPEILQGLK & Phospho(ST) \\
\hline Q7KZI7 & SRNSPLLER & Phospho(ST) \\
\hline Q7KZI7 & VPASPLPGLER & Phospho(ST) \\
\hline
\end{tabular}




\begin{tabular}{|c|c|c|}
\hline Protein & Peptide sequence & Variable modification \\
\hline Q9Y6E2 & FVEWLQNAEEESESEGEEN & 2 Phospho(ST) \\
\hline Q8TC07 & NDSPTQIPVSSDVCR & Phospho(ST) \\
\hline Q16512 & LNLGTDSDSSPQK & Phospho(ST) \\
\hline Q16512 & TDVSNFDEEFTGEAPTLSPPR & Phospho(ST) \\
\hline Q14244 & LFVTPPEGSSR & Phospho(ST) \\
\hline Q14244 & LQLSPWESSVVNR & Phospho(ST) \\
\hline O15173 & LLKPGEEPSEYTDEEDTK & Phospho(ST) \\
\hline O15173 & LLKPGEEPSEYTDEEDTKDHNK & Phospho(ST) \\
\hline O15173 & LLKPGEEPSEYTDEEDTKDHNKQD & Phospho(ST) \\
\hline Q15021 & YQPLASTASDNDFVTPEPR & Phospho(ST) \\
\hline Q9NW82 & TMFAQVESDDEEAK & Phospho(ST) \\
\hline Q13247 & SNSPLPVPPSK & Phospho(ST) \\
\hline O60231 & LLEDSEESSEETVSR & 2 Phospho(ST) \\
\hline Q9NQ55 & VGGSDEEASGIPSR & Phospho(ST) \\
\hline O00559 & KLSGDQITLPTTVDYSSVPK & Phospho(ST) \\
\hline Q99958 & SEAASPALPVITK & Phospho(ST) \\
\hline P51114 & RGPNYTSGYGTNSELSNPSETESER & Phospho(ST) \\
\hline Q14694 & NHSVNEEEQEEQGEGSEDEWEQVGPR & Phospho(ST) \\
\hline Q14847 & GFSVVADTPELQR & Phospho(ST) \\
\hline Q08499 & LMHSSSLTNSSIPR & Phospho(ST) \\
\hline Q08499 & EWYQSTIPQSPSPAPDDPEEGR & Phospho(ST) \\
\hline Q9H7F0 & LVHDSLEDLQMTR & Phospho(ST) \\
\hline Q86U90 & LFRPPSPAPAAPGAR & Phospho(ST) \\
\hline Q0JRZ9 & VSIGNITLSPAISR & Phospho(ST) \\
\hline Q9BVG4 & GADSGEEKEEGINR & Phospho(ST) \\
\hline Q9UBC2 & TVFPGAVPVLPASPPPK & Phospho(ST) \\
\hline Q9UBC2 & STPSHGSVSSLNSTGSLSPK & Phospho(ST) \\
\hline O94888 & SESLIDASEDSQLEAAIR & Phospho(ST) \\
\hline Q15477 & ASSLEDLVLK & Phospho(ST) \\
\hline Q13442 & SLDSDESEDEEDDYQQK & 2 Phospho(ST) \\
\hline Q6AI08 & APAGPSLEETSVSSPK & Phospho(ST) \\
\hline O75376 & AQLSPGIYDDTSAR & Phospho(ST) \\
\hline Q92793 & SISPSALQDLLR & Phospho(ST) \\
\hline Q96JM3 & LAPVPSPEPQKPAPVSPESVK & Phospho(ST) \\
\hline Q96JM3 & GQESSSDQEQVDVESIDFSK & 2 Phospho(ST) \\
\hline Q8IXM2 & KVYEDSGIPLPAESPK & Phospho(ST) \\
\hline P17029 & EATGLSPQAAQEK & Phospho(ST) \\
\hline Q9H2Y7 & SLSESSVIMDR & Phospho(ST) \\
\hline Q16513 & ASSLGEIDESSELR & Phospho(ST) \\
\hline Q16513 & GREDVSNFDDEFTSEAPILTPPREPR & Phospho(ST) \\
\hline Q9NZ63 & VGDTEKPEPERSPPNR & Phospho(ST) \\
\hline Q9NZ63 & RGDSESEEDEQDSEEVR & 2 Phospho(ST) \\
\hline P26038 & DKYKTLR & Phospho(ST) \\
\hline
\end{tabular}




\begin{tabular}{|c|c|c|c|}
\hline Protein & Peptide sequence & Variable modification & \\
\hline \multirow[t]{2}{*}{ O75694 } & PSSLLGAAMPASTSAAALQEALENAGR & Oxidation(M); & \\
\hline & & Phospho(ST) & \\
\hline P49959 & GVDFESSEDDDDDPFMNTSSLR & 2 Phospho(ST) & \\
\hline \multirow[t]{2}{*}{ P49959 } & SQSEESASAFSADDLMSIDLAEQMANDSD & Oxidation(M); & 4 \\
\hline & DSISAATNKGRGR & Phospho(ST) & \\
\hline Q9Y314 & VLPSFWIPSLTPEAK & Phospho(ST) & \\
\hline Q3MHD2 & TETPPPLASLNVSK & Phospho(ST) & \\
\hline Q13557 & ESTESSNTTIEDEDVK & Phospho(ST) & \\
\hline Q9Y2V2 & TFSATVR & Phospho(ST) & \\
\hline Q9Y2V2 & GNVVPSPLPTR & Phospho(ST) & \\
\hline P62753 & RLSSLR & Phospho(ST) & \\
\hline Q9Y253 & RLSSLR & Phospho(ST) & \\
\hline Q15459 & FGESEEVEMEVESDEEDDKQEK & Phospho(ST) & \\
\hline \multirow[t]{2}{*}{ Q15459 } & FGESEEVEMEVESDEEDDKQEK & Oxidation(M); & \\
\hline & & Phospho(ST) & \\
\hline Q15459 & AEEPPSQLDQDTQVQDMDEGSDDEEEGQK & Phospho(ST) & \\
\hline \multirow[t]{2}{*}{ Q15459 } & AEEPPSQLDQDTQVQDMDEGSDDEEEGQK & Oxidation(M); & \\
\hline & & Phospho(ST) & \\
\hline P08708 & LLDFGSLSNLQVTQPTVGMNFK & Phospho(ST) & \\
\hline \multirow[t]{2}{*}{ Q9NWH9 } & SPGHMVILDQTK & Oxidation(M); & \\
\hline & & Phospho(ST) & \\
\hline Q9NWH9 & DGQDAIAQSPEKESK & Phospho(ST) & \\
\hline Q9NWH9 & AGAGMITQHSSNASPINR & Phospho(ST) & \\
\hline Q9NWH9 & ISSKSPGHMVILDQTK & 2 Phospho(ST) & \\
\hline Q6Y7W6 & SQSWEER & Phospho(ST) & \\
\hline \multirow[t]{2}{*}{ Q6Y7W6 } & VGVEASEETPQTSSSSARPGTPSDHQSQEA & Phospho(ST) & \\
\hline & SQFER & & \\
\hline Q14978 & RASSPFR & Phospho(ST) & \\
\hline Q14978 & VADNSFDAK & Phospho(ST) & \\
\hline Q14978 & LQTPNTFPK & Phospho(ST) & \\
\hline Q14978 & GKGSPRPQAPK & Phospho(ST) & \\
\hline Q14978 & AAKNSEEEEEEK & Phospho(ST) & \\
\hline Q14978 & AAKNSEEEEEEKK & Phospho(ST) & \\
\hline P30419 & GGLSPANDTGAK & Phospho(ST) & \\
\hline P29375 & DLDLEPLSDLEEGLEETR & Phospho(ST) & \\
\hline Q9HCH0 & SPSPGGPQLSPQLPR & Phospho(ST) & \\
\hline P78316 & HNDIVDSDSDAEDR & 2 Phospho(ST) & \\
\hline Q15554 & DLVLPTQALPASPALK & Phospho(ST) & \\
\hline Q6SPF0 & AAAAAATAPPSPGPAQPGPR & Phospho(ST) & \\
\hline $\mathrm{O} 43823$ & SGDEEFKGEDELCDSGR & Phospho(ST) & \\
\hline O95365 & GGAPDPSPGATATPGAPAQPSSPDAR & Phospho(ST) & \\
\hline Q9HD67 & TSFLEGTLR & Phospho(ST) & \\
\hline Q9HD67 & FDTDDELSYR & Phospho(ST) & \\
\hline
\end{tabular}




\begin{tabular}{|c|c|c|}
\hline Protein & Peptide sequence & Variable modification \\
\hline P21359 & GSEGYLAATYPTVGQTSPR & Phospho(ST) \\
\hline Q7Z4S6 & EKELSPPPGLPSK & Phospho(ST) \\
\hline Q7Z4S6 & KLSSSDAPAQDTGSSAAAVETDASR & Phospho(ST) \\
\hline Q03111 & RSPESCSKPEK & Phospho(ST) \\
\hline Q03111 & RPATADSPKPSAK & Phospho(ST) \\
\hline P08651 & NWTEDMEGGISSPVKK & Phospho(ST) \\
\hline P08651 & TEMDKSPFNSPSPQDSPR & Phospho(ST) \\
\hline P08651 & TEMDKSPFNSPSPQDSPR & 2 Phospho(ST) \\
\hline P08651 & KTEMDKSPFNSPSPQDSPR & 2 Phospho(ST) \\
\hline Q9BXB4 & NSSCGGGISSSSSSR & Phospho(ST) \\
\hline Q9BXB4 & SFSLASSSNSPISQR & Phospho(ST) \\
\hline P23396 & DEILPTTPISEQK & Phospho(ST) \\
\hline P25490 & EEVVGGDDSDGLR & Phospho(ST) \\
\hline P25490 & DIDHETVVEEQIIGENSPPDYSEYMTGK & Phospho(ST) \\
\hline Q9UH62 & YNDWSDDDDDSNESK & Phospho(ST) \\
\hline \multirow[t]{2}{*}{ Q86SQ0 } & SGAASMPSSPK & Oxidation(M); \\
\hline & & Phospho(ST) \\
\hline Q86SQ0 & LQLSDEESVFEEALMSPDTR & Phospho(ST) \\
\hline Q07955 & VDGPRSPSYGR & Phospho(ST) \\
\hline Q07955 & VDGPRSPSYGR & Phospho(ST); Phospho(Y) \\
\hline Q07955 & VKVDGPRSPSYGR & 2 Phospho(ST) \\
\hline Q8N6N3 & LLPEGEETLESDDEKDEHTSK & Phospho(ST) \\
\hline Q96PC5 & AFLSPPTLLEGPLR & Phospho(ST) \\
\hline Q99733 & EFITGDVEPTDAESEWHSENEEEEK & Phospho(ST) \\
\hline Q99733 & REFITGDVEPTDAESEWHSENEEEEK & Phospho(ST) \\
\hline Q92685 & SGSAAQAEGLCK & Phospho(ST) \\
\hline Q99442 & EELEQQTDGDCEEDEEEENDGETPK & Phospho(ST) \\
\hline Q9H6Z4 & VLSPPKLNEVSSDANR & Phospho(ST) \\
\hline Q9H6Z4 & SAGGSSPEGGEDSDREDGNYCPPVKR & 3 Phospho(ST) \\
\hline P27708 & IHRASDPGLPAEEPK & Phospho(ST) \\
\hline P36871 & AIGGIILTASHNPGGPNGDFGIK & Phospho(ST) \\
\hline P18206 & SFLDSGYR & Phospho(Y) \\
\hline P18206 & SLLDASEEAIKK & Phospho(ST) \\
\hline Q6IQ22 & AGGGGGLGAGSPALSGGQGR & Phospho(ST) \\
\hline P49023 & FIHQQPQSSSPVYGSSAK & Phospho(ST) \\
\hline P49023 & TGSSSPPGGPPKPGSQLDSMLGSLQSDLNK & Phospho(ST) \\
\hline \multirow[t]{2}{*}{ P49023 } & TGSSSPPGGPPKPGSQLDSMLGSLQSDLNK & Oxidation(M); \\
\hline & & Phospho(ST) \\
\hline O60256 & LGIAVIHGEAQDAESDLVDGRHSPPMVR & Phospho(ST) \\
\hline \multirow[t]{2}{*}{ O60256 } & LGIAVIHGEAQDAESDLVDGRHSPPMVR & Oxidation(M); \\
\hline & & Phospho(ST) \\
\hline \multirow[t]{2}{*}{ Q9P0K7 } & DRLSDSTTGADSLLDISSEADQQDLLSLLQ & Phospho(ST) \\
\hline & AK & \\
\hline
\end{tabular}




\begin{tabular}{|c|c|c|}
\hline Protein & Peptide sequence & Variable modification \\
\hline Q13459 & VQEKPDSPGGSTQIQR & Phospho(ST) \\
\hline \multirow[t]{2}{*}{ Q14677 } & TIDLGAAAHYTGDKASPDQNASTHTPQSS & Phospho(ST) \\
\hline & $\mathrm{VK}$ & \\
\hline O94979 & AQGEPVAGHESPK & Phospho(ST) \\
\hline O94979 & AQGEPVAGHESPKIPYEK & Phospho(ST) \\
\hline O94979 & DSDQVAQSDGEESPAAEEQLLGEHIK & Phospho(ST) \\
\hline O94979 & DSDQVAQSDGEESPAAEEQLLGEHIKEEK & Phospho(ST) \\
\hline Q01581 & RPTPNDDTLDEGVGLVHSNIATEHIPSPAK & Phospho(ST) \\
\hline Q7L014 & AALGLQDSDDEDAAVDIDEQIESMFNSK & Phospho(ST) \\
\hline \multirow[t]{2}{*}{ Q7L014 } & AALGLQDSDDEDAAVDIDEQIESMFNSK & Oxidation(M); \\
\hline & & Phospho(ST) \\
\hline Q01167 & EGSPAPLEPEPGAAQPK & Phospho(ST) \\
\hline Q01167 & FAQSAPGSPLSSQPVLITVQR & Phospho(ST) \\
\hline Q4G0J3 & SRPTSEGSDIESTEPQK & Phospho(ST) \\
\hline Q4G0J3 & SRPTSEGSDIESTEPQK & 2 Phospho(ST) \\
\hline O76080 & MSPMGTASGSNSPTSDSASVQR & Phospho(ST) \\
\hline Q9C0C9 & LIHGEDSDSEGEEEGR & 2 Phospho(ST) \\
\hline Q9C0C9 & NMTVEQLLTGSPTSPTVEPEKPTR & Phospho(ST) \\
\hline Q8IZL8 & GSPDGSLQTGKPSAPK & Phospho(ST) \\
\hline Q27J81 & DPTSLLGVLQAEADSTSEGLEDAVHSR & Phospho(ST) \\
\hline \multirow[t]{2}{*}{ Q13136 } & VTSSMSSPSMQPK & 2 Oxidation(M); \\
\hline & & Phospho(ST) \\
\hline Q92538 & ADAPDAGAQSDSELPSYHQNDVSLDR & Phospho(ST) \\
\hline Q13595 & RSPSPYYSR & 2 Phospho(ST) \\
\hline Q13595 & RRSPSPYYSR & Phospho(ST); Phospho(Y) \\
\hline Q13595 & RRSPSPYYSR & 2 Phospho(ST) \\
\hline Q8TCJ2 & ENPPVEDSSDEDDKR & 2 Phospho(ST) \\
\hline Q86VR2 & GQTPLTEGSEDLDGHSDPEESFAR & Phospho(ST) \\
\hline Q86VR2 & AMDNHSDSEEELAAFCPQLDDSTVAR & 2 Phospho(ST) \\
\hline Q96CB8 & LEKPETQSSPITVQSSK & Phospho(ST) \\
\hline Q9H7N4 & QRSPSPAPAPAPAAAAGPPTR & 2 Phospho(ST) \\
\hline Q9H7N4 & FDIYDPFHPTDEAYSPPPAPEQK & Phospho(ST) \\
\hline Q969E4 & GTDDSPKDSQEDLQER & Phospho(ST) \\
\hline P19484 & VHGLPTTSPSGMNMAELAQQVVK & Phospho(ST) \\
\hline Q6UN15 & AEFTSPPSLFK & Phospho(ST) \\
\hline Q6UN15 & DHSPTPSVFNSDEER & 2 Phospho(ST) \\
\hline Q6UN15 & VTETEDDSDSDSDDDEDDVHVTIGDIK & 3 Phospho(ST) \\
\hline Q14157 & YPSSISSSPQK & Phospho(ST) \\
\hline Q14157 & RYPSSISSSPQK & Phospho(ST) \\
\hline Q14157 & RYPSSISSSPQK & 2 Phospho(ST) \\
\hline Q14157 & STSAPQMSPGSSDNQSSSPQPAQQK & Phospho(ST) \\
\hline Q7Z3C6 & HPEPVPEEGSEDELPPQVHKV & Phospho(ST) \\
\hline Q7Z3C6 & LEASYSDSPPGEEDLLVHVAEGSK & Phospho(ST) \\
\hline
\end{tabular}




\begin{tabular}{|c|c|c|}
\hline Protein & Peptide sequence & Variable modification \\
\hline Q99961 & IAASSSFR & Phospho(ST) \\
\hline Q9H3Z4 & SLSTSGESLYHVLGLDK & Phospho(ST) \\
\hline Q14126 & VVPSFLPVDQGGSLVGR & Phospho(ST) \\
\hline Q14126 & $\begin{array}{l}\text { NVIATETSYVTGSTMPPTTVILGPSQPQSLI } \\
\text { VTER }\end{array}$ & $\begin{array}{l}4 \quad \text { Phospho(ST); } \\
\text { Phospho(Y) }\end{array}$ \\
\hline Q13185 & RKSLSDSESDDSK & Phospho(ST) \\
\hline P20020 & IEDSEPHIPLIDDTDAEDDAPTK & Phospho(ST) \\
\hline P51532 & GRPPAEKLSPNPPNLTK & Phospho(ST) \\
\hline P51532 & $\begin{array}{l}\text { KAENAEGQTPAIGPDGEPLDETSQMSDLPV } \\
\mathrm{K}\end{array}$ & Phospho(ST) \\
\hline Q86XP3 & $\begin{array}{l}\text { QQFHSKPVDSDSDDDPLEAFMAEVEDQAA } \\
\mathrm{R}\end{array}$ & 2 Phospho(ST) \\
\hline Q86XP3 & $\begin{array}{l}\text { YMAENPTAGVVQEEEEDNLEYDSDGNPIA } \\
\text { PTK }\end{array}$ & Phospho(Y) \\
\hline Q86XP3 & $\begin{array}{l}\text { YMAENPTAGVVQEEEEDNLEYDSDGNPIA } \\
\text { PTKK }\end{array}$ & Phospho(ST) \\
\hline Q8TEK3 & VAGPADAPMDSGAEEEK & Phospho(ST) \\
\hline O76021 & AAESETPGKSPEKKPK & 2 Phospho(ST) \\
\hline O76021 & ATNESEDEIPQLVPIGK & Phospho(ST) \\
\hline Q15637 & TGDLGIPPNPEDRSPSPEPIYNSEGK & 2 Phospho(ST) \\
\hline Q9UQ80 & ALLQSSASR & Phospho(ST) \\
\hline Q9UIG0 & LAEDEGDSEPEAVGQSR & Phospho(ST) \\
\hline Q9BWW4 & NSPNNISGISNPPGTPR & Phospho(ST) \\
\hline E9PAV3 & QVATSSSPK & Phospho(ST) \\
\hline E9PAV3 & $\begin{array}{l}\text { VQGEAVSNIQENTQTPTVQEESEEEEVDET } \\
\text { GVEVK }\end{array}$ & Phospho(ST) \\
\hline Q53EL6 & FVSEGDGGR & Phospho(ST) \\
\hline Q53EL6 & SGLTVPTSPK & Phospho(ST) \\
\hline Q9UJX2 & RVSPLNLSSVTP & Phospho(ST) \\
\hline Q93009 & AGEQQLSEPEDMEMEAGDTDDPPR & Phospho(ST) \\
\hline P56524 & QEPIESDEEEAEPPR & Phospho(ST) \\
\hline P38159 & GLPPSMER & Phospho(ST) \\
\hline Q5SZK8 & SRAGIAISAFNLK & Phospho(ST) \\
\hline Q9UQN3 & ATISDEEIER & Phospho(ST) \\
\hline Q9Y6R0 & $\begin{array}{l}\text { KAEAAAAPTVAPGPAQPGHVSPTPATTSPG } \\
\text { EK }\end{array}$ & Phospho(ST) \\
\hline Q6PID6 & SEAPAEVTHFSPK & Phospho(ST) \\
\hline P50552 & NSTTLPR & Phospho(ST) \\
\hline Q76FK4 & NSISDDDTDSEDELR & 3 Phospho(ST) \\
\hline Q76FK4 & LQDSSSEEEDVTEETDHR & 3 Phospho(ST) \\
\hline Q8WYP5 & CLVAGLLSPR & Phospho(ST) \\
\hline Q8WYP5 & NLSFNELYPSGTLK & Phospho(ST) \\
\hline P26641 & TPEFLR & Phospho(ST) \\
\hline
\end{tabular}




\begin{tabular}{|c|c|c|}
\hline Protein & Peptide sequence & Variable modification \\
\hline Q9BVJ6 & DYLLSESEDEGDNDGER & 2 Phospho(ST) \\
\hline Q92769 & IACDEEFSDSEDEGEGGRR & 2 Phospho(ST) \\
\hline Q92769 & $\begin{array}{l}\text { MLPHAPGVQMQAIPEDAVHEDSGDEDGED } \\
\text { PDKR }\end{array}$ & Phospho(ST) \\
\hline Q92769 & $\begin{array}{l}\text { MLPHAPGVQMQAIPEDAVHEDSGDEDGED } \\
\text { PDKR }\end{array}$ & $\begin{array}{l}\text { Oxidation(M); } \\
\text { Phospho(ST) }\end{array}$ \\
\hline P50548 & GEGPGEAGGPLTPR & Phospho(ST) \\
\hline Q8NEF9 & AVTIANSPSKPSEK & Phospho(ST) \\
\hline Q16643 & LSSPVLHR & Phospho(ST) \\
\hline Q16643 & MAPTPIPTRSPSDSSTASTPVAEQIER & $\begin{array}{l}\text { Oxidation(M); } \\
\text { Phospho(ST) }\end{array}$ \\
\hline Q9Y383 & AMLDQLMGTSR & Phospho(ST) \\
\hline O60341 & EMDESLANLSEDEYYSEEER & 2 Phospho(ST) \\
\hline Q8NDX6 & AGSPDVLR & Phospho(ST) \\
\hline Q9H0G5 & VIETPENDFK & Phospho(ST) \\
\hline Q9H0G5 & VEENPDADSDFDAKSSADDEIEETR & 3 Phospho(ST) \\
\hline Q14669 & SESPPAELPSLR & Phospho(ST) \\
\hline O95248 & LGLGTLSSSLSR & Phospho(ST) \\
\hline Q7Z3K3 & SLDSEPSVPSAAKPPSPEK & Phospho(ST) \\
\hline Q92766 & QVAGDAPVEQATAETASPVHR & Phospho(ST) \\
\hline P42684 & GGFFSSFMK & Phospho(ST) \\
\hline P42684 & VPVLISPTLK & Phospho(ST) \\
\hline Q04323 & YGGSVGSQPPPVAPEPGPVPSSPSQEPPTKR & Phospho(ST) \\
\hline O75925 & GILSLPHQASPVSR & Phospho(ST) \\
\hline O75167 & ASIANSDGPTAGSQTPPFK & Phospho(ST) \\
\hline Q9BPX3 & TLHCEGTEINSDDEQESK & Phospho(ST) \\
\hline Q9BPX3 & CQTAEADSESDHEVPEPESEMK & 2 Phospho(ST) \\
\hline P49756 & SREKSR & Phospho(ST) \\
\hline P49756 & LGASNSPGQPNSVK & Phospho(ST) \\
\hline O00429 & SKPIPIMPASPQK & Phospho(ST) \\
\hline P01106 & PLNVSFTNR & Phospho(ST) \\
\hline P53999 & EQISDIDDAVR & Phospho(ST) \\
\hline Q8N122 & VLDTSSLTQSAPASPTNK & Phospho(ST) \\
\hline O75379 & NLLEDDSDEEEDFFLR & Phospho(ST) \\
\hline Q8TDD1 & LGPGRPLPTFPTSECTSDVEPDTR & Phospho(ST) \\
\hline O94875 & RKSEPAVGPPR & Phospho(ST) \\
\hline O94875 & TSPGRVDLPGSSTTLTK & Phospho(ST) \\
\hline Q9BZZ5 & RASEDTTSGSPPKK & Phospho(ST) \\
\hline Q9Y2U5 & DRSSPPPGYIPDELHQVAR & Phospho(ST) \\
\hline Q9UBB9 & GAAEEAELEDSDDEEKPVK & Phospho(ST) \\
\hline Q9UBB9 & TTQSMQDFPVVDSEEEAEEEFQK & Phospho(ST) \\
\hline Q02880 & KASGSENEGDYNPGR & 2 Phospho(ST) \\
\hline Q02880 & KTSFDQDSDVDIFPSDFPTEPPSLPR & Phospho(ST) \\
\hline
\end{tabular}




\begin{tabular}{|c|c|c|}
\hline Protein & Peptide sequence & Variable modification \\
\hline Q02880 & $\begin{array}{l}\text { VKASPITNDGEDEFVPSDGLDKDEYTFSPG } \\
\mathrm{K}\end{array}$ & 3 Phospho(ST) \\
\hline Q9P2N5 & KYPSPQK & Phospho(ST) \\
\hline O75400 & QETVADFTPK & Phospho(ST) \\
\hline O75400 & HKSDSPESDAER & 2 Phospho(ST) \\
\hline O75400 & DSGNWDTSGSELSEGELEK & 4 Phospho(ST) \\
\hline O75400 & DSGNWDTSGSELSEGELEKR & 4 Phospho(ST) \\
\hline Q9UKS6 & GGRSPDEVTLTSIVPTR & Phospho(ST) \\
\hline Q9UKS6 & DGTAPPPQSPGSPGTGQDEEWSDEESPR & Phospho(ST) \\
\hline P30622 & $\begin{array}{l}\text { ATSPLCTSTASMVSSSPSTPSNIPQKPSQPA } \\
\text { AK }\end{array}$ & Phospho(ST) \\
\hline Q6VY07 & TDLQGSASPSKVEGVHTPR & Phospho(ST) \\
\hline Q6NZI2 & LPAKLSISK & Phospho(ST) \\
\hline Q6NZI2 & $\begin{array}{l}\text { EGEELGEGERPEEDAAALELSSDEAVEVEE } \\
\text { VIEESR }\end{array}$ & 2 Phospho(ST) \\
\hline Q92508 & SGSEEAVTDPGER & Phospho(ST) \\
\hline P08648 & LLESSLSSSEGEEPVEYK & Phospho(ST) \\
\hline Q96IF1 & GAQPGRHSVTGYGDCAVGAR & Phospho(ST) \\
\hline Q9NPQ8 & GLMAGGRPEGQYSEDEDTDTDEYK & 2 Phospho(ST) \\
\hline O95239 & TFSLTEVR & Phospho(ST) \\
\hline Q92922 & KHSPSPPPPTPTESR & 2 Phospho(ST) \\
\hline O60832 & AGLESGAEPGDGDSDTTKK & Phospho(ST) \\
\hline O60832 & KRESESESDETPPAAPQLIK & 2 Phospho(ST) \\
\hline O95251 & SQQQPTPVTPK & Phospho(ST) \\
\hline O95466 & GEPPAPKSPPK & Phospho(ST) \\
\hline P53985 & EEETSIDVAGKPNEVTK & Phospho(ST) \\
\hline P53985 & KESKEEETSIDVAGKPNEVTK & Phospho(ST) \\
\hline O75970 & GSLPQLVSPIVSR & Phospho(ST) \\
\hline P26358 & EADDDEEVDDNIPEMPSPK & Phospho(ST) \\
\hline P26358 & EADDDEEVDDNIPEMPSPK & $\begin{array}{l}\text { Oxidation(M); } \\
\text { Phospho(ST) }\end{array}$ \\
\hline P48681 & ENQEPLRSPEVGDEEALRPLTK & Phospho(ST) \\
\hline P48681 & QEASTGQSPEDHASLAPPLSPDHSSLEAK & Phospho(ST) \\
\hline Q5VZL5 & VTQHESDNENEIQIQNK & Phospho(ST) \\
\hline Q12802 & TFSYIK & Phospho(ST) \\
\hline Q12802 & $\begin{array}{l}\text { SFSLEGLTGGAGVGNKPSSSLEVSSANAEE } \\
\text { LR }\end{array}$ & Phospho(ST) \\
\hline Q92733 & KPSDGSPDTKPSR & Phospho(ST) \\
\hline Q92733 & IAAPELHKGDSDSEEDEPTK & 2 Phospho(ST) \\
\hline O95453 & NNSFTAPSTVGK & Phospho(ST) \\
\hline Q9UPT8 & $\begin{array}{l}\text { AAKPGPAEAPSPTASPSGDASPPATAPYDP } \\
\mathrm{R}\end{array}$ & Phospho(ST) \\
\hline Q9Y232 & TAVDGFQSESPEKLDPVEQGQEDTVAPEV & Phospho(ST) \\
\hline
\end{tabular}




\begin{tabular}{|c|c|c|}
\hline Protein & Peptide sequence & Variable modification \\
\hline & AAEKPVGALLGPGAER & \\
\hline Q8NHV4 & LVTSGAESGNLNTSPSSNQTR & Phospho(ST) \\
\hline Q8IZ21 & SSSPVQVEEEPVR & Phospho(ST) \\
\hline Q96S55 & RPAAAAAAGSASPR & Phospho(ST) \\
\hline Q05209 & NLSFEIK & Phospho(ST) \\
\hline Q05209 & DVDVSEDSPPPLPER & Phospho(ST) \\
\hline Q09472 & TEIKEEEDQPSTSATQSSPAPGQSK & Phospho(ST) \\
\hline Q15059 & SESPPPLSDPK & Phospho(ST) \\
\hline Q92785 & ILEPDDFLDDLDDEDYEEDTPK & Phospho(ST) \\
\hline Q9Y6G9 & KPVTVSPTTPTSPTEGEAS & Phospho(ST) \\
\hline P35241 & DKYKTLR & Phospho(ST) \\
\hline Q9UPN6 & ETVQTTQSPTPVEK & Phospho(ST) \\
\hline P41743 & EGLRPGDTTSTFCGTPNYIAPEILR & Phospho(ST) \\
\hline Q8N3V7 & AASPAKPSSLDLVPNLPK & Phospho(ST) \\
\hline Q86YV5 & SASFAFEFPK & Phospho(ST) \\
\hline Q14155 & MSGFIYQGK & Phospho(ST) \\
\hline \multirow[t]{2}{*}{ Q9GZY8 } & SMSENAVR & Oxidation(M); \\
\hline & & Phospho(ST) \\
\hline P11274 & AAQAPDGASEPR & Phospho(ST) \\
\hline \multirow[t]{2}{*}{ P11274 } & ASASRPQPAPADGADPPPAEEPEARPDGEG & Phospho(ST) \\
\hline & SPGK & \\
\hline P18754 & RSPPADAIPK & Phospho(ST) \\
\hline P18754 & RRSPPADAIPK & Phospho(ST) \\
\hline Q99460 & TVGTPIASVPGSTNTGTVPGSEK & Phospho(ST) \\
\hline Q9NRX5 & SDGSLEDGDDVHR & Phospho(ST) \\
\hline Q9BX95 & NSLTGEEGQLAR & Phospho(ST) \\
\hline Q9BX95 & RNSLTGEEGQLAR & Phospho(ST) \\
\hline Q15365 & VMTIPYQPMPASSPVICAGGQDR & Phospho(ST) \\
\hline O75179 & NSPLDCGSASPNK & Phospho(ST) \\
\hline Q6P0Q8 & SWVIGSPEILR & Phospho(ST) \\
\hline Q9NSK0 & VSSPTVNTTLR & Phospho(ST) \\
\hline Q9H410 & IHLGSSPK & Phospho(ST) \\
\hline Q96ER3 & SSSDDEEQLTELDEEMENEICR & Phospho(ST) \\
\hline Q12929 & KGPGEGVLTLR & Phospho(ST) \\
\hline Q5T4S7 & HVTLPSSPR & Phospho(ST) \\
\hline Q5T4S7 & AAPPPPPPPPPLESSPR & Phospho(ST) \\
\hline Q9NZ53 & RDPEDSDVFEEDTHL & Phospho(ST) \\
\hline P50613 & AYTHQVVTR & Phospho(ST) \\
\hline P51946 & HEEEEWTDDDLVESL & Phospho(ST) \\
\hline Q96MH2 & TQSPGGCSAEAVLAR & Phospho(ST) \\
\hline A4D1S0 & VPVPESPAFSR & Phospho(ST) \\
\hline Q9P265 & YRSDIHTEAVQAALAK & Phospho(ST) \\
\hline Q15648 & LPSTSDDCPAIGTPLR & Phospho(ST) \\
\hline
\end{tabular}




\begin{tabular}{|c|c|c|}
\hline Protein & Peptide sequence & Variable modification \\
\hline Q15648 & LSSSDSIGPDVTDILSDIAEEASK & 2 Phospho(ST) \\
\hline Q15648 & $\begin{array}{l}\text { SHSIKPESWSKSPISSDQSLSMTSNTILSADR } \\
\text { PSR }\end{array}$ & 5 Phospho(ST) \\
\hline Q92625 & SLSKSDSDLLTCSPTEDATMGSR & Phospho(ST) \\
\hline Q8NCN4 & VLSPLIIK & Phospho(ST) \\
\hline Q9H7L9 & GRESDEDTEDASETDLAK & 3 Phospho(ST) \\
\hline Q9H7L9 & RPASPSSPEHLPATPAESPAQR & 2 Phospho(ST) \\
\hline Q13144 & GGSPQMDDIK & Phospho(ST) \\
\hline Q6PJG6 & GSPNTASAEATLPR & Phospho(ST) \\
\hline Q01850 & SSSETILSSLAGSDIVK & Phospho(ST) \\
\hline Q9NQW6 & TPISPLK & Phospho(ST) \\
\hline Q9NQW6 & $\begin{array}{l}\text { WDNDDMTDDIPESSLFSPMPSEEKAASPPR } \\
\text { PLLSNASATPVGRR }\end{array}$ & $\begin{array}{l}2 \text { Oxidation(M); } \\
2 \text { Phospho(ST) }\end{array}$ \\
\hline O60678 & $\begin{array}{l}\text { GAVENEEDLPELSDSGDEAAWEDEDDADL } \\
\text { PHGK }\end{array}$ & 2 Phospho(ST) \\
\hline P49327 & ADEASELACPTPK & Phospho(ST) \\
\hline P53667 & SPGAGSLGSPASQR & Phospho(ST) \\
\hline O14737 & KVMDSDEDDDY & $\begin{array}{l}\text { Oxidation(M); } \\
\text { Phospho(ST) }\end{array}$ \\
\hline Q9H6X2 & EVPPPPAEESEEEDDDGLPK & Phospho(ST) \\
\hline Q13443 & HVSPVTPPR & Phospho(ST) \\
\hline Q9BRK4 & AAAGVGGSLR & Phospho(ST) \\
\hline Q53EP0 & LNSPPSSIYK & Phospho(ST) \\
\hline P18887 & TKPTQAAGPSSPQKPPTPEETK & Phospho(ST) \\
\hline P18887 & TKPTQAAGPSSPQKPPTPEETK & 2 Phospho(ST) \\
\hline Q9H8Y5 & EALGQNEESPK & Phospho(ST) \\
\hline Q9H8Y5 & TSCSGSGERESPERK & Phospho(ST) \\
\hline Q9BVS4 & EGSEFSFSDGEVAEK & 3 Phospho(ST) \\
\hline Q6U841 & SFADIGK & Phospho(ST) \\
\hline O15234 & STVTGERQSGDGQESTEPVENK & Phospho(ST) \\
\hline Q8TEM1 & DLAVPAALTPR & Phospho(ST) \\
\hline Q14498 & YRSPYSGPK & Phospho(ST) \\
\hline Q14498 & DKSPVREPIDNLTPEER & Phospho(ST) \\
\hline Q96PK6 & TRLSPPR & Phospho(ST) \\
\hline Q96PK6 & QPTPPFFGR & Phospho(ST) \\
\hline O60292 & TLSDESLCSGR & Phospho(ST) \\
\hline O60333 & MSDTGSPGMQR & Phospho(ST) \\
\hline P45973 & TADSSSSEDEEEYVVEK & 4 Phospho(ST) \\
\hline Q02241 & YMLTHQELASDGEIETK & Phospho(ST) \\
\hline P78347 & SILSPGGSCGPIK & Phospho(ST) \\
\hline Q9ULW0 & SSDQPLTVPVSPK & Phospho(ST) \\
\hline Q8IY81 & ALDISLSSGEEDEGDEEDSTAGTTK & 2 Phospho(ST) \\
\hline Q7L4I2 & EQSEVSVSPR & Phospho(ST) \\
\hline
\end{tabular}




\begin{tabular}{|c|c|c|}
\hline Protein & Peptide sequence & Variable modification \\
\hline Q07157 & SREDLSAQPVQTK & Phospho(ST) \\
\hline Q07157 & VQIPVSRPDPEPVSDNEEDSYDEEIHDPR & 2 Phospho(ST) \\
\hline Q8N556 & SGTSSPQSPVFR & Phospho(ST) \\
\hline Q5TKA1 & FFMTPPR & Phospho(ST) \\
\hline Q07960 & SSSPELVTHLK & Phospho(ST) \\
\hline Q9C0D6 & ASSARAPK & Phospho(ST) \\
\hline Q9H0B6 & ASSLNFLNK & Phospho(ST) \\
\hline P0C1Z6 & LLPYPTLASPASD & Phospho(ST) \\
\hline $\mathrm{O} 00178$ & SAMDSPVPASMFAPEPSSPGAAR & Phospho(ST) \\
\hline Q14004 & SLSPLGGR & 2 Phospho(ST) \\
\hline Q14004 & IEHAPSPSSGGTLK & Phospho(ST) \\
\hline Q14004 & TNTPQGVLPSSQLK & Phospho(ST) \\
\hline P49761 & YRSPEPDPYLSYR & Phospho(ST) \\
\hline \multirow[t]{2}{*}{ Q9NRL3 } & LGGSVLGQIPFLQNCEDEDSDEDDELDSVQ & Phospho(ST) \\
\hline & HK & \\
\hline Q5JSZ5 & EAGSPAQEFK & Phospho(ST) \\
\hline Q7Z6E9 & LEVTEIVKPSPK & Phospho(ST) \\
\hline Q7Z6E9 & DDATPVRDEPMDAESITFK & Phospho(ST) \\
\hline Q9H0D6 & AEDSDSEPEPEDNVR & 2 Phospho(ST) \\
\hline Q9H0D6 & KAEDSDSEPEPEDNVR & 2 Phospho(ST) \\
\hline Q96T58 & NVDAAVSPR & Phospho(ST) \\
\hline Q96T58 & SPSGSGSRSR & Phospho(ST) \\
\hline Q96T58 & SQSPVHLR & 2 Phospho(ST) \\
\hline Q96T58 & DLEPGEVPSDSDEDGEHK & 2 Phospho(ST) \\
\hline Q01130 & RESKSR & Phospho(ST) \\
\hline Q01130 & SKSPPKSPEEEGAVSS & 3 Phospho(ST) \\
\hline Q01130 & SKSPPKSPEEEGAVSS & 4 Phospho(ST) \\
\hline Q9Y463 & IYQYIQSR & Phospho(Y) \\
\hline P13051 & HAPSPEPAVQGTGVAGVPEESGDAAAIPAK & Phospho(ST) \\
\hline Q86V48 & EKPDSDDDLDIASLVTAK & Phospho(ST) \\
\hline Q14839 & FGTEELFK & Phospho(ST) \\
\hline Q99081 & GNAAGSSQTGDALGK & Phospho(ST) \\
\hline O75822 & VLTPEEQLADK & Phospho(ST) \\
\hline Q8NFC6 & TQLSPSIK & Phospho(ST) \\
\hline Q8NFC6 & DKDVTLSPVK & Phospho(ST) \\
\hline Q9NPF5 & DTIIDVVGAPLTPNSR & Phospho(ST) \\
\hline Q8NHG8 & DRPVGGSPGGPR & Phospho(ST) \\
\hline A0MZ66 & KVTAEADSSSPTGILATSESK & Phospho(ST) \\
\hline Q9BUR4 & GDPPRLSPDPVAGSAVSQELR & Phospho(ST) \\
\hline Q9BW71 & RPPTPCSDPER & 2 Phospho(ST) \\
\hline Q9BW71 & TLDSDEERPRPAPPDWSHMR & Phospho(ST) \\
\hline P12268 & LVGIISSR & Phospho(ST) \\
\hline Q01804 & EESSEDENEVSNILR & 2 Phospho(ST) \\
\hline
\end{tabular}




\begin{tabular}{|c|c|c|}
\hline Protein & Peptide sequence & Variable modification \\
\hline Q8N3F8 & VEQMPQASPGLAPR & Phospho(ST) \\
\hline Q15773 & LAIQGPEDSPSR & Phospho(ST) \\
\hline Q63HN8 & EAAEPLSEPKEDQEAAELLSEPEEESER & Phospho(ST) \\
\hline Q9Y2X7 & HGSGADSDYENTQSGDPLLGLEGK & 2 Phospho(ST) \\
\hline P17535 & $\begin{array}{l}\text { LAALKDEPQTVPDVPSFGESPPLSPIDMDT } \\
\text { QER }\end{array}$ & Phospho(ST) \\
\hline Q8IY67 & EALGLGPPAAQLTPPPAPVGLR & Phospho(ST) \\
\hline Q8IY67 & VSFCAPGPPGRSMLAALIAAQATALNRGK & $\begin{array}{l}\text { Oxidation(M); } \\
\text { Phospho(ST) }\end{array}$ \\
\hline Q9P035 & WLDESDAEMELR & $\begin{array}{l}\text { Oxidation(M); } \\
\text { Phospho(ST) }\end{array}$ \\
\hline Q15287 & RFSPPR & Phospho(ST) \\
\hline Q15287 & RRSPSPKPTK & 2 Phospho(ST) \\
\hline Q8ND76 & SASADNLTLPR & Phospho(ST) \\
\hline Q9UKM9 & GRLSPVPVPR & Phospho(ST) \\
\hline Q9UKM9 & $\begin{array}{l}\text { DDGDEEGLLTHSEEELEHSQDTDADDGAL } \\
\text { Q }\end{array}$ & Phospho(ST) \\
\hline Q9UKM9 & $\begin{array}{l}\text { TRDDGDEEGLLTHSEEELEHSQDTDADDG } \\
\text { ALQ }\end{array}$ & 2 Phospho(ST) \\
\hline Q99549 & GAEAFGDSEEDGEDVFEVEK & Phospho(ST) \\
\hline Q7L2J0 & TLNAETPKSSPLPAK & 2 Phospho(ST) \\
\hline P63208 & GKTPEEIR & Phospho(ST) \\
\hline Q5BKZ1 & ISLSKSPTK & Phospho(ST) \\
\hline Q6R327 & STELLLGVK & Phospho(ST) \\
\hline Q6R327 & TLTEPSVDFNHSDDFTPISTVQK & Phospho(ST) \\
\hline P62136 & YGQFSGLNPGGRPITPPR & Phospho(ST) \\
\hline Q9NQS7 & VLAPILPDNFSTPTGSR & Phospho(ST) \\
\hline Q92878 & VFQTEAELQEVISDLQSK & Phospho(ST) \\
\hline Q8NDT2 & ASGDPGASGMSPR & $\begin{array}{l}\text { Oxidation(M); } \\
\text { Phospho(ST) }\end{array}$ \\
\hline Q9H5H4 & TQSPEFEAQSSK & Phospho(ST) \\
\hline Q9Y3Z3 & NFTKPQDGDVIAPLITPQKK & Phospho(ST) \\
\hline Q86TB9 & STSPIIGSPPVR & Phospho(ST) \\
\hline Q13017 & GGIDNPAITSDQELDDKK & Phospho(ST) \\
\hline Q13435 & GFEEEHKDSDDDSSDDEQEKKPEAPK & 3 Phospho(ST) \\
\hline P24394 & SSPPTTPLR & Phospho(ST) \\
\hline Q9C0D5 & GVSMSLPSSPLLPR & 2 Phospho(ST) \\
\hline Q68EM7 & SPSPPTQHTGQPPGQPSAPSQLSAPR & Phospho(ST) \\
\hline Q86YP4 & RPPSPDVIVLSDNEQPSSPR & 3 Phospho(ST) \\
\hline Q9ULJ3 & SLSMDSQVPVYSPSIDLK & Phospho(ST) \\
\hline Q8WXI9 & VSSPLPSPSAMTDAANSQAAAK & Phospho(ST) \\
\hline Q04726 & DAPTSPASVASSSSTPSSK & Phospho(ST) \\
\hline Q9UKJ3 & GPKPEPPGSGSPAPPR & Phospho(ST) \\
\hline
\end{tabular}




\begin{tabular}{|c|c|c|}
\hline Protein & Peptide sequence & Variable modification \\
\hline Q8WU79 & DLDLLASVPSPSSSGSR & Phospho(ST) \\
\hline Q07866 & VDSPTVTTTLK & Phospho(ST) \\
\hline P78563 & NLDNVSPK & Phospho(ST) \\
\hline Q2KHR3 & NLEHLSSFSSDEDDPGYSQDAYK & 2 Phospho(ST) \\
\hline Q7L7X3 & TASLVTR & Phospho(ST) \\
\hline O95573 & IRLSPEPWTPETGLVTDAFK & Phospho(ST) \\
\hline Q8WVB6 & VENSPQVDGSPPGLEGLLGGIGEK & Phospho(ST) \\
\hline Q5TDH0 & QPPGTQQSHSSPGEITSSPQGLDNPALLR & Phospho(ST) \\
\hline P51116 & GPPPAPRPTSR & Phospho(ST) \\
\hline P51116 & TDGSISGDRQPVTVADYISR & Phospho(ST) \\
\hline Q86U44 & $\begin{array}{l}\text { SDSPVPTAPTSGGPKPSTASAVPELATDPEL } \\
\text { EK }\end{array}$ & Phospho(ST) \\
\hline Q86U44 & $\begin{array}{l}\text { SDSPVPTAPTSGGPKPSTASAVPELATDPEL } \\
\text { EKK }\end{array}$ & Phospho(ST) \\
\hline Q14814 & VIPAKSPPPPTHSTQLGAPSR & Phospho(ST) \\
\hline Q00536 & RLSLPADIR & Phospho(ST) \\
\hline Q00536 & RVSLSEIGFGK & Phospho(ST) \\
\hline Q9NRG9 & FSPVLGR & Phospho(ST) \\
\hline P16989 & $\begin{array}{l}\text { SPVGSGAPQAAAPAPAAHVAGNPGGDAAP } \\
\text { AATGTAAAASLATAAGSEDAEKK }\end{array}$ & Phospho(ST) \\
\hline Q9BYW2 & LNDSPTLK & Phospho(ST) \\
\hline O43379 & $\begin{array}{l}\text { RPSSVGELASLGQELQAITTATTPSLDSEGQ } \\
\text { EPALR }\end{array}$ & Phospho(ST) \\
\hline P43487 & $\begin{array}{l}\text { DTHEDHDTSTENTDESNHDPQFEPIVSLPE } \\
\text { QEIK }\end{array}$ & Phospho(ST) \\
\hline Q15527 & DLGSTEDGDGTDDFLTDKEDEK & 2 Phospho(ST) \\
\hline Q15527 & KDLGSTEDGDGTDDFLTDKEDEK & Phospho(ST) \\
\hline O95831 & SLSAIDR & Phospho(ST) \\
\hline Q9P2E9 & SHVEDGDIAGAPASSPEAPPAEQDPVQLK & Phospho(ST) \\
\hline Q5M775 & SPLSGIPVR & Phospho(ST) \\
\hline Q765P7 & ALSSAGPIPIRPPIVPVK & Phospho(ST) \\
\hline Q16649 & SSDGEDEQQVPK & Phospho(ST) \\
\hline P00519 & GQGESDPLDHEPAVSPLLPR & Phospho(ST) \\
\hline Q16204 & SNSPDKFK & Phospho(ST) \\
\hline Q16204 & SGGIVISPFR & Phospho(ST) \\
\hline Q96HH9 & ADRPSSLPLDFNDEFSDLDGVVQQR & Phospho(ST) \\
\hline Q9BQE9 & VDSSTNSSPSPQQSESLSPAHTSDFR & Phospho(ST) \\
\hline Q9H8G2 & SVNEILGLAESSPNEPK & Phospho(ST) \\
\hline Q03252 & LKLSPSPSSR & Phospho(ST) \\
\hline Q03252 & AGGPATPLSPTR & Phospho(ST) \\
\hline P55884 & $\begin{array}{l}\text { TEPAAEAEAASGPSESPSPPAAEELPGSHAE } \\
\text { PPVPAQGEAPGEQAR }\end{array}$ & Phospho(ST) \\
\hline Q03701 & SQLDDHPESDDEENFIDANDDEDMEK & Phospho(ST) \\
\hline
\end{tabular}




\begin{tabular}{|c|c|c|}
\hline Protein & Peptide sequence & Variable modification \\
\hline P51636 & ADVQLFMDDDSYSHHSGLEYADPEK & 2 Phospho(ST) \\
\hline P51636 & ADVQLFMDDDSYSHHSGLEYADPEK & 3 Phospho(ST) \\
\hline Q53GS9 & SKRESR & Phospho(ST) \\
\hline Q53GS9 & EREVDEDSEPER & Phospho(ST) \\
\hline P13807 & HSSPHQSEDEEDPR & 2 Phospho(ST) \\
\hline Q86UU1 & ALSPLPTR & Phospho(ST) \\
\hline Q86UU1 & TFSDGLATR & Phospho(ST) \\
\hline Q86UU1 & TLQPPESPR & Phospho(ST) \\
\hline Q9UNZ2 & KKSPNELVDDLFK & Phospho(ST) \\
\hline O95671 & VVLASASPR & Phospho(ST) \\
\hline O95671 & HDSIPAADTFEDLSDVEGGGSEPTQR & Phospho(ST) \\
\hline P11171 & LFSSFLK & Phospho(ST) \\
\hline P15311 & DKYKTLR & Phospho(ST) \\
\hline Q9BXS5 & LETGAPRPPATVTNAVSWR & Phospho(ST) \\
\hline O14686 & ALSPVIPLIPR & Phospho(ST) \\
\hline Q3KR37 & SSIETKPDASPQLPK & Phospho(ST) \\
\hline Q99816 & DGTISEDTIR & Phospho(ST) \\
\hline Q9Y6D9 & AILGSYDSELTPAEYSPQLTR & Phospho(ST) \\
\hline O96013 & DKRPLSGPDVGTPQPAGLASGAK & Phospho(ST) \\
\hline Q99547 & HKAEEVEDETVELDVSDEEMAR & Phospho(ST) \\
\hline Q9BQA1 & KETPPPLVPPAAR & Phospho(ST) \\
\hline O94804 & LSEEAECPNPSTPSK & Phospho(ST) \\
\hline O43159 & TEVSPVPR & Phospho(ST) \\
\hline O15541 & AAYGDLSSEEEEENEPESLGVVYK & 2 Phospho(ST) \\
\hline P04049 & SASEPSLHR & Phospho(ST) \\
\hline Q00613 & VKEEPPSPPQSPR & 2 Phospho(ST) \\
\hline Q9P2B4 & VSSPLSPLSPGIKSPTIPR & Phospho(ST) \\
\hline Q9BTU6 & VAAAAGSGPSPPGSPGHDR & 2 Phospho(ST) \\
\hline Q86VQ1 & GSQHSPTRPPVAAAAASLGSLPGPGAAR & Phospho(ST) \\
\hline P43243 & RDSFDDR & Phospho(ST) \\
\hline Q9Y5N6 & IGQQVDREPGDVATPPR & Phospho(ST) \\
\hline Q15366 & QICVVMLETLSQSPPK & Phospho(ST) \\
\hline Q71RC2 & DGLNQTTIPVSPPSTTKPSR & Phospho(ST) \\
\hline \multirow[t]{2}{*}{ O43615 } & YKTIESETVR & $2 \quad$ Phospho(ST); \\
\hline & & Phospho(Y) \\
\hline Q86W50 & ELSLKASPLR & Phospho(ST) \\
\hline Q9BTC0 & GSAPFSEK & Phospho(ST) \\
\hline Q9BTC0 & GGDDHDDTSDSDSDGLTLK & 3 Phospho(ST) \\
\hline P15408 & QEPLEEDSPSSSSAGLDK & Phospho(ST) \\
\hline Q8WX92 & KPSPAQAAETPALELPLPSVPAPAPL & Phospho(ST) \\
\hline P51610 & RPMSSPEMK & Phospho(ST) \\
\hline Q96BT3 & ALLETASPR & Phospho(ST) \\
\hline Q03135 & AMADELSEK & Phospho(ST) \\
\hline
\end{tabular}




\begin{tabular}{|c|c|c|}
\hline Protein & Peptide sequence & Variable modification \\
\hline Q13200 & APVQPQQSPAAAPGGTDEKPSGK & Phospho(ST) \\
\hline Q13200 & DKAPVQPQQSPAAAPGGTDEKPSGK & Phospho(ST) \\
\hline P43307 & $\begin{array}{l}\text { VEMGTSSQNDVDMSWIPQETLNQINKASP } \\
\mathrm{R}\end{array}$ & Phospho(ST) \\
\hline P43307 & $\begin{array}{l}\text { VEMGTSSQNDVDMSWIPQETLNQINKASP } \\
\mathrm{R}\end{array}$ & $\begin{array}{l}\text { Oxidation(M); } \\
\text { Phospho(ST) }\end{array}$ \\
\hline Q9UHI6 & YQESPGIQMK & Phospho(ST) \\
\hline Q6WCQ1 & SKSNPDFLKK & Phospho(ST) \\
\hline Q08945 & SKEFVSSDESSSGENK & 4 Phospho(ST) \\
\hline Q08945 & EGMNPSYDEYADSDEDQHDAYLER & $\begin{array}{l}\text { Oxidation(M); } \\
\text { Phospho(ST) }\end{array}$ \\
\hline Q9BZE4 & TAAGEYDSVSESEDEEMLEIR & $\begin{array}{l}2 \text { Phospho(ST); } \\
\text { Phospho(Y) }\end{array}$ \\
\hline P23528 & LGGSAVISLEGKPL & Phospho(ST) \\
\hline Q96TA1 & AAPEASSPPASPLQHLLPGK & 2 Phospho(ST) \\
\hline Q8N201 & RDSTEAPKPK & Phospho(ST) \\
\hline Q8N201 & LSSTPPLSALGR & Phospho(ST) \\
\hline Q6ZNE9 & EVIGMEAEVTGVLLVAEGQR & Phospho(ST) \\
\hline Q8WUA2 & INHTVILDDPFDDPPDLLIPDRSPEPTR & Phospho(ST) \\
\hline Q99504 & LSSGDPSTSPSLSQTTPSKDTDDQSR & Phospho(ST) \\
\hline Q9BUJ2 & APQQQPPPQQPPPPQPPPQQPPPPPSYSPAR & Phospho(ST) \\
\hline Q9NWB6 & SRESKR & Phospho(ST) \\
\hline Q9NWB6 & ASSPPDRIDIFGR & Phospho(ST) \\
\hline Q96GN5 & EVADIFNAPSDDEEFVGFR & Phospho(ST) \\
\hline Q8NDX5 & MDRTPPPPTLSPAAITVGR & Phospho(ST) \\
\hline O95835 & QITTSPITVR & Phospho(ST) \\
\hline Q9UPP1 & DAEYIYPSLESDDDDPALK & Phospho(ST) \\
\hline Q8IXS8 & VEVTPTVPR & Phospho(ST) \\
\hline Q9NZT2 & SQGDEAGGHGEDRPEPLSPK & Phospho(ST) \\
\hline P46087 & GPQPPTVSPIR & Phospho(ST) \\
\hline P46087 & EAAAGIQWSEEETEDEEEEKEVTPESGPPK & 2 Phospho(ST) \\
\hline Q14680 & VFGSLER & Phospho(ST) \\
\hline Q9BUA3 & AESPSPAPPPGLR & Phospho(ST) \\
\hline Q9BUA3 & AEQPSPPNSDSGQDAHPDPDANPDAAR & 2 Phospho(ST) \\
\hline P83916 & KADSDSEDKGEESKPK & Phospho(ST) \\
\hline Q8IXT5 & SPTEDFR & Phospho(ST) \\
\hline O00629 & NVPHEDICEDSDIDGDYR & Phospho(ST) \\
\hline Q9BRQ0 & GGGTPDANSLAPPGK & Phospho(ST) \\
\hline Q02750 & LCDFGVSGQLIDSMANSFVGTR & Phospho(ST) \\
\hline O43290 & RVSEVEEEKEPVPQPLPSDDTR & Phospho(ST) \\
\hline P46020 & $\begin{array}{l}\text { LSISAESQSPGTSMTPSSGSFPSAYDQQSSK } \\
\text { DSR }\end{array}$ & 3 Phospho(ST) \\
\hline Q6ZUS6 & SSSSALTGVLSAK & Phospho(ST) \\
\hline
\end{tabular}




\begin{tabular}{|c|c|c|}
\hline Protein & Peptide sequence & Variable modification \\
\hline A6NFI3 & GGDAKSPVLQEK & Phospho(ST) \\
\hline Q4ADV7 & SISLSQSAENVPASK & Phospho(ST) \\
\hline Q13098 & EGSQGELTPANSQSR & Phospho(ST) \\
\hline Q06210 & VDSTTCLFPVEEK & Phospho(ST) \\
\hline P60468 & PGPTPSGTNVGSSGRSPSK & Phospho(ST) \\
\hline Q9BQ67 & MHNLHGTKPPPSEGSDEEEEEEDEEDEEER & 2 Phospho(ST) \\
\hline P02794 & MGAPESGLAEYLFDKHTLGDSDNES & Phospho(ST) \\
\hline Q96ST3 & GDLSDVEEEEEEEMDVDEATGAVK & Phospho(ST) \\
\hline O60524 & DELNEELIQEESSEDEGEYEEVRK & 2 Phospho(ST) \\
\hline Q8WW12 & NIGRDTPTSAGPNSFNK & Phospho(ST) \\
\hline Q8NFH5 & SVMESSDR & Phospho(ST) \\
\hline Q8NFH5 & SPLLAGGSPPQPVVPAHK & Phospho(ST) \\
\hline \multirow[t]{2}{*}{ Q96DN2 } & QHPSKMLLLLPEAGRPALSPGHSPPSGAPG & Oxidation(M); \\
\hline & PPAGVR & Phospho(ST) \\
\hline \multirow[t]{2}{*}{ Q86U70 } & KMSGGSTMSSGGGNTNNSNSK & 2 Oxidation $(\mathrm{M})$ \\
\hline & & Phospho(ST) \\
\hline P98082 & SSPNPFVGSPPK & Phospho(ST) \\
\hline Q68E01 & EKFPEFCSSPSPPVEVK & Phospho(ST) \\
\hline Q6UUV7 & LTQYHGGSLPNVSQLR & Phospho(ST) \\
\hline P42696 & SVQEGENPDDGVR & Phospho(ST) \\
\hline P49815 & GQPEGPLPSSSPR & Phospho(ST) \\
\hline P54725 & EDKSPSEESAPTTSPESVSGSVPSSGSSGR & Phospho(ST) \\
\hline Q9NYV4 & HLLTDLPLPPELPGGDLSPPDSPEPK & 2 Phospho(ST) \\
\hline Q13042 & NIISPPWDFR & Phospho(ST) \\
\hline P31321 & SNSQSDSHDEEVSPTPPNPVVK & Phospho(ST) \\
\hline Q03164 & TIAHSPTSFTESSSK & Phospho(ST) \\
\hline Q92974 & LQDSSDPDTGSEEEGSSRLSPPHSPR & 2 Phospho(ST) \\
\hline Q92597 & TASGSSVTSLDGTR & 2 Phospho(ST) \\
\hline Q13177 & YLSFTPPEKDGFPSGTPALNAK & Phospho(ST) \\
\hline \multirow[t]{2}{*}{ Q13177 } & GTEAPAVVTEEEDDDEETAPPVIAPRPDHT & Phospho(ST) \\
\hline & $\mathrm{K}$ & \\
\hline Q8IWX8 & SRSRSPTPPSSAGLGSNSAPPIPDSR & 4 Phospho(ST) \\
\hline Q9H4L5 & LHSSNPNLSTLDFGEEK & Phospho(ST) \\
\hline O95456 & AGTEDEEEEEEGRR & Phospho(ST) \\
\hline Q8TEU7 & LPEGPVDSEDDEEEDEEIDRTDPLQGR & Phospho(ST) \\
\hline O60245 & LSDSPSMGR & Phospho(ST) \\
\hline O60245 & SSSPLPTVQLHPQSPTAGK & Phospho(ST) \\
\hline Q13242 & GSPHYFSPFRPY & 2 Phospho(ST) \\
\hline P55201 & GAGWLSEDEDSPLDALDLVWAK & Phospho(ST) \\
\hline Q9Y4B5 & VLHSPPAVR & Phospho(ST) \\
\hline P54259 & SEEISESESEETNAPK & 2 Phospho(ST) \\
\hline Q8NCF5 & KTEFLDLDNSPLSPPSPR & Phospho(ST) \\
\hline Q9NYB9 & HTPPTIGGSLPYR & Phospho(ST) \\
\hline
\end{tabular}




\begin{tabular}{|c|c|c|c|}
\hline Protein & Peptide sequence & Variable modification & \\
\hline Q9NYB9 & TTPPTQKPPSPPMSGK & Phospho(ST) & \\
\hline \multirow[t]{3}{*}{ P07858 } & HYGYNSYSVSNSEKDIMAEIYK & Oxidation(M); & \\
\hline & & Phospho(ST); & 4 \\
\hline & & Phospho(Y) & \\
\hline Q8TAP8 & APVPEPGLDLSLSPRPDSPQPR & Phospho(ST) & \\
\hline Q9UBS5 & TGSSTNNNEEEKSR & 2 Phospho(ST) & \\
\hline Q86X95 & NLTANDPSQEYVASEGEEDPEVEFLK & Phospho(ST) & \\
\hline O43474 & TTPTLGLEEVLSSR & Phospho(ST) & \\
\hline Q12889 & LAVTTAWTTDSKILPPGGEAGVTEIHGK & 3 Phospho(ST) & \\
\hline Q86WR0 & VENMSSNQDGNDSDEFM & Phospho(ST) & \\
\hline O14497 & TLLDPGRFSK & Phospho(ST) & \\
\hline O14497 & GPSPSPVGSPASVAQSR & Phospho(ST) & \\
\hline P83369 & SAGAGSPARPPSPR & 2 Phospho(ST) & \\
\hline Q07666 & SGSMDPSGAHPSVR & Phospho(ST) & \\
\hline Q15022 & ASMSEFLESEDGEVEQQR & Phospho(ST) & \\
\hline O15164 & SEWLDPSQKSPLHVGETR & Phospho(ST) & \\
\hline P82094 & SATPVNCEQPDILVSSTPINEGQTVLDK & Phospho(ST) & \\
\hline O15318 & GDGEKSDEENEEKEGSK & Phospho(ST) & \\
\hline Q96FF9 & APSPTKPLR & Phospho(ST) & \\
\hline P05114 & TEESPASDEAGEKEAKSD & Phospho(ST) & \\
\hline P06748 & SIRDTPAK & Phospho(ST) & \\
\hline P06748 & GPSSVEDIK & Phospho(ST) & \\
\hline P06748 & TVSLGAGAKDELHIVEAEAMNYEGSPIK & Phospho(ST) & \\
\hline Q09666 & VDVKSPK & Phospho(ST) & \\
\hline Q09666 & FGFGAKSPK & Phospho(ST) & \\
\hline Q09666 & DIDISSPEFK & Phospho(ST) & \\
\hline Q09666 & GGVTGSPEASISGSK & Phospho(ST) & \\
\hline Q09666 & GKGGVTGSPEASISGSK & Phospho(ST) & \\
\hline Q09666 & LPSGSGAASPTGSAVDIR & 2 Phospho(ST) & \\
\hline Q09666 & SKGHYEVTGSDDETGKLQGSGVSLASK & Phospho(ST) & \\
\hline P02545 & LRLSPSPTSQR & 2 Phospho(ST) & \\
\hline P02545 & ASASGSGAQVGGPISSGSSASSVTVTR & 2 Phospho(ST) & \\
\hline P20700 & LSPSPSSR & Phospho(ST) & \\
\hline P20700 & AGGPTTPLSPTR & Phospho(ST) & \\
\hline Q03252 & LSPSPSSR & Phospho(ST) & \\
\hline P07900 & EVSDDEAEEK & Phospho(ST) & \\
\hline P07900 & ESEDKPEIEDVGSDEEEEK & Phospho(ST) & \\
\hline Q9H1E3 & KTSTSPPPEK & Phospho(ST) & \\
\hline Q9H1E3 & NSQEDSEDSEDKDVK & Phospho(ST) & \\
\hline Q9H1E3 & NSQEDSEDSEDKDVK & 2 Phospho(ST) & \\
\hline Q9H1E3 & SGKNSQEDSEDSEDKDVK & 3 Phospho(ST) & \\
\hline Q9H1E3 & EKTPSPKEEDEEPESPPEK & Phospho(ST) & \\
\hline P10412 & SETAPAAPAAPAPAEKTPVKK & Phospho(ST) & \\
\hline
\end{tabular}




\begin{tabular}{|c|c|c|}
\hline Protein & Peptide sequence & Variable modification \\
\hline P16401 & KVAKSPK & Phospho(ST) \\
\hline P16401 & ATKSPAKPK & Phospho(ST) \\
\hline P16402 & VKTPQPK & Phospho(ST) \\
\hline P16402 & KVAGAATPK & Phospho(ST) \\
\hline P16403 & KVAKSPK & Phospho(ST) \\
\hline P16403 & KASGPPVSELITK & Phospho(ST) \\
\hline Q9UQ35 & CLTPQR & Phospho(ST) \\
\hline Q9UQ35 & SSPELTR & Phospho(ST) \\
\hline Q9UQ35 & SRASPVSR & Phospho(ST) \\
\hline Q9UQ35 & SPSPASGRR & Phospho(ST) \\
\hline Q9UQ35 & VKPETPPR & Phospho(ST) \\
\hline Q9UQ35 & SGMSPEQSR & Phospho(ST) \\
\hline Q9UQ35 & SRSPLAIR & 2 Phospho(ST) \\
\hline \multirow[t]{2}{*}{ Q9UQ35 } & SGMSPEQSR & Oxidation(M); \\
\hline & & Phospho(ST) \\
\hline Q9UQ35 & RPSPQPSPR & Phospho(ST) \\
\hline Q9UQ35 & RVPSPTPAPK & Phospho(ST) \\
\hline Q9UQ35 & HSCSGSSPPR & Phospho(ST) \\
\hline \multirow{2}{*}{ Q9UQ35 } & GEFSASPMLK & Oxidation(M); \\
\hline & & Phospho(ST) \\
\hline Q9UQ35 & HSLSGSSPGMK & Phospho(ST) \\
\hline Q9UQ35 & SLSYSPVER & 3 Phospho(ST) \\
\hline Q9UQ35 & SPGMLEPLGSSR & Phospho(ST) \\
\hline Q9UQ35 & YSHSGSSSPDTK & Phospho(ST) \\
\hline Q9UQ35 & RRPSPQPSPR & 2 Phospho(ST) \\
\hline Q9UQ35 & SVSPCSNVESR & 2 Phospho(ST) \\
\hline Q9UQ35 & SCFESSPDPELK & Phospho(ST) \\
\hline \multirow[t]{2}{*}{ Q9UQ35 } & AGMSSNQSISSPVLDAVPRTPSR & Oxidation(M); \\
\hline & & 2 Phospho(ST) \\
\hline Q9UQ35 & HGGSPQPLATTPLSQEPVNPPSEASPTR & 2 Phospho(ST) \\
\hline Q8IYB3 & VSHSPPPK & Phospho(ST) \\
\hline Q8IYB3 & TASPPPPPK & Phospho(ST) \\
\hline Q8IYB3 & AASPSPQSVR & Phospho(ST) \\
\hline Q8IYB3 & AASPSPQSVR & 2 Phospho(ST) \\
\hline Q8IYB3 & RVSHSPPPK & 2 Phospho(ST) \\
\hline Q8IYB3 & RSPSPAPPPR & 2 Phospho(ST) \\
\hline Q8IYB3 & RLSPSASPPR & 2 Phospho(ST) \\
\hline Q8IYB3 & RVSRTPEPK & 2 Phospho(ST) \\
\hline Q8IYB3 & RASPSPPPKR & 2 Phospho(ST) \\
\hline Q8IYB3 & KAASPSPQSVR & 2 Phospho(ST) \\
\hline Q8IYB3 & KVELSESEEDK & Phospho(ST) \\
\hline Q8IYB3 & TRHSPTPQQSNR & 2 Phospho(ST) \\
\hline Q8IYB3 & KPPAPPSPVQSQSPSTNWSPAVPVK & 3 Phospho(ST) \\
\hline
\end{tabular}




\begin{tabular}{|c|c|c|}
\hline Protein & Peptide sequence & Variable modification \\
\hline P51858 & KGNAEGSSDEEGKLVIDEPAK & 2 Phospho(ST) \\
\hline Q15149 & AQLEPVASPAK & Phospho(ST) \\
\hline Q15149 & TPHVQAVQGPLGSPPKR & Phospho(ST) \\
\hline Q15149 & SSSVGSSSSYPISPAVSR & Phospho(ST); Phospho(Y) \\
\hline \multirow[t]{2}{*}{ Q15149 } & GFFDPNTEENLTYLQLMERCITDPQTGLCL & Oxidation(M); \\
\hline & LPLK & 2 Phospho(ST) \\
\hline P42166 & LREQGTESR & Phospho(ST) \\
\hline P42166 & SSTPLPTISSSAENTR & 2 Phospho(ST) \\
\hline P42167 & LREQGTESR & Phospho(ST) \\
\hline P42167 & SSTPLPTISSSAENTR & 2 Phospho(ST) \\
\hline P21333 & CSGPGLSPGMVR & Phospho(ST) \\
\hline Q13428 & KLSGDQPAAR & Phospho(ST) \\
\hline Q13428 & AASAPAKESPR & Phospho(ST) \\
\hline Q13428 & LDSSPSVSSTLAAK & Phospho(ST) \\
\hline Q13428 & KLGAGEGGEASVSPEK & Phospho(ST) \\
\hline P62258 & AAFDDAIAELDTLSEESYK & Phospho(ST) \\
\hline P08670 & SYVTTSTR & Phospho(ST) \\
\hline P08670 & ISLPLPNFSSLNLR & Phospho(ST) \\
\hline P08670 & DGQVINETSQHHDDLE & Phospho(ST) \\
\hline P05787 & SYTSGPGSR & Phospho(ST) \\
\hline P05787 & YEELQSLAGK & Phospho(ST) \\
\hline Q99613 & QPLLLSEDEEDTK & Phospho(ST) \\
\hline B5ME19 & QPLLLSEDEEDTK & Phospho(ST) \\
\hline P18615 & SISADDDLQESSR & Phospho(ST) \\
\hline O43719 & LFEDDDSNEK & Phospho(ST) \\
\hline O43719 & GSEEDSPKKESK & Phospho(ST) \\
\hline O43719 & LFEESDDKEDEDADGKEVEDADEK & Phospho(ST) \\
\hline O43719 & LFEDDDSNEKLFDEEEDSSEKLFDDSDER & 3 Phospho(ST) \\
\hline Q7Z417 & TSPQVLGSILK & Phospho(ST) \\
\hline P49736 & TDALTSSPGR & Phospho(ST) \\
\hline P49736 & TDALTSSPGR & 2 Phospho(ST) \\
\hline P17096 & EPSEVPTPK & Phospho(ST) \\
\hline P17096 & KQPPVSPGTALVGSQK & Phospho(ST) \\
\hline \multirow[t]{2}{*}{ P24534 } & YGPADVEDTTGSGATDSKDDDDIDLFGSD & 2 Phospho(ST) \\
\hline & DEEESEEAKR & \\
\hline Q8WWI1 & GISSLPR & Phospho(ST) \\
\hline Q8WWI1 & WEAIREASR & Phospho(ST) \\
\hline Q8WWI1 & RGESLDNLDSPR & Phospho(ST) \\
\hline Q8WWI1 & EGFESDTDSEFTFK & Phospho(ST) \\
\hline Q8WWI1 & EVAATEEDVTRLPSPTSPFSSLSQDQAATSK & Phospho(ST) \\
\hline P11388 & KPSTSDDSDSNFEK & 4 Phospho(ST) \\
\hline P11388 & EAQQKVPDEEENEESDNEKETEK & Phospho(ST) \\
\hline Q02952 & SPPSPVER & Phospho(ST) \\
\hline
\end{tabular}




\begin{tabular}{|c|c|c|}
\hline Protein & Peptide sequence & Variable modification \\
\hline Q02952 & EVSSLEGSPPPCLGQEEAVCTK & Phospho(ST) \\
\hline Q02952 & $\begin{array}{l}\text { VELPSEEQVSGSQGPSEEKPAPLATEVFDE } \\
\mathrm{K}\end{array}$ & Phospho(ST) \\
\hline Q02952 & $\begin{array}{l}\text { LSAEYEKVELPSEEQVSGSQGPSEEKPAPL } \\
\text { ATEVFDEK }\end{array}$ & Phospho(ST) \\
\hline P67809 & NYQQNYQNSESGEKNEGSESAPEGQAQQR & 2 Phospho(ST) \\
\hline P19338 & VAVATPAK & Phospho(ST) \\
\hline P19338 & KVVVSPTK & Phospho(ST) \\
\hline P19338 & ALVATPGKK & Phospho(ST) \\
\hline P19338 & EVEEDSEDEEMSEDEEDDSSGEEVVIPQK & 4 Phospho(ST) \\
\hline O14974 & SGSYSYLEER & Phospho(ST) \\
\hline O14974 & FPTTATKISPK & Phospho(ST) \\
\hline Q9BZL4 & RSTQGVTLTDLKEAEK & Phospho(ST) \\
\hline P29966 & AEDGATPSPSNETPKK & Phospho(ST) \\
\hline P46821 & SSSPVKK & Phospho(ST) \\
\hline P46821 & SDISPLTPR & Phospho(ST) \\
\hline P46821 & ESSPLYSPTFSDSTSAVK & 2 Phospho(ST) \\
\hline P46821 & GEAEQSEEEADEEDKAEDAR & Phospho(ST) \\
\hline Q8NE71 & LSVPTSDEEDEVPAPKPR & 2 Phospho(ST) \\
\hline Q04695 & TSCRLSGGLGAGSCR & Phospho(ST) \\
\hline Q9NR30 & SDAGLESDTAMK & Phospho(ST) \\
\hline P27824 & QKSDAEEDGGTVSQEEEDR & 2 Phospho(ST) \\
\hline Q8WVC0 & KLTSDEEGEPSGK & 2 Phospho(ST) \\
\hline Q8WVC0 & YVISDEEEEDDD & Phospho(ST) \\
\hline Q8WVC0 & KNAIASDSEADSDTEVPK & 3 Phospho(ST) \\
\hline P27816 & KETERASPIK & Phospho(ST) \\
\hline P27816 & ASPSKPASAPASR & Phospho(ST) \\
\hline P27816 & DMSPLSETEMALGKDVTPPPETEVVLIK & Phospho(ST) \\
\hline O95218 & YNLDASEEEDSNK & Phospho(ST) \\
\hline O95218 & ENVEYIEREESDGEYDEFGR & Phospho(ST) \\
\hline P18583 & RDSSLR & Phospho(ST) \\
\hline P18583 & RSRTPSR & Phospho(ST) \\
\hline Q92688 & KRETDDEGEDD & Phospho(ST) \\
\hline Q8NHW5 & EESEESDEDMGFGLFD & Phospho(ST) \\
\hline O60841 & WDGSEEDEDNSK & Phospho(ST) \\
\hline O60841 & VEMYSGSDDDDDFNKLPK & 2 Phospho(ST) \\
\hline Q5VT52 & DVEDMELSDVEDDGSK & Phospho(ST) \\
\hline Q9H3N1 & SLGPSLATDKS & Phospho(ST) \\
\hline Q86WB0 & SMGTGDTPGLEVPSSPLR & Phospho(ST) \\
\hline \multirow[t]{2}{*}{ Q86WB0 } & SMGTGDTPGLEVPSSPLR & Oxidation(M); \\
\hline & & Phospho(ST) \\
\hline Q86WB0 & SQDATFSPGSEQAEKSPGPIVSR & Phospho(ST) \\
\hline Q8NC51 & FDQLFDDESDPFEVLK & Phospho(ST) \\
\hline
\end{tabular}




\begin{tabular}{|c|c|c|}
\hline Protein & Peptide sequence & Variable modification \\
\hline Q8NC51 & TDKSSASAPDVDDPEAFPALA & Phospho(ST) \\
\hline Q14247 & AKTQTPPVSPAPQPTEER & 2 Phospho(ST) \\
\hline O14950 & ATSNVFAMFDQSQIQEFK & 2 Phospho(ST) \\
\hline Q96ST2 & TIASDSEEEAGK & 2 Phospho(ST) \\
\hline Q96ST2 & GPASDSETEDASR & 2 Phospho(ST) \\
\hline Q96ST2 & HQASDSENEELPKPR & 2 Phospho(ST) \\
\hline P46013 & AFKESPK & Phospho(ST) \\
\hline P46013 & AQSLVISPPAPSPR & Phospho(ST) \\
\hline P46013 & IACKSPPPESVDTPTSTK & Phospho(ST) \\
\hline P55081 & MEREDSSEEEEEEIDDEEIER & 2 Phospho(ST) \\
\hline O95817 & SSTPLHSPSPIR & Phospho(ST) \\
\hline Q96T88 & IERPGEGSPMVDNPMR & Phospho(ST) \\
\hline Q99590 & KRPQSPSPR & 2 Phospho(ST) \\
\hline P54105 & EPVADEEEEDSDDDVEPITEFR & Phospho(ST) \\
\hline Q9Y2W1 & SPALKSPLQSVVVR & Phospho(ST) \\
\hline Q9Y2W1 & ERSPALKSPLQSVVVR & 2 Phospho(ST) \\
\hline Q9NTI5 & METVSNASSSSNPSSPGR & 2 Phospho(ST) \\
\hline O75962 & GAANASGSSPDAPAK & Phospho(ST) \\
\hline Q14103 & NEEDEGHSNSSPR & Phospho(ST) \\
\hline P25788 & ESLKEEDESDDDNM & Phospho(ST) \\
\hline Q01082 & RPPSPEPSTK & Phospho(ST) \\
\hline P47712 & NVSHNPLLLLTPQK & Phospho(ST) \\
\hline Q9HAU0 & GVISYQTLPR & Phospho(ST) \\
\hline $\mathrm{O} 15173$ & GLGAGAGAGEESPATSLPR & Phospho(ST) \\
\hline O75122 & VLNTGSDVEEAVADALK & Phospho(ST) \\
\hline Q14676 & $\begin{array}{l}\text { SSVKTPEPVVPTAPELQPSTSTDQPVTSEPT } \\
\text { YQATR }\end{array}$ & 2 Phospho(ST) \\
\hline Q9UK76 & $\begin{array}{l}\text { GEGDIHENVDTDLPGSLGQSEEKPVPAAPV } \\
\text { PSPVAPAPVPSR }\end{array}$ & Phospho(ST) \\
\hline Q96JM3 & TAPPASPEAR & Phospho(ST) \\
\hline Q96JM3 & KPSGSPDLWK & Phospho(ST) \\
\hline Q96JM3 & KTAPTLSPEHWK & Phospho(ST) \\
\hline Q96JM3 & KTSPASLDFPESQK & Phospho(ST) \\
\hline Q96JM3 & CDILVQEELLASPK & Phospho(ST) \\
\hline Q96JM3 & LAPVPSPEPQKPAPVSPESVK & 2 Phospho(ST) \\
\hline Q96JM3 & KPSPSESPEPWKPFPAVSPEPR & Phospho(ST) \\
\hline Q8NFC6 & SLKHSSK & Phospho(ST) \\
\hline Q8NFC6 & GLEGNANSPAHLR & Phospho(ST) \\
\hline Q8NFC6 & YYSDSDDELTVEQR & 2 Phospho(ST) \\
\hline P23588 & SQSSDTEQQSPTSGGGK & Phospho(ST) \\
\hline Q08AD1 & SSSMSYVDGFIGTWPK & Phospho(Y) \\
\hline Q96EV2 & VKPASPVAQPK & Phospho(ST) \\
\hline Q96EV2 & DIKEESDEEEEDDEESGR & Phospho(ST) \\
\hline
\end{tabular}




\begin{tabular}{|c|c|c|}
\hline Protein & Peptide sequence & Variable modification \\
\hline O75821 & VTNLSEDTR & Phospho(ST) \\
\hline O14745 & EALAEAALESPRPALVR & Phospho(ST) \\
\hline Q15648 & NSSQSGGKPGSSPITK & Phospho(ST) \\
\hline Q9BPX3 & LNLAQFLNEDLS & Phospho(ST) \\
\hline P25205 & SEDESETEDEEEKSQEDQEQK & 2 Phospho(ST) \\
\hline Q641Q2 & TPSDDEEDNLFAPPK & Phospho(ST) \\
\hline Q641Q2 & TVLSLFDEEEDKMEDQNIIQAPQK & Phospho(ST) \\
\hline Q9Y4E1 & TPSDDEEDNLFAPPK & Phospho(ST) \\
\hline Q9Y4E1 & TVLSLFDEEEDKMEDQNIIQAPQK & Phospho(ST) \\
\hline P35221 & SRTSVQTEDDQLIAGQSAR & 2 Phospho(ST) \\
\hline P26232 & SRTSVQTEDDQLIAGQSAR & 2 Phospho(ST) \\
\hline Q6P158 & GLSGEEEDDEPDCCNDER & Phospho(ST) \\
\hline O60271 & SASQSSLDKLDQELK & Phospho(ST) \\
\hline Q9UKV3 & SLSPGVSR & Phospho(ST) \\
\hline Q9UKV3 & TTSPLEEEER & Phospho(ST) \\
\hline Q9UKV3 & AESPAEKVPEESVLPLVQK & Phospho(ST) \\
\hline P21291 & GFGFGQGAGALVHSE & Phospho(ST) \\
\hline \multirow[t]{2}{*}{ P46379 } & APPQTHLPSGASSGTGSASATHGGGSPPGT & 3 Phospho(ST) \\
\hline & RGPGASVHDR & \\
\hline P35269 & LDTGPQSLSGKSTPQPPSGK & 2 Phospho(ST) \\
\hline P35269 & IHDLEDDLEMSSDASDASGEEGGRVPK & 4 Phospho(ST) \\
\hline P35269 & GVDEQSDSSEESEEEKPPEEDKEEEEEK & 4 Phospho(ST) \\
\hline Q0ZGT2 & KESLSPGK & Phospho(ST) \\
\hline Q9Y2D5 & QVLQSTQSPR & Phospho(ST) \\
\hline Q9Y2D5 & TLSMIEEEIR & Phospho(ST) \\
\hline Q14432 & SLPPGLLR & Phospho(ST) \\
\hline Q16643 & MAPTPIPTRSPSDSSTASTPVAEQIER & Phospho(ST) \\
\hline Q14978 & VAGGAAPSKPASAK & Phospho(ST) \\
\hline Q15424 & ADSLLAVVK & Phospho(ST) \\
\hline Q15424 & SVVSFDKVK & Phospho(ST) \\
\hline Q15424 & SRDSESHSR & Phospho(ST) \\
\hline Q15424 & ETINNLDTSSSDFTILQEIEEPSLEPENEK & Phospho(ST) \\
\hline Q14151 & ADSLLAVVK & Phospho(ST) \\
\hline Q13442 & KSLDSDESEDEEDDYQQK & 2 Phospho(ST) \\
\hline Q96GM8 & AADSDDGAVSAPAASDGGVSK & Phospho(ST) \\
\hline Q96K21 & LPDSDDDEDEETAIQR & Phospho(ST) \\
\hline Q5JTV8 & SELGNQSPSTSSR & Phospho(ST) \\
\hline Q5JTV8 & VNFSEEGETEEDDQDSSHSSVTTVK & 2 Phospho(ST) \\
\hline Q9Y2U8 & ENYSDSEEEDDDDVASSR & Phospho(ST); Phospho(Y) \\
\hline Q12888 & SEDMPFSPK & Phospho(ST) \\
\hline Q12888 & SEDPPTTPIR & Phospho(ST) \\
\hline Q1KMD3 & SGDETPGSEVPGDK & 2 Phospho(ST) \\
\hline O43815 & FLESAAADFSDEDEDDDVDGR & Phospho(ST) \\
\hline
\end{tabular}




\begin{tabular}{|c|c|c|}
\hline Protein & Peptide sequence & Variable modification \\
\hline Q6PD62 & GGEFDEFVNDDTDDDLPISK & Phospho(ST) \\
\hline Q8WWM7 & MYPPRSPK & Phospho(ST) \\
\hline Q6PKG0 & AVTPVPTK & Phospho(ST) \\
\hline Q6PKG0 & EQEKGEGSDSKESPK & Phospho(ST) \\
\hline Q8N3X1 & RPILQLSPPGPR & Phospho(ST) \\
\hline P49327 & LFDHPESPTPNPTEPLFLAQAEVYK & Phospho(ST) \\
\hline Q9NTJ3 & REEGPPPPSPDGASSDAEPEPPSGR & Phospho(ST) \\
\hline \multirow[t]{2}{*}{ Q02241 } & YMLTHQELASDGEIETK & Oxidation(M); \\
\hline & & Phospho(ST) \\
\hline \multirow[t]{2}{*}{ Q02241 } & SNDRNSMDIQCEVDALLER & Oxidation(M); \\
\hline & & Phospho(ST) \\
\hline Q13136 & VTSSMSSPSMQPK & Phospho(ST) \\
\hline \multirow[t]{2}{*}{ Q13136 } & VTSSMSSPSMQPK & Oxidation(M); \\
\hline & & Phospho(ST) \\
\hline Q7Z460 & NSSNTSVGSPSNTIGR & Phospho(ST) \\
\hline Q9Y6X9 & KTESPIK & Phospho(ST) \\
\hline Q9Y6X9 & SPPLPAVIR & Phospho(ST) \\
\hline O60832 & AGLESGAEPGDGDSDTTK & Phospho(ST) \\
\hline O60832 & KRESESESDETPPAAPQLIK & 3 Phospho(ST) \\
\hline Q9BVJ6 & SELSQDAEPAGSQETK & Phospho(ST) \\
\hline Q9UIG0 & DHTVSGDEDYCPR & Phospho(ST) \\
\hline Q9C0C2 & VNLFPGLSPSALK & Phospho(ST) \\
\hline P52272 & MGANSLER & Phospho(ST) \\
\hline Q9H7N4 & VPSTPPPK & Phospho(ST) \\
\hline Q9H7N4 & REVLYDSEGLSGEER & 2 Phospho(ST) \\
\hline Q14684 & VAEPGAEATSSTGEESGSEHPPAVPMHNK & 2 Phospho(ST) \\
\hline Q92522 & AGGSAALSPSK & Phospho(ST) \\
\hline Q00341 & VATLNSEEESDPPTYK & Phospho(ST) \\
\hline O14639 & GVSMPNMLEPK & Phospho(ST) \\
\hline Q99733 & LDNVPHTPSSYIETLPK & Phospho(ST) \\
\hline Q12906 & LFPDTPLALDANK & Phospho(ST) \\
\hline \multirow[t]{2}{*}{ Q12906 } & DSSKGEDSAEETEAKPAVVAPAPVVEAVS & Phospho(ST) \\
\hline & TPSAAFPSDATAEQGPILTK & \\
\hline \multirow[t]{2}{*}{ Q6P2E9 } & GPGQVPTATSALSLELQEVEPLGLPQASPS & Phospho(ST) \\
\hline & $\mathrm{R}$ & \\
\hline Q9BRS2 & VVPGQFDDADSSDSENR & Phospho(ST) \\
\hline O95747 & RVPGSSGR & Phospho(ST) \\
\hline Q9NVU7 & YIEIDSDEEPR & Phospho(ST) \\
\hline Q9NVU7 & KYIEIDSDEEPR & Phospho(ST) \\
\hline Q8WUF5 & DNLTSATLPR & Phospho(ST) \\
\hline Q8WUF5 & SLGSAGPSGTLPR & Phospho(ST) \\
\hline P08559 & YHGHSMSDPGVSYR & 2 Phospho(ST) \\
\hline P29803 & YHGHSMSDPGVSYR & 2 Phospho(ST) \\
\hline
\end{tabular}




\begin{tabular}{|c|c|c|}
\hline Protein & Peptide sequence & Variable modification \\
\hline O75376 & MPIEDSSPEK & Phospho(ST) \\
\hline Q9Y6D5 & ELEKPIQSKPQSPVIQAAAVSPK & 2 Phospho(ST) \\
\hline Q9H910 & TSDIFGSPVTATSR & Phospho(ST) \\
\hline E9PAV3 & $\begin{array}{l}\text { FKVQGEAVSNIQENTQTPTVQEESEEEEVD } \\
\text { ETGVEVK }\end{array}$ & Phospho(ST) \\
\hline Q96T37 & LHSYSSPSTK & Phospho(ST) \\
\hline Q9Y383 & AMLDQLMGTSR & $\begin{array}{l}\text { Oxidation(M); } \\
\text { Phospho(ST) }\end{array}$ \\
\hline Q9Y383 & FRDQDLASCDR & Phospho(ST) \\
\hline Q9H788 & TLSSSAQEDIIR & Phospho(ST) \\
\hline Q9NYB0 & YLLGDAPVSPSSQK & Phospho(ST) \\
\hline Q8WWQ0 & VLSDSEDEEKDADVPGTSTR & 2 Phospho(ST) \\
\hline P78345 & ELLDTSFEDLSKPK & Phospho(ST) \\
\hline O60716 & SMGYDDLDYGMMSDYGTAR & Phospho(ST) \\
\hline Q13263 & $\begin{array}{l}\text { STAPSAAASASASAAASSPAGGGAEALELL } \\
\text { EHCGVCR }\end{array}$ & Phospho(ST) \\
\hline Q5QJE6 & QILIACSPVSSVR & Phospho(ST) \\
\hline Q5QJE6 & ESYTEEIVSEAESHVSGISR & Phospho(ST) \\
\hline Q9H307 & QQDSQPEEVMDVLEMVENVK & Phospho(ST) \\
\hline Q9H307 & QESDPEDDDVKKPALQSSVVATSK & Phospho(ST) \\
\hline Q9H307 & ESEKQQDSQPEEVMDVLEMVENVK & Phospho(ST) \\
\hline Q00526 & IGEGTYGVVYK & Phospho(Y) \\
\hline Q00526 & IGEGTYGVVYK & Phospho(ST) \\
\hline Q00526 & IGEGTYGVVYK & Phospho(ST); Phospho(Y) \\
\hline Q9P2B4 & DLSPTLIDNSAAK & Phospho(ST) \\
\hline Q5JSH3 & GKELSDQATASPIVAR & Phospho(ST) \\
\hline Q9NX63 & YSGAYGASVSDEELK & Phospho(ST) \\
\hline Q13459 & SVKEAGSSGR & Phospho(ST) \\
\hline Q7Z5L9 & LEEPPELNRQSPNPR & Phospho(ST) \\
\hline Q96C19 & ADLNQGIGEPQSPSR & Phospho(ST) \\
\hline Q96C19 & RADLNQGIGEPQSPSR & Phospho(ST) \\
\hline Q9UHB6 & QWTSLSLR & Phospho(ST) \\
\hline Q8NHQ9 & TVDLGISDLEDDC & Phospho(ST) \\
\hline Q8NHQ9 & EEGSDIEDEDMEELLNDTR & Phospho(ST) \\
\hline Q9BW71 & EESEESEAEPVQR & 2 Phospho(ST) \\
\hline Q9BW71 & SLKESEQESEEEILAQK & 2 Phospho(ST) \\
\hline Q9Y5T5 & NINMDNDLEVLTSSPTR & Phospho(ST) \\
\hline Q9Y5T5 & TVEDEDQDSEEEKDNDSYIK & Phospho(ST) \\
\hline Q14244 & LLTPTHSFLAR & Phospho(ST) \\
\hline Q14244 & AAPAQVRPPSPGNIRPVK & Phospho(ST) \\
\hline Q9BZZ5 & ASEDTTSGSPPKK & Phospho(ST) \\
\hline Q9UPQ0 & SRQTPSPDVVLR & 2 Phospho(ST) \\
\hline Q96B36 & SSDEENGPPSSPDLDR & Phospho(ST) \\
\hline
\end{tabular}




\begin{tabular}{|c|c|c|}
\hline Protein & Peptide sequence & Variable modification \\
\hline P17812 & DTYSDRSGSSSPDSEITELK & 2 Phospho(ST) \\
\hline Q13523 & VQSGMGLILQGYESGSEEEGEIHEK & Phospho(ST); Phospho(Y) \\
\hline Q8TDD1 & IDDRDSDEEGASDR & Phospho(ST) \\
\hline Q14157 & YPSSISSSPQK & 2 Phospho(ST) \\
\hline Q14157 & NPSDSAVHSPFTK & Phospho(ST) \\
\hline Q14157 & DGGQTESNEEGKENR & Phospho(ST) \\
\hline Q66PJ3 & SAGEEEDGPVLTDEQK & Phospho(ST) \\
\hline O95400 & KGPGQPSSPQR & Phospho(ST) \\
\hline P20810 & $\begin{array}{l}\text { KEGITGPPADSSKPIGPDDAIDALSSDFTCG } \\
\text { SPTAAGK }\end{array}$ & Phospho(ST) \\
\hline Q13185 & LTWHSCPEDEAQ & Phospho(ST) \\
\hline Q13185 & RKSLSDSESDDSK & 2 Phospho(ST) \\
\hline Q5H9L2 & GTDDSPKDSQEDLQER & Phospho(ST) \\
\hline Q15154 & INFSDLDQR & Phospho(ST) \\
\hline O94804 & QVAEQGGDLSPAANR & Phospho(ST) \\
\hline Q9H019 & ASSFADMMGILK & Phospho(ST) \\
\hline O76021 & KEQTPEHGK & Phospho(ST) \\
\hline O76021 & AAESETPGKSPEK & Phospho(ST) \\
\hline O00148 & NCPHVVVGTPGR & Phospho(ST) \\
\hline Q13620 & SATDGNTSTTPPTSAK & Phospho(ST) \\
\hline Q13620 & MAEESSSSSSSSSPTAATSQQQQLK & Phospho(ST) \\
\hline Q14195 & GMYDGPVFDLTTTPK & Phospho(ST) \\
\hline Q6KC79 & GSRPPLILQSQSLPCSSPR & Phospho(ST) \\
\hline Q92508 & TASELLLDR & Phospho(ST) \\
\hline P40855 & NATDLQNSSMSEEELTK & Phospho(ST) \\
\hline O94875 & SFTSSSPSSPSR & Phospho(ST) \\
\hline P49840 & GEPNVSYICSR & Phospho(Y) \\
\hline Q9Y4H2 & VASPTSGVK & Phospho(ST) \\
\hline Q9Y4H2 & TYSLTTPAR & Phospho(ST) \\
\hline Q9Y4H2 & TDSLAATPPAAK & Phospho(ST) \\
\hline Q9Y4H2 & SPGEYINIDFGEPGAR & Phospho(Y) \\
\hline Q9Y4H2 & SKSQSSGSSATHPISVPGAR & Phospho(ST) \\
\hline P40818 & SYSSPDITQAIQEEEKR & Phospho(ST) \\
\hline Q9NX40 & KLENSPLGEALR & Phospho(ST) \\
\hline Q4G0J3 & DIEISTEEEKDTGDLK & 2 Phospho(ST) \\
\hline Q53EL6 & DSGRGDSVSDSGSDALR & Phospho(ST) \\
\hline Q07666 & SGSMDPSGAHPSVR & $\begin{array}{l}\text { Oxidation(M); } \\
\text { Phospho(ST) }\end{array}$ \\
\hline Q9BYW2 & IISENSMDSAISDATSELEGK & Phospho(ST) \\
\hline O75448 & LLSSNEDDANILSSPTDR & Phospho(ST) \\
\hline O15042 & EKDECTPTR & Phospho(ST) \\
\hline P46087 & GADSELSTVPSVTK & 3 Phospho(ST) \\
\hline P16615 & AEIGIAMGSGTAVAKTASEMVLADDNFSTI & Oxidation(M); \\
\hline
\end{tabular}




\begin{tabular}{|c|c|c|}
\hline Protein & Peptide sequence & Variable modification \\
\hline & VAAVEEGRAIYNNMK & Phospho(ST) \\
\hline Q53T59 & GEDAEESLEEEEALDPLGIMR & Phospho(ST) \\
\hline Q9P2E9 & KVDTTPNQGK & Phospho(ST) \\
\hline \multirow[t]{2}{*}{ Q14155 } & MSGFIYQGK & Oxidation(M); \\
\hline & & Phospho(ST) \\
\hline Q14C86 & SSDIVSSVR & Phospho(ST) \\
\hline Q05682 & VTSPTKV & Phospho(ST) \\
\hline Q8N122 & VLDTSSLTQSAPASPTNK & 2 Phospho(ST) \\
\hline Q04637 & AASLTEDR & Phospho(ST) \\
\hline Q04637 & SFSKEVEER & Phospho(ST) \\
\hline Q6WCQ1 & SKSVIEQVSWDT & Phospho(ST) \\
\hline Q6WCQ1 & TKDQPDGSSLSPAQSPSQSQPPAASSLR & Phospho(ST) \\
\hline P23508 & LQSVQATGPSSPGR & Phospho(ST) \\
\hline Q9Y6M7 & SFADIGK & Phospho(ST) \\
\hline Q9Y6M7 & MLQDDDDTVHLPFEGGSLLQIPVK & Phospho(ST) \\
\hline Q5T3I0 & DLESCSDDDNQGSK & 2 Phospho(ST) \\
\hline O14979 & KDLTEYLSR & Phospho(ST) \\
\hline P51532 & KIPDPDSDDVSEVDAR & Phospho(ST) \\
\hline Q13439 & VPSVESLFR & Phospho(ST) \\
\hline Q86VM9 & RPNTSPDR & Phospho(ST) \\
\hline Q86VM9 & LGVSVSPSR & 2 Phospho(ST) \\
\hline Q9P265 & IQQLLNTLK & Phospho(ST) \\
\hline P29590 & KASPEAASTPR & Phospho(ST) \\
\hline Q9NZI8 & QGSPVAAGAPAK & Phospho(ST) \\
\hline P17535 & LASPELER & Phospho(ST) \\
\hline \multirow[t]{2}{*}{ P17535 } & LAALKDEPQTVPDVPSFGESPPLSPIDMDT & 2 Phospho(ST) \\
\hline & QER & \\
\hline P05412 & LASPELER & Phospho(ST) \\
\hline A6NKF1 & TLEEVVMAEEEDEGTDRPGSPA & Phospho(ST) \\
\hline Q9NQZ2 & TSAAACAVTDLSDDSDFDEK & 2 Phospho(ST) \\
\hline Q96ST3 & YMNSDTTSPELR & Phospho(ST) \\
\hline Q9NPQ8 & GLMAGGRPEGQYSEDEDTDTDEYKEAK & Phospho(ST); Phospho(Y) \\
\hline Q9NRY4 & TSFSVGSDDELGPIR & Phospho(ST) \\
\hline P78332 & KEESPPPPK & Phospho(ST) \\
\hline Q15758 & GPAGDATVASEK & Phospho(ST) \\
\hline Q9NS91 & NDLQDTEISPR & Phospho(ST) \\
\hline Q96KC8 & EDAEGVAAEEEQEGDSGEQETGATDARPR & Phospho(ST) \\
\hline \multirow[t]{2}{*}{ A2RU67 } & SPLGEAPEPDSDAEVAEAAKPHLSEVTTEG & Phospho(ST) \\
\hline & YPSEPLGGLEQK & \\
\hline Q13045 & NAEAVLQSPGLSGK & Phospho(ST) \\
\hline P17275 & DATPPVSPINMEDQER & Phospho(ST) \\
\hline O95155 & SQSMDIDGVSCEK & Phospho(ST) \\
\hline Q9NZ63 & RRGDSESEEDEQDSEEVR & 2 Phospho(ST) \\
\hline
\end{tabular}




\begin{tabular}{|c|c|c|}
\hline Protein & Peptide sequence & Variable modification \\
\hline Q9BXP5 & HELSPPQK & Phospho(ST) \\
\hline P02794 & HTLGDSDNES & Phospho(ST) \\
\hline O75400 & SDSPESDAER & Phospho(ST) \\
\hline Q08499 & QHEVEIPSPTQK & Phospho(ST) \\
\hline Q14669 & AQTAPTKTSPR & Phospho(ST) \\
\hline P40425 & TAVSVTQGGHSR & Phospho(ST) \\
\hline O60231 & QQTEKPESEDEWER & Phospho(ST) \\
\hline Q01664 & FIQELSGSSPK & Phospho(ST) \\
\hline P53621 & NLSPGAVESDVR & Phospho(ST) \\
\hline P41227 & GNSPPSSGEACR & Phospho(ST) \\
\hline Q96MU7 & GISPIVFDR & Phospho(ST) \\
\hline Q8NAV1 & RSPTLR & Phospho(ST) \\
\hline Q8NAV1 & VPSPDHR & Phospho(ST) \\
\hline Q8NAV1 & VSALEEDMDDVESSEEEEEEDEKLER & 2 Phospho(ST) \\
\hline P78347 & ESTSSKSPPR & Phospho(ST) \\
\hline P78347 & TNTPVKEDWNVR & Phospho(ST) \\
\hline P62753 & LSSLRASTSK & 2 Phospho(ST) \\
\hline Q5T200 & LISDSVER & Phospho(ST) \\
\hline Q5T200 & NTEESSSPVRK & Phospho(ST) \\
\hline Q5T200 & SLSPSHLTEDR & Phospho(ST) \\
\hline Q5T200 & TSAVSSPLLDQQR & Phospho(ST) \\
\hline Q9H1B7 & KASPEPPDSAEGALK & Phospho(ST) \\
\hline O94874 & KDDDSDDESQSSHTGK & Phospho(ST) \\
\hline Q9UKZ1 & MPGGGASAASGR & Phospho(ST) \\
\hline \multirow[t]{2}{*}{ Q9UKZ1 } & TPRQIALMDVGNMGQSVDISGLQLALAER & Oxidation(M); \\
\hline & QSELPTQSK & 2 Phospho(ST) \\
\hline Q99543 & NASASFQELEDK & Phospho(ST) \\
\hline Q99543 & ELSEESEDEELQLEEFPMLK & Phospho(ST) \\
\hline Q99543 & ELSEESEDEELQLEEFPMLK & 2 Phospho(ST) \\
\hline P82094 & SVSEINSDDELSGK & Phospho(ST) \\
\hline O43294 & SPKPAAPAAPPFSSSSGVLGTGLCELDR & Phospho(ST) \\
\hline Q14152 & LGDSSLSR & Phospho(ST) \\
\hline Q8IUD2 & RTNSTGGSSGSSVGGGSGK & Phospho(ST) \\
\hline Q8IY81 & DLIDNSFNR & Phospho(ST) \\
\hline Q96NB3 & EEENADSDDEGELQDLLSQDWR & Phospho(ST) \\
\hline \multirow[t]{2}{*}{ P98175 } & GLVAAYSGESDSEEEQER & 2 Phospho(ST); \\
\hline & & Phospho(Y) \\
\hline \multirow[t]{2}{*}{ Q8WUA2 } & DFIIQTGDPTGTGRGGESIFGQLYGDQASFF & 3 Phospho(ST); \\
\hline & EAEKVPR & Phospho(Y) \\
\hline Q08945 & EGMNPSYDEYADSDEDQHDAYLER & Phospho(ST) \\
\hline Q96QT4 & FFVSTPSQPSCK & Phospho(ST) \\
\hline Q8WXI9 & GRLTPSPDIIVLSDNEASSPR & 3 Phospho(ST) \\
\hline Q8WXI9 & GRLTPSPDIIVLSDNEASSPR & 4 Phospho(ST) \\
\hline
\end{tabular}




\begin{tabular}{|c|c|c|}
\hline Protein & Peptide sequence & Variable modification \\
\hline P50914 & AALLKASPK & Phospho(ST) \\
\hline P49454 & LALSPLSLGK & Phospho(ST) \\
\hline Q92597 & TASGSSVTSLDGTR & Phospho(ST) \\
\hline Q9BUR4 & EGDPVSLSTPLETEFGSPSELSPR & Phospho(ST) \\
\hline Q5VV41 & GLNTSQESDDDILDESSSPEGTQK & Phospho(ST) \\
\hline Q13177 & YLSFTPPEK & Phospho(ST) \\
\hline Q15836 & ADALQAGASQFETSAAK & Phospho(ST) \\
\hline O15027 & ADSGPTQPPLSLSPAPETK & Phospho(ST) \\
\hline Q96IZ0 & RSTGVVNIPAAECLDEYEDDEAGQK & Phospho(ST) \\
\hline P30050 & IGPLGLSPK & Phospho(ST) \\
\hline Q5JSZ5 & LLSFSPEEFPTLK & Phospho(ST) \\
\hline Q3YEC7 & RADDFPVRDDPSDVTDEDEGPAEPPPPPK & 2 Phospho(ST) \\
\hline Q3YEC7 & $\begin{array}{l}\text { AAQQDSDSDGEALGGNPMVAGFQDDVDL } \\
\text { EDQPR }\end{array}$ & 2 Phospho(ST) \\
\hline Q6ZSZ5 & SLSPILPGR & Phospho(ST) \\
\hline Q86VQ1 & GPSPSSPTPPAAAAPAEQAPR & 2 Phospho(ST) \\
\hline Q09161 & RRSDDDDR & Phospho(ST) \\
\hline O75694 & PSSLLGAAMPASTSAAALQEALENAGR & Phospho(ST) \\
\hline O43166 & LIDLESPTPESQK & Phospho(ST) \\
\hline A2RRP1 & AGEEDEGEEDSDSDYEISAK & Phospho(ST) \\
\hline P38159 & RDVYLSPR & Phospho(ST) \\
\hline P38159 & VEQATKPSFESGR & Phospho(ST) \\
\hline O15013 & SLIAQDHR & Phospho(ST) \\
\hline Q5T0N5 & TISDGTISASK & Phospho(ST) \\
\hline Q76FK4 & VSCHDSDDDIMR & Phospho(ST) \\
\hline Q86XP3 & $\begin{array}{l}\text { YMAENPTAGVVQEEEEDNLEYDSDGNPIA } \\
\text { PTK }\end{array}$ & Phospho(ST) \\
\hline P05114 & TEESPASDEAGEK & Phospho(ST) \\
\hline Q9C0E2 & HQQQLLASPGSSTVDNK & Phospho(ST) \\
\hline Q5SNT2 & RTSPSSLPGR & Phospho(ST) \\
\hline Q13435 & GFEEEHKDSDDDSSDDEQEK & 3 Phospho(ST) \\
\hline Q15527 & DLGSTEDGDGTDDFLTDKEDEK & Phospho(ST) \\
\hline Q53EU6 & NSASVGIIQR & Phospho(ST) \\
\hline Q8ND56 & SPTMEQAVQTASAHLPAPAAVGR & $\begin{array}{l}\text { Oxidation(M); } \\
\text { Phospho(ST) }\end{array}$ \\
\hline O75909 & KPSPQPSSPR & Phospho(ST) \\
\hline Q9H0G5 & $\begin{array}{l}\text { KTQQLHPVLQKPSVFGNDSDDDDETSVSES } \\
\text { LQR }\end{array}$ & Phospho(ST) \\
\hline Q96G23 & LVEDERSDREETESSEGEEAAAGGGAK & 4 Phospho(ST) \\
\hline P62070 & FQEQECPPSPEPTR & Phospho(ST) \\
\hline P18887 & AIGSTSKPQESPK & Phospho(ST) \\
\hline Q08170 & SKPNLPSESR & Phospho(ST) \\
\hline Q08170 & SKSKDQAEEK & Phospho(ST) \\
\hline
\end{tabular}




\begin{tabular}{|c|c|c|}
\hline Protein & Peptide sequence & Variable modification \\
\hline O95295 & AMLDSGIYPPGSPGK & Phospho(ST) \\
\hline Q9H8V3 & ANTPELK & Phospho(ST) \\
\hline Q13610 & LQEEGGGSDEEETGSPSEDGMQSAR & Phospho(ST) \\
\hline P0DJ93 & ELVGDTGSQEGDHEPSGSETEEDTSSSPHR & 2 Phospho(ST) \\
\hline Q8IVF2 & IKLPSFR & Phospho(ST) \\
\hline Q8IVF2 & IPSLGWSPSK & Phospho(ST) \\
\hline Q9UHF7 & AGFNYESPSK & Phospho(ST) \\
\hline Q9UHF7 & ATEETGQAQSGQANCQGLSPVSVASK & Phospho(ST) \\
\hline Q8N9T8 & YVDEENSDGETSNHR & Phospho(ST) \\
\hline O95071 & RISQSQPVR & Phospho(ST) \\
\hline Q68EM7 & $\begin{array}{l}\text { AESSSGGGTVPSSAGILEQGPSPGDGSPPKP } \\
\mathrm{K}\end{array}$ & Phospho(ST) \\
\hline O00470 & EGGSKSDSEDITR & Phospho(ST) \\
\hline Q9UPT8 & TGSGSPFAGNSPAR & 2 Phospho(ST) \\
\hline Q14573 & LGFVDVQNCISR & Phospho(ST) \\
\hline P49321 & KPEEESPR & Phospho(ST) \\
\hline P49321 & $\begin{array}{l}\text { EGEETEGSEEDDKENDKTEEMPNDSVLEN } \\
\mathrm{K}\end{array}$ & 2 Phospho(ST) \\
\hline O15541 & YGVYEDENYEVGSDDEEIPFK & Phospho(ST) \\
\hline Q9P2N5 & LQLGTPPPLLAAR & Phospho(ST) \\
\hline Q9NV56 & VLTANSNPSSPSAAK & 2 Phospho(ST) \\
\hline Q8N3V7 & DRASPAAAEEVVPEWASCLK & Phospho(ST) \\
\hline P42684 & KSEESAAPSR & Phospho(ST) \\
\hline P42684 & GAQASSGSPALPR & Phospho(ST) \\
\hline O75128 & GCLTTPNSPSMHSR & Phospho(ST) \\
\hline Q86UP2 & QPTPPSEAAASK & Phospho(ST) \\
\hline Q9UKM9 & $\begin{array}{l}\text { TRDDGDEEGLLTHSEEELEHSQDTDADDG } \\
\text { ALQ }\end{array}$ & Phospho(ST) \\
\hline Q7L2J0 & DITDPLSLNTCTDEGHVVLASPLK & Phospho(ST) \\
\hline Q7L2J0 & $\begin{array}{l}\text { DEVVSPLPSALQGPSGSLSAPPAASVISAPP } \\
\text { SSSSR }\end{array}$ & Phospho(ST) \\
\hline P36578 & ILKSPEIQR & Phospho(ST) \\
\hline Q02880 & YTFDFSEEEDDDADDDDDDNNDLEELK & Phospho(ST) \\
\hline Q9H813 & STSYQELSEELVQVVENSELADEQDKETVR & Phospho(ST) \\
\hline O43491 & EISPGSGPGEIR & Phospho(ST) \\
\hline Q8N5G2 & NASGVVNSSPR & Phospho(ST) \\
\hline O43847 & RGSLSNAGDPEIVK & Phospho(ST) \\
\hline P53985 & SKASLEK & Phospho(ST) \\
\hline Q86SQ0 & SGAASMPSSPK & Phospho(ST) \\
\hline Q00587 & SDSLLSFR & 2 Phospho(ST) \\
\hline P11908 & VTSIADR & Phospho(ST) \\
\hline Q9NYV4 & GSPVFLPR & Phospho(ST) \\
\hline Q13596 & LPPPFPGLEPESEGAAGGSEPEAGDSDTEGE & 3 Phospho(ST) \\
\hline
\end{tabular}




\begin{tabular}{|c|c|c|}
\hline Protein & Peptide sequence & Variable modification \\
\hline & DIFTGAAVVSK & \\
\hline Q03188 & SWITIPR & Phospho(ST) \\
\hline Q03188 & SEESPVYSNSSVR & Phospho(ST) \\
\hline Q7L1Q6 & NAEEESESEAEEGD & Phospho(ST) \\
\hline O00401 & VIYDFIEK & Phospho(Y) \\
\hline Q96MW1 & $\begin{array}{l}\text { AALLAQYADVTDEEDEADEKDDSGATTM } \\
\text { NIGSDK }\end{array}$ & Phospho(ST) \\
\hline Q9Y618 & SLIGSPGR & Phospho(ST) \\
\hline Q9Y618 & EGTPPPPPPSR & Phospho(ST) \\
\hline Q9Y618 & SLGYHGSSYSPEGVEPVSPVSSPSLTHDK & Phospho(ST); Phospho(Y) \\
\hline Q9BZE4 & TAAGEYDSVSESEDEEMLEIR & 3 Phospho(ST) \\
\hline Q96PE2 & LADILSPR & Phospho(ST) \\
\hline P12270 & TDGFAEAIHSPQVAGVPR & Phospho(ST) \\
\hline P12270 & GAILSEEELAAMSPTAAAVAK & Phospho(ST) \\
\hline P15924 & SMSFQGIR & Phospho(ST) \\
\hline P15924 & SSSFSDTLEESSPIAAIFDTENLEK & Phospho(ST) \\
\hline O00267 & $\begin{array}{l}\text { SAAGSEKEEEPEDEEEEEEEEEYDEEEEEED } \\
\text { DDRPPK }\end{array}$ & 2 Phospho(ST) \\
\hline Q8IZ21 & KDTLAMK & Phospho(ST) \\
\hline Q8IZ21 & SLPITIEMLK & Phospho(ST) \\
\hline P49802 & KSGNSMDR & $\begin{array}{l}\text { Oxidation(M); } \\
\text { Phospho(ST) }\end{array}$ \\
\hline Q14186 & VFIDQNLSPGK & Phospho(ST) \\
\hline Q9UPN9 & TFAPLPEFEQEEDDGEVTEDSDEDFIQPR & 2 Phospho(ST) \\
\hline Q01814 & SATSSSPGSPIHSLETSL & Phospho(ST) \\
\hline O14641 & DSSEHGAGGHR & Phospho(ST) \\
\hline Q53H80 & TLDFDPLLSPASPK & Phospho(ST) \\
\hline P78310 & TPQSPTLPPAK & Phospho(ST) \\
\hline Q9NQG6 & AISAPTSPTR & Phospho(ST) \\
\hline Q9BW61 & TDSPDMHEDT & Phospho(ST) \\
\hline Q6NZI2 & RGSSPDVHALLEITEESDAVLVDKSDSD & 4 Phospho(ST) \\
\hline Q8WY36 & TADGRVSPAGGTLDDKPK & Phospho(ST) \\
\hline Q9NYJ8 & AIGNNSATSPR & Phospho(ST) \\
\hline P27448 & GIAPASPMLGNASNPNK & Phospho(ST) \\
\hline O43303 & LPSPEPSMSPK & Phospho(ST) \\
\hline Q9UHR6 & $\begin{array}{l}\text { GAGPQLLEELDNAPGSDAAELELAPARTPP } \\
\text { DSVK }\end{array}$ & Phospho(ST) \\
\hline Q9Y5K6 & SVDFDSLTVR & Phospho(ST) \\
\hline Q9NZH5 & LFQLGPPSPVK & Phospho(ST) \\
\hline Q32MZ4 & CTLPEHESPSQDISDACEAESTER & Phospho(ST) \\
\hline Q9H7D0 & SLQLMDNR & Phospho(ST) \\
\hline Q86YR5 & APSSDEECFFDLLTK & Phospho(ST) \\
\hline Q9NSD4 & RSSGLSR & Phospho(ST) \\
\hline
\end{tabular}




\begin{tabular}{|c|c|c|}
\hline Protein & Peptide sequence & Variable modification \\
\hline Q9H6S0 & VLMKSPSPALHPPQK & Phospho(ST) \\
\hline Q9NXE8 & MANSSPVLSK & Phospho(ST) \\
\hline Q86Y82 & AGSRLSAEER & Phospho(ST) \\
\hline O60264 & TPTSPLK & Phospho(ST) \\
\hline O60264 & $\begin{array}{l}\text { GGPEGVAAQAVASAASAGPADAEMEEIFD } \\
\text { DASPGK }\end{array}$ & Phospho(ST) \\
\hline A0FGR8 & EPTPSIASDISLPIATQELR & 2 Phospho(ST) \\
\hline A0FGR8 & EPTPSIASDISLPIATQELR & 3 Phospho(ST) \\
\hline P49815 & SMSGGHGLR & $\begin{array}{l}\text { Oxidation(M); } \\
\text { Phospho(ST) }\end{array}$ \\
\hline Q96RK0 & VFSPVIR & Phospho(ST) \\
\hline Q8WUM0 & RSSLSSR & Phospho(ST) \\
\hline Q66K74 & SASPHDVDLCLVSPCEFEHR & Phospho(ST) \\
\hline P11216 & KQISVR & Phospho(ST) \\
\hline Q99459 & LVLPAPQISDAELQEVVK & Phospho(ST) \\
\hline Q99459 & GGLNTPLHESDFSGVTPQR & Phospho(ST) \\
\hline P31948 & HDSPEDVK & Phospho(ST) \\
\hline Q92615 & SPSPAHLPDDPK & Phospho(ST) \\
\hline Q92615 & $\begin{array}{l}\text { FTSSQTQSPTPPKPPSPSFELGLSSFPPLPGA } \\
\text { AGNLK }\end{array}$ & Phospho(ST) \\
\hline Q92615 & $\begin{array}{l}\text { FTSSQTQSPTPPKPPSPSFELGLSSFPPLPGA } \\
\text { AGNLK }\end{array}$ & 2 Phospho(ST) \\
\hline P46100 & DFDSSEDEKHSK & 2 Phospho(ST) \\
\hline P46100 & $\begin{array}{l}\text { ATSSSNPSSPAPDWYKDFVTDADAEVLEHS } \\
\text { GK }\end{array}$ & 2 Phospho(ST) \\
\hline P15923 & AGAPGALSPSYDGGLHGLQSK & Phospho(ST) \\
\hline Q9H6H4 & SFSMQDLR & Phospho(ST) \\
\hline Q01804 & TAADVVSPGANSVDSR & Phospho(ST) \\
\hline Q9P206 & RSTVLGLPQHVQK & Phospho(ST) \\
\hline Q8NEY8 & DNTFFRESPVGR & Phospho(ST) \\
\hline O94851 & RDSFSR & Phospho(ST) \\
\hline Q9UHB7 & TSSSSKEVK & Phospho(ST) \\
\hline Q9UHB7 & MFSPMEEK & Phospho(ST) \\
\hline Q9UHB7 & SSSPGKPQAVSSLNSSHSR & Phospho(ST) \\
\hline P83916 & ADSDSEDKGEESKPK & Phospho(ST) \\
\hline Q86XL3 & TSLPLSPVK & Phospho(ST) \\
\hline Q70E73 & HGSLSSR & Phospho(ST) \\
\hline Q9Y678 & SLEELPVDIILASVG & Phospho(ST) \\
\hline O75717 & SHILEDDENSVDISMLK & Phospho(ST) \\
\hline O75717 & $\begin{array}{l}\text { DYNDLFDGDDMSNAGDFLNDNAVEIPSFS } \\
\mathrm{K}\end{array}$ & Phospho(ST) \\
\hline O75717 & $\begin{array}{l}\text { VEKDYNDLFDGDDMSNAGDFLNDNAVEIP } \\
\text { SFSK }\end{array}$ & Phospho(ST) \\
\hline
\end{tabular}




\begin{tabular}{|c|c|c|}
\hline Protein & Peptide sequence & Variable modification \\
\hline \multirow[t]{2}{*}{ Q96QU8 } & MASEEASLR & Oxidation(M); \\
\hline & & Phospho(ST) \\
\hline O60293 & LDSSPVSSPR & Phospho(ST) \\
\hline Q96C57 & EKEESPQPR & Phospho(ST) \\
\hline Q13573 & GPPSPPAPVMHSPSR & 2 Phospho(ST) \\
\hline Q02040 & VVPEDGSPEKR & Phospho(ST) \\
\hline Q9UBQ5 & IDFDSVSSIMASSQ & Phospho(ST) \\
\hline O15357 & DASDGEDEKPPLPPR & Phospho(ST) \\
\hline \multirow{2}{*}{ P43307 } & VEMGTSSQNDVDMSWIPQETLNQINKASP & 2 Oxidation $(\mathrm{M})$ \\
\hline & $\mathrm{R}$ & Phospho(ST) \\
\hline P31629 & RDLSPR & Phospho(ST) \\
\hline P11831 & ALIQTCLNSPDSPPR & Phospho(ST) \\
\hline O15014 & ELESPLTPGK & Phospho(ST) \\
\hline Q8TAD8 & GHSHQR & Phospho(ST) \\
\hline Q8TAD8 & LSPEVAPPAHR & Phospho(ST) \\
\hline Q13733 & GHSHQR & Phospho(ST) \\
\hline P13984 & HYQGEEKSD & Phospho(ST) \\
\hline Q8N4C8 & SDSVLPASHGHLPQAGSLER & Phospho(ST) \\
\hline Q8N4C8 & GTPKPPGPPAQPPGPPNASSNPDLR & Phospho(ST) \\
\hline Q96A49 & EQDLPLAEAVRPKTPPVVIK & Phospho(ST) \\
\hline Q13242 & GRDSPYQSR & Phospho(ST) \\
\hline \multirow[t]{2}{*}{ Q9H6Y2 } & TCEERPAEDGSDEEDPDSMEAPTR & Oxidation(M); \\
\hline & & Phospho(ST) \\
\hline Q96JP5 & RSSPSARPPDVPGQQPQAAK & Phospho(ST) \\
\hline O60504 & HPSSPSALR & Phospho(ST) \\
\hline Q9BU76 & RPAEATSSPTSPERPR & Phospho(ST) \\
\hline Q8IZW8 & ASSPHGLGSPLVASPR & Phospho(ST) \\
\hline P49959 & NYSEVIEVDESDVEEDIFPTTSK & Phospho(ST) \\
\hline Q13469 & TSPDPSPVSAAPSK & Phospho(ST) \\
\hline P56192 & TSPKPAVVETVTTAKPQQIQALMDEVTK & Phospho(ST) \\
\hline Q8WVM8 & VNLEESSGVENSPAGARPK & Phospho(ST) \\
\hline Q9BVC5 & KSPSGPVKSPPLSPVGTTPVK & 2 Phospho(ST) \\
\hline Q5T1M5 & SSLSGDEEDELFK & 2 Phospho(ST) \\
\hline O15446 & ILEGPQQSLSGSPLQPIPASPPPQIPPGLRPR & Phospho(ST) \\
\hline A0MZ66 & SMPVLGSVSSVTK & Phospho(ST) \\
\hline P35251 & ESVSPEDSEK & Phospho(ST) \\
\hline P12694 & IGHHSTSDDSSAYR & Phospho(ST) \\
\hline Q86UU1 & NLAATLQDIETK & Phospho(ST) \\
\hline Q9UIF9 & YSEEGLSPSK & Phospho(ST) \\
\hline Q9HCD6 & YQQETSVSQLPGRPKSPLSK & Phospho(ST) \\
\hline Q71RC2 & EQYVPPRSPK & Phospho(ST) \\
\hline Q8TCJ2 & ENPPVEDSSDEDDKR & Phospho(ST) \\
\hline Q92614 & YSHSYLSDSDTEAK & 2 Phospho(ST) \\
\hline
\end{tabular}




\begin{tabular}{|c|c|c|c|}
\hline Protein & Peptide sequence & Variable modification & \\
\hline Q96JG6 & SAYQEYDSDSDVPEELKR & Phospho(ST) & \\
\hline Q92900 & AYQHGGVTGLSQY & Phospho(ST) & \\
\hline Q9UFC0 & ACASPSAQVEGSPVAGSDGSQPAVK & Phospho(ST) & \\
\hline Q9Y2D8 & ERVDDSTGTVISDVEEDAGELSR & Phospho(ST) & \\
\hline Q9H0H5 & LSTIDESGSILSDISFDKTDESLDWDSSLVK & 2 Phospho(ST) & \\
\hline Q5T5Y3 & APVHFVEPLSPTGVAGHR & Phospho(ST) & \\
\hline Q96S94 & GLLPGGTQVLDGTSGFSPAPK & Phospho(ST) & \\
\hline O94826 & ASPAPGSGHPEGPGAHLDMNSLDR & Phospho(ST) & \\
\hline O15164 & NESEDNKFSDDSDDDFVQPR & 2 Phospho(ST) & \\
\hline O14497 & SHHAPMSPGSSGGGGQPLAR & Phospho(ST) & \\
\hline Q9BXB5 & SSPGSVAASPSGGGGR & Phospho(ST) & \\
\hline P48634 & EGPEPPEEVPPPTTPPVPK & Phospho(ST) & \\
\hline P00533 & ELVEPLTPSGEAPNQALLR & Phospho(ST) & \\
\hline O60934 & IPNYQLSPTKLPSINK & Phospho(ST) & \\
\hline Q99549 & GYTSDDDTWEPEIHLEDCK & Phospho(ST) & \\
\hline Q6FI81 & KSSPSVKPAVDPAAAK & Phospho(ST) & \\
\hline P61289 & ITSEAEDLVANFFPK & Phospho(ST) & \\
\hline Q6ZNJ1 & RISQVSSGETEYNPTEAR & Phospho(ST) & \\
\hline Q9BXY0 & $\begin{array}{l}\text { ALEQQEAESDSSDTEEKDDDDDDEEDVGK } \\
\mathrm{R}\end{array}$ & 3 Phospho(ST) & \\
\hline Q96QT6 & VLTPPQAAGDGILATTANQR & Phospho(ST) & \\
\hline P51580 & TSLDIEEYSDTEVQK & Phospho(ST) & \\
\hline O43818 & MNEEISSDSESESLAPR & 3 Phospho(ST) & \\
\hline Q9P1Y6 & EASPAPLAQGEPGR & Phospho(ST) & \\
\hline Q86WC4 & SSTSFANIQENSN & Phospho(ST) & \\
\hline Q8N3D4 & KGSDALRPPVPQGEDEVPK & Phospho(ST) & \\
\hline Q13131 & HTLDELNPQKSK & Phospho(ST) & \\
\hline Q13131 & TSCGSPNYAAPEVISGR & Phospho(ST) & \\
\hline Q9NRG0 & REEDEENDNDNESDHDEADS & Phospho(ST) & \\
\hline Q9NUQ3 & NLVSPAYCTQESR & Phospho(ST) & \\
\hline Q92466 & SRSPLELEPEAK & Phospho(ST) & \\
\hline O43395 & IKWDEQTSNTKGDDDEESDEEAVK & Phospho(ST) & \\
\hline Q5VZK9 & SSGFISELPSEEGK & Phospho(ST) & \\
\hline Q5VZK9 & $\begin{array}{l}\text { TEVTIIINALGSNTSLTKVDISGNGMGDMG } \\
\text { AKMLAK }\end{array}$ & $\begin{array}{l}3 \quad \text { Oxidation(M); } \\
\text { Phospho(ST) }\end{array}$ & 3 \\
\hline Q99700 & RGPEVTSQGVQTSSPACK & Phospho(ST) & \\
\hline P42224 & LQTTDNLLPMSPEEFDEVSR & Phospho(ST) & \\
\hline Q14934 & FGLGSPLPSPR & 2 Phospho(ST) & \\
\hline Q96SN8 & GNLELRPGGAHPGTCSPSRPGS & Phospho(ST) & \\
\hline Q69YQ0 & TSSASRPASLPR & 2 Phospho(ST) & \\
\hline Q00610 & GILRTPDTIR & Phospho(ST) & \\
\hline O00712 & KPEKPLFSSASPQDSSPR & Phospho(ST) & \\
\hline Q09666 & GGVDVTLPR & Phospho(ST) & \\
\hline
\end{tabular}




\begin{tabular}{|c|c|c|}
\hline Protein & Peptide sequence & Variable modification \\
\hline Q09666 & GGVTGSPEASISGSKGDLK & Phospho(ST) \\
\hline Q09666 & GPAFNMASPESDFGINLK & Phospho(ST) \\
\hline P02545 & LSPSPTSQR & Phospho(ST) \\
\hline P02545 & SVGGSGGGSFGDNLVTR & Phospho(ST) \\
\hline P02545 & GRASSHSSQTQGGGSVTK & 3 Phospho(ST) \\
\hline Q9UQ35 & TRTPLR & Phospho(ST) \\
\hline Q9UQ35 & SRTSPVTR & Phospho(ST) \\
\hline Q9UQ35 & RSASSPR & 3 Phospho(ST) \\
\hline Q9UQ35 & RPSPQPSPR & 2 Phospho(ST) \\
\hline \multirow[t]{2}{*}{ Q9UQ35 } & HSLSGSSPGMK & Oxidation(M); \\
\hline & & Phospho(ST) \\
\hline Q9UQ35 & QSHSSSSPHPK & Phospho(ST) \\
\hline Q9UQ35 & AQTPPGPSLSGSK & Phospho(ST) \\
\hline Q9UQ35 & SRSRTPPVTR & 3 Phospho(ST) \\
\hline Q9UQ35 & SRTPPSAPSQSR & 2 Phospho(ST) \\
\hline Q9UQ35 & RSSRSSPELTR & 3 Phospho(ST) \\
\hline Q9UQ35 & SSGHSSSELSPDAVEK & 2 Phospho(ST) \\
\hline Q9UQ35 & SGSSQELDVKPSASPQER & Phospho(ST) \\
\hline Q9UQ35 & SGSSQELDVKPSASPQER & 2 Phospho(ST) \\
\hline Q9UQ35 & HASSSPESPKPAPAPGSHR & 3 Phospho(ST) \\
\hline Q9UQ35 & RGEGDAPFSEPGTTSTQRPSSPETATK & 2 Phospho(ST) \\
\hline P10412 & KATGAATPKK & Phospho(ST) \\
\hline P16401 & AVKKTPK & Phospho(ST) \\
\hline \multirow[t]{2}{*}{ P21333 } & CSGPGLSPGMVR & Oxidation(M); \\
\hline & & Phospho(ST) \\
\hline Q8IYB3 & SPSPAPPPR & 2 Phospho(ST) \\
\hline Q8IYB3 & RSPSPPPTR & 2 Phospho(ST) \\
\hline Q8IYB3 & KVELSESEEDKGGK & 2 Phospho(ST) \\
\hline Q8IYB3 & RVSSSRSVSGSPEPAAK & 6 Phospho(ST) \\
\hline Q15149 & SSSVGSSSSYPISPAVSR & 3 Phospho(ST) \\
\hline \multirow[t]{2}{*}{ Q8NE71 } & GGNVFAALIQDQSEEEEEEEKHPPKPAKPE & Phospho(ST) \\
\hline & $\mathrm{K}$ & \\
\hline P27824 & SDAEEDGGTVSQEEEDRKPK & Phospho(ST) \\
\hline \multirow[t]{2}{*}{ P51858 } & SCVEEPEPEPEAAEGDGDKKGNAEGSSDEE & 2 Phospho(ST) \\
\hline & GK & \\
\hline P42167 & EPTPVLGSGAAAAGR & Phospho(ST) \\
\hline P42166 & EPTPVLGSGAAAAGR & Phospho(ST) \\
\hline P07900 & EEKESEDKPEIEDVGSDEEEEK & Phospho(ST) \\
\hline \multirow[t]{2}{*}{ Q15185 } & FSEMMNNMGGDEDVDLPEVDGADDDSQD & 2 Phospho(ST) \\
\hline & SDDEKMPDLE & \\
\hline Q9NYF8 & SPEIHR & Phospho(ST) \\
\hline Q9NYF8 & QKSPEIHR & Phospho(ST) \\
\hline Q9NYF8 & AEGEPQEESPLK & Phospho(ST) \\
\hline
\end{tabular}




\begin{tabular}{|c|c|c|}
\hline Protein & Peptide sequence & Variable modification \\
\hline Q9NYF8 & YSPSQNSPIHHIPSR & Phospho(ST); Phospho(Y) \\
\hline P46821 & SLMSSPEDLTK & 2 Phospho(ST) \\
\hline P46821 & SKPLAASPKPAGLK & Phospho(ST) \\
\hline P46821 & SPSDSGYSYETIGK & Phospho(ST) \\
\hline P46821 & QGVDDIEKFEDEGAGFEESSETGDYEEK & Phospho(ST) \\
\hline P29692 & KPATPAEDDEDDDIDLFGSDNEEEDK & 2 Phospho(ST) \\
\hline P20700 & TTIPEEEEEEEEAAGVVVEEELFHQQGTPR & Phospho(ST) \\
\hline Q13428 & AALAPAKESPR & Phospho(ST) \\
\hline Q13428 & ASSVSTKESPAR & Phospho(ST) \\
\hline Q13428 & DDPDGKQEAKPQQAAGMLSPK & Phospho(ST) \\
\hline P05787 & FASFIDK & Phospho(ST) \\
\hline P08729 & FASFIDK & Phospho(ST) \\
\hline P02538 & FASFIDK & Phospho(ST) \\
\hline O95678 & FASFIDK & Phospho(ST) \\
\hline \multirow[t]{2}{*}{ Q14247 } & LPSSPVYEDAASFKAELSYRGPVSGTEPEP & Oxidation(M) \\
\hline & VYSMEAADYR & Phospho(ST); Phospho(Y) \\
\hline Q9NR30 & KEEPSQNDISPK & Phospho(ST) \\
\hline O60841 & QSFDDNDSEELEDK & Phospho(ST) \\
\hline P07910 & NDKSEEEQSSSSVK & Phospho(ST) \\
\hline \multirow[t]{2}{*}{ P07910 } & MESEGGADDSAEEGDLLDDDDNEDRGDD & 2 Phospho(ST) \\
\hline & QLELIK & \\
\hline Q12888 & TSSGTSLSAMHSSGSSGK & Phospho(ST) \\
\hline Q12888 & SNVSSPATPTASSSSSTTPTR & Phospho(ST) \\
\hline Q12888 & ETAVPGPLGIEDISPNLSPDDK & Phospho(ST) \\
\hline P19338 & VVVSPTK & Phospho(ST) \\
\hline P19338 & VVVSPTKK & Phospho(ST) \\
\hline \multirow[t]{2}{*}{ P19338 } & KEDSDEEEDDDSEEDEEDDEDEDEDEDEIE & 2 Phospho(ST) \\
\hline & PAAMK & \\
\hline \multirow[t]{2}{*}{ P19338 } & KEDSDEEEDDDSEEDEEDDEDEDEDEDEIE & Oxidation(M); \\
\hline & PAAMK & 2 Phospho(ST) \\
\hline P49792 & HSTPSPTR & Phospho(ST) \\
\hline P27816 & VDISKVSSK & Phospho(ST) \\
\hline P27816 & DGVLTLANNVTPAK & Phospho(ST) \\
\hline P27816 & DMESPTKLDVTLAK & Phospho(ST) \\
\hline Q01105 & RQSPLPPQK & Phospho(ST) \\
\hline P49736 & RTDALTSSPGR & 3 Phospho(ST) \\
\hline P49736 & AIPELDAYEAEGLALDDEDVEELTASQR & Phospho(ST) \\
\hline \multirow[t]{2}{*}{ Q86WB0 } & SMGTGDTPGLEVPSSPLRK & Oxidation(M); \\
\hline & & Phospho(ST) \\
\hline P17096 & EEEEGISQESSEEEQ & 3 Phospho(ST) \\
\hline P05783 & TVQSLEIDLDSMR & Phospho(ST) \\
\hline \multirow[t]{2}{*}{ P29966 } & EAPAEGEAAEPGSPTAAEGEAASAASSTSS & Phospho(ST) \\
\hline & PK & \\
\hline
\end{tabular}




\begin{tabular}{|c|c|c|}
\hline Protein & Peptide sequence & Variable modification \\
\hline Q9UKV3 & RLSQPESAEK & Phospho(ST) \\
\hline O00567 & EELMSSDLEETAGSTSIPK & Phospho(ST) \\
\hline Q8IY81 & GTEASSGTEAATGLEGEEK & Phospho(ST) \\
\hline Q8N9T8 & AFVEDSEDEDGAGEGGSSLLQK & Phospho(ST) \\
\hline O60271 & ERPISLGIFPLPAGDGLLTPDAQK & Phospho(ST) \\
\hline Q5JSH3 & EYVSNDAAQSDDEEK & Phospho(ST) \\
\hline B5ME19 & QNPEQSADEDAEK & Phospho(ST) \\
\hline B5ME19 & QPLLLSEDEEDTKR & Phospho(ST) \\
\hline Q02952 & RGSSSDEEGGPK & Phospho(ST) \\
\hline Q02952 & SAESPTSPVTSETGSTFK & Phospho(ST) \\
\hline Q16637 & GTGQSDDSDIWDDTALIK & Phospho(ST) \\
\hline Q5JTV8 & LQQQHSEQPPLQPSPVMTR & $\begin{array}{l}\text { Oxidation(M); } \\
\text { Phospho(ST) }\end{array}$ \\
\hline Q5JTV8 & DSHSSEEDEASSQTDLSQTISK & 2 Phospho(ST) \\
\hline Q16643 & SPSDSSTASTPVAEQIER & Phospho(ST) \\
\hline P46379 & VGDPPQPLPEEPMEVQGAERASPEPQR & Phospho(ST) \\
\hline Q00839 & $\begin{array}{l}\text { LQAALDDEEAGGRPAMEPGNGSLDLGGDS } \\
\text { AGR }\end{array}$ & Phospho(ST) \\
\hline O60716 & GSLASLDSLR & 2 Phospho(ST) \\
\hline O60716 & APSRQDVYGPQPQVR & Phospho(ST) \\
\hline P49006 & GEVPPKETPK & Phospho(ST) \\
\hline P49006 & GDVTAEEAAGASPAK & Phospho(ST) \\
\hline P18615 & SGAHSSASPPR & Phospho(ST) \\
\hline Q9Y4E1 & GLFSDEEDSEDLFSSQSASNLK & Phospho(ST) \\
\hline O95817 & AAPSTAPAEATPPKPGEAEAPPK & Phospho(ST) \\
\hline O75592 & SKSDSYTLDPDTLR & Phospho(ST) \\
\hline P22314 & ATLPSPDKLPGFK & Phospho(ST) \\
\hline Q9Y4H2 & SPGEYINIDFGEPGAR & Phospho(ST) \\
\hline Q9Y4H2 & SSEGGVGVGPGGGDEPPTSPR & Phospho(ST) \\
\hline Q9Y4H2 & HSSETFSSTTTVTPVSPSFAHNPK & 2 Phospho(ST) \\
\hline O43823 & VDSEGDFSENDDAAGDFR & 2 Phospho(ST) \\
\hline P28290 & SQSLPTTLLSPVR & Phospho(ST) \\
\hline P28290 & SADNLSCPSPLNVMEPVTELMQEQSYLK & 3 Phospho(ST) \\
\hline P25205 & DGDSYDPYDFSDTEEEMPQVHTPK & $\begin{array}{l}\text { Oxidation(M); } \\
2 \text { Phospho(ST) }\end{array}$ \\
\hline Q7Z5L9 & GPADSLSTAAGAAELSAEGAGKSR & 2 Phospho(ST) \\
\hline P05114 & TEESPASDEAGEK & 2 Phospho(ST) \\
\hline Q9Y2W1 & SPEIHR & Phospho(ST) \\
\hline Q9Y2W1 & TDSEKPFRGSQSPK & 2 Phospho(ST) \\
\hline Q8WWM7 & QGSGRESPSLASR & Phospho(ST) \\
\hline O75376 & SHVSSEPYEPISPPQVPVVHEK & Phospho(ST) \\
\hline P18669 & SYDVPPPPMEPDHPFYSNISK & Phospho(ST) \\
\hline Q14432 & RTSLPCIPR & Phospho(ST) \\
\hline
\end{tabular}




\begin{tabular}{|c|c|c|}
\hline Protein & Peptide sequence & Variable modification \\
\hline Q14432 & ISPLSSPCSSPLQGTPASSLVSK & Phospho(ST) \\
\hline P02765 & CDSSPDSAEDVRK & Phospho(ST) \\
\hline O76021 & EKSPSLGK & Phospho(ST) \\
\hline P08621 & SRDKEER & Phospho(ST) \\
\hline Q14980 & ATSCFPRPMTPR & Phospho(ST) \\
\hline Q969E4 & $\begin{array}{l}\text { NEGNLENEGKPEDEVEPDDEGKSDEEEKP } \\
\text { DVEGK }\end{array}$ & Phospho(ST) \\
\hline P60174 & VTNGAFTGEISPGMIK & Phospho(ST) \\
\hline O14929 & LISPYK & Phospho(ST) \\
\hline O43768 & YFDSGDYNMAK & $\begin{array}{l}\text { Oxidation(M); } \\
\text { Phospho(ST) }\end{array}$ \\
\hline P56211 & YFDSGDYNMAK & $\begin{array}{l}\text { Oxidation(M); } \\
\text { Phospho(ST) }\end{array}$ \\
\hline P35659 & KESESEDSSDDEPLIKK & 4 Phospho(ST) \\
\hline Q9NZT2 & KVEEEGSPGDPDHEASTQGR & Phospho(ST) \\
\hline P08559 & YHGHSMSDPGVSYR & $\begin{array}{l}\text { Oxidation(M); } \\
2 \text { Phospho(ST) }\end{array}$ \\
\hline P29803 & YHGHSMSDPGVSYR & $\begin{array}{l}\text { Oxidation(M); } \\
2 \text { Phospho(ST) }\end{array}$ \\
\hline P52292 & NVSSFPDDATSPLQENR & Phospho(ST) \\
\hline Q96JM3 & GGSPDLWK & Phospho(ST) \\
\hline Q96JM3 & AVPPVSPELR & Phospho(ST) \\
\hline Q96JM3 & KPGPSGPSESPK & Phospho(ST) \\
\hline Q13185 & SLSDSESDDSK & Phospho(ST) \\
\hline Q9Y3T9 & EAARSPDKPGGSPSASR & 2 Phospho(ST) \\
\hline Q9Y3T9 & DLFDLNSSEEDDTEGFSER & Phospho(ST) \\
\hline P46937 & QASTDAGTAGALTPQHVR & Phospho(ST) \\
\hline $\mathrm{P} 05455$ & SPSKPLPEVTDEYK & Phospho(ST) \\
\hline Q9NW82 & TMFAQVESDDEEAKNEPEWK & Phospho(ST) \\
\hline Q9C0C2 & VSGAGFSPSSK & Phospho(ST) \\
\hline Q9C0C2 & ASRVPSSDEEVVEEPQSR & 2 Phospho(ST) \\
\hline Q92597 & SHTSEGAHLDITPNSGAAGNSAGPK & Phospho(ST) \\
\hline P35613 & KPEDVLDDDDAGSAPLK & Phospho(ST) \\
\hline P35613 & RKPEDVLDDDDAGSAPLK & Phospho(ST) \\
\hline Q08AD1 & EALSPCPSTVSTK & Phospho(ST) \\
\hline P35251 & IIYDSDSESEETLQVK & 2 Phospho(ST) \\
\hline Q7Z2W4 & ASLEDAPVDDLTR & Phospho(ST) \\
\hline P46100 & LTPVSLSNSPIK & Phospho(ST) \\
\hline P46100 & EVIEIEDASPTK & Phospho(ST) \\
\hline P46100 & RPTETNPVTSNSDEECNETVK & Phospho(ST) \\
\hline Q13610 & EKLQEEGGGSDEEETGSPSEDGMQSAR & $\begin{array}{l}\text { Oxidation(M); } \\
\text { Phospho(ST) }\end{array}$ \\
\hline P52272 & GNFGGSFAGSFGGAGGHAPGVAR & Phospho(ST) \\
\hline
\end{tabular}




\begin{tabular}{|c|c|c|}
\hline Protein & Peptide sequence & Variable modification \\
\hline \multirow[t]{2}{*}{ Q9NWH9 } & MGDGRAGAGMITQHSSNASPINRIVQISGN & 2 Oxidation(M); \\
\hline & SMPR & 2 Phospho(ST) \\
\hline P45973 & TADSSSSEDEEEYVVEK & Phospho(ST) \\
\hline O94906 & GYLTDLNSMIPTHGGDINDIK & Phospho(ST) \\
\hline Q14684 & TPTSSPASSPLVAK & Phospho(ST) \\
\hline Q9H307 & EIAIVHSDAEK & Phospho(ST) \\
\hline \multirow[t]{2}{*}{ Q15154 } & NIRYESASMSSTCEPCKSR & Oxidation(M); \\
\hline & & Phospho(ST) \\
\hline \multirow[t]{2}{*}{ P08651 } & NWTEDMEGGISSPVKK & Oxidation(M); \\
\hline & & Phospho(ST) \\
\hline Q96AC1 & EVDEVDAALSDLEITLEGGK & Phospho(ST) \\
\hline Q8WVC0 & AQGSDEDKLQNSDDDEK & 2 Phospho(ST) \\
\hline O60504 & LCDDGPQLPTSPR & Phospho(ST) \\
\hline Q9BUH6 & LAAAEETAVSPR & Phospho(ST) \\
\hline P19532 & AASDPLLSSVSPAVSK & Phospho(ST) \\
\hline \multirow[t]{2}{*}{ O75643 } & EEASDDDMEGDEAVVR & Oxidation(M); \\
\hline & & Phospho(ST) \\
\hline Q04721 & AEDEALLSEEDDPIDR & Phospho(ST) \\
\hline $\mathrm{O} 43395$ & GDDDEESDEEAVK & Phospho(ST) \\
\hline Q8IZP0 & LGSQHSPGR & Phospho(ST) \\
\hline Q8IZP0 & TNPPTQKPPSPPMSGR & Phospho(ST) \\
\hline P46087 & TNKSPEAKPLPGK & Phospho(ST) \\
\hline Q32MZ4 & IDGATQSSPAEPK & Phospho(ST) \\
\hline Q8WUF5 & RGSMEQAPAVALPPTHK & Phospho(ST) \\
\hline Q13523 & VQSGMGLILQGYESGSEEEGEIHEK & 2 Phospho(ST) \\
\hline Q8WX93 & GVTPAGFPK & Phospho(ST) \\
\hline Q8WYP5 & EVSPSDVR & Phospho(ST) \\
\hline Q99567 & EDVEVAESPLR & Phospho(ST) \\
\hline O60341 & EMDESLANLSEDEYYSEEER & Phospho(ST); Phospho(Y) \\
\hline P18887 & KTPSKPPAQLSPSVPK & Phospho(ST) \\
\hline P24928 & YSPTSPTYSPTSPK & Phospho(ST); Phospho(Y) \\
\hline Q16513 & EDVSNFDDEFTSEAPILTPPREPR & Phospho(ST) \\
\hline Q8WWQ0 & KVLSDSEDEEKDADVPGTSTR & 2 Phospho(ST) \\
\hline Q86UU0 & TAMPSPGVSQNK & Phospho(ST) \\
\hline Q86UU0 & GLLSPPMGQSGLR & Phospho(ST) \\
\hline \multirow[t]{2}{*}{ Q86UU0 } & EVDPPMGPGNLNMNMNVNMNMNMNLNV & Oxidation(M); \\
\hline & QMTPQQQMLMSQK & Phospho(ST) \\
\hline \multirow[t]{2}{*}{ Q01082 } & FLQDCQELSLWINEKMLTAQDMSYDEARN & $2 \quad$ Oxidation(M); \\
\hline & LHSK & Phospho(ST) \\
\hline Q8NDT2 & TFLEGDWTSPSK & Phospho(ST) \\
\hline Q05519 & KPIETGSPK & Phospho(ST) \\
\hline \multirow[t]{2}{*}{ Q96CB8 } & TSTVISGNSSSASVSSSVTSGLTGWAAFAA & 7 Phospho(ST) \\
\hline & KTSSAGPSTAK & \\
\hline
\end{tabular}




\begin{tabular}{|c|c|c|}
\hline Protein & Peptide sequence & Variable modification \\
\hline Q86VQ1 & VPCNVEGISPELEK & Phospho(ST) \\
\hline Q99442 & KEETPGTPK & Phospho(ST) \\
\hline P30459 & $\begin{array}{l}\text { GYYNQSEAGSHTIQMMYGCDVGPDGRLL } \\
\mathrm{R}\end{array}$ & $\begin{array}{l}\text { Oxidation(M); } \\
2 \text { Phospho(ST); } \\
\text { Phospho(Y) }\end{array}$ \\
\hline Q6P0Q8 & EQDVVTGVSPLLFR & Phospho(ST) \\
\hline Q00587 & RSDSLLSFR & Phospho(ST) \\
\hline Q00587 & ASWESLDEEWR & 2 Phospho(ST) \\
\hline Q86YP4 & SSSAVDRQR & Phospho(ST) \\
\hline Q15424 & SVVSFDK & Phospho(ST) \\
\hline Q96CX2 & $\begin{array}{l}\text { EGSLGDELLPLGYSEPEQQEGASAGAPSPT } \\
\text { LELASR }\end{array}$ & Phospho(ST) \\
\hline Q9UPN4 & SNSTTQVSQPR & Phospho(ST) \\
\hline Q9ULW0 & VLPITVPKSPAFALK & Phospho(ST) \\
\hline O43294 & $\begin{array}{l}\text { VQNHLPASGPTQPPVVSSTNEGSPSPPEPTG } \\
\mathrm{K}\end{array}$ & Phospho(ST) \\
\hline Q9Y4F1 & SPDEATAADQESEDDLSASRTSLER & Phospho(ST) \\
\hline Q14692 & $\begin{array}{l}\text { LGPQNFIDETSDIENLLKEEEDYKEENNDS } \\
\mathrm{K}\end{array}$ & Phospho(ST) \\
\hline Q7KZ85 & $\begin{array}{l}\text { GFINDDDDEDEGEEDEGSDSGDSEDDVGH } \\
\mathrm{K}\end{array}$ & 3 Phospho(ST) \\
\hline Q7L9L4 & HAEATLGSGNLR & Phospho(ST) \\
\hline Q76FK4 & TCDSITPSKSSPVPVSDTQK & Phospho(ST) \\
\hline Q96T60 & TPESQPDTPPGTPLVSQDEK & Phospho(ST) \\
\hline Q9UHD8 & SFEVEEVETPNSTPPR & Phospho(ST) \\
\hline Q13263 & $\begin{array}{l}\text { LASPSGSTSSGLEVVAPEGTSAPGGGPGTL } \\
\text { DDSATICR }\end{array}$ & Phospho(ST) \\
\hline Q15366 & GVTIPYRPKPSSSPVIFAGGQDR & Phospho(ST) \\
\hline Q92522 & AGGSAALSPSKK & Phospho(ST) \\
\hline Q6XE24 & GYGFVDFDSPAAAQK & Phospho(ST) \\
\hline Q07157 & ISKPGAVSTPVK & Phospho(ST) \\
\hline Q07157 & VQIPVSRPDPEPVSDNEEDSYDEEIHDPR & Phospho(ST); Phospho(Y) \\
\hline Q96AP0 & ELILGSETPSSPR & Phospho(ST) \\
\hline Q96HC4 & EVVKPVPITSPAVSK & Phospho(ST) \\
\hline Q14839 & KMSQPGSPSPK & Phospho(ST) \\
\hline Q14839 & STAPETAIECTQAPAPASEDEK & Phospho(ST) \\
\hline Q3KQU3 & SAVTLPR & Phospho(ST) \\
\hline Q3KQU3 & RQSLPASPR & Phospho(ST) \\
\hline Q3KQU3 & SRGPTPPAMGPR & 2 Phospho(ST) \\
\hline P23528 & KSSTPEEVK & Phospho(ST) \\
\hline P23528 & ASGVAVSDGVIK & Phospho(ST) \\
\hline Q27J81 & DPTSLLGVLQAEADSTSEGLEDAVHSR & 2 Phospho(ST) \\
\hline Q9Y618 & TSVLGGGEDGIEPVSPPEGMTEPGHSR & Oxidation(M); \\
\hline
\end{tabular}




\begin{tabular}{|c|c|c|}
\hline Protein & Peptide sequence & Variable modification \\
\hline & & Phospho(ST) \\
\hline Q9BVS4 & AEVYGSENESER & Phospho(ST) \\
\hline P29375 & EIEAMHSLR & Phospho(ST) \\
\hline Q9Y5Q9 & GKLSAEENPDDSEVPSSSGINSTK & Phospho(ST) \\
\hline O43491 & GSQPPPAAESQSSLRR & Phospho(ST) \\
\hline Q7Z5K2 & RPESPSEISPIK & 2 Phospho(ST) \\
\hline Q9NRA8 & SSSPVGLAK & Phospho(ST) \\
\hline Q96T58 & LNTVASPK & Phospho(ST) \\
\hline Q96T58 & SLVHEVGKPPQDVTDDSPPSK & Phospho(ST) \\
\hline Q96T58 & ESGVVAVSPEKSESPQKEDGLSSQLK & 2 Phospho(ST) \\
\hline O60231 & LLEDSEESSEETVSR & 3 Phospho(ST) \\
\hline P30622 & TASESISNLSEAGSIK & Phospho(ST) \\
\hline Q8N8A6 & RVNDAEPGSPEAPQGK & Phospho(ST) \\
\hline Q96NY9 & TSGGDHAPDSPSGENSPAPQGR & Phospho(ST) \\
\hline P49450 & RRSPSPTPTPGPSR & 2 Phospho(ST) \\
\hline $\mathrm{O} 75122$ & KPGSAGGPK & Phospho(ST) \\
\hline P48634 & KGNSPNSEPPTPK & Phospho(ST) \\
\hline Q8NHH9 & $\begin{array}{l}\text { RTSDPSAAVNHVSSTTSLGENYEDDDLVNS } \\
\text { DEVMK }\end{array}$ & Phospho(ST) \\
\hline Q6QNY0 & VAGEAAETDSEPEPEPEPTAAPR & 2 Phospho(ST) \\
\hline Q5T1M5 & LSLTSDPEEGDPLALGPESPGEPQPPQLK & Phospho(ST) \\
\hline Q8IUD2 & TFWSPELK & Phospho(ST) \\
\hline Q99700 & GISPVVSEHR & Phospho(ST) \\
\hline Q8N684 & SSSTEPPPPVR & Phospho(ST) \\
\hline Q9HCK8 & VSPSDTTPLVSR & Phospho(ST) \\
\hline O15155 & SLSIEIGHEVK & Phospho(ST) \\
\hline O00273 & SISASKASPPGDLQNPK & Phospho(ST) \\
\hline Q9GZY8 & SMSENAVR & Phospho(ST) \\
\hline Q9ULM3 & VQSPKPITGGLGAFTK & Phospho(ST) \\
\hline O43896 & RNSLDGGGR & Phospho(ST) \\
\hline P28066 & GVNTFSPEGR & Phospho(ST) \\
\hline Q5T200 & KTSAVSSPLLDQQR & Phospho(ST) \\
\hline Q15276 & RAQSTDSLGTSGSLQSK & 2 Phospho(ST) \\
\hline Q4LE39 & LSKPPFQTNPSPEMVSK & Phospho(ST) \\
\hline Q9Y383 & SRSHQR & Phospho(ST) \\
\hline Q86UU1 & VLTTSPSR & Phospho(ST) \\
\hline Q9ULT8 & VSTLAGPSSDDENEEESKPEKEDEPQEDAK & Phospho(ST) \\
\hline $\mathrm{P} 17480$ & GKLPESPK & Phospho(ST) \\
\hline O15446 & QEQINTEPLEDTVLSPTK & Phospho(ST) \\
\hline Q5T8P6 & LNHSPPQSSSR & Phospho(ST) \\
\hline P12036 & SPAEVKSPEK & Phospho(ST) \\
\hline Q9Y519 & SHSLSGAR & Phospho(ST) \\
\hline Q9Y519 & TLLLSSDDEF & 2 Phospho(ST) \\
\hline
\end{tabular}




\begin{tabular}{|c|c|c|}
\hline Protein & Peptide sequence & Variable modification \\
\hline Q8IWS0 & TAHNSEADLEESFNEHELEPSSPK & Phospho(ST) \\
\hline Q12830 & SPILEEK & Phospho(ST) \\
\hline Q96A49 & $\begin{array}{l}\text { TQEDEEEISTSPGVSEFVSDAFDACNLNQE } \\
\text { DLR }\end{array}$ & Phospho(ST) \\
\hline Q63ZY6 & ASAPERTPSPAPK & 2 Phospho(ST) \\
\hline Q13427 & KSPPKADEK & Phospho(ST) \\
\hline Q13427 & KENSESEKR & Phospho(ST) \\
\hline Q9BVG9 & DAGGPRPESPVPAGR & Phospho(ST) \\
\hline Q9Y3E7 & $\begin{array}{l}\text { VTDALPEPEPPGAMAASEDEEEEEEALEA } \\
\text { MQSR }\end{array}$ & Phospho(ST) \\
\hline Q14004 & ILELTPEPDRPR & Phospho(ST) \\
\hline Q14004 & LYSSEESRPYTNK & Phospho(ST) \\
\hline Q9Y446 & LSSGFDDIDLPSAVK & Phospho(ST) \\
\hline Q6FHJ7 & SSSPIPR & Phospho(ST) \\
\hline Q9BY44 & SDKSPDLAPTPAPQSTPR & Phospho(ST) \\
\hline P45974 & SAADSISESVPVGPK & Phospho(ST) \\
\hline Q9UPQ3 & FVLMATPNLSR & Phospho(ST) \\
\hline Q5BKZ1 & ISLSKSPTKTDPK & Phospho(ST) \\
\hline Q8TD55 & EGGKPPTPPPK & Phospho(ST) \\
\hline P49768 & AAVQELSSSILAGEDPEER & Phospho(ST) \\
\hline Q8TD19 & VASEAPLEHKPQVEASSPR & Phospho(ST) \\
\hline P51531 & AKPVVSDFDSDEEQDER & 2 Phospho(ST) \\
\hline P51531 & ISPIQKPQGLDPVEILQER & Phospho(ST) \\
\hline Q9H2U2 & SLVESVSSSPNK & Phospho(ST) \\
\hline Q9BUQ8 & KSSPSKDR & Phospho(ST) \\
\hline P18206 & SLLDASEEAIK & Phospho(ST) \\
\hline Q8WXI9 & GRLTPSPDIIVLSDNEASSPR & 2 Phospho(ST) \\
\hline Q6PJT7 & DLVQPDKPASPK & Phospho(ST) \\
\hline Q9UBK8 & SSRGQEEISGALPVASPASSR & Phospho(ST) \\
\hline Q96AY4 & REPESPPASAPIPLFGADTIGQR & Phospho(ST) \\
\hline Q9C0I1 & QLSLPLTQSK & Phospho(ST) \\
\hline Q66K74 & ASRPLSAR & Phospho(ST) \\
\hline Q9UGU0 & LKMSPGR & Phospho(ST) \\
\hline Q96Q15 & NLATSADTPPSTVPGTGK & 2 Phospho(ST) \\
\hline O94875 & SFTSSSPSSPSR & 2 Phospho(ST) \\
\hline Q8IXT5 & SHSKSPR & Phospho(ST) \\
\hline Q17R98 & TTTSPFFSEDTFR & Phospho(ST) \\
\hline Q05193 & RSPTSSPTPQR & 2 Phospho(ST) \\
\hline O95343 & $\begin{array}{l}\text { SILLASSGGGNGAGGGGGAGGGSGGGNGA } \\
\text { GGGGAGGAGGGGGGGSR }\end{array}$ & 2 Phospho(ST) \\
\hline Q7L4E1 & TLMLPLTEGSLR & Phospho(ST) \\
\hline Q8NG31 & SNCIMDVLEDESVQKPKFPK & Phospho(ST) \\
\hline Q8NG31 & TPSSCSSSLDSIKADGTSLDFSTYRSSQMES & 3 Phospho(ST) \\
\hline
\end{tabular}




\begin{tabular}{|c|c|c|}
\hline Protein & Peptide sequence & Variable modification \\
\hline & QFLR & \\
\hline Q8IVF2 & MPSFGVSAPGK & Phospho(ST) \\
\hline Q8IZH2 & EAQSSQATPVQTSQPDSSNIVKVSPR & Phospho(ST) \\
\hline O95677 & NNPSPPPDSDLER & Phospho(ST) \\
\hline Q8TF39 & LDESALDK & Phospho(ST) \\
\hline O60292 & DLRSPR & Phospho(ST) \\
\hline Q5SSJ5 & TVNSTRETPPK & Phospho(ST) \\
\hline Q9NXE4 & ASIQECILPDSPLYHNK & Phospho(ST) \\
\hline Q9Y2K7 & RYEDDGISDDEIEGK & Phospho(ST) \\
\hline Q07955 & VDGPRSPSYGR & 2 Phospho(ST) \\
\hline Q07955 & VKVDGPRSPSYGR & Phospho(ST); Phospho(Y) \\
\hline Q00613 & GHTDTEGRPPSPPPTSTPEK & Phospho(ST) \\
\hline Q8N7B1 & KVSEPVK & Phospho(ST) \\
\hline O75533 & TMIISPER & Phospho(ST) \\
\hline O75533 & IWDPTPSHTPAGAATPGR & Phospho(ST) \\
\hline O75533 & LSSWDQAETPGHTPSLR & 2 Phospho(ST) \\
\hline Q9ULL5 & SRPALSPLGDIDFCPPNPGPDGPR & Phospho(ST) \\
\hline P42566 & LNDPFQPFPGNDSPK & Phospho(ST) \\
\hline Q9UEY8 & TPSFLKK & Phospho(ST) \\
\hline Q9UEY8 & IEEVLSPEGSPSKSPSK & Phospho(ST) \\
\hline Q13416 & VVSAPVGKETPSK & Phospho(ST) \\
\hline P49321 & SLQENEEEEIGNLELAWDMLDLAK & Phospho(ST) \\
\hline Q13177 & IISIFSGTEK & Phospho(ST) \\
\hline O75351 & EGQPSPADEKGNDSDGEGESDDPEKK & Phospho(ST) \\
\hline \multirow[t]{2}{*}{ Q96S82 } & DMPGGFLFEGLSDDEDDFHPNTR & Oxidation(M); \\
\hline & & Phospho(ST) \\
\hline Q13769 & ALFKPPEDSQDDESDSDAEEEQTTK & 2 Phospho(ST) \\
\hline O95466 & EAAAQEAGADTPGKGEPPAPKSPPK & Phospho(ST) \\
\hline P49023 & SSPGGQDEGGFMAQGK & Phospho(ST) \\
\hline O60934 & IPNYQLSPTK & Phospho(ST) \\
\hline Q9HB58 & DNSPEPNDPEEPQEVSSTPSDKK & Phospho(ST) \\
\hline P54727 & QEKPAEKPAETPVATSPTATDSTSGDSSR & Phospho(ST) \\
\hline Q5VWN6 & SRSPLLVTVVESDPRPQGQPR & Phospho(ST) \\
\hline Q9GZR2 & APSSPVAKPGPVK & Phospho(ST) \\
\hline Q96AT1 & IQPQPPDEDGDHSDKEDEQPQVVVLK & Phospho(ST) \\
\hline Q96H55 & SLIEPVNQSIVVSGESGAGKTWTSR & 2 Phospho(ST) \\
\hline Q13153 & YMSFTDK & Phospho(ST) \\
\hline Q96IZ7 & SHSYDR & Phospho(ST) \\
\hline Q16181 & ILEQQNSSRTLEK & Phospho(ST) \\
\hline Q15061 & LQAKESPQR & Phospho(ST) \\
\hline Q9BU76 & RPAEATSSPTSPERPR & 2 Phospho(ST) \\
\hline Q9UHB7 & NSYNNSQAPSPGLGSK & Phospho(ST) \\
\hline Q9UHI6 & SYLEGSSDNQLK & Phospho(ST) \\
\hline
\end{tabular}




\begin{tabular}{lll}
\hline Protein & Peptide sequence & Variable modification \\
\hline P0C1Z6 & LLPYPTLASPASD & 2 Phospho(ST) \\
Q9NVD7 & SPTPKSPPSR & Phospho(ST) \\
P55198 & TSRHSSGGGGGGAGGGGGSMGGGGSGFIS & Oxidation(M); \\
& GR & 2 Phospho(ST) \\
Q9H330 & GESAPTLSTSPSPSSPSPTSPSPTLGR & Phospho(ST) \\
Q8NCF5 & TEFLDLDNSPLSPPSPR & Phospho(ST) \\
Q8IXQ4 & KQDDSPPRPIIGPALPPGFIK & Phospho(ST) \\
Q6V1X1 & AETTPSGGK & Phospho(ST) \\
O75151 & DSDYVYPSLESDEDNPIFK & Phospho(ST) \\
Q12857 & SVEDEMDSPGEEPFYTGQGR & Phospho(ST) \\
O14523 & EAGLSQSHDDLSNATATPSVR & Phospho(ST) \\
Q5VZ89 & RSSLPLDHGSPAQENPESEK & Phospho(ST) \\
Q9UHR4 & TPASTPVSGTPQASPMIER & 2 Phospho(ST) \\
\hline
\end{tabular}

